\title{
On the AWGN MAC With Imperfect Feedback
}

\author{
Amos Lapidoth, Fellow, IEEE, and Michèle Wigger, Member, IEEE
}

\begin{abstract}
New achievable rate regions are derived for the twouser additive white Gaussian multiple-access channel with noisy feedback. The regions exhibit the following two properties. Irrespective of the (finite) Gaussian feedback-noise variances, the regions include rate points that lie outside the no-feedback capacity region, and when the feedback-noise variances tend to zero the regions converge to the perfect-feedback capacity region.

The new achievable regions also apply to the partial-feedback setting where one of the transmitters has a noisy feedback link and the other transmitter has no feedback at all. Again, irrespective of the (finite) noise variance on the feedback link, the regions include rate points that lie outside the no-feedback capacity region. Moreover, in the case of perfect partial feedback, i.e., where the only feedback link is noise-free, for certain channel parameters the new regions include rate points that lie outside the Cover-Leung region. This answers in the negative the question posed by van der Meulen as to whether the Cover-Leung region equals the capacity region of the Gaussian multiple-access channel with perfect partial feedback.

Finally, we propose new achievable regions also for a setting where the receiver is cognizant of the realizations of the noise sequences on the feedback links.
\end{abstract}

Index Terms-Capacity, concatenated codes, Gaussian noise, linear feedback schemes, multiple-access channel, noisy feedback, partial feedback.

\section{INTRODUCTION}

$\mathbf{I}$ $\mathrm{N}$ [5], Gaarder and Wolf showed that perfect feedback from the receiver to the transmitters increases the capacity of some memoryless multiple-access channels (MACs). That this also holds for the two-user additive white Gaussian noise (AWGN) MAC was shown by Ozarow in [13], where he also determined the capacity region of this channel with perfect feedback. Here, we study the capacity region of the two-user AWGN MAC when the feedback is imperfect. We consider the following settings:

- noisy feedback, where the feedback links are corrupted by AWGN;

- noisy partialfeedback, where one of the two transmitters has a noisy feedback link whereas the other transmitter has no feedback at all;

Manuscript received January 21, 2009; revised April 12, 2010. Date of current version October 20,2010. This work was supported in part by the Swiss National Science Foundation under Grant 200021-111863/1. Parts of the material in this paper were presented at the Kailath Colloquium on Feedback Communication 2006, Stanford University, June 2006; and at the Israel IEEE Convention for Electrical Engineers 2006, Eilat, Israel, November 2006.

A. Lapidoth is with the Department of Information Technology and Electrical Engineering, ETH Zurich, Zurich, Switzerland (e-mail: lapidoth@isi.ee.ethz. ch).

M. Wigger was with the Department of Information Technology and Electrical Engineering, ETH Zurich, Zurich, Switzerland. She is now with the Communications and Electronics Department, Telecom ParisTech, Paris, France (e-mail: michele.wigger@telecom-paristech.fr).

Communicated by E. Erkip, Associate Editor for Shannon Theory.

Digital Object Identifier 10.1109/TIT.2010.2069131
- perfect partial feedback, where one of the two transmitters has a perfect (noise-free) feedback link whereas the other transmitter has no feedback at all; and

- noisy feedback with receiver side-information, where both transmitters have noisy feedback links and the receiver (but not the transmitters) is cognizant of the feedback-noise sequences.

The last setting arises, for example, when the receiver actively feeds back a quantized version of the channel output over perfect feedback links, and the feedback noises model the quantization noises, which are known to the receiver. (The MAC with quantized feedback has also been considered in [16] but under the assumption of a rate limitation on the feedback links and for the discrete memoryless case.) We show that in all these settings the capacity region is strictly larger than the no-feedback capacity region. Moreover, we show that for noisy feedback the capacity region tends to Ozarow's perfect-feedback capacity region [13] as the feedback-noise variances tend to zero. Finally, in the case of perfect partial feedback we show that for certain channel parameters the capacity region strictly contains the Cover-Leung region [4], a region that was originally derived for the perfect-feedback setting and that was later shown by Carleial [2] and (for the discrete memoryless case) by Willems and van der Meulen [21] to be achievable also in the perfect partial-feedback setting. This answers in the negative the question posed by van der Meulen in [18] as to whether the Cover-Leung region equals the capacity region of the AWGN MAC with perfect partial feedback.

To derive these results we propose coding schemes for the described settings and analyze the rates that they achieve. The idea behind our schemes is to generalize Ozarow's capacity-achieving perfect-feedback scheme to imperfect feedback. Ozarow's scheme is based on the following strategy. The transmitters first map their messages onto message points in the interval $\left[-\frac{1}{2}, \frac{1}{2}\right]$. They then successively refine the receiver's estimates of these message points by sending scaled versions of the receiver's linear minimum mean-squared errors (LMMSE) of the message points. Besides achieving capacity, Ozarow's scheme has the advantage of a double-exponential decay of the probability of error. However, a drawback of the scheme is that it is extremely sensitive to noise on the feedback links: it does not achieve any positive rate if the feedback links are not noise-free [9]. To overcome this weakness, we propose to apply an outer code around a modified version of Ozarow's scheme where the transmitters-rather than refining the message points-successively refine the input symbols from the outer code. We further modify Ozarow's scheme by allowing the transmitters to refine the input symbols by sending arbitrary linear updates (i.e., not necessarily LMMSE-updates) and by allowing the number of refinements of each input symbol to be a constant, which can be optimized and which does not grow with the blocklength. These modifications yield a scheme 
which achieves high rates also for channels with imperfect feedback. In particular, for noisy feedback and for noisy partial feedback our scheme exhibits the following key properties:

- for all finite feedback-noise variances, our scheme achieves rate points that lie outside the capacity region without feedback, and

for noisy feedback

- the scheme achieves rate regions that converge to Ozarow's perfect-feedback capacity region when the feedback-noise variances tend to zero.

Previous achievable regions for the AWGN MAC with imperfect feedback were given by Carleial [2], by Willems et al. [23] ${ }^{1}$, and by Gastpar [6]. Carleial [2] and Willems et al. [23] generalized the Cover-Leung coding scheme [4]. Gastpar's result is also based on Ozarow's scheme and on the idea of modifying it to use only a finite number of refinements which does not grow with the blocklength. ${ }^{2}$ All these regions collapse to the no-feedback capacity region when the feedback-noise variances exceed a certain threshold. Moreover, as the feedback-noise variances tend to zero the regions in [2] and [23] converge to the Cover-Leung region, which is a strict subset of Ozarow's region [1]. ${ }^{3}$

Kramer studied the discrete memoryless MAC with imperfect feedback, and presented a coding scheme for this setup that is based on code trees [10], [12].

Outer bounds on the capacity region of the AWGN MAC with noisy feedback were derived by Gastpar and Kramer [7] and Tandon and Ulukus [17] based on the idea of dependence-balance [8]. These outer bounds do not in general coincide with any known achievable regions.

The rest of the paper is outlined as follows. This section is concluded with remarks on notation. Section II describes the channel models in more detail; Section III discusses some previous achievability results. Section IV describes our results and the new coding schemes for the setting with noisy feedback. Section V shows the setting with noisy or perfect partial feedback. Section VI shows the setting with noisy feedback where the receiver has side-information. Finally, Section VII summarizes the paper.

In the following, $A^{\ell}$ denotes the $\ell$-dimensional column vector $\left(A_{1}, \ldots, A_{\ell}\right)^{\mathrm{T}} ; \operatorname{diag}\left(a_{1}, \ldots, a_{\ell}\right)$ denotes the diagonal matrix with diagonal entries $a_{1}, \ldots, a_{\ell} ; \mathrm{I}_{\ell}$ denotes the $\ell \times \ell$ identity matrix; $A^{\top}$ denotes the transpose of a matrix $A,|A|$ its determinant, and $\operatorname{tr}(A)$ its trace. Also, for zero-mean random vectors $\mathbf{S}$ and $\mathbf{T}$ we define the covariance matrices $\mathrm{K}_{\mathbf{S}, \mathbf{T}} \triangleq \mathrm{E}\left[\mathbf{S T}^{\top}\right]$ and $\mathrm{K}_{\mathrm{S}} \triangleq \mathrm{E}\left[\mathbf{S S}^{\top}\right]$. For a two-dimensional rate region $\mathcal{R}$ we denote by $\operatorname{cl}(\mathcal{R})$ its closure and by $\mathcal{R}$ its interior.

\section{Channel Model}

This paper focuses on the AWGN MAC with two transmitters that wish to transmit messages $M_{1}$ and $M_{2}$ to a single receiver.

\footnotetext{
${ }^{1}$ The result in [23] is for the discrete memoryless case, but it easily extends to the Gaussian case.

${ }^{2}$ The idea of using a finite number of refinements was already mentioned in [15]. However, only in combination with zero rate or nonvanishing probability of error.

3It can be shown that the achievable rate region in [6] converges to Ozarow's region when the feedback-noise variances tend to zero.
}

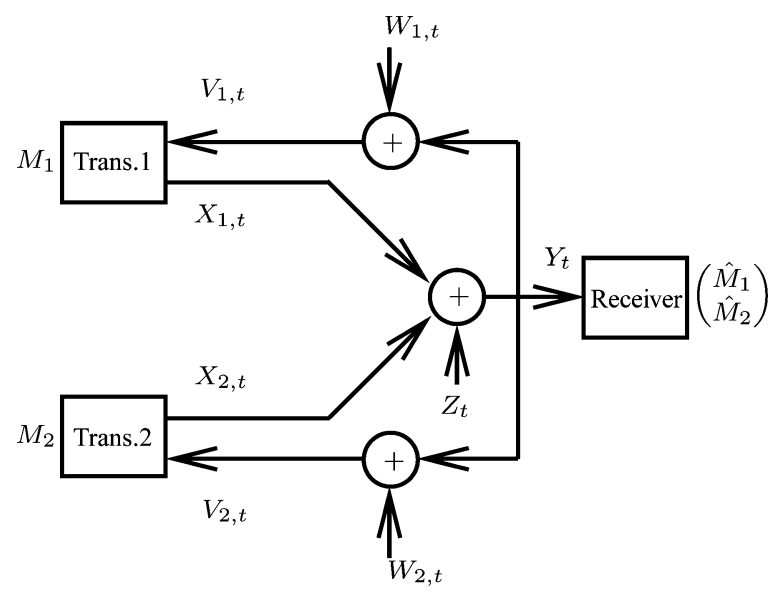

Fig. 1. AWGN MAC with noisy feedback.

The two messages are assumed to be independent and uniformly distributed over the discrete finite sets $\mathcal{M}_{1}$ and $\mathcal{M}_{2}$.

To describe the channel model (see Fig. 1), we introduce the sequence $\left\{Z_{t}\right\}$ of independent and identically distributed (IID) zero-mean variance- $N$ Gaussian random variables that will be used to model the additive noise at the receiver. Using this sequence we can describe the time- $t$ channel output $Y_{t}$ corresponding to the time- $t$ channel inputs $x_{1, t}$ and $x_{2, t}$ by

$$
Y_{t}=x_{1, t}+x_{2, t}+Z_{t} .
$$

The sequence $\left\{Z_{t}\right\}$ is assumed to be independent of the messages $\left(M_{1}, M_{2}\right)$. Also, we introduce the IID sequence of bivariate zero-mean Gaussians $\left\{\left(W_{1, t}, W_{2, t}\right)\right\}$ of covariance matrix

$$
\begin{aligned}
\mathrm{K}_{W_{1} W_{2}} & \triangleq\left(\begin{array}{cc}
\mathrm{E}\left[W_{1, t}^{2}\right] & \mathrm{E}\left[W_{1, t} W_{2, t}\right] \\
\mathrm{E}\left[W_{1, t} W_{2, t}\right] & \mathrm{E}\left[W_{2, t}^{2}\right]
\end{array}\right) \\
& =\left(\begin{array}{cc}
\sigma_{1}^{2} & \sigma_{1} \sigma_{2} \varrho \\
\sigma_{1} \sigma_{2} \varrho & \sigma_{2}^{2},
\end{array}\right)
\end{aligned}
$$

where $\sigma_{1}, \sigma_{2} \geq 0$ and $\varrho \in[-1,1]$. The sequence $\left\{\left(W_{1, t}, W_{2, t}\right)\right\}$ is used to model the additive noise corrupting the feedback links. The time- $t$ feedback output $V_{\nu, t}$ at Transmitter $\nu$ can then be modeled as

$$
V_{\nu, t}=Y_{t}+W_{\nu, t}, \quad \nu \in\{1,2\} .
$$

The sequence $\left\{\left(W_{1, t}, W_{2, t}\right)\right\}$ is assumed to be independent of $\left(M_{1}, M_{2},\left\{Z_{t}\right\}\right)$.

The transmitters observe the feedback outputs in a causal fashion, i.e., they compute their time- $t$ channel inputs $X_{1, t}$ and $X_{2, t}$ after observing all prior feedback outputs $V_{1,1}, \ldots, V_{1, t-1}$ and $V_{2,1}, \ldots, V_{2, t-1}$. Thus, for $\nu \in\{1,2\}$, Transmitter $\nu$ computes its channel inputs by mapping the Message $M_{\nu}$ and the previous feedback outputs $V_{\nu, 1}, \ldots, V_{\nu, t-1}$ into the time- $t$ channel input $X_{\nu, t}$

$$
X_{\nu, t}=\varphi_{\nu, t}^{(n)}\left(M_{\nu}, V_{\nu, 1}, \ldots, V_{\nu, t-1}\right), \quad t \in\{1, \ldots, n\}
$$

for some sequences of encoding functions

$$
\varphi_{\nu, t}^{(n)}: \mathcal{M}_{\nu} \times \mathbb{R}^{t-1} \rightarrow \mathbb{R}, \quad t \in\{1, \ldots, n\}
$$


where $n$ denotes the blocklength of the scheme. We only allow encoding functions that satisfy the power constraints

$$
\frac{1}{n} \sum_{t=1}^{n} \mathrm{E}\left[\left(\varphi_{\nu, t}^{(n)}\left(M_{\nu}, V_{\nu, 1}, \ldots, V_{\nu, t-1}\right)\right)^{2}\right] \leq P_{\nu}
$$

where the expectation is over the messages and the realizations of the channel, i.e., the noise sequences $\left\{Z_{t}\right\},\left\{W_{1, t}\right\}$, and $\left\{W_{2, t}\right\} .4$

A blocklength-n powers- $\left(P_{1}, P_{2}\right)$ feedback-code of rate pair $\left(\frac{1}{n} \log \left(\left|\mathcal{M}_{1}\right|\right), \frac{1}{n} \log \left(\left|\mathcal{M}_{2}\right|\right)\right)$ is a triple

$$
\left(\left\{\varphi_{1, t}^{(n)}\right\}_{t=1}^{n},\left\{\varphi_{2, t}^{(n)}\right\}_{t=1}^{n}, \phi^{(n)}\right)
$$

where

$$
\phi^{(n)}: \mathbb{R}^{n} \rightarrow \mathcal{M}_{1} \times \mathcal{M}_{2}
$$

and where $\left\{\varphi_{1, t}^{(n)}\right\}$ and $\left\{\varphi_{2, t}^{(n)}\right\}$ are of the form (3) and satisfy (4). In the following we say that a rate pair $\left(R_{1}, R_{2}\right)$ is achievable if for every $\delta>0$ and every sufficiently large $n$ there exists a blocklength- $n$ powers- $\left(P_{1}, P_{2}\right)$ feedback code of rates exceeding $R_{1}-\delta$ and $R_{2}-\delta$ such that the average probability of a decoding error

$$
\operatorname{Pr}\left[\phi^{(n)}\left(Y_{1}, \ldots, Y_{n}\right) \neq\left(M_{1}, M_{2}\right)\right]
$$

tends to zero as the blocklength $n \rightarrow \infty$. The set of all achievable rate pairs for this setting is called the capacity region and is denoted $\mathrm{C}_{\text {NoisyFB }}\left(P_{1}, P_{2}, N, \mathrm{~K}_{W_{1} W_{2}}\right)$.

The case $\sigma_{1}^{2}=\sigma_{2}^{2}=0$ corresponds to the special case when the feedback links are noise-free. We refer to this setting as the "perfect-feedback" setting and denote the capacity region by $\mathrm{C}_{\text {PerfectFB }}\left(P_{1}, P_{2}, N\right)$; i.e.

$$
\mathrm{C}_{\text {PerfectFB }}\left(P_{1}, P_{2}, N\right) \triangleq \mathrm{C}_{\text {NoisyFB }}\left(P_{1}, P_{2}, N, 0\right)
$$

where 0 is the $2 \times 2$ all-zero matrix.

In addition to the noisy-feedback setting we also consider the "partial-feedback" setting (see Fig. 2) where only one of the two transmitters has feedback. We assume that the transmitter with feedback is Transmitter 2. For the partial-feedback setting (2) and (3) are modified by requiring that the sequence $\left\{X_{1,1}, \ldots, X_{1, n}\right\}$ be a function of Message $M_{1}$ only. Since the sole feedback link can be noisy we shall refer to this setting also as "noisy partial feedback" and denote its capacity region by $\mathrm{C}_{\text {NoisyPartialFB }}\left(P_{1}, P_{2}, N, \sigma_{2}^{2}\right)$, where $\sigma_{2}^{2} \geq 0$ denotes the noise variance on the feedback link to Transmitter 2. In the special case of $\sigma_{2}^{2}=0$, i.e., when the sole feedback link is noise-free, we refer to the setting as "perfect partial feedback" (see Fig. 3) and denote the capacity region by $\mathrm{C}_{\text {PerfectPartialFB }}\left(P_{1}, P_{2}, N\right)$.

By the "no-feedback" setting we refer to the classical MAC where neither transmitter has a feedback link. In this case, (2) and (3) have to be modified so both sequences $\left\{X_{1,1}, \ldots, X_{1, n}\right\}$ and $\left\{X_{2,1}, \ldots, X_{2, n}\right\}$ are functions of the respective messages only. We denote the capacity region of this MAC by $\mathrm{C}_{\mathrm{NoFB}}\left(P_{1}, P_{2}, N\right)$.

\footnotetext{
${ }^{4}$ The achievability results in this paper remain valid also when the expected average block-power constraints (4) are replaced by average block-power constraints that hold with probability 1 .
}

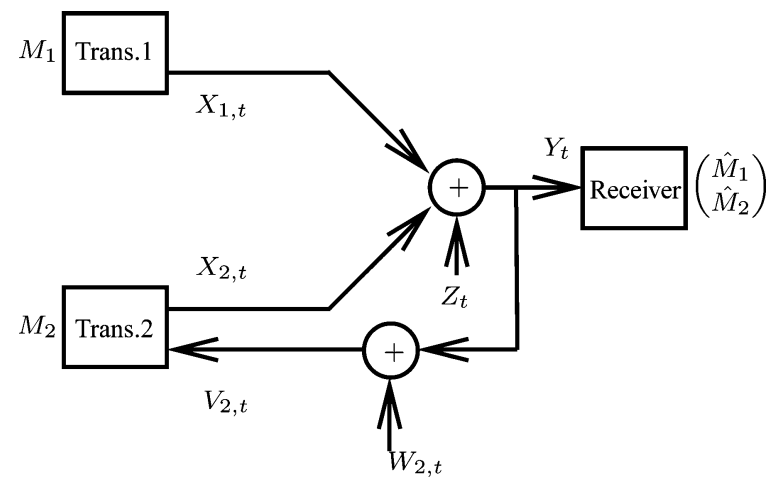

Fig. 2. AWGN MAC with noisy partial feedback.

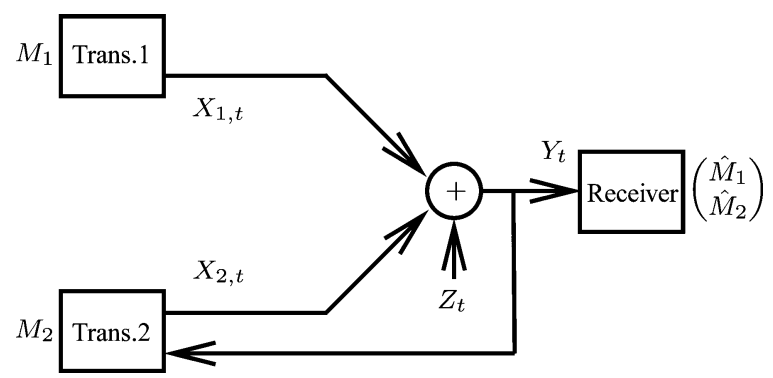

Fig. 3. AWGN MAC with perfect partial feedback.

Finally, we also consider a noisy-feedback setting where the receiver perfectly knows the realizations of the Gaussian noise sequences $\left\{W_{1, t}\right\}$ and $\left\{W_{2, t}\right\}$ corrupting the feedback signals (see also Fig. 4). ${ }^{5}$ We refer to this setting as the "noisy feedback with receiver side-information" setting. For this setting the formal description of the communication scenario is the same as in the noisy-feedback setting, except for the decoder $\phi_{\mathrm{SI}}^{(n)}$ which is of the form

$$
\begin{aligned}
\phi_{\mathrm{SI}}^{(n)}: \quad & \mathbb{R}^{n} \times \mathbb{R}^{n} \times \mathbb{R}^{n} \longrightarrow \mathcal{M}_{1} \times \mathcal{M}_{2}, \\
& \left(Y_{1}^{n}, W_{1}^{n}, W_{2}^{n}\right) \longmapsto\left(\hat{M}_{1}, \hat{M}_{2}\right) .
\end{aligned}
$$

We denote the capacity region of the MAC with noisy feedback and perfect receiver side-information by $\mathrm{C}_{\text {NoisyFBSI }}\left(P_{1}, P_{2}, N, \mathrm{~K}_{W_{1} W_{2}}\right)$.

\section{Previous Results}

We survey some previous results that are needed in the sequel.

The capacity region of the classical AWGN MAC without feedback $\mathrm{C}_{\mathrm{NoFB}}\left(P_{1}, P_{2}, N\right)$ was independently determined by Cover [3] and Wyner [25] and is given by the set of all rate pairs $\left(R_{1}, R_{2}\right)$ satisfying

$$
\begin{aligned}
R_{1} & \leq \frac{1}{2} \log \left(1+\frac{P_{1}}{N}\right), \\
R_{2} & \leq \frac{1}{2} \log \left(1+\frac{P_{2}}{N}\right), \\
R_{1}+R_{2} & \leq \frac{1}{2} \log \left(1+\frac{P_{1}+P_{2}}{N}\right) .
\end{aligned}
$$

${ }^{5}$ Since we do not consider any delay constraints and the receiver cannot actively feed back a signal, it does not matter whether the receiver learns the feedback-noise sequences $\left\{W_{1, t}\right\}$ and $\left\{W_{2, t}\right\}$ causally or acausally. 


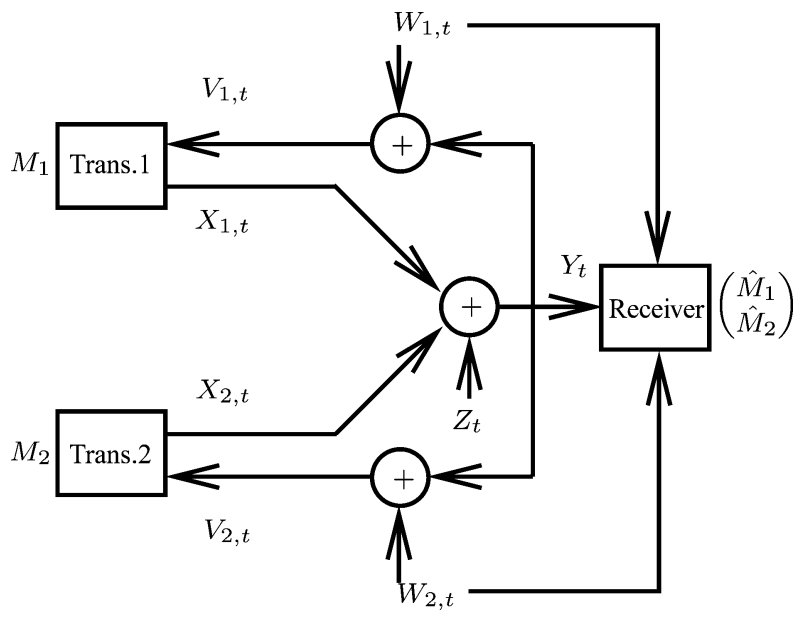

Fig. 4. AWGN MAC with noisy feedback and receiver side-information.

The capacity region of the AWGN MAC with perfect feedback $\mathrm{C}_{\text {PerfectFB }}\left(P_{1}, P_{2}, N\right)$ was determined by Ozarow [13]:

$$
\mathrm{C}_{\text {PerfectFB }}\left(P_{1}, P_{2}, N\right)=\bigcup_{\rho \in[0,1]} \mathcal{R}_{\mathrm{Oz}}^{\rho}\left(P_{1}, P_{2}, N\right)
$$

where $\mathcal{R}_{\mathrm{Oz}}^{\rho}\left(P_{1}, P_{2}, N\right)$ is the set of all rate pairs $\left(R_{1}, R_{2}\right)$ satisfying

$$
\begin{aligned}
R_{1} & \leq \frac{1}{2} \log \left(1+\frac{P_{1}\left(1-\rho^{2}\right)}{N}\right), \\
R_{2} & \leq \frac{1}{2} \log \left(1+\frac{P_{2}\left(1-\rho^{2}\right)}{N}\right), \\
R_{1}+R_{2} & \leq \frac{1}{2} \log \left(1+\frac{P_{1}+P_{2}+2 \sqrt{P_{1} P_{2}} \rho}{N}\right) .
\end{aligned}
$$

We next describe some properties of the regions $\mathcal{R}_{\mathrm{Oz}}^{\rho}\left(P_{1}, P_{2}, N\right)$ and $\mathrm{C}_{\mathrm{PerfectFB}}\left(P_{1}, P_{2}, N\right)$ that will be needed in subsequent sections. Some of the properties, Remarks III.2-III.4 and Remark III.8, were reported in [13].

Definition III.1: The parameter $\rho^{*}\left(P_{1}, P_{2}, N\right)$ (for short $\rho^{*}$ ) is defined as the unique solution in the interval $[0,1]$ of the following quartic equation in $\rho$

$$
\begin{aligned}
N\left(N+P_{1}+P_{2}\right. & \left.+2 \sqrt{P_{1} P_{2}} \rho\right) \\
= & \left(N+P_{1}\left(1-\rho^{2}\right)\right)\left(N+P_{2}\left(1-\rho^{2}\right)\right) .
\end{aligned}
$$

Remark III.2: Equation (8) is equivalent to the right-hand side (RHS) of (7c) being equal to the sum of the RHSs of (7a) and $(7 b)$.

That (8) has a unique solution in the interval $[0,1]$ can be seen as follows. At $\rho=0$ the left-hand side (LHS) of (8) is smaller than its RHS, whereas for $\rho=1$ the LHS is larger. Since the expressions on both sides of (8) are continuous, by the Intermediate Value Theorem there must exist at least one solution to $(8)$ in $[0,1]$. The uniqueness of the solution follows by noting that the LHS of (8) is strictly increasing in $\rho$ whereas the RHS is strictly decreasing in $\rho \in[0,1]$.

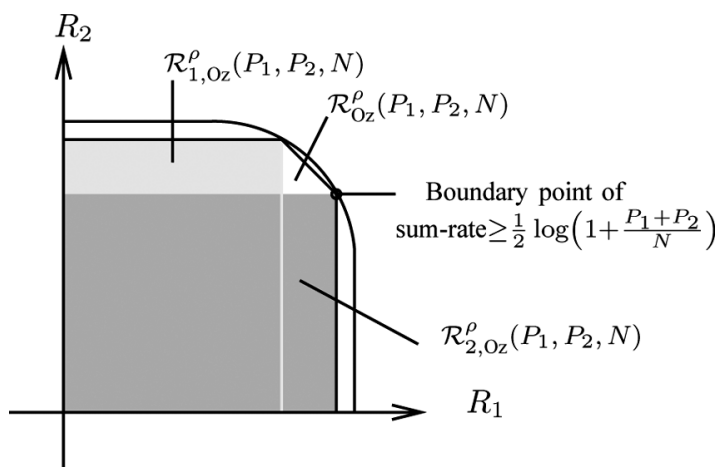

Fig. 5. Perfect-feedback capacity region with an example of $\mathcal{R}_{\mathrm{Oz}}^{\rho}, \mathcal{R}_{1, \mathrm{Oz}}^{\rho}$, and $\mathcal{R}_{2, \text { Oz }}^{\rho}$ for $0<\rho<\rho^{*}$.

Next, we discuss the region $\mathcal{R}_{\mathrm{Oz}}^{\rho}\left(P_{1}, P_{2}, N\right)$ and examine the rate constraints (7) defining the region. The RHS of single-rate constraint (7a) and the RHS of (7b) are both strictly decreasing in $\rho \in[0,1]$, whereas the RHS of the sum-rate constraint (7c) is strictly increasing in $\rho$. By these properties, by Definition III.1, and by Remark III.2 we have the following.

Remark III.3: For $\rho=\rho^{*}$ the sum of the RHSs of the singlerate constraints (7a) and (7b) equals the RHS of the sum-rate constraint (7c); for $\rho \in\left[0, \rho^{*}\right)$ the sum of the RHSs of (7a) and (7b) is strictly larger than the RHS of (7c); and for $\rho \in\left(\rho^{*}, 1\right]$ the sum of the RHSs of (7a) and (7b) is strictly smaller than the RHS of (7c).

Remark III.4: For every $\rho \in\left[0, \rho^{*}\right)$ the rate region $\mathcal{R}_{\mathrm{Oz}}^{\rho}\left(P_{1}, P_{2}, N\right)$ has the shape of a pentagon and for every $\rho \in\left[\rho^{*}, 1\right]$ the rate region $\mathcal{R}_{\mathrm{Oz}}^{\rho}\left(P_{1}, P_{2}, N\right)$ has the shape of a rectangle. Furthermore, all rectangles $\mathcal{R}_{\mathrm{Oz}}^{\rho}\left(P_{1}, P_{2}, N\right)$ for $\rho \in\left(\rho^{*}, 1\right]$ are strictly contained in the rectangle $\mathcal{R}_{\mathrm{Oz}}^{\rho^{*}}\left(P_{1}, P_{2}, N\right)$, and thus in (6) it is enough to take the union over all $\rho \in\left[0, \rho^{*}\right]$.

For the next two observations we introduce the notation of a dominant corner point as in [14]. A corner point of a given rate region is called dominant if it is of maximum sum-rate in the considered region.

Remark III.5: To every boundary point of $\mathrm{C}_{\text {PerfectrB }}\left(P_{1}, P_{2}, N\right)$ that has sum-rate larger or equal to $\frac{1}{2} \log \left(1+\frac{P_{1}+P_{2}}{N}\right)$ there exists a $\rho \in\left[0, \rho^{*}\right]$ such that this point is a dominant corner point of the region $\mathcal{R}_{\mathrm{Oz}}^{\rho}\left(P_{1}, P_{2}, N\right)$ (see Fig. 5).

Remark III. 5 follows by Remark III.4, by continuity considerations, and by the monotonicities of the constraints (7a)-(7c), see Remark III.3. To state the next observation we define the following.

Definition III.6: For each $\rho \in\left[0, \rho^{*}\right]$, we define $\mathcal{R}_{1, \mathrm{Oz}}^{\rho}\left(P_{1}, P_{2}, N\right)$ as the set of all rate pairs $\left(R_{1}, R_{2}\right)$ satisfying

$$
\begin{aligned}
R_{1} & \leq \frac{1}{2} \log \left(1+\frac{P_{1}\left(1-\rho^{2}\right)}{N}\right) \\
R_{2} & \leq \frac{1}{2} \log \left(\frac{P_{1}+P_{2}+2 \sqrt{P_{1} P_{2}} \rho+N}{P_{1}\left(1-\rho^{2}\right)+N}\right) .
\end{aligned}
$$


Similarly, $\mathcal{R}_{2, \mathrm{Oz}}^{\rho}\left(P_{1}, P_{2}, N\right)$ as the set of all rate pairs $\left(R_{1}, R_{2}\right)$ satisfying

$$
\begin{aligned}
R_{1} & \leq \frac{1}{2} \log \left(\frac{P_{1}+P_{2}+2 \sqrt{P_{1} P_{2}} \rho+N}{P_{2}\left(1-\rho^{2}\right)+N}\right), \\
R_{2} & \leq \frac{1}{2} \log \left(1+\frac{P_{2}\left(1-\rho^{2}\right)}{N}\right) .
\end{aligned}
$$

Notice that by Remark III.4, $\mathcal{R}_{1, \mathrm{Oz}}^{\rho^{*}}\left(P_{1}, P_{2}, N\right)=$ $\mathcal{R}_{2, \mathrm{Oz}}^{\rho^{*}}\left(P_{1}, P_{2}, N\right)=\mathcal{R}_{\mathrm{Oz}}^{\rho^{*}}\left(P_{1}, P_{2}, N\right)$. Also, for every $\rho \in\left[0, \rho^{*}\right]$ the regions $\mathcal{R}_{1, \mathrm{Oz}}^{\rho}\left(P_{1}, P_{2}, N\right)$ and $\mathcal{R}_{2, \mathrm{Oz}}^{\rho}\left(P_{1}, P_{2}, N\right)$ are rectangles with dominant corner point equal to one of the dominant corner points of $\mathcal{R}_{\mathrm{Oz}}^{\rho}\left(P_{1}, P_{2}, N\right)$ (see Fig. 5). By these observations and by Remark III.5 we obtain the following.

Remark III.7: The perfect-feedback capacity region can be expressed as

$$
\begin{aligned}
& \mathrm{C}_{\text {PerfectFB }}\left(P_{1}, P_{2}, N\right) \\
& \quad=\bigcup_{\rho \in\left[0, \rho^{*}\right]}\left(\mathcal{R}_{1, \mathrm{Oz}}^{\rho}\left(P_{1}, P_{2}, N\right) \cup \mathcal{R}_{2, \mathrm{Oz}}^{\rho}\left(P_{1}, P_{2}, N\right)\right) .
\end{aligned}
$$

The final remark follows from Remark III.5 and from the strict monotonicity in $\rho$ of the RHS of the sum-rate constraint (7c).

Remark III.8: The dominant corner point of the rectangle $\mathcal{R}_{\mathrm{Oz}}^{\rho^{*}}\left(P_{1}, P_{2}, N\right)$ is the only rate point of maximum sum-rate in $\mathrm{C}_{\text {PerfectFB }}\left(P_{1}, P_{2}, N\right)$.

Next, we present an achievability result for general discrete memoryless MACs and AWGN MACs with perfect feedback due to Cover and Leung [4]. The scheme is known to achieve capacity for a specific class of discrete memoryless MACs with perfect feedback [20]. However, for general channels it can be suboptimal, e.g., for Gaussian channels. For Gaussian channels the optimization problem defining the Cover-Leung region is solved by jointly Gaussian inputs, see [1], [19], and therefore the Cover-Leung region is given by

$$
\mathcal{R}_{\mathrm{CL}}\left(P_{1}, P_{2}, N\right)=\bigcup_{\rho_{1}, \rho_{2} \in[0,1]} \mathcal{R}_{\mathrm{CL}}^{\left(\rho_{1}, \rho_{2}\right)}\left(P_{1}, P_{2}, N\right)
$$

where $\mathcal{R}_{\mathrm{CL}}^{\left(\rho_{1}, \rho_{2}\right)}\left(P_{1}, P_{2}, N\right)$ comprises all rate pairs $\left(R_{1}, R_{2}\right)$ satisfying

$$
\begin{aligned}
R_{1} & \leq \frac{1}{2} \log \left(1+\frac{P_{1}\left(1-\rho_{1}^{2}\right)}{N}\right), \\
R_{2} & \leq \frac{1}{2} \log \left(1+\frac{P_{2}\left(1-\rho_{2}^{2}\right)}{N}\right), \\
R_{1}+R_{2} & \leq \frac{1}{2} \log \left(1+\frac{P_{1}+P_{2}+2 \sqrt{P_{1} P_{2}} \rho_{1} \rho_{2}}{N}\right) .
\end{aligned}
$$

Carleial [2] and Willems [21] independently proved that to achieve the Cover-Leung region $\mathcal{R}_{\mathrm{CL}}\left(P_{1}, P_{2}, N\right)$ it suffices that only one of the two transmitters have a perfect feedback link, i.e., they proved that the Cover-Leung region is achievable also in a perfect partial-feedback setting. Thereupon, van der
Meulen in a survey paper on multiple-access channels with feedback [18] posed the question whether the Cover-Leung region equals the capacity region for discrete memoryless MACs or AWGN MACs with perfect partial feedback. We will answer this question in the negative for Gaussian channels by proving that for certain channel parameters $\left(P_{1}, P_{2}, N\right)$ there exist rate pairs that lie outside the Cover-Leung region $\mathcal{R}_{\mathrm{CL}}\left(P_{1}, P_{2}, N\right)$ but that are achievable in the perfect partial-feedback setting.

For the AWGN MAC with perfect partial feedback Willems, van der Meulen, and Schalkwijk proposed a coding scheme [22] which is based on the scheme by Schalkwijk and Kailath [15]. Unfortunately, the achievable rate region can only be stated in an implicit form and is difficult to evaluate analytically and to compare to the Cover-Leung region.

In [2] Carleial proposed a coding scheme for the discrete memoryless MAC and the AWGN MAC with "generalized" feedback. In the Gaussian case, "generalized" feedback includes as special cases noisy feedback, noisy partial feedback, and perfect partial feedback. We present Carleial's region for the AWGN MAC with noisy feedback in Appendix A, where we also prove that if the feedback noise variances $\sigma_{1}^{2}$ and $\sigma_{2}^{2}$ exceed a certain threshold depending on the channel parameters $P_{1}, P_{2}$, and $N$, then Carleial's region collapses to the no-feedback capacity region in (5) (Proposition A.3 in Appendix A). For perfect partial feedback and for perfect feedback Carleial's scheme equals the Cover-Leung region $\mathcal{R}_{\mathrm{CL}}\left(P_{1}, P_{2}, N\right)$. Hence, in the case of perfect feedback Carleial's scheme is known to be strictly suboptimal for the two-user AWGN MAC.

Another coding scheme for the MAC with imperfect feedback was proposed by Willems et al. in [23]. Although proposed for discrete memoryless channels, the modifications to treat the Gaussian case are straightforward, and we state their achievable rate region for the AWGN MAC with noisy feedback in Appendix B. Like Carleial's scheme, Willems et al.'s scheme collapses to the no-feedback capacity region when the feedback-noise variances $\sigma_{1}^{2}$ and $\sigma_{2}^{2}$ exceed a certain threshold (Proposition B.3 in Appendix B), and for perfect feedback or perfect partial feedback the region equals the Cover-Leung region. Thus, for very noisy feedback, for perfect feedback, and for perfect partial feedback Carleial's region and Willems et al.'s region coincide.

\section{NOISY FEEDBACK}

In this section we focus on the setup with noisy feedback. For this setup we present new achievable regions and, based on these new regions, we derive new qualitative properties of the capacity region (Section IV-A). We also present the coding schemes corresponding to our new achievable regions (Sections IV-B-IV-D).

\section{A. Results}

In this section we present our results for noisy feedback. We begin with some definitions. For given positive integer $\eta ; \eta$-dimensional column-vectors $\mathbf{a}_{1}, \mathbf{a}_{2}$; and $\eta \times \eta$-matrices $\mathrm{B}_{1}, \mathrm{~B}_{2}$, we define the $\eta \times 2$ matrix:

$$
A_{\mathrm{r}} \triangleq\left(\begin{array}{ll}
\mathbf{a}_{1} & \mathbf{a}_{2}
\end{array}\right)
$$


the $2 \eta \times 2$ matrix

$$
A_{d} \triangleq\left(\begin{array}{cc}
a_{1} & 0 \\
0 & a_{2}
\end{array}\right)
$$

the $\eta \times 2 \eta$ matrix

$$
\mathrm{B}_{\mathrm{r}} \triangleq\left(\begin{array}{ll}
\mathrm{B}_{1} & \mathrm{~B}_{2}
\end{array}\right)
$$

the $2 \eta \times \eta$ matrix

$$
\mathrm{B}_{\mathrm{c}} \triangleq\left(\begin{array}{l}
\mathrm{B}_{1} \\
\mathrm{~B}_{2}
\end{array}\right)
$$

the $2 \eta \times 2 \eta$ block-diagonal matrix

$$
\mathrm{B}_{\mathrm{d}} \triangleq\left(\begin{array}{cc}
\mathrm{B}_{1} & 0 \\
0 & \mathrm{~B}_{2}
\end{array}\right)
$$

and the $2 \eta \times 2 \eta$ matrix

$$
\mathrm{B}_{\mathrm{b}} \triangleq\left(\begin{array}{ll}
\mathrm{B}_{1} & \mathrm{~B}_{1} \\
\mathrm{~B}_{2} & \mathrm{~B}_{2}
\end{array}\right) .
$$

Our first achievable region for noisy feedback is obtained by evaluating the rates that are achieved by the concatenated scheme in Section IV-C1. (An alternative formulation of this achievable region is presented in Section D-A of Appendix D.)

Definition IV.1: Let $\eta$ be a positive integer, let $\mathbf{a}_{1}, \mathbf{a}_{2}$ be $\eta$-dimensional vectors, let $\mathrm{B}_{1}, \mathrm{~B}_{2}$ be $\eta \times \eta$ strictly lower-triangular matrices, and let $C$ be a $2 \times \eta$ matrix. Depending on the matrix $\mathrm{C}$ the rate region $\mathcal{R}\left(N, \mathrm{~K}_{W_{1} W_{2}} ; \eta, \mathbf{a}_{1}, \mathbf{a}_{2}, \mathrm{~B}_{1}, \mathrm{~B}_{2}, \mathrm{C}\right)$ is defined as follows.

- If the product $\mathrm{CC}^{\top}$ is nonsingular, ${ }^{\top}$ then $\mathcal{R}\left(N, \mathrm{~K}_{W_{1} W_{2}} ; \eta, \mathbf{a}_{1}, \mathbf{a}_{2}, \mathrm{~B}_{1}, \mathrm{~B}_{2}, \mathrm{C}\right)$ is defined as the set of all rate-pairs $\left(R_{1}, R_{2}\right)$ satisfying the three rate constraints (18), at the bottom of the page, where $A_{r}$ and $B_{r}$ are defined in (11) and (13) and where $\otimes$ denotes the Kronecker product.

- If the product $C C^{\top}$ is singular but $C \neq 0$, then $\mathcal{R}\left(N, \mathrm{~K}_{W_{1} W_{2}} ; \eta, \mathbf{a}_{1}, \mathbf{a}_{2}, \mathrm{~B}_{1}, \mathrm{~B}_{2}, \mathrm{C}\right)$ is defined as the

${ }^{6}$ Whenever $\eta \in \mathbb{N}$ is larger than 1 , there is no loss in optimality in restricting attention to matrices $C$ so that $C^{\top}{ }^{\top}$ is nonsingular. However, for completeness, we consider all possible choices of the matrix $C$. set of all rate pairs $\left(R_{1}, R_{2}\right)$ satisfying (18) when the $2 \times \eta$ matrix $C$ is replaced by the $\eta$-dimensional row-vector obtained by choosing one of the nonzero rows of $\mathrm{C} .^{7}$

- If $\mathrm{C}=0$, then $\mathcal{R}\left(N, \mathrm{~K}_{W_{1} W_{2}} ; \eta, \mathbf{a}_{1}, \mathbf{a}_{2}, \mathrm{~B}_{1}, \mathrm{~B}_{2}, \mathrm{C}\right)$ is defined as the set containing only the origin.

Definition IV.2: Define the rate region $\mathcal{R}\left(P_{1}, P_{2}, N, \mathrm{~K}_{W_{1} W_{2}}\right)$ (or for short $\mathcal{R}$ ) as

$$
\begin{aligned}
& \mathcal{R}\left(P_{1}, P_{2}, N, \mathrm{~K}_{W_{1} W_{2}}\right) \\
& \triangleq \operatorname{cl}\left(\bigcup_{\eta, \mathbf{a}_{1}, \mathbf{a}_{2}, \mathrm{~B}_{1}, \mathrm{~B}_{2}, \mathrm{C}} \mathcal{R}\left(N, \mathrm{~K}_{W_{1} W_{2}} ; \eta, \mathbf{a}_{1}, \mathbf{a}_{2}, \mathrm{~B}_{1}, \mathrm{~B}_{2}, \mathrm{C}\right)\right)
\end{aligned}
$$

where the union is over all tuples $\left(\eta, \mathbf{a}_{1}, \mathbf{a}_{2}, \mathrm{~B}_{1}, \mathrm{~B}_{2}, \mathrm{C}\right)$ satisfying the trace constraints (19), at the bottom of the page and where the matrices $A_{d}, B_{c}, B_{d}$, and $B_{b}$ are defined in (12), (14)-(16). ${ }^{8}$

Theorem IV.3 (Noisy Feedback): The capacity region $\mathrm{C}_{\text {NoisyFB }}\left(P_{1}, P_{2}, N, \mathrm{~K}_{W_{1} W_{2}}\right)$ of the two-user AWGN MAC with noisy feedback contains the rate region $\mathcal{R}\left(P_{1}, P_{2}, N, \mathrm{~K}_{W_{1} W_{2}}\right)$, i.e.,

$$
\mathrm{C}_{\text {NoisyFB }}\left(P_{1}, P_{2}, N, \mathrm{~K}_{W_{1} W_{2}}\right) \supseteq \mathcal{R}\left(P_{1}, P_{2}, N, \mathrm{~K}_{W_{1} W_{2}}\right) .
$$

Proof: The proof is based on the concatenated scheme in Section IV-C1. As will be described ahead, for each choice of parameters $\eta, \mathbf{a}_{1}, \mathbf{a}_{2}, \mathrm{~B}_{1}, \mathrm{~B}_{2}, \mathrm{C}$ our concatenated scheme achieves the capacity region of the AWGN MAC $\xi_{1}, \xi_{2} \mapsto\left(\hat{\Xi}_{1}, \hat{\Xi}_{2}\right)$ in (37) scaled by a factor $\eta^{-1}$, i.e., it achieves the region $\mathcal{R}\left(N, \mathrm{~K}_{W_{1} W_{2}} ; \eta, \mathbf{a}_{1}, \mathbf{a}_{2}, \mathrm{~B}_{1}, \mathrm{~B}_{2}, \mathrm{C}\right)$. The details of the proof are omitted.

Remark IV.4: Evaluating the achievable region $\mathcal{R}\left(P_{1}, P_{2}, N, \mathrm{~K}_{W_{1} W_{2}}\right)$ seems to be difficult even numerically. More easily computable (but possibly smaller) achievable regions are obtained by taking the union on the RHS of

${ }^{7}$ When $C C^{\top}$ is singular then the two rows of $C$ are linearly dependent and it does not matter which nonzero row is chosen.

${ }^{8}$ Since $B_{1}$ and $B_{2}$ are strictly lower-triangular, the matrix $\left(I_{2 \eta}-B_{b}\right)$ is nonsingular and its inverse exists.

$$
\begin{aligned}
& R_{1} \leq \frac{1}{2 \eta} \log \frac{\left|\mathrm{C}\left(\mathbf{a}_{1} \mathbf{a}_{1}^{\top}+N \mathrm{I}_{\eta}+\mathrm{B}_{\mathrm{r}}\left(\mathrm{K}_{W_{1} W_{2}} \otimes \mathrm{I}_{\eta}\right) \mathrm{B}_{\mathrm{r}}^{\top}\right) \mathrm{C}^{\top}\right|}{\left|\mathrm{C}\left(N \mathrm{I}_{\eta}+\mathrm{B}_{\mathrm{r}}\left(\mathrm{K}_{W_{1} W_{2}} \otimes \mathrm{I}_{\eta}\right) \mathrm{B}_{\mathrm{r}}^{\top}\right) \mathrm{C}^{\top}\right|} \\
& R_{2} \leq \frac{1}{2 \eta} \log \frac{\left|\mathrm{C}\left(\mathbf{a}_{2} \mathbf{a}_{2}^{\top}+N \mathrm{I}_{\eta}+\mathrm{B}_{\mathrm{r}}\left(\mathrm{K}_{W_{1} W_{2}} \otimes \mathrm{I}_{\eta}\right) \mathrm{B}_{\mathrm{r}}^{\top}\right) \mathrm{C}^{\top}\right|}{\left|\mathrm{C}\left(N \mathrm{I}_{\eta}+\mathrm{B}_{\mathrm{r}}\left(\mathrm{K}_{W_{1} W_{2}} \otimes \mathrm{I}_{\eta}\right) \mathrm{B}_{\mathrm{r}}^{\top}\right) \mathrm{C}^{\top}\right|} \\
& R_{1}+R_{2} \leq \frac{1}{2 \eta} \log \frac{\left|\mathrm{C}\left(\mathrm{A}_{\mathrm{r}} \mathrm{A}_{\mathrm{r}}^{\top}+N \mathrm{I}_{\eta}+\mathrm{B}_{\mathrm{r}}\left(\mathrm{K}_{W_{1} W_{2}} \otimes \mathrm{I}_{\eta}\right) \mathrm{B}_{\mathrm{r}}^{\top}\right) \mathrm{C}^{\top}\right|}{\left|\mathrm{C}\left(N \mathrm{I}_{\eta}+\mathrm{B}_{\mathrm{r}}\left(\mathrm{K}_{W_{1} W_{2}} \otimes \mathrm{I}_{\eta}\right) \mathrm{B}_{\mathrm{r}}^{\top}\right) \mathrm{C}^{\top}\right|} \\
& \operatorname{tr}\left(\left(\begin{array}{ll}
\mathrm{I}_{\eta} & 0
\end{array}\right)\left(\mathrm{I}_{2 \eta}-\mathrm{B}_{\mathrm{b}}\right)^{-1}\left(\mathrm{~A}_{\mathrm{d}} \mathrm{A}_{\mathrm{d}}^{\top}+N \mathrm{~B}_{\mathrm{c}} \mathrm{B}_{\mathrm{c}}^{\top}+\mathrm{B}_{\mathrm{d}}\left(\mathrm{K}_{W_{1} W_{2}} \otimes \mathrm{I}_{\eta}\right) \mathrm{B}_{\mathrm{d}}^{\mathrm{T}}\right)\left(\mathrm{I}_{2 \eta}-\mathrm{B}_{\mathrm{b}}\right)^{-\mathrm{T}}\left(\begin{array}{c}
\mathrm{I}_{\eta} \\
0
\end{array}\right)\right) \leq \eta P_{1} \\
& \operatorname{tr}\left(\left(\begin{array}{ll}
0 & \mathrm{I}_{\eta}
\end{array}\right)\left(\mathrm{I}_{2 \eta}-\mathrm{B}_{\mathrm{b}}\right)^{-1}\left(\mathrm{~A}_{\mathrm{d}} \mathrm{A}_{\mathrm{d}}^{\top}+N \mathrm{~B}_{\mathrm{c}} \mathrm{B}_{\mathrm{c}}^{\top}+\mathrm{B}_{\mathrm{d}}\left(\mathrm{K}_{W_{1} W_{2}} \otimes \mathrm{I}_{\eta}\right) \mathrm{B}_{\mathrm{d}}^{\top}\right)\left(\mathrm{I}_{2 \eta}-\mathrm{B}_{\mathrm{b}}\right)^{-\mathrm{T}}\left(\begin{array}{c}
0 \\
\mathrm{I}_{\eta}
\end{array}\right)\right) \leq \eta P_{2}
\end{aligned}
$$


(17) only over a subset of the parameters $\eta, \mathbf{a}_{1}, \mathbf{a}_{2}, \mathrm{~B}_{1}, \mathrm{~B}_{2}, \mathrm{C}$ satisfying (19). In Appendixes $E$ and $F$ we present two such subsets and their corresponding achievable regions (Corollaries E.3 and F.2). In Section IV-C2, we present more general guidelines on how to choose the parameters $\eta, \mathbf{a}_{1}, \mathbf{a}_{2}, \mathrm{~B}_{1}, \mathrm{~B}_{2}$, C.

Proposition IV.5 (Monotonicity and Convergence of the Region $\mathcal{R})$ : The achievable region $\mathcal{R}\left(P_{1}, P_{2}, N, \mathrm{~K}_{W_{1} W_{2}}\right)$ satisfies the following three properties.

1) Given $P_{1}, P_{2}, N>0$, it is monotonically decreasing in $\mathrm{K}_{W_{1} W_{2}}$ with respect to the Loewner order, i.e., for positive semidefinite matrices $\mathrm{K}_{W_{1} W_{2}}$ and $\mathrm{K}_{W_{1} W_{2}}^{\prime}$ :

$$
\begin{aligned}
& \left(\mathrm{K}_{W_{1} W_{2}} \succeq \mathrm{K}_{W_{1} W_{2}}^{\prime}\right) \Longrightarrow \\
& \left(\mathcal{R}\left(P_{1}, P_{2}, N, \mathrm{~K}_{W_{1} W_{2}}\right)\right. \\
& \left.\quad \subseteq \mathcal{R}\left(P_{1}, P_{2}, N, \mathrm{~K}_{W_{1} W_{2}}^{\prime}\right)\right) .
\end{aligned}
$$

2) Given $\mathrm{K}_{W_{1} W_{2}} \succeq 0$ and $N>0$, it is continuous in $P_{1}$ and $P_{2}$, i.e., for all $P_{1}, P_{2}>0$ :

$\operatorname{cl}\left(\bigcup_{\delta>0} \mathcal{R}\left(P_{1}-\delta, P_{2}-\delta, N, \mathrm{~K}_{W_{1} W_{2}}\right)\right)$

$$
=\mathcal{R}\left(P_{1}, P_{2}, N, \mathrm{~K}_{W_{1} W_{2}}\right) \text {. }
$$

3) Given $P_{1}, P_{2}, N>0$, it converges to the perfect-feedback achievable region $\mathcal{R}\left(P_{1}, P_{2}, N, 0\right)$ as the feedback-noise variances tend to 0 irrespective of the feedback-noise correlations, i.e.,

$$
\begin{aligned}
\operatorname{cl}\left(\bigcup_{\sigma^{2}>0} \bigcap_{\mathrm{K} \succeq 0: \operatorname{tr}(\mathrm{K}) \leq \sigma^{2}} \mathcal{R}\left(P_{1}, P_{2}, N, \mathrm{~K}\right)\right) \\
=\mathcal{R}\left(P_{1}, P_{2}, N, 0\right) .
\end{aligned}
$$

\section{Proof: See Section IV-E1.}

Specializing Theorem IV.3 to symmetric channels, i.e., to $P_{1}=P_{2}=P$ and $\sigma_{1}^{2}=\sigma_{2}^{2}=\sigma^{2}$, and to $\eta=2$ and the choice of parameters $\mathbf{a}_{1}, \mathbf{a}_{2}, \mathrm{~B}_{1}, \mathrm{~B}_{2}$, and $\mathrm{C}$ presented in Section E-A in Appendix E yields the following Corollary IV.6.
Corollary IV.6 (Symmetric Noisy Feedback Channels-Suboptimal Choice of Parameters): The capacity region $\mathrm{C}_{\text {NoisyFB }}\left(P, P, N, \mathrm{~K}_{W_{1} W_{2}}\right)$ of the symmetric two-user AWGN MAC with noisy feedback, i.e., where

$$
\mathrm{K}_{W_{1} W_{2}}=\left(\begin{array}{cc}
\sigma^{2} & \sigma^{2} \varrho \\
\sigma^{2} \varrho & \sigma^{2}
\end{array}\right)
$$

contains all rate pairs $\left(R_{1}, R_{2}\right)$ satisfying the rate constraints (21a) and (21b), shown at the bottom of the page. In particular, it contains the equal-rate point $(R, R)$ whenever it satisfies (22), shown at the bottom of the page.

From Corollary IV.6 it is easily seen that the capacity of the symmetric noisy-feedback setup is larger than the no-feedback capacity, no matter how large (but finite) the feedback-noise variance $\sigma^{2}$ is. The following stronger result holds.

Theorem IV.7 (Noisy Feedback is Always Beneficial): For every feedback-noise covariance matrix $\mathrm{K}_{W_{1} W_{2}}$

$$
\mathrm{C}_{\mathrm{NoFB}}\left(P_{1}, P_{2}, N\right) \subset \mathrm{C}_{\mathrm{NoisyFB}}\left(P_{1}, P_{2}, N, \mathrm{~K}_{W_{1} W_{2}}\right)
$$

where the inclusion is strict.

Proof: Follows by Theorem V.6 ahead, which establishes that noisy partial feedback always increases capacity, and by observing that-since Transmitter 1 can always ignore its feedback - noisy feedback cannot be worse than noisy partial feedback, i.e., for all covariance matrices $\mathrm{K}_{W_{1} W_{2}}=\left(\begin{array}{cc}\sigma_{1}^{2} & \sigma_{1} \sigma_{2} \varrho \\ \sigma_{1} \sigma_{2} \varrho & \sigma_{2}^{2}\end{array}\right)$ :

$\mathrm{C}_{\text {NoisyFB }}\left(P_{1}, P_{2}, N, \mathrm{~K}_{W_{1} W_{2}}\right)$

$$
\supseteq \mathrm{C}_{\text {NoisyPartialFB }}\left(P_{1}, P_{2}, N, \sigma_{2}^{2}\right) \text {. }
$$

Specializing Theorem IV.3 to perfect feedback, i.e., $\mathrm{K}_{W_{1} W_{2}}=0$, and to the choice of parameters presented in Section F-A in Appendix F yields the following remark.

Remark IV.8 (Perfect Feedback): For the two-user AWGN MAC with perfect feedback our concatenated scheme achieves all rate pairs inside the region $\mathcal{R}_{\mathrm{Oz}}^{\rho^{*}}\left(P_{1}, P_{2}, N\right)$, i.e.,

$$
\mathcal{R}\left(P_{1}, P_{2}, N, 0\right) \supseteq \mathcal{R}_{\mathrm{Oz}}^{\rho^{*}}\left(P_{1}, P_{2}, N\right) \text {. }
$$

$$
\begin{aligned}
R_{1}, R_{2} & \leq \frac{1}{4} \log \left(1+\frac{2 P}{N}\right)+\frac{1}{4} \log \left(1+\frac{P^{2}}{(2 P+N)\left(P+N+\sigma^{2}+\frac{2 P}{N}\left(\sigma^{2}-\varrho \sigma^{2}\right)\right)}\right) \\
R_{1}+R_{2} & \leq \frac{1}{2} \log \left(1+\frac{2 P}{N}\right)+\frac{1}{4} \log \left(1+\frac{2 P^{2}}{(2 P+N)\left(P+N+\sigma^{2}+\frac{2 P}{N}\left(\sigma^{2}-\varrho \sigma^{2}\right)\right)}\right)
\end{aligned}
$$

$$
R \leq \frac{1}{4} \log \left(1+\frac{2 P}{N}\right)+\frac{1}{8} \log \left(1+\frac{2 P^{2}}{(2 P+N)\left(P+N+\sigma^{2}+\frac{2 P}{N} \sigma^{2}(1-\varrho)\right)}\right) .
$$


Proof: Is based on the specific choice of parameters in Section F-A, i.e., on the regions $\tilde{\mathcal{R}}_{\eta}\left(P_{1}, P_{2}, N, 0\right)$ in Remark F-3 in Appendix F. For details, see Section IV-E2.

We next consider the noisy-feedback setting in the asymptotic regime where the noise variances on both feedback links vanish. Proposition IV.9 shows that our achievable regions in Theorem IV. 3 converge to the point of maximum sum-rate in $\mathrm{C}_{\text {PerfectFB }}$ when the feedback-noise variances tend to zero, irrespective of the feedback-noise correlation.

Proposition IV.9 (Convergence to Maximum Sum-Rate of $\mathrm{C}_{\text {PerfectFB }}$ ): Our achievable region satisfies

$$
\begin{aligned}
& \operatorname{cl}\left(\bigcup_{\sigma^{2}>0 \mathrm{~K} \succeq 0: \operatorname{tr}(\mathrm{K}) \leq \sigma^{2}} \mathcal{R}\left(P_{1}, P_{2}, N, \mathrm{~K}\right)\right) \\
& \supseteq \mathcal{R}_{\mathrm{Oz}}^{\rho^{*}}\left(P_{1}, P_{2}, N\right) \text {. }
\end{aligned}
$$

Thus, by Remark III.7, our achievable regions in Theorem IV.3 asymptotically approach the point of maximum sum-rate in the perfect-feedback capacity region.

Proof: Follows directly by Proposition IV.5, Part 3), and by Remark IV.8.

Remark IV.10: We can strengthen Proposition IV.9, as follows. Inclusion (23) remains valid if the region $\mathcal{R}\left(P_{1}, P_{2}, N, \mathrm{~K}\right)$ is replaced by the union $\left(\bigcup_{\eta \in \mathbb{N}} \tilde{\mathcal{R}}_{\eta}\left(P_{1}, P_{2}, N, \mathrm{~K}\right)\right)$, where the regions $\tilde{\mathcal{R}}_{\eta}\left(P_{1}, P_{2}, N, \mathrm{~K}\right)$ are defined in Definition F.1 in Appendix $F$, and represent the regions achieved by our concatenated scheme for the specific choice of parameters presented in Section F-A (Appendix F).

Our last achievability result for noisy feedback is based on the rate-splitting scheme in Section IV-D1. Before stating the result in Proposition IV.13, we define the following.

Definition IV.11: For fixed $\eta \in \mathbb{N}$; fixed $\eta$-dimensional vectors $\mathbf{a}_{1}, \mathbf{a}_{2} ; \eta \times \eta$ strictly lower-triangular matrices $\mathrm{B}_{1}, \mathrm{~B}_{2}$; and $2 \times \eta$ matrix $\mathrm{C}$ define the region $\mathcal{R}_{\mathrm{RS}, 1}\left(P_{1}^{\prime}, N, \mathrm{~K}_{W_{1} W_{2}} ; \eta, \mathbf{a}_{1}, \mathbf{a}_{2}, \mathrm{~B}_{1}, \mathrm{~B}_{2}, \mathrm{C}\right)$ as the set of all rate pairs $\left(R_{1}, R_{2}\right)$ that for some nonnegative $R_{1, \mathrm{CS}}, R_{1, \mathrm{NF}}$ summing to $R_{1}$ satisfy the following two conditions:

$$
\left(R_{1, \mathrm{CS}}, R_{2}\right) \in \mathcal{R}\left(N+P_{1}^{\prime}, \mathrm{K}_{W_{1} W_{2}} ; \eta, \mathbf{a}_{1}, \mathbf{a}_{2}, \mathrm{~B}_{1}, \mathrm{~B}_{2}, \mathrm{C}\right)
$$

and

$$
R_{1, \mathrm{NF}} \leq \frac{1}{2 \eta} \log \left(\frac{\left|P_{1}^{\prime} \mathrm{I}_{\eta}+N \mathrm{I}_{\eta}+\mathrm{B}_{\mathrm{r}}\left(\mathrm{K}_{W_{1} W_{2}} \otimes \mathrm{I}_{\eta}\right) \mathrm{B}_{\mathrm{r}}^{\top}\right|}{\left|N \mathrm{I}_{\eta}+\mathrm{B}_{\mathrm{r}}\left(\mathrm{K}_{W_{1} W_{2}} \otimes \mathrm{I}_{\eta}\right) \mathrm{B}_{\mathrm{r}}^{\top}\right|}\right)
$$

where $B_{r}$ is defined in (13).

Similarly, define the region $\mathcal{R}_{\mathrm{RS}, 2}\left(P_{2}^{\prime}, N, \mathrm{~K}_{W_{1} W_{2}} ; \eta, \mathbf{a}_{1}, \mathbf{a}_{2}, \mathrm{~B}_{1}, \mathrm{~B}_{2}, \mathrm{C}\right)$ analogously to the region $\mathcal{R}_{\mathrm{RS}, 1}\left(P_{1}^{\prime}, N, \mathrm{~K}_{W_{1} W_{2}} ; \eta, \mathbf{a}_{1}, \mathbf{a}_{2}, \mathrm{~B}_{1}, \mathrm{~B}_{2}, \mathrm{C}\right)$, but with exchanged indices 1 and 2 .

Definition IV.12: Define the rate region $\mathcal{R}_{\mathrm{RS}, 1}\left(P_{1}^{\prime}, P_{1}^{\prime \prime}, P_{2}, N, \mathrm{~K}_{W_{1} W_{2}}\right) \quad$ (or for short $\left.\quad \mathcal{R}_{\mathrm{RS}, 1}\right)$ as

$$
\begin{aligned}
\mathcal{R}_{\mathrm{RS}, 1}( & \left.P_{1}^{\prime}, P_{1}^{\prime \prime}, P_{2}, N, \mathrm{~K}_{W_{1} W_{2}}\right) \\
& \triangleq \operatorname{cl}\left(\bigcup \mathcal{R}_{\mathrm{RS}, 1}\left(P_{1}^{\prime}, N, \mathrm{~K}_{W_{1} W_{2}} ; \eta, \mathbf{a}_{1}, \mathbf{a}_{2}, \mathrm{~B}_{1}, \mathrm{~B}_{2}, \mathrm{C}\right)\right)
\end{aligned}
$$

where the union is over all tuples $\left(\eta, \mathbf{a}_{1}, \mathbf{a}_{2}, \mathrm{~B}_{1}, \mathrm{~B}_{2}, \mathrm{C}\right)$ satisfying the trace constraints (19) for powers $P_{1}^{\prime \prime}$ and $P_{2}$, noise variance $\left(N+P_{1}^{\prime}\right)$, and feedback-noise covariance matrix $\mathrm{K}_{W_{1} W_{2}}$. Similarly, define the region $\mathcal{R}_{\mathrm{RS}, 2}\left(P_{1}, P_{2}^{\prime}, P_{2}^{\prime \prime}, N, \mathrm{~K}_{W_{1} W_{2}}\right.$ ) (or for short $\mathcal{R}_{\mathrm{RS}, 2}$ ) as

$$
\begin{aligned}
\mathcal{R}_{\mathrm{RS}, 2} & \left(P_{1}, P_{2}^{\prime}, P_{2}^{\prime \prime}, N, \mathrm{~K}_{W_{1} W_{2}}\right) \\
& \triangleq \operatorname{cl}\left(\bigcup \mathcal{R}_{\mathrm{RS}, 2}\left(P_{2}^{\prime}, N, \mathrm{~K}_{W_{1} W_{2}} ; \eta, \mathbf{a}_{1}, \mathbf{a}_{2}, \mathrm{~B}_{1}, \mathrm{~B}_{2}, \mathrm{C}\right)\right)
\end{aligned}
$$

where the union is over all tuples $\left(\eta, \mathbf{a}_{1}, \mathbf{a}_{2}, \mathrm{~B}_{1}, \mathrm{~B}_{2}, \mathrm{C}\right)$ satisfying the trace constraints (19) for powers $P_{1}$ and $P_{2}^{\prime \prime}$, noise variance $\left(N+P_{2}^{\prime}\right)$, and feedback-noise covariance matrix $\mathrm{K}_{W_{1} W_{2}}$.

Proposition IV.13 (Rate-Splitting for Noisy Feedback): The capacity region $\mathrm{C}_{\text {NoisyFB }}\left(P_{1}, P_{2}, N, \mathrm{~K}_{W_{1} W_{2}}\right)$ contains the region $\mathcal{R}_{\mathrm{RS}, 1}\left(P_{1}^{\prime},\left(P_{1}-P_{1}^{\prime}\right), P_{2}, N, \mathrm{~K}_{W_{1} W_{2}}\right)$ for any $P_{1}^{\prime} \in\left[0, P_{1}\right]$, and it contains the region $\mathcal{R}_{\mathrm{RS}, 2}\left(P_{1}, P_{2}^{\prime},\left(P_{2}-P_{2}^{\prime}\right), N, \mathrm{~K}_{W_{1} W_{2}}\right)$ for any $P_{2}^{\prime} \in\left[0, P_{2}\right]$ :

$$
\begin{aligned}
& \mathrm{C}_{\text {NoisyFB }}\left(P_{1}, P_{2}, N, \mathrm{~K}_{W_{1} W_{2}}\right) \\
& \quad \supseteq \bigcup_{P_{1}^{\prime} \in\left[0, P_{1}\right]} \mathcal{R}_{\mathrm{RS}, 1}\left(P_{1}^{\prime},\left(P_{1}-P_{1}^{\prime}\right), P_{2}, N, \mathrm{~K}_{W_{1} W_{2}}\right)
\end{aligned}
$$

and

$$
\begin{aligned}
& \mathrm{C}_{\text {NoisyFB }}\left(P_{1}, P_{2}, N, \mathrm{~K}_{W_{1} W_{2}}\right) \\
& \quad \supseteq \bigcup_{P_{2}^{\prime} \in\left[0, P_{2}\right]} \mathcal{R}_{\mathrm{RS}, 2}\left(P_{1}, P_{2}^{\prime},\left(P_{2}-P_{2}^{\prime}\right), N, \mathrm{~K}_{W_{1} W_{2}}\right) .
\end{aligned}
$$

Proof: The rate region is achieved by the rate-splitting scheme in Section IV-D1. The analysis is based on Theorem IV.3, on the capacity of a Gaussian multi-input antenna/multi-output antenna channel with noise sequences that are temporally-white but correlated across the antennas, and on a genie-aided argument as in [14] and [24, p.419]. The details are omitted.

Proposition IV.14 (Monotonicity and Convergence of Regions $\mathcal{R}_{\mathrm{Rs}, 1}$ and $\mathcal{R}_{\mathrm{Rs}, 2}$ ): The achievable region $\mathcal{R}_{\mathrm{RS}, 1}\left(P_{1}^{\prime}, P_{1}^{\prime \prime}, N, \mathrm{~K}_{W_{1} W_{2}}\right)$ satisfies the following three properties.

1) Given $P_{1}^{\prime}, P_{1}^{\prime \prime}, P_{2}, N>0$, it is monotonically decreasing in $\mathrm{K}_{W_{1} W_{2}}$ with respect to the Loewner order, i.e., for positive semidefinite matrices $\mathrm{K}_{W_{1} W_{2}}$ and $\mathrm{K}_{W_{1} W_{2}}^{\prime}$ :

$$
\begin{aligned}
& \left(\mathrm{K}_{W_{1} W_{2}} \succeq \mathrm{K}_{W_{1} W_{2}}^{\prime}\right) \Longrightarrow \\
& \left(\mathcal{R}_{\mathrm{RS}, 1}\left(P_{1}^{\prime}, P_{1}^{\prime \prime}, P_{2}, N, \mathrm{~K}_{W_{1} W_{2}}\right)\right. \\
& \left.\quad \subseteq \mathcal{R}_{\mathrm{RS}, 1}\left(P_{1}^{\prime}, P_{1}^{\prime \prime}, P_{2}, N, \mathrm{~K}_{W_{1} W_{2}}^{\prime}\right)\right) .
\end{aligned}
$$

2) Given $\mathrm{K}_{W_{1} W_{2}} \succeq 0$ and $N>0$, it is continuous in $P_{1}^{\prime}, P_{1}^{\prime \prime}$, and $P_{2}$, i.e., for all $P_{1}^{\prime}, P_{1}^{\prime \prime}, P_{2}>0$ :

$$
\begin{aligned}
\operatorname{cl}\left(\bigcup _ { \delta > 0 } \mathcal { R } _ { \mathrm { RS } , 1 } \left(P_{1}^{\prime}-\delta, P_{1}^{\prime \prime}\right.\right. & \left.\left.-\delta, P_{2}-\delta, N, \mathrm{~K}_{W_{1} W_{2}}\right)\right) \\
& =\mathcal{R}_{\mathrm{RS}, 1}\left(P_{1}^{\prime}, P_{1}^{\prime \prime}, P_{2}, N, \mathrm{~K}_{W_{1} W_{2}}\right) .
\end{aligned}
$$

3) Given $P_{1}^{\prime}, P_{1}^{\prime \prime}, P_{2}, N>0$, it converges to the perfect-feedback achievable region $\mathcal{R}_{\mathrm{RS}, 1}\left(P_{1}^{\prime}, P_{1}^{\prime \prime}, P_{2}, N, 0\right)$ 
as the feedback-noise variances tend to 0 irrespective of the feedback-noise correlations, i.e.,

$$
\begin{aligned}
\operatorname{cl}\left(\bigcup_{\sigma^{2}>0 \mathrm{~K} \succeq 0: \operatorname{tr}(\mathrm{K}) \leq \sigma^{2}} \mathcal{R}_{\mathrm{RS}, 1}\left(P_{1}^{\prime}, P_{1}^{\prime \prime}, P_{2}, N, \mathrm{~K}\right)\right) \\
=\mathcal{R}_{\mathrm{RS}, 1}\left(P_{1}^{\prime}, P_{1}^{\prime \prime}, P_{2}, N, 0\right) .
\end{aligned}
$$

Similarly, for $\mathcal{R}_{\mathrm{RS}, 2}\left(P_{1}, P_{2}^{\prime}, P_{2}^{\prime \prime}, N, \mathrm{~K}_{W_{1} W_{2}}\right)$.

Proof: Follows from Proposition IV.5 and because for fixed $\mathbf{a}_{1}, \mathbf{a}_{2}, \mathrm{~B}_{1}, \mathrm{~B}_{2}$, and $\mathrm{C}$ the RHS of (24) satisfies the following three properties. It is monotonically decreasing in $\mathrm{K}_{W_{1} W_{2}}$ with respect to the Loewner order, it is continuous in $P_{1}^{\prime}$, and it converges to $\frac{1}{2} \log \left(1+\frac{P_{1}^{\prime}}{N}\right)$ as the feedback-noise variances tend to 0 irrespective of the feedback-noise correlations. The details are omitted.

With the rate-splitting extension in Section IV-D1 and Propositions IV.13 and IV.14, Remark IV.8 and Proposition IV.9 can be generalized to all the boundary points of the capacity region $\mathrm{C}_{\text {PerfectFB. }}$

Remark IV.15 (Perfect Feedback): For the two-user AWGN MAC with perfect feedback our rate-splitting scheme in Section IV-D1 achieves all rate pairs in Ozarow's perfect-feedback capacity region $\mathrm{C}_{\text {PerfectFB }}\left(P_{1}, P_{2}, N\right)$ :

$$
\begin{aligned}
& \mathrm{C}_{\text {PerfectFB }}\left(P_{1}, P_{2}, N\right) \\
&=\left(\bigcup_{P_{1}^{\prime} \in\left[0, P_{1}\right]} \mathcal{R}_{\mathrm{RS}, 1}\left(P_{1}^{\prime},\left(P_{1}-P_{1}^{\prime}\right), P_{2}, N, 0\right)\right) \\
& \cup\left(\bigcup_{P_{2}^{\prime} \in\left[0, P_{2}\right]} \mathcal{R}_{\mathrm{RS}, 2}\left(P_{1}, P_{2}^{\prime},\left(P_{2}-P_{2}^{\prime}\right), N, 0\right)\right) .
\end{aligned}
$$

In fact, for each $\rho \in\left[0, \rho^{*}\right]$ there exists a $P_{1}^{\prime}(\rho) \in\left[0, P_{1}\right]$ so that

$$
\begin{aligned}
& \mathcal{R}_{\mathrm{RS}, 1}\left(P_{1}^{\prime}(\rho),\left(P_{1}-P_{1}^{\prime}(\rho)\right), P_{2}, N, 0\right) \\
& \supseteq \mathcal{R}_{1, \mathrm{Oz}}^{\rho}\left(P_{1}, P_{2}, N\right)
\end{aligned}
$$

and a $P_{2}^{\prime}(\rho) \in\left[0, P_{2}\right]$ so that

$$
\begin{aligned}
& \mathcal{R}_{\mathrm{RS}, 2}\left(P_{1}, P_{2}^{\prime}(\rho),\left(P_{2}-P_{2}^{\prime}(\rho)\right), N, 0\right) \\
& \supseteq \mathcal{R}_{2, \mathrm{Oz}}^{\rho}\left(P_{1}, P_{2}, N\right) .
\end{aligned}
$$

Proof: By Remark III.7, (25) follows directly from (26). For a proof of (26), see Section IV-E3.

Proposition IV.16 (Convergence to Boundary of $\mathrm{C}_{\text {PerfectFB }}$ ): For every $\rho \in\left[0, \rho^{*}\left(P_{1}, P_{2}, N\right)\right]$ we can find some $P_{1}^{\prime}(\rho) \in$ $\left[0, P_{1}\right]$ so that

$$
\begin{array}{r}
\operatorname{cl}\left(\bigcup_{\sigma^{2}>0} \bigcap_{\substack{\mathrm{K} \succeq 0: \\
\operatorname{tr}(\mathrm{K}) \leq \sigma^{2}}} \mathcal{R}_{\mathrm{RS}, 1}\left(P_{1}^{\prime}(\rho),\left(P_{1}-P_{1}^{\prime}(\rho)\right), P_{2}, N, \mathrm{~K}\right)\right) \\
\supseteq \mathcal{R}_{1, \mathrm{Oz}}^{\rho}\left(P_{1}, P_{2}, N\right) .
\end{array}
$$

Similarly, for every $\rho \in\left[0, \rho^{*}\left(P_{1}, P_{2}, N\right)\right]$ we can find some $P_{2}^{\prime}(\rho) \in\left[0, P_{2}\right]$ so that

$$
\begin{array}{r}
\operatorname{cl}\left(\bigcup_{\sigma^{2}>0} \bigcap_{\substack{\mathrm{K} \succeq 0: \\
\operatorname{tr}(\mathrm{K}) \leq \sigma^{2}}} \mathcal{R}_{\mathrm{RS}, 2}\left(P_{1}, P_{2}^{\prime}(\rho),\left(P_{2}-P_{2}^{\prime}(\rho)\right), N, \mathrm{~K}\right)\right) \\
\supseteq \mathcal{R}_{2, \mathrm{Oz}}^{\rho}\left(P_{1}, P_{2}, N\right) .
\end{array}
$$

Thus, by Remark III.5 and Definition III.6, our achievable regions in Proposition IV.13 asymptotically approach all boundary points of the perfect-feedback capacity region.

Proof: See Section IV-E4.

Propositions IV.13 and IV.16, combined with Remark III.7, yield the following continuity result.

Theorem IV.17 (Continuity of Noisy-Feedback Capacity Region): For all $P_{1}, P_{2}, N>0$ :

$$
\begin{aligned}
\operatorname{cl}\left(\bigcup_{\sigma^{2}>0 \mathrm{~K} \succeq 0: \operatorname{tr}(\mathrm{K}) \leq \sigma^{2}} \mathrm{C}_{\text {NoisyFB }}\left(P_{1}, P_{2}, N, \mathrm{~K}\right)\right) \\
=\mathrm{C}_{\text {PerfectFB }}\left(P_{1}, P_{2}, N\right) .
\end{aligned}
$$

Proof: See Section IV-E5 for details.

\section{B. Simple Scheme}

We present a simple coding scheme for the noisy-feedback setting. It is a special case of the concatenated scheme in Section IV-C1: the simple scheme with parameters $a_{1,1}, a_{1,2}, a_{2,1}, a_{2,2}, b_{1}, b_{2}$ coincides with the concatenated scheme for noisy feedback with parameters $\eta=2, \mathbf{a}_{1}=\left(\begin{array}{ll}a_{1,1} & a_{2,1}\end{array}\right)^{\top}, \mathbf{a}_{2}=\left(\begin{array}{ll}a_{2,1} & a_{2,2}\end{array}\right)^{\top}$, $\mathrm{B}_{1}=\left(\begin{array}{cc}0 & 0 \\ b_{1} & 0\end{array}\right), \mathrm{B}_{2}=\left(\begin{array}{cc}0 & 0 \\ b_{2} & 0\end{array}\right), \mathrm{C}=\mathrm{I}_{2}$. We present the simple scheme here separately, because it is easier and yet powerful enough to establish Corollary IV.6 and Theorem IV.7.

Prior to communication a blocklength- $n$, rate- $R_{1}$ codebook $\mathcal{C}_{1}$ and a blocklength- $n$, rate- $R_{2}$ codebook $\mathcal{C}_{2}$ are generated and revealed to both transmitters and to the receiver. The codewords of codebook $\mathcal{C}_{1}$ are chosen independently with the $n$ components $\Xi_{1,1}\left(m_{1}\right), \ldots, \Xi_{1, n}\left(m_{1}\right)$ of the $m_{1}$ th codeword chosen IID zero-mean unit-variance Gaussian. The codebook $\mathcal{C}_{2}$ is drawn similarly. Messages $M_{1}$ and $M_{2}$ are then transmitted over $2 n$ channel uses by sending each symbol of the $n$-length codewords $\Xi_{1}^{n}\left(M_{1}\right)$ and $\Xi_{2}^{n}\left(M_{2}\right)$ over two consecutive channel uses. More precisely, at odd time steps $t=2(k-1)+1$, for $k \in\{1, \ldots, n\}$, Transmitter 1 sends

$$
X_{1,2(k-1)+1}=a_{1,1} \Xi_{1, k}
$$

and Transmitter 2 sends

$$
X_{2,2(k-1)+1}=a_{2,1} \Xi_{2, k} \text {. }
$$

At even time steps $t=2 k$, for $k \in\{1, \ldots, n\}$, Transmitter 1 sends

$$
X_{1,2 k}=a_{1,2} \Xi_{1, k}-b_{1} V_{1,2(k-1)+1}
$$


and Transmitter 2 sends

$$
X_{2,2 k}=a_{2,2} \Xi_{2, k}-b_{2} V_{2,2(k-1)+1} .
$$

To ensure that the two input sequences $\left\{X_{1, t}\right\}_{t=1}^{2 n}$ and $\left\{X_{2, t}\right\}_{t=1}^{2 n}$ satisfy the power constraints (4), the parameters $a_{1,1}, a_{1,2}, a_{2,1}, a_{2,2}, b_{1}$, and $b_{2}$ are chosen as to simultaneously satisfy

$$
a_{1,1}^{2}+\left(a_{1,2}-b_{1} a_{1,1}\right)^{2}+b_{1}^{2}\left(a_{2,1}^{2}+N+\sigma_{1}^{2}\right) \leq 2 P_{1}
$$

and

$$
a_{2,1}^{2}+\left(a_{2,2}-b_{2} a_{2,1}\right)^{2}+b_{2}^{2}\left(a_{1,1}^{2}+N+\sigma_{2}^{2}\right) \leq 2 P_{2} .
$$

The receiver uses an optimal decoding rule to decode Messages $M_{1}$ and $M_{2}$ based on the observed sequence of channel outputs $Y_{1}, \ldots, Y_{2 n}$.

To describe the performance of the scheme, let $\Xi_{1}, \Xi_{2}, Z_{\text {odd }}$, and $Z_{\text {even }}$ be independent zero-mean Gaussian random variables, where $\Xi_{1}$ and $\Xi_{2}$ are of variance 1 and $Z_{\text {odd }}$ and $Z_{\text {even }}$ of variance $N$. Independent thereof, let the pair $\left(W_{1}, W_{2}\right)$ be a zero-mean bivariate Gaussian of covariance matrix $\mathrm{K}_{W_{1} W_{2}}$ as defined in (1). Also, let $Y_{\text {odd }}$ and $Y_{\text {even }}$ be defined as

$$
\begin{aligned}
Y_{\text {odd }} \triangleq & a_{1,1} \Xi_{1}+a_{2,1} \Xi_{2}+Z_{\text {odd }}, \\
Y_{\text {even }} \triangleq & a_{1,2} \Xi_{1}+a_{2,2} \Xi_{2}-b_{1} V_{1, \text { odd }} \\
& -b_{2} V_{2, \text { odd }}+Z_{\text {even }} ;
\end{aligned}
$$

and $V_{1 \text {,odd }}$ and $V_{2 \text {,odd }}$ be defined as

$$
V_{\nu, \text { odd }} \triangleq Y_{\text {odd }}+W_{\nu}, \quad \nu \in\{1,2\} .
$$

The performance of the simple scheme is then described as follows. The scheme achieves all nonnegative rate pairs $\left(R_{1}, R_{2}\right)$ that simultaneously satisfy

$$
\begin{aligned}
R_{1} & \leq \frac{1}{2} I\left(\Xi_{1} ; Y_{\text {odd }}, Y_{\text {even }} \mid \Xi_{2}\right), \\
R_{2} & \leq \frac{1}{2} I\left(\Xi_{2} ; Y_{\text {odd }}, Y_{\text {even }} \mid \Xi_{1}\right), \\
R_{1}+R_{2} & \leq \frac{1}{2} I\left(\Xi_{1}, \Xi_{2} ; Y_{\text {odd }}, Y_{\text {even }}\right)
\end{aligned}
$$

or, equivalently, as obtained by evaluating the mutual information expressions on the RHSs of (33), it achieves all nonnegative rate pairs $\left(R_{1}, R_{2}\right)$ that simultaneously satisfy

$$
\begin{aligned}
R_{1} \leq \frac{1}{4} \log (1 & +\frac{a_{1,1}^{2}}{N} \\
& \left.+\frac{a_{1,2}^{2}}{b_{1}^{2} \sigma_{1}^{2}+b_{2}^{2} \sigma_{2}^{2}+2 b_{1} b_{2} \varrho \sigma_{1} \sigma_{2}+N}\right) \\
R_{2} \leq \frac{1}{4} \log (1 & +\frac{a_{2,1}^{2}}{N} \\
& \left.+\frac{a_{2,2}^{2}}{b_{1}^{2} \sigma_{1}^{2}+b_{2}^{2} \sigma_{2}^{2}+2 b_{1} b_{2} \varrho \sigma_{1} \sigma_{2}+N}\right)
\end{aligned}
$$

and

$$
\begin{aligned}
R_{1}+R_{2} & \\
\leq \frac{1}{4} \log (1 & +\frac{a_{1,1}^{2}+a_{2,1}^{2}}{N} \\
& +\frac{a_{1,2}^{2}+a_{2,2}^{2}}{b_{1}^{2} \sigma_{1}^{2}+b_{2}^{2} \sigma_{2}^{2}+2 b_{1} b_{2} \varrho \sigma_{1} \sigma_{2}+N} \\
& \left.+\frac{\left(a_{1,1} a_{2,2}-a_{2,1} a_{1,2}\right)^{2}}{N\left(b_{1}^{2} \sigma_{1}^{2}+b_{2}^{2} \sigma_{2}^{2}+2 b_{1} b_{2} \varrho \sigma_{1} \sigma_{2}+N\right)}\right)
\end{aligned}
$$

for some choice of the parameters $a_{1,1}, a_{1,2}, a_{2,1}, a_{2,2}, b_{1}, b_{2}$ satisfying (32).

\section{Concatenated Scheme}

We first present our concatenated coding scheme with general parameters in Section IV-C1; in Section IV-C2 we then give guidelines on how to choose the parameters of this concatenated scheme.

1) Scheme: We propose an encoding scheme with a concatenated structure where each of the encoders and the decoder consists of an outer part and an inner part. (Here the inner parts are the parts that are closer to the physical channel, see Fig. 6.) In our scheme the various parts fulfill the following tasks. The outer encoders map the messages into codewords (without using the feedback) and feed these codewords to their corresponding inner encoders. The inner encoders produce for every fed symbol a sequence of $\eta$ channel inputs to the MAC with feedback, for some positive integer $\eta$. In particular, when fed the symbol $\xi_{1} \in \mathbb{R}$, Inner Encoder 1 produces $\eta$ inputs which depend on $\xi_{1}$ and on the observed feedback outputs; all symbols fed to the inner encoder are treated in the same way. Inner Encoder 2 is analogously defined. The $\eta$ symbols which the MAC outputs for every pair of input symbols $\left(\xi_{1}, \xi_{2}\right)$ are then linearly mapped by the inner decoder to a pair of estimates $\left(\hat{\Xi}_{1}, \hat{\Xi}_{2}\right)$, and the estimates are fed to the outer decoder. Thus, the outer decoder is fed with a vector in $\mathbb{R}^{2}$ every $\eta$ channel uses. Based on the sequence of vectors produced by the inner decoder, the outer decoder then decodes the transmitted messages.

Consequently, the inner encoders and the inner decoder transform each subblock of $\eta$ channel uses of the original MAC into a single channel use of a "new" time-invariant and memoryless MAC which for given inputs $\xi_{1} \in \mathbb{R}$ and $\xi_{2} \in \mathbb{R}$ produces the channel output $\left(\hat{\Xi}_{1}, \hat{\Xi}_{2}\right)^{\top} \in \mathbb{R}^{2}$. We denote the new MAC by $\xi_{1}, \xi_{2} \mapsto\left(\hat{\Xi}_{1}, \hat{\Xi}_{2}\right)$. We can then think of the overall scheme as a no-feedback scheme over the new MAC $\xi_{1}, \xi_{2} \mapsto\left(\hat{\Xi}_{1}, \hat{\Xi}_{2}\right)$. As a consequence, the capacity of the original MAC with feedback, which we denote by $x_{1}, x_{2} \mapsto Y$, is inner bounded by the capacity of the new MAC $\xi_{1}, \xi_{2} \mapsto\left(\hat{\Xi}_{1}, \hat{\Xi}_{2}\right)$ without feedback but scaled by $\eta^{-1}$ to account for the fact that to send the symbols $\xi_{1}, \xi_{2}$ over the new MAC the original channel is used $\eta$ times.

We first sketch some of the properties of the inner encoders and the inner decoder and postpone their detailed description to after the description of the outer encoders and decoder. We choose the inner encoders and the inner decoder so that the MAC $\xi_{1}, \xi_{2} \mapsto\left(\hat{\Xi}_{1}, \hat{\Xi}_{2}\right)$ can be described by

$$
\left(\begin{array}{c}
\hat{\Xi}_{1} \\
\hat{\Xi}_{2}
\end{array}\right)=\mathrm{A}\left(\begin{array}{l}
\xi_{1} \\
\xi_{2}
\end{array}\right)+\mathbf{T}
$$




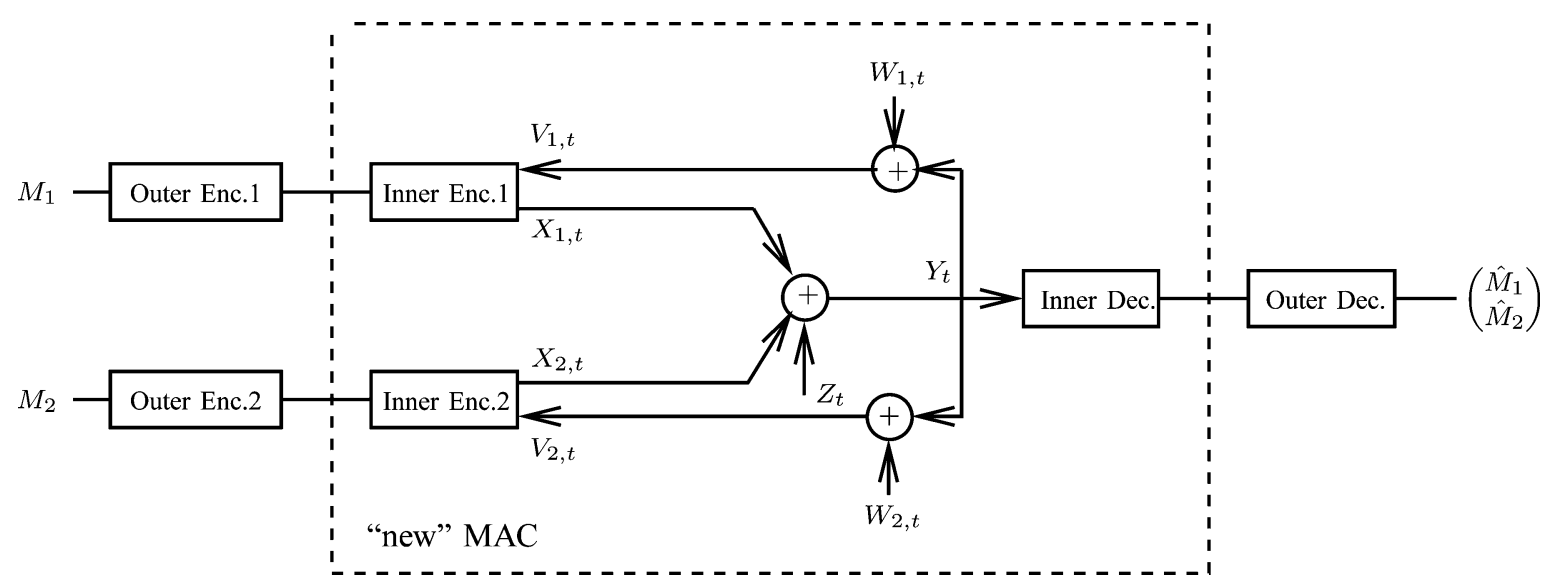

Fig. 6. Structure of concatenated scheme.

where $A$ is a deterministic $2 \times 2$ matrix and where $\mathbf{T}$ is a bivariate Gaussian whose law does not depend on the pair of inputs $\left(\xi_{1}, \xi_{2}\right)$. Also, the inner encoders are designed so that if both outer encoders satisfy a unit average block-power constraint (over time and messages) and if at every epoch the symbols produced by the outer encoders are zero-mean (when averaged over the messages), then the channel inputs to the original MAC $x_{1}, x_{2} \mapsto Y$ satisfy the average power constraints (4).

For the outer code (encoders and decoder) we choose a capacity achieving zero-mean code for the MAC $\xi_{1}, \xi_{2} \mapsto$ $\left(\hat{\Xi}_{1}, \hat{\Xi}_{2}\right)$ under an average block-power constraint of 1 . Note that there is no loss in optimality in restricting ourselves to zero-mean codes because subtracting the mean of the code can only reduce its average power (averaged over time and messages) and does not change the performance on an additive noise MAC such as (34). We shall need the property that the outer encoders produce zero-mean symbols in the power-analysis of the input sequences to the original channel $x_{1}, x_{2} \mapsto Y$.

For the inner encoders and the inner decoder we choose linear mappings. To obtain a compact description of the linear mappings we stack the $\eta$ channel inputs, $X_{\nu, 1}, \ldots, X_{\nu, \eta}$, produced by Inner Encoder $\nu$ in an $\eta$-dimensional column vector

$$
\mathbf{X}_{\nu} \triangleq\left(X_{\nu, 1}, \ldots, X_{\nu, \eta}\right)^{\top}, \quad \nu \in\{1,2\}
$$

and similarly we stack the $\eta$ feedback outputs, $V_{\nu, 1}, \ldots, V_{\nu, \eta}$, observed by Inner Encoder $\nu$ in the $\eta$-dimensional vector

$$
\mathbf{V}_{\nu}=\left(V_{\nu, 1}, \ldots, V_{\nu, \eta}\right)^{\top}, \quad \nu \in\{1,2\} .
$$

We can then describe our choice of the inner encoders as follows. When fed the input symbol $\xi_{\nu} \in \mathbb{R}$, Inner Encoder $\nu$ produces

$$
\mathbf{X}_{\nu}=\mathbf{a}_{\nu} \xi_{\nu}+\mathrm{B}_{\nu} \mathbf{V}_{\nu}, \quad \nu \in\{1,2\}
$$

where $\mathrm{a}_{\nu}$ are $\eta$-dimensional column vectors and $\mathrm{B}_{\nu}$ are $\eta \times \eta$ matrices which are strictly lower-triangular (because the feedback is causal). Also, as previously mentioned, we restrict the inner encoders to produce sequences of inputs to the original MAC $x_{1}, x_{2} \mapsto Y$ that satisfy the average block-power constraints (4) when the outer encoders feed them with zero-mean sequences of unit average block-power. By (35) this is the case whenever the trace constraints (19) are satisfied. Thus, in the following we only allow for vectors $\mathbf{a}_{1}$ and $\mathbf{a}_{2}$ and for strictly lower-triangular matrices $B_{1}$ and $B_{2}$ satisfying (19).

To describe our linear choice of the inner decoder, we stack the $\eta$ outputs $Y_{1}, \ldots, Y_{\eta}$, which the original MAC produces for the pairs of inputs $\left(X_{1,1}, X_{2,1}\right), \ldots,\left(X_{1, \eta}, X_{2, \eta}\right)$, into the $\eta$-dimensional column vector

$$
\mathbf{Y} \triangleq\left(Y_{1}, \ldots, Y_{\eta}\right)^{\top} .
$$

We can then express the estimates produced by the outer decoder by

$$
\left(\begin{array}{c}
\hat{\Xi}_{1} \\
\hat{\Xi}_{2}
\end{array}\right)=\mathrm{DY}
$$

for some matrix of our choice $D \in \mathbb{R}^{2 \times \eta}$.

In the following we describe the MAC $\xi_{1}, \xi_{2} \mapsto\left(\hat{\Xi}_{1}, \hat{\Xi}_{2}\right)$ as induced by $\eta, \mathbf{a}_{1}, \mathbf{a}_{2}, \mathrm{~B}_{1}, \mathrm{~B}_{2}$, and D. Given inputs $\xi_{1}, \xi_{2} \in \mathbb{R}$, it produces the vector of estimates

$$
\left(\begin{array}{c}
\hat{\Xi}_{1} \\
\hat{\Xi}_{2}
\end{array}\right)=\mathrm{A}\left(\begin{array}{l}
\xi_{1} \\
\xi_{2}
\end{array}\right)+\mathbf{T}
$$

where the $2 \times 2$-matrix $A$ is given by

$$
\mathrm{A}=\mathrm{D}\left(\mathrm{I}_{\eta}-\left(\mathrm{B}_{1}+\mathrm{B}_{2}\right)\right)^{-1} \mathrm{~A}_{\mathrm{r}}
$$

where $A_{r}$ is defined in (11), and where the noise vector $\mathbf{T}$ is a zero-mean bivariate Gaussian

$$
\mathbf{T}=\mathrm{D}\left(\mathrm{I}_{\eta}-\left(\mathrm{B}_{1}+\mathrm{B}_{2}\right)\right)^{-1}\left(\mathrm{~B}_{1} \mathbf{W}_{1}+\mathrm{B}_{2} \mathbf{W}_{2}+\mathbf{Z}\right)
$$

for $\mathbf{W}_{1} \triangleq\left(W_{1,1}, \ldots, W_{1, \eta}\right)^{\top}, \mathbf{W}_{2} \triangleq\left(W_{2,1}, \ldots, W_{2, \eta}\right)^{\top}$, and $\mathbf{Z} \triangleq\left(Z_{1}, \ldots, Z_{\eta}\right)^{\mathrm{T}}$. (Notice, that since $\mathrm{B}_{1}$ and $\mathrm{B}_{2}$ are strictly lower-triangular matrices, the matrix $\left(\mathrm{I}_{\eta}-\left(\mathrm{B}_{1}+\mathrm{B}_{2}\right)\right)$ is nonsingular and the inverse exists.) Defining the $2 \times \eta$ matrix

$$
\mathrm{C} \triangleq \mathrm{D}\left(\mathrm{I}_{\eta}-\left(\mathrm{B}_{1}+\mathrm{B}_{2}\right)\right)^{-1}
$$

we can express the matrix $A$ in (38) as

$$
\mathrm{A}=\mathrm{CA}_{\mathrm{r}}
$$

and the noise vector in (39) as

$$
\mathbf{T}=\mathrm{C}\left(\mathrm{B}_{1} \mathbf{W}_{1}+\mathrm{B}_{2} \mathbf{W}_{2}+\mathbf{Z}\right) .
$$


For fixed $\eta, \mathrm{B}_{1}, \mathrm{~B}_{2}$, the mapping (40) from $\mathrm{D}$ to $\mathrm{C}$ is one-toone, and thus we can parameterize our concatenated scheme for noisy feedback by the parameters $\eta, \mathbf{a}_{1}, \mathbf{a}_{2}, \mathrm{~B}_{1}, \mathrm{~B}_{2}, \mathrm{C}$.

Note that by choosing $\eta=1, a_{1}=\sqrt{P_{1}}, a_{2}=\sqrt{P_{2}}$, and $\mathrm{C}$ as the $2 \times 1$ matrix with unit entries, our scheme reduces to the capacity-achieving scheme for the original MAC $x_{1}, x_{2} \mapsto Y$ without feedback subject to the power constraints (4).

2) Choice of Parameters: Given channel parameters $P_{1}, P_{2}, \quad N, \mathrm{~K}_{W_{1} W_{2}}$, determining for each rate pair in $\mathcal{R}\left(P_{1}, P_{2}, N, \mathrm{~K}_{W_{1} W_{2}}\right)$ a set of parameters $\eta, \mathbf{a}_{1}, \mathbf{a}_{2}, \mathrm{~B}_{1}, \mathrm{~B}_{2}, \mathrm{C}$ that achieves this rate pair seems to be analytically intractable. Instead, we present guidelines on how to choose parameters and discuss the two choices of parameters in Section E-A (Appendix E) and Section F-A (Appendix F) that lead to Corollary IV.6 and Proposition IV.8.

For the purpose of describing our guidelines, throughout this section, we replace the symbols $\xi_{1}$ and $\xi_{2}$ fed to the inner encoders by the independent standard Gaussians $\Xi_{1}$ and $\Xi_{2}$.

We start with the matrix $C$. Given parameters $\eta, \mathbf{a}_{1}, \mathbf{a}_{2}, \mathrm{~B}_{1}$, and $B_{2}$, the matrix $C$ should be chosen as $C=C_{\text {LMMSE }}$, where

$$
\mathrm{C}_{\mathrm{LMMSE}} \triangleq \mathrm{A}_{\mathrm{r}}^{\top}\left(\mathrm{A}_{\mathrm{r}} \mathrm{A}_{\mathrm{r}}^{\top}+N \mathrm{I}_{\eta}+\mathrm{B}_{\mathrm{r}}\left(\mathrm{K}_{W_{1} W_{2}} \otimes \mathrm{I}_{\eta}\right) \mathrm{B}_{\mathrm{r}}^{\top}\right)^{-1}
$$

By (36), (41), and (42) this choice implies that

$$
\left(\begin{array}{c}
\hat{\Xi}_{1} \\
\hat{\Xi}_{2}
\end{array}\right)=\mathrm{E}\left[\left(\begin{array}{c}
\Xi_{1} \\
\Xi_{2}
\end{array}\right) \mid Y_{1}, \ldots, Y_{\eta}\right]
$$

and hence we call the matrix $C_{\text {LMMSE }}$ the LMMSE-estimation matrix. The choice $\mathrm{C}=\mathrm{C}_{\mathrm{LMMSE}}$ is optimal in the sense that the corresponding region $\mathcal{R}\left(N, \mathrm{~K}_{W_{1} W_{2}} ; \eta, \mathbf{a}_{1}, \mathbf{a}_{2}, \mathrm{~B}_{1}, \mathrm{~B}_{2}, \mathrm{C}_{\text {LMMSE }}\right)$ contains all regions $\mathcal{R}\left(N, \mathrm{~K}_{W_{1} W_{2}} ; \eta, \mathbf{a}_{1}, \mathbf{a}_{2}, \mathrm{~B}_{1}, \mathrm{~B}_{2}, \mathrm{C}\right)$ that correspond to other choices of $C$. The optimality of the LMMSE-estimation matrix can be argued as follows. When (44) holds, then even additionally revealing $\mathbf{Y}$ (or any linear combinations thereof) to the outer decoder does not increase the set of achievable rates in our scheme. Obviously, choosing $C=U C_{\text {LMMSE }}$ for any nonsingular 2-by-2 matrix $U$ is also optimal. In particular, when $\eta=2$ every nonsingular matrix is an optimal choice for C.

We next consider the choice of parameters $\mathbf{a}_{1}, \mathbf{a}_{2}, \mathrm{~B}_{1}, \mathrm{~B}_{2}$ and first focus on the special case of perfect feedback. This special case is in view of Ozarow's capacity result [13] only of limited interest, but it provides insight on how to choose the parameters for other settings, e.g., the perfect partial-feedback setting (see Section V-C2) and the noisy feedback-setting with receiver side-information (Section VI-B2).

For perfect feedback and a fixed $\eta$, the parameters $\mathbf{a}_{1}, \mathbf{a}_{2}, \mathrm{~B}_{1}, \mathrm{~B}_{2}$ should be chosen such that Inner Encoder $\nu$, for $\nu \in\{1,2\}$, produces as its $\ell$ th channel input a scaled version of the LMMSE-estimation error of $\Xi_{\nu}$ when observing $\left(Y_{1}, \ldots, Y_{\ell-1}\right)$, i.e.

$$
X_{1, \ell}=\pi_{1, \ell}\left(\Xi_{1}-\mathrm{E}\left[\Xi_{1} \mid Y^{\ell-1}\right]\right), \ell \in\{1, \ldots, \eta\}
$$

and

$$
X_{2, \ell}=\pi_{2, \ell}\left(\Xi_{2}-\mathrm{E}\left[\Xi_{2} \mid Y^{\ell-1}\right]\right), \ell \in\{1, \ldots, \eta\} \text { (45b) }
$$

for some real numbers $\pi_{1,1}, \ldots, \pi_{1, \eta}$ and $\pi_{2,1}, \ldots, \pi_{2, \eta}$. In fact, every choice of parameters not satisfying (45) can be strictly improved (with an appropriate choice of $\mathrm{C}$ ) so as to achieve a larger region (see Appendix C).

For general noisy feedback, it is not optimal to choose $\mathbf{a}_{1}, \mathbf{a}_{2}, \mathrm{~B}_{1}, \mathrm{~B}_{2}$ as in (45) when the channel outputs $Y_{1}, \ldots, Y_{\ell-1}$ are replaced by the feedback outputs $V_{1,1}, \ldots, V_{1, \ell-1}$ and $V_{2,1}, \ldots, V_{2, \ell-1}$. Intuitively, the reason is that with such a choice the inner encoders introduce too much feedback noise into the forward communication.

For the general setup it seems infeasible to derive the set of optimal parameters $\mathbf{a}_{1}, \mathbf{a}_{2}, \mathrm{~B}_{1}, \mathrm{~B}_{2}$. However, it is easily proved that the parameters $\mathbf{a}_{1}, \mathbf{a}_{2}, \mathrm{~B}_{1}, \mathrm{~B}_{2}$ have to be chosen so that they satisfy both power constraints (19a) and (19b) with equality, since otherwise there exists a choice of parameters which corresponds to a larger achievable region. This readily follows from the alternative formulation of $\mathcal{R}\left(N, \mathrm{~K}_{W_{1} W_{2}} ; \eta, \mathbf{a}_{1}, \mathbf{a}_{2}, \mathrm{~B}_{1}, \mathrm{~B}_{2}, \mathrm{C}\right)$ in Section D-A (Appendix D), because the RHSs of (148) (which determine $\left.\mathcal{R}\left(N, \mathrm{~K}_{W_{1} W_{2}} ; \eta, \mathbf{a}_{1}, \mathbf{a}_{2}, \mathrm{~B}_{1}, \mathrm{~B}_{2}, \mathrm{C}\right)\right)$ can always be increased by changing the last entry of $\mathbf{a}_{1}$, i.e., $a_{1, \eta}$, or the last entry of $\mathbf{a}_{2}$, i.e., $a_{2, \eta}$.

We finally consider the choice of $\eta$. If the goal is to maximize the single rates, it is trivially optimal to choose $\eta=1$ irrespective of the channel parameters $P_{1}, P_{2}, N, \mathrm{~K}_{W_{1} W_{2}}$. If in contrast the goal is to maximize the sum-rate it seems infeasible to derive the optimal $\eta$. However, numerical results indicate that the larger the feedback-noise variances are, the smaller the parameter $\eta$ should be chosen. It is easily proved that in the extreme case of no feedback the sum-rate is maximized by choosing $\eta=1$. In contrast, in the extreme case of perfect feedback we prove in Section IV-E2 that with the choice of parameters suggested in Section F-A (Appendix F) the maximum sum-rate of our concatenated scheme converges to the perfect-feedback sum-rate capacity as the parameter $\eta$ tends to infinity.

In the remaining, we discuss the two specific choices of the parameters $\mathbf{a}_{1}, \mathbf{a}_{2}, \mathrm{~B}_{1}, \mathrm{~B}_{2}, \mathrm{C}$ given $\eta \in \mathbb{N}$ presented in Section E-A (Appendix E) and in Section F-A (Appendix F). For both choices, the parameter $C$ is the LMMSE-estimation matrix and the parameters $\mathbf{a}_{1}, \mathbf{a}_{2}, \mathrm{~B}_{1}, \mathrm{~B}_{2}$ are such that when specialized to perfect feedback they satisfy (45). In the choice in Section E-A, each inner encoder allocates the same power for all channel inputs. The achievable region corresponding to this choice is presented in Corollary E.3, and includes as special case the result on the symmetric setup in Corollary IV.6. In the choice in Section F-A, the inner encoders use the power-allocation strategy suggested by [11] for perfect feedback. The corresponding achievable region is presented in Corollary F.2, and includes as special case the achievable region for perfect feedback in Remark F.3 used in the proof of Propositions IV.9 and IV.16 and Theorem IV.17.

\section{Extensions of the Concatenated Scheme}

In the following three subsections we present three extensions of our concatenated scheme by rate-splitting it with other schemes. The idea of rate-splitting was introduced in [2] and [13]. 
1) Rate-Splitting With No-Feedback Scheme: In this first extension we combine our scheme with a no-feedback scheme employing IID Gaussian codewords. This extension was inspired by the rate-splitting scheme proposed by Ozarow for perfect feedback [13]. Only one transmitter applies the rate-splitting. For the description we assume it is Transmitter 1. Thus, Transmitter 1 splits Message $M_{1}$ of rate $R_{1}$ into two independent parts: Message $M_{1, \mathrm{NF}}$ of rate $R_{1, \mathrm{NF}}$ and Message $M_{1, \mathrm{CS}}$ of rate $R_{1, \mathrm{CS}}$, where $R_{1, \mathrm{NF}}$ and $R_{1, \mathrm{CS}}$ sum up to $R_{1}$. Here, NF stands for "no-feedback" and CS stands for "concatenated scheme."

We first present a rough overview of the scheme. We start with the encodings. Transmitter 1 uses a fraction of its available power $P_{1}^{\prime}$, for some $0 \leq P_{1}^{\prime} \leq P_{1}$, to produce a sequence by encoding Message $M_{1, \mathrm{NF}}$ using Gaussian codewords 9 (without using the feedback). With the rest of the power $\left(P_{1}-P_{1}^{\prime}\right)$ it produces a sequence of the same length by encoding Message $M_{1, \mathrm{CS}}$ using our concatenated scheme and the outputs of the feedback link. It sends the sum of the two produced sequences over the channel. If the concatenated scheme is of parameter $\eta$ and its outer code is of blocklength $n$, then both sequences are of length $\eta n$. Transmitter 2 produces a sequence of equal length by encoding Message $M_{2}$ with power $P_{2}$ using the concatenated scheme and sends this sequence.

We next present a rough overview of the decoding at the receiver. The receiver first decodes the pair $\left(M_{1, \mathrm{CS}}, M_{2}\right)$ by using the inner and the outer decoder of our concatenated scheme and treating the transmission of Message $M_{1, \mathrm{NF}}$ as additional noise. From its guess of $\left(M_{1, \mathrm{NF}}, M_{2}\right)$ the receiver cannot recover the sequences produced by our concatenated scheme because it is incognizant of the feedback noise. Nevertheless, it can form an estimate of both produced sequences (pretending that its guess of $\left(M_{1, \mathrm{CS}}, M_{2}\right)$ is correct) and subtract the sum of the estimates from the received signal. Based on the resulting difference the receiver finally decodes message $M_{1, \mathrm{NF}}$, which concludes the decoding.

In the following we describe the scheme in more detail. Given $M_{1, \mathrm{NF}}=m_{1, \mathrm{NF}}$, Transmitter 1 picks the codeword $\mathbf{u}\left(m_{1, \mathrm{NF}}\right) \triangleq\left(u_{1}, \ldots, u_{\eta n}\right)^{\top}$ corresponding to $m_{1, \mathrm{NF}}$ from its Gaussian codebook. Given $M_{1, \mathrm{CS}}=m_{1, \mathrm{CS}}$, Transmitter 1 feeds $m_{1, \mathrm{CS}}$ to Outer Encoder 1, which picks the codeword $\boldsymbol{\xi}_{1}\left(m_{1, \mathrm{CS}}\right) \triangleq\left(\xi_{1,1}, \ldots, \xi_{1, n}\right)^{\mathrm{\top}}$ corresponding to $m_{1, \mathrm{CS}}$ from its codebook and feeds it to Inner Encoder 1. Similarly, given $M_{2}=m_{2}$, Transmitter 2 feeds $m_{2}$ to Outer Encoder 2, which picks the codeword $\boldsymbol{\xi}_{2}\left(m_{2}\right) \triangleq\left(\xi_{2,1}, \ldots, \xi_{2, n}\right)^{\top}$ corresponding to $m_{2}$ and feeds it to Inner Encoder 2. Denoting the parameters of the inner encoders by $\mathbf{a}_{1}, \mathbf{a}_{2}, \mathrm{~B}_{1}$, and $\mathrm{B}_{2}$, respectively, Inner Encoder 1 forms the $\eta$-dimensional vectors

$$
\mathbf{a}_{1} \xi_{1, k}+\mathrm{B}_{1} \mathbf{V}_{1, k}, \quad k \in\{1, \ldots, n\}
$$

and Inner Encoder 2 forms the $\eta$-dimensional vectors

$$
\mathbf{a}_{2} \xi_{2, k}+\mathrm{B}_{2} \mathbf{V}_{2, k}, \quad k \in\{1, \ldots, n\}
$$

where for $\nu \in\{1,2\}$ :

$$
\mathbf{V}_{\nu, k} \triangleq\left(V_{\nu,(k-1) \eta+1}, \ldots, V_{\nu, k \eta}\right)^{\top} \text {. }
$$

\footnotetext{
${ }^{9}$ To satisfy the powers constraints the Gaussian codewords should be of variance slightly less than $P_{1}^{\prime}$. However, this is a technicality which we ignore.
}

The signal transmitted by Transmitter 1 is the sum of the vectors in (46) and the vectors

$$
\mathbf{u}_{k} \triangleq\left(u_{(k-1) \eta+1}, \ldots, u_{k \eta}\right)^{\top}, \quad k \in\{1, \ldots, n\}
$$

i.e.,

$$
\mathbf{X}_{1, k}=\mathbf{u}_{k}+\mathbf{a}_{1} \xi_{1, k}+\mathrm{B}_{1} \mathbf{V}_{1, k}, \quad k \in\{1, \ldots, n\}
$$

where

$$
\mathbf{X}_{1, k} \triangleq\left(X_{1,(k-1) \eta+1}, \ldots, X_{1, k \eta}\right)^{\top}, \quad k \in\{1, \ldots, n\} .
$$

The signal transmitted by Transmitter 2 is described by the vectors in (47), as follows:

$$
\mathbf{X}_{2, k}=\mathbf{a}_{2} \xi_{2, k}+\mathrm{B}_{2} \mathbf{V}_{2, k}, \quad k \in\{1, \ldots, n\}
$$

where

$$
\mathbf{X}_{2, k} \triangleq\left(X_{2,(k-1) \eta+1}, \ldots, X_{2, k \eta}\right)^{\top} \text {. }
$$

Notice that if $\mathbf{a}_{1}, \mathbf{a}_{2}, \mathrm{~B}_{1}, \mathrm{~B}_{2}$ satisfy (19) for powers $\left(P_{1}-P_{1}^{\prime}\right)$ and $P_{2}$, noise variance $\left(N+P_{1}^{\prime}\right)$ and feedback-noise covariance matrix $\mathrm{K}_{W_{1} W_{2}}$ and if the outer code's codewords are zero-mean and average block-power constrained to 1 , then for sufficiently large blocklength $n$ the input sequences (48) and (49) satisfy the power constraint (4) with arbitrary high probability.

We next describe the decoding. The receiver first decodes the pair $\left(M_{1, \mathrm{CS}}, M_{2}\right)$ based on the tuple $\left(Y_{1}, \ldots Y_{\eta n}\right)$ by treating the codeword $\mathbf{U}\left(M_{1, \mathrm{NF}}\right)$ as additional noise and by applying the inner and outer decoders of the concatenated scheme. Let $\hat{M}_{\text {ICS }}$ and $\hat{M}_{2}$ denote the receiver's guesses of the messages $M_{\text {ICS }}$ and $M_{2}$, and let $\left(\hat{\Xi}_{1,1}^{(\mathrm{Rx})}, \ldots, \hat{\Xi}_{1, n}^{(\mathrm{Rx})}\right)$ and $\left(\hat{\Xi}_{2,1}^{(\mathrm{Rx})}, \ldots, \hat{\Xi}_{2, n}^{(\mathrm{Rx})}\right)$ denote the corresponding codewords in the outer codes. The receiver then attempts to estimate and subtract the influence of the concatenated scheme (see (46) and(47)) by computing for each $k \in\{1, \ldots, n\}$ the difference

$$
\tilde{\mathbf{Y}}_{k} \triangleq\left(\mathrm{I}_{\eta}-\mathrm{B}_{1}-\mathrm{B}_{2}\right) \mathbf{Y}_{k}-\mathbf{a}_{1} \hat{\Xi}_{1, k}^{(\mathrm{Rx})}-\mathbf{a}_{2} \hat{\Xi}_{2, k}^{(\mathrm{Rx})}
$$

where the $\eta$-dimensional vector $\mathbf{Y}_{k}$ is defined as

$$
\mathbf{Y}_{k} \triangleq\left(Y_{(k-1) \eta+1}, \ldots, Y_{k \eta}\right)^{\top} \text {. }
$$

If the receiver decoded $M_{1, \mathrm{CS}}$ and $M_{2}$ correctly, i.e., if $\hat{M}_{1, \mathrm{CS}}=$ $M_{1, \mathrm{CS}}$ and $\hat{M}_{2}=M_{2}$, then (50) corresponds to

$$
\mathbf{U}_{k}+\mathrm{B}_{1} \mathbf{W}_{1, k}+\mathrm{B}_{2} \mathbf{W}_{2, k}+\mathbf{Z}_{k}, \quad k \in\{1, \ldots, n\} .
$$

Finally, the receiver decodes Message $M_{1, \mathrm{NF}}$ based on the differences $\left\{\tilde{\mathbf{Y}}_{i}\right\}_{i=1}^{n}$ using an optimal decoder for a Gaussian $\eta$-input antenna $/ \eta$-output antenna channel where the noise sequences are white but correlated across antennas. Notice that because of the correlation of the noise sequences across antennas, the scheme might be improved if correlated Gaussian codewords are used to transmit Message $M_{1, \mathrm{NF}}$.

2) Rate-Splitting With Carleial's Scheme: Our second extension is based on modifying Carleial's rate-splitting scheme [2]. Carleial's scheme combines a variation of the Cover-Leung scheme [4] with a no-feedback scheme by means of rate-splitting. Here, we propose to modify his scheme by replacing the 
no-feedback scheme with our concatenated scheme. Since for $\eta=1, a_{1}=\sqrt{P_{1}}$, and $a_{2}=\sqrt{P_{2}}$ our concatenated scheme results in an optimal no-feedback scheme, our proposed extension includes Carleial's scheme as a special case. In the following we roughly sketch the idea behind our extended scheme. For more details see Appendix H.

Our scheme is a Block-Markov scheme of blocklength $n^{\prime}$. Each block of $n^{\prime}$ channel uses is divided into $(B+1)$ blocks, each of length $\eta n$ for positive integers $\eta$ and $n$, i.e., we assume that $n^{\prime}=(B+1) \eta n$. Each transmitter splits its message into two sequences of independent submessages: Transmitter $\nu$, for $\nu \in\{1,2\}$, splits its message $M_{\nu}$ into a sequence of independent submessages $\left\{M_{\nu, \mathrm{CL}, 1}, \ldots, M_{\nu, \mathrm{CL}, B}\right\}$ of rates $R_{\nu, \mathrm{CL}}$ and into a sequence of independent submessages $\left\{M_{\nu, \mathrm{CS}, 1}, \ldots, M_{\nu, \mathrm{CS}, B}\right\}$ of rates $R_{\nu, \mathrm{CS}}$. The rates $R_{\nu, \mathrm{CL}}$ and $R_{\nu, \mathrm{CS}}$ should be nonnegative and sum to $R_{\nu} \frac{B+1}{B}$, but otherwise can be chosen arbitrary depending on the parameters of the setting. Similarly, for Transmitter 2. (Here, the subscript CL stands for "Cover-Leung" and the subscript CS stands for "concatenated scheme.")

As in Carleial's scheme, after each block $b \in\{1, \ldots, B\}$ Transmitter 1 and Transmitter 2 decode the other transmitter's submessage $M_{2, \mathrm{CL}, b}$ and $M_{1, \mathrm{CL}, b}$ based on their feedback outputs. The two transmitters can accomplish the decodings in two different ways. Transmitter 1 either directly decodes Message $M_{2, \mathrm{CL}, b}$, or it first decodes $M_{2, \mathrm{CS}, b}$ before decoding the desired message $M_{2, \mathrm{CL}, b}$. Which alternative is better depends on the specific parameters of the setting.

The encoding is performed as follows. To encode messages $\left\{M_{\nu, \mathrm{CL}, b}\right\}_{b=1}^{B}$ Transmitter $\nu$, for $\nu \in\{1,2\}$, uses Carleial's variation of the Cover-Leung scheme and to encode messages $\left\{M_{\nu, \mathrm{CS}, b}\right\}_{b=1}^{B}$ it uses our concatenated scheme. More specifically, before the transmission in Block $b \in\{1, \ldots, B\}$ starts, Transmitter $\nu$ chooses the codewords for messages $M_{\nu, \mathrm{CL}, b}$, $M_{1, \mathrm{CL}, b-1}$, and $M_{2, \mathrm{CL}, b-1}$ from the corresponding Gaussian codebooks and produces an $\eta n$-length sequence of power $P_{\nu}^{\prime}$, for some $0 \leq P_{\nu}^{\prime} \leq P_{\nu}$, by taking a linear combination of the chosen codewords. It also produces an $\eta n$-length sequence of power $\left(P_{\nu}-P_{\nu}^{\prime}\right)$ by encoding message $M_{\nu, \mathrm{CS}, b}$ using the outer and inner encoders of our concatenated scheme where $\eta$ is the parameter of the inner code and $n$ is the blocklength of the outer code. It sends the sum of the two produced sequences in Block $b$. In Block $(B+1)$ Transmitter $\nu$ picks the codewords for messages $M_{1, \mathrm{CL}, B}$ and $M_{2, \mathrm{CL}, B}$ from the corresponding Gaussian codebooks and sends a linear combination of power $P_{\nu}^{\prime}$ of these codewords.

After each Block $b \in\{1, \ldots, B\}$ the receiver decodes messages $M_{1, \mathrm{CS}, b}, M_{2, \mathrm{CS}, b} M_{1, \mathrm{CL}, b-1}$, and $M_{2, \mathrm{CL}, b-1}$. It first decodes messages $M_{1, \mathrm{CS}, b}$ and $M_{2, \mathrm{CS}, b}$ using inner and outer decoder of our concatenated scheme and treating the sequences produced by encoding messages $M_{1, \mathrm{CL}, b-1}, M_{2, \mathrm{CL}, b-1}$, $M_{1, \mathrm{CL}, b}$ and $M_{2, \mathrm{CL}, b}$ as additional noise. From its guess of $\left(M_{1, \mathrm{CS}, b}, M_{2, \mathrm{CS}, b}\right)$ the receiver cannot recover the sequences produced by our concatenated scheme because it is incognizant of the feedback noise. Nevertheless, it can form an estimate of both produced sequences (pretending that its guess is correct) and subtract the sum of the estimates from the received signal. Based on the resulting difference and based on similar differ- ences which resulted in the previous block, it then decodes messages $\left(M_{1, \mathrm{CL}, b-1}, M_{2, \mathrm{CL}, b-1}\right)$. After the last block $(B+1)$ the receiver decodes the pair $\left(M_{1, \mathrm{CL}, B}, M_{2, \mathrm{CL}, B}\right)$. More general decoding orders at the receiver could be considered, but for simplicity, we restrict attention to this order.

3) Interleaving and Rate-Splitting With Carleial's Scheme: Our third extension is based on rate-splitting an interleaved version of Carleial's Cover-Leung scheme with an interleaved version of our concatenated scheme. We only describe here the general structure of the scheme. For more details see Appendix I.

Our scheme is a Block-Markov scheme of blocklenght $n^{\prime}$. Each block of $n^{\prime}$ channel uses is divided into $(B+1)$ blocks, each of length $\eta n$ and each such block is further divided into $\eta$ subblocks of length $n$. Thus, it is assumed that $B, \eta$, and $n$ are positive integers such that $n^{\prime}=(B+1) \eta n$. Similarly, each transmitter splits its message into two sequences of independent submessages: Transmitter $\nu$, for $\nu \in\{1,2\}$, splits its message $M_{\nu}$ into a sequence of independent submessages $\left\{M_{\nu, \mathrm{ICL}, 1}, \ldots, M_{\nu, \mathrm{ICL}, \eta B}\right\}$ and into a sequence of independent submessages $\left\{M_{\nu, \mathrm{ICS}, 1}, \ldots, M_{\nu, \mathrm{ICS}, B}\right\}$. Notice that the first sequence of submessages is of length $\eta B$, and the second of length $B$. Messages $\left\{M_{\nu, \mathrm{ICL},(b-1) \eta+\ell}\right\}_{b=1}^{B}$ are of rate $R_{\nu, \mathrm{ICL}, \ell}$, and Messages $\left\{M_{\nu, \mathrm{ICS}, b}\right\}_{b=1}^{B}$ of rate $R_{1, \mathrm{ICS}}$. The rates $R_{\nu, \mathrm{ICL}, 1}, \ldots, R_{\nu, \mathrm{ICL}, \eta}$, and $R_{\nu, \mathrm{ICS}}$ should be nonnegative and sum to $R_{\nu} \frac{B+1}{B}$, but otherwise can be chosen arbitrary depending on the parameters of the setting. (The subscript ICL stands for "interleaved Cover-Leung" and the subscript ICS stands for "interleaved concatenated scheme.")

Similar to the previous extension and similar to Carleial's scheme, the transmitters decode part of the other transmitter's messages based on their feedback outputs. Specifically in this scheme, after each subblock $\tilde{b} \in\{1, \ldots, B \eta\}$, Transmitters 1 and 2 decode the other transmitter's submessage $M_{2, \mathrm{ICL}, \tilde{b}}$ and $M_{1, \mathrm{ICL}, \tilde{b}}$. Following this decoding step, the transmitters compute "cleaned" feedback outputs, i.e., they mitigate the influence of the Cover-Leung messages $M_{1, \mathrm{ICL}, \tilde{b}}, M_{2, \mathrm{ICL}, \tilde{b}}, M_{1, \mathrm{ICL}, \tilde{b}-\eta}$, and $M_{2, \mathrm{ICL}, \tilde{b}-\eta}$ transmitted in this block on the observed feedback outputs. Transmitter 1 computes its "cleaned" feedback output more specifically as follows. It first reconstructs the sequence that was produced by Transmitter 2 in this subblock $\tilde{b}$ to encode messages $M_{2, \mathrm{ICL}, \tilde{b}}, M_{1, \mathrm{ICL}, \tilde{b}-\eta}$, and $M_{2, \mathrm{ICL}, \tilde{b}-\eta}$ (pretending that its guesses of $M_{2, \mathrm{ICL}, \tilde{b}}$ and $M_{2, \mathrm{ICL}, \tilde{b}-\eta}$ are correct). It then subtracts this reconstructed sequence and the sequence it pro-

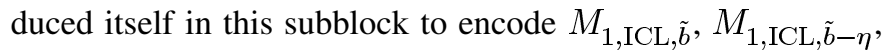
and $M_{2, \mathrm{ICL}, \tilde{b}-\eta}$ from its observed feedback outputs. Similarly for Transmitter 2

The encoding is performed as follows. To encode Messages $\left\{M_{\nu, \mathrm{ICL}, k}\right\}_{k=1}^{\eta B}$, Transmitter $\nu$, for $\nu \in\{1,2\}$, uses an interleaved version of Carleial's Cover-Leung scheme, and to encode Messages $\left\{M_{\nu, \mathrm{ICS}, b}\right\}_{b=1}^{B}$ it uses an interleaved version of our concatenated scheme. We describe these encodings in more detail. In a fixed block $b \in\{1, \ldots, B\}$, Transmitter $\nu$ sends the sum of two $\eta n$-length sequences. The first sequence is of power $P_{\nu}^{\prime}$, for some $0 \leq P_{\nu}^{\prime} \leq P_{\nu}$, and consists of $\eta$ subblocks. The $\ell$ th subblock of the sequence, for $\ell \in\{1, \ldots, \eta\}$, Transmitter $\nu$ chooses the $n$-length codewords for Messages $M_{\nu, \mathrm{ICL},(b-1) \eta+\ell}, M_{1, \mathrm{ICL},(b-2) \eta+\ell}$, and $M_{2, \mathrm{ICL},(b-2) \eta+\ell}$ from 
the corresponding Gaussian codebooks and takes a linear combination of these chosen codewords. We notice that here each pair of messages $\left(M_{1, \mathrm{ICL}, \tilde{b}}, M_{2, \mathrm{ICL}, \tilde{b}}\right)$, for $\tilde{b} \in\{1, \ldots, B \eta\}$, is encoded into Subblocks $\tilde{b}$ and $\tilde{b}+\eta$, and not-as in Carleial's original scheme-into Subblocks $\tilde{b}$ and $\tilde{b}+1$. The second sequence is of power $\left(P_{1}-P_{1}^{\prime}\right)$ and produced as follows: Transmitter $\nu$ first applies its outer encoder to encode Message $M_{\nu, \mathrm{ICS}, b}$, and then feeds the outcome to a modified version of its inner encoder. The inner encoder is modified as described by the following two items. 1) Instead of the original feedback the modified inner encoder uses the "cleaned" feedback mentioned above, where the influence of the interleaved Cover-Leung type scheme is mitigated. 2) Unlike the original inner encoder where the $\ell$ th fed codeword symbol is encoded into $\eta$ subsequent symbols at positions $(\ell-1) \eta+1$ to $\ell \eta$, the modified inner encoder encodes the $\ell$ th fed codeword symbol into the $\eta$ symbols at positions $\ell, n+\ell, \ldots,(\eta-1) n+\ell$, for $\ell \in\{1, \ldots, \eta\}$.

Notice that the chosen interleaving of the modified inner encoders preserves the causality of the feedback. Moreover, it implies that in the interleaved sequence the symbols in Subblock $\tilde{b}$, for $\tilde{b} \in\{(b-1) \eta+1, \ldots, b \eta\}$, only depend on feedback outputs of previous subblocks $1, \ldots, \tilde{b}-1$ and not on feedback outputs of the current Subblock $\tilde{b}$. This is the reason why the modified inner encoder can use the "cleaned" feedback instead of the original feedback.

The receiver first decodes Messages $\left\{M_{1, \mathrm{ICL}, \tilde{b}}\right\}_{\tilde{b}=1}^{\eta B}$ and $\left\{M_{2, \mathrm{ICL}, \tilde{b}}\right\}_{\tilde{b}=1}^{\eta B}$ and only thereafter decodes Messages $\quad\left\{M_{1, \mathrm{ICS}, b}\right\}_{b=1}^{B} \quad$ and $\quad\left\{M_{2, \mathrm{ICS}, b}\right\}_{b=1}^{B}$. More specifically, the receiver first decodes Messages $\left\{\left(M_{1, \mathrm{ICL},(b-1) \eta+1}, M_{2, \mathrm{ICL},(b-1) \eta+1}\right)\right\}_{b=1}^{B}$, followed by Messages $\left\{\left(M_{1, \mathrm{ICL},(b-1) \eta+2}, M_{2, \mathrm{ICL},(b-1) \eta+2}\right)\right\}_{b=1}^{B}$, etc. The receiver then reconstructs the sequences produced to encode these messages (pretending its guesses are correct) and subtracts them from the received signal. Based on the resulting difference, which we call the "cleaned" output signal, the receiver decodes Messages $\left\{M_{1, \mathrm{ICS}, b}\right\}_{b=1}^{B}$ and $\left\{M_{2, \mathrm{ICS}, b}\right\}_{b=1}^{B}$. To this end, it first reverses the interleaving and then applies the inner and outer decoders of our concatenated scheme.

Notice that in the presented scheme, Messages $\left\{M_{1, \mathrm{ICS}, b}\right\}_{b=1}^{B}$ and $\left\{M_{2, \mathrm{ICS}, b}\right\}_{b=1}^{B}$ are decoded based on the "cleaned" output signal and they are encoded using the "cleaned" feedbacks. The "cleaned" output signal and the "cleaned" feedbacks correspond to the output signals and the feedbacks in a situation where only the interleaved concatenated scheme is employed but not the interleaved version of Carleial's Cover-Leung scheme. Therefore, in the presented rate-splitting scheme there is no degradation in performance of the interleaved concatenated scheme due to the rate-splitting with Carleial's Cover-Leung scheme.

Further, notice that in a given Block $b \in\{1, \ldots, B\}$ the sum of the two sequences produced to encode Messages $M_{1, \mathrm{ICS}, b}$ and $M_{2, \mathrm{ICS}, b}$ is of different power in each of the $\eta$ subblocks. Thus, these sequences introduce different noise levels on the receiver's decoding of Messages $\left\{\left(M_{1, \mathrm{ICL},(b-1) \eta+\ell}, M_{2, \mathrm{ICL},(b-1) \eta+\ell)}\right\}_{\ell=1}^{\eta}\right.$, and consequently the rates $\left\{R_{1, \mathrm{ICL}, \ell}\right\}_{\ell=1}^{\eta}$ and $\left\{R_{2, \mathrm{ICL}, \ell}\right\}_{\ell=1}^{\eta}$ should be chosen depending on $\ell$.

\section{E. Proofs}

1) Proof of Proposition IV.5: We first prove Part 1). To this end, we show that for every fixed $\eta \in \mathbb{N}$ and fixed $\eta$-dimensional vectors $\mathbf{a}_{1}, \mathbf{a}_{2}, \eta \times \eta$-dimensional matrices $\mathrm{B}_{1}, \mathrm{~B}_{2}$, and $2 \times$ $\eta$-dimensional matrix $C$, the following two statements hold.

i) For all positive semidefinite matrices $\mathrm{K}_{W_{1} W_{2}}$ and $\mathrm{K}_{W_{1} W_{2}}^{\prime}$ :

$$
\begin{aligned}
& \left(\mathrm{K}_{W_{1} W_{2}} \succeq \mathrm{K}_{W_{1} W_{2}}^{\prime}\right) \Longrightarrow \\
& \left(\mathcal{R}\left(N, \mathrm{~K}_{W_{1} W_{2}} ; \eta, \mathbf{a}_{1}, \mathbf{a}_{2}, \mathrm{~B}_{1}, \mathrm{~B}_{2}, \mathrm{C}\right)\right. \\
& \left.\quad \subseteq \mathcal{R}\left(N, \mathrm{~K}_{W_{1} W_{2}}^{\prime} ; \eta, \mathbf{a}_{1}, \mathbf{a}_{2}, \mathrm{~B}_{1}, \mathrm{~B}_{2}, \mathrm{C}\right)\right) .
\end{aligned}
$$

ii) If the choice of parameters $\eta, \mathbf{a}_{1}, \mathbf{a}_{2}, \mathrm{~B}_{1}, \mathrm{~B}_{2}, \mathrm{C}$ satisfies the power constraints (19) for a covariance matrix $\mathrm{K}_{W_{1} W_{2}}$, then it also satisfies these power constraints for all covariance matrices $\mathrm{K}_{W_{1} W_{2}}^{\prime}$ for which $\mathrm{K}_{W_{1} W_{2}} \succeq$ $\mathrm{K}_{W_{1} W_{2}}^{\prime}$.

By Definition IV.2, Statements i) and ii) imply that

$$
\begin{aligned}
\left(\mathrm{K}_{W_{1} W_{2}} \succeq \mathrm{K}_{W_{1} W_{2}}^{\prime}\right) \Longrightarrow \\
\left(\mathcal{R}\left(P_{1}, P_{2}, N, \mathrm{~K}_{W_{1} W_{2}}\right) \subseteq \mathcal{R}\left(P_{1}, P_{2}, N, \mathrm{~K}_{W_{1} W_{2}}^{\prime}\right)\right),
\end{aligned}
$$

and thus conclude the proof of Part 1).

We start by proving Statement i). Fix a tuple $\left(\eta, \mathbf{a}_{1}, \mathbf{a}_{2}, \mathrm{~B}_{1}, \mathrm{~B}_{2}, \mathrm{C}\right)$. We only prove Statement i) for the case where $C C^{\top}$ is nonsingular. For the case where $C C^{\top}$ is singular but $C \neq 0$ the proof is analogous and therefore omitted; for $C=0$ the proof is trivial. To establish Statement i) when $\mathrm{CC}^{\top}$ is nonsingular, it suffices to show that all three RHSs of (18) are monotonically decreasing in $\mathrm{K}_{W_{1} W_{2}}$ with respect to the Loewner order. We only prove the monotonicity of the RHS of (18a); the monotonicities of the RHSs of (18b) and $(18 \mathrm{c})$ can be shown analogously. Thus, in the following we fix two positive semidefinite $2 \times 2$ matrices $\mathrm{K}_{W_{1} W_{2}}$ and $\mathrm{K}_{W_{1} W_{2}}^{\prime}$ satisfying $\mathrm{K}_{W_{1} W_{2}} \succeq \mathrm{K}_{W_{1} W_{2}}^{\prime}$ and we show that:

$$
\begin{aligned}
& \frac{1}{2 \eta} \log \left(\frac{\left|\mathrm{C}\left(\mathbf{a}_{1} \mathbf{a}_{1}^{\top}+N \mathrm{I}_{\eta}+\mathrm{B}_{\mathrm{r}}\left(\mathrm{K}_{W_{1} W_{2}} \otimes \mathrm{I}_{\eta}\right) \mathrm{B}_{\mathrm{r}}^{\top}\right) \mathrm{C}^{\top}\right|}{\left|\mathrm{C}\left(N \mathrm{I}_{\eta}+\mathrm{B}_{\mathrm{r}}\left(\mathrm{K}_{W_{1} W_{2}} \otimes \mathrm{I}_{\eta}\right) \mathrm{B}_{\mathrm{r}}^{\top}\right) \mathrm{C}^{\top}\right|}\right) \\
& \geq \frac{1}{2 \eta} \log \left(\frac{\left|\mathrm{C}\left(\mathbf{a}_{1} \mathbf{a}_{1}^{\top}+N \mathrm{I}_{\eta}+\mathrm{B}_{\mathrm{r}}\left(\mathrm{K}_{W_{1} W_{2}}^{\prime} \otimes \mathrm{I}_{\eta}\right) \mathrm{B}_{\mathrm{r}}^{\top}\right) \mathrm{C}^{\top}\right|}{\left|\mathrm{C}\left(N \mathrm{I}_{\eta}+\mathrm{B}_{\mathrm{r}}\left(\mathrm{K}_{W_{1} W_{2}}^{\prime} \otimes \mathrm{I}_{\eta}\right) \mathrm{B}_{\mathrm{r}}^{\top}\right) \mathrm{C}^{\top}\right|}\right) .
\end{aligned}
$$

Before proving (51) we recall the following well-known properties of positive semidefinite matrices. For all positive semidefinite $n \times n$ matrices $\mathrm{K}, \mathrm{K}_{1}, \mathrm{~K}_{2}$ satisfying $\mathrm{K}_{1} \succeq \mathrm{K}_{2}$ and for all $m \times n$ matrices $\mathrm{M}$ the following properties hold:

$$
\begin{aligned}
\mathrm{MK}_{1} \mathrm{M}^{\top} & \succeq \mathrm{MK}_{2} \mathrm{M}^{\top}, \\
\mathrm{K}+\mathrm{K}_{1} & \succeq \mathrm{K}+\mathrm{K}_{2}, \\
\mathrm{KK}_{1} & \succeq \mathrm{KK}_{2}, \\
\mathrm{~K}_{1}^{-1} & \preceq \mathrm{K}_{2}^{-1},
\end{aligned}
$$

and

$$
\begin{gathered}
\left|\mathrm{K}_{1}\right| \geq\left|\mathrm{K}_{2}\right|, \\
\operatorname{tr}\left(\mathrm{K}_{1}\right) \geq \operatorname{tr}\left(\mathrm{K}_{2}\right) .
\end{gathered}
$$


Based on these properties and the definition $A_{1} \triangleq \mathbf{a}_{1} \mathbf{a}_{1}^{\top}$ the following sequence of implications can be proved:

$$
\begin{aligned}
& \left(\mathrm{K}_{W_{1} W_{2}} \succeq \mathrm{K}_{W_{1} W_{2}}^{\prime}\right) \\
& \Longrightarrow\left(\left(\mathrm{K}_{W_{1} W_{2}} \otimes \mathrm{I}_{\eta}\right) \succeq\left(\mathrm{K}_{W_{1} W_{2}}^{\prime} \otimes \mathrm{I}_{\eta}\right)\right) \\
& \Longrightarrow\left(\left(\mathrm{B}_{\mathrm{r}}\left(\mathrm{K}_{W_{1} W_{2}} \otimes \mathrm{I}_{\eta}\right) \mathrm{B}_{\mathrm{r}}^{\top}\right) \succeq\left(\mathrm{B}_{\mathrm{r}}\left(\mathrm{K}_{W_{1} W_{2}}^{\prime} \otimes \mathrm{I}_{\eta}\right) \mathrm{B}_{\mathrm{r}}^{\top}\right)\right) \\
& \Longrightarrow\left(\left(N \mathrm{I}_{\eta}+\mathrm{B}_{\mathrm{r}}\left(\mathrm{K}_{W_{1} W_{2}} \otimes \mathrm{I}_{\eta}\right) \mathrm{B}_{\mathrm{r}}^{\top}\right)\right. \\
& \left.\succeq\left(N \mathrm{I}_{\eta}+\mathrm{B}_{\mathrm{r}}\left(\mathrm{K}_{W_{1} W_{2}}^{\prime} \otimes \mathrm{I}_{\eta}\right) \mathrm{B}_{\mathrm{r}}^{\mathrm{T}}\right)\right) \\
& \Longrightarrow\left(\left(\mathrm{C}\left(N \mathrm{I}_{\eta}+\mathrm{B}_{\mathrm{r}}\left(\mathrm{K}_{W_{1} W_{2}} \otimes \mathrm{I}_{\eta}\right) \mathrm{B}_{\mathrm{r}}^{\top}\right) \mathrm{C}^{\top}\right)\right. \\
& \left.\succeq\left(\mathrm{C}\left(N \mathrm{I}_{\eta}+\mathrm{B}_{\mathrm{r}}\left(\mathrm{K}_{W_{1} W_{2}}^{\prime} \otimes \mathrm{I}_{\eta}\right) \mathrm{B}_{\mathrm{r}}^{\mathrm{T}}\right) \mathrm{C}^{\mathrm{T}}\right)\right) \\
& \Longrightarrow\left(\left(\mathrm{C}\left(N \mathrm{I}_{\eta}+\mathrm{B}_{\mathrm{r}}\left(\mathrm{K}_{W_{1} W_{2}} \otimes \mathrm{I}_{\eta}\right) \mathrm{B}_{\mathrm{r}}^{\top}\right) \mathrm{C}^{\top}\right)^{-1}\right. \\
& \left.\preceq\left(\mathrm{C}\left(N \mathrm{I}_{\eta}+\mathrm{B}_{\mathrm{r}}\left(\mathrm{K}_{W_{1} W_{2}}^{\prime} \otimes \mathrm{I}_{\eta}\right) \mathrm{B}_{\mathrm{r}}^{\top}\right) \mathrm{C}^{\top}\right)^{-1}\right) \\
& \left.\overrightarrow{\left(\left(\mathrm{I}_{2}\right.\right.}+\mathrm{CA}_{1} \mathrm{C}^{\top}\left(\mathrm{C}\left(N \mathrm{I}_{\eta}+\mathrm{B}_{\mathrm{r}}\left(\mathrm{K}_{W_{1} W_{2}} \otimes \mathrm{I}_{\eta}\right) \mathrm{B}_{\mathrm{r}}^{\top}\right) \mathrm{C}^{\top}\right)^{-1}\right) \\
& \left.\preceq\left(\mathrm{I}_{2}+\mathrm{CA}_{1} \mathrm{C}^{\top}\left(\mathrm{C}\left(N \mathrm{I}_{\eta}+\mathrm{B}_{\mathrm{r}}\left(\mathrm{K}_{W_{1} W_{2}}^{\prime} \otimes \mathrm{I}_{\eta}\right) \mathrm{B}_{\mathrm{r}}^{\top}\right) \mathrm{C}^{\top}\right)^{-1}\right)\right)
\end{aligned}
$$

where (58) follows by the linearity of the Kronecker product $\otimes$ and because for every positive semidefinite matrix $\mathrm{K}$ also the Kronecker product $\mathrm{K} \otimes \mathrm{I}_{\eta}$ is positive semidefinite ${ }^{10}$; where (58) follows by (52); where (60) follows by (53) and because $N \mathrm{I}_{\eta} \succeq 0$; where (61) follows by (52); where (62) follows by (55); where (63) follows by (54) and (55) and because $A_{1} \succeq 0$, and thus, by (52), also $\mathrm{CA}_{1} \mathrm{C}^{\top} \succeq 0$.

Inequality (51) follows then from (63), from (56), from the monotonicity of the log-function, and from the fact that for every $2 \times 2$ positive semidefinite matrix $\mathrm{K}$, for $\mathrm{A}_{1}$ as defined above, and when $\mathrm{CC}^{\top}$ is nonsingular:

$$
\begin{aligned}
& \frac{1}{2 \eta} \log \left(\frac{\left|\mathrm{C}\left(\mathbf{a}_{1} \mathbf{a}_{1}^{\top}+N \mathrm{I}_{\eta}+\mathrm{B}_{\mathrm{r}}\left(\mathrm{K} \otimes \mathrm{I}_{\eta}\right) \mathrm{B}_{\mathrm{r}}^{\top}\right) \mathrm{C}^{\top}\right|}{\left|\mathrm{C}\left(N \mathrm{I}_{\eta}+\mathrm{B}_{\mathrm{r}}\left(\mathrm{K} \otimes \mathrm{I}_{\eta}\right) \mathrm{B}_{\mathrm{r}}^{\top}\right) \mathrm{C}^{\top}\right|}\right) \\
& =\frac{1}{2 \eta} \log \left(\mid \mathrm{I}_{2}+\mathrm{CA}_{1} \mathrm{C}^{\top}\right. \\
& \left.\cdot\left(\mathrm{C}\left(N \mathrm{I}_{\eta}+\mathrm{B}_{\mathrm{r}}\left(\mathrm{K}_{W_{1} W_{2}}^{\prime} \otimes \mathrm{I}_{\eta}\right) \mathrm{B}_{\mathrm{r}}^{\top}\right) \mathrm{C}^{\top}\right)^{-1} \mid\right)
\end{aligned}
$$

which holds because for all nonsingular square matrices $M_{1}$ and $\mathrm{M}_{2}$ of the same dimension $\frac{\left|\mathrm{M}_{1}\right|}{\left|\mathrm{M}_{2}\right|}=\left|\mathrm{M}_{1} \mathrm{M}_{2}^{-1}\right|$. This concludes the proof of Statement i).

We next prove Statement ii). It suffices to show that for fixed parameters $\eta, \mathbf{a}_{2}, \mathbf{a}_{2}, \mathrm{~B}_{1}, \mathrm{~B}_{2}, \mathrm{C}$, the left-hand sides of the power constraints (19) are monotonically increasing in $\mathrm{K}_{W_{1} W_{2}}$ with respect to the Loewner order. Similarly to the proof of Statement i), this can be shown by a sequence of implications based on (52), on (53), on (57), on the fact that $\mathrm{K}_{W_{1} W_{2}} \succeq \mathrm{K}_{W_{1} W_{2}}^{\prime}$ implies

\footnotetext{
${ }^{10}$ That $\mathbf{K} \succeq 0$ implies $(\mathbf{K} \otimes \mathbf{I}) \succeq 0$ can be seen as follows. For every $2 \eta$-dimensional vector $\mathbf{x} \triangleq\left(x_{1}, \ldots, x_{2 \eta}\right)^{\top}$, where we define $\mathbf{x}_{i} \triangleq\left(x_{2 i-1}, x_{2 i}\right)^{\top}$ for $i \in\{1, \ldots, \eta\}$, and every $2 \times 2$ positive semidefinite matrix $\mathrm{K}$ the term $\mathbf{x}^{\top}\left(\mathbf{K} \otimes \mathbf{I}_{\eta}\right) \mathbf{x}$ can be written as $\sum_{i=1}^{\eta} \mathbf{x}_{i}^{\top} \mathbf{K} \mathbf{x}_{i}$, which is nonnegative since $\mathrm{K}$ is positive semidefinite.
}

$\left(\mathrm{K}_{W_{1} W_{2}} \otimes \mathrm{I}_{\eta}\right) \succeq\left(\mathrm{K}_{W_{1} W_{2}}^{\prime} \otimes \mathrm{I}_{\eta}\right)$, and on the fact that the trace of a sum equals the sum of the traces. The details are omitted.

We prove Part 2). The inclusion of the LHS in the RHS is trivial, because for every positive $\delta$ all choices of parameters $\eta, \mathbf{a}_{1}, \mathbf{a}_{2}, \mathrm{~B}_{1}, \mathrm{~B}_{2}, \mathrm{C}$ satisfying the power constraints (19) for powers $\left(P_{1}-\delta\right)$ and $\left(P_{2}-\delta\right)$ satisfy the power constraints also for powers $P_{1}$ and $P_{2}$.

The inclusion of the RHS in the LHS is proved as follows. We fix a rate pair $\left(R_{1}^{\circ}, R_{2}^{\circ}\right)$ in the interior of $\mathcal{R}\left(P_{1}, P_{2}, N, \mathrm{~K}_{W_{1} W_{2}}\right)$, i.e.,

$$
\left(R_{1}^{\circ}, R_{2}^{\circ}\right) \in \stackrel{\circ}{\mathcal{R}}\left(P_{1}, P_{2}, N, \mathrm{~K}_{W_{1} W_{2}}\right)
$$

and show that for all sufficiently small $\delta>0$ the rate pair can also be achieved with powers $P_{1}-\delta$ and $P_{2}-\delta$, i.e.,

$$
\left(R_{1}^{\circ}, R_{2}^{\circ}\right) \in \mathcal{R}\left(P_{1}-\delta, P_{2}-\delta, N, \mathrm{~K}_{W_{1} W_{2}}\right) .
$$

We first choose parameters $\eta^{\prime}, \mathbf{a}_{1}^{\prime}, \mathbf{a}_{2}^{\prime}, \mathrm{B}_{1}^{\prime}, \mathrm{B}_{2}^{\prime}, \mathrm{C}^{\prime}$ so that the power constraints (19) are satisfied for powers $P_{1}$ and $P_{2}$ and so that

$$
\left(R_{1}^{\circ}, R_{2}^{\circ}\right) \in \mathcal{R}\left(N, \mathrm{~K}_{W_{1} W_{2}} ; \eta^{\prime}, \mathbf{a}_{1}^{\prime}, \mathbf{a}_{2}^{\prime}, \mathrm{B}_{1}^{\prime}, \mathrm{B}_{2}^{\prime}, \mathrm{C}^{\prime}\right) .
$$

By (64), such a choice always exists. Moreover, for such a choice the matrix $C^{\prime}$ differs from the all-zero matrix and both vectors $\mathbf{a}_{1}^{\prime}$ and $\mathbf{a}_{2}^{\prime}$ differ from the all-zero vector. This can be argued as follows. It is easily shown that if $C^{\prime}=0, \mathbf{a}_{1}^{\prime}=\mathbf{0}$, or $\mathbf{a}_{2}^{\prime}=\mathbf{0}$ then the region $\mathcal{R}\left(N, \mathrm{~K}_{W_{1} W_{2}} ; \eta^{\prime}, \mathbf{a}_{1}^{\prime}, \mathbf{a}_{2}^{\prime}, \mathrm{B}_{1}^{\prime}, \mathrm{B}_{2}^{\prime}, \mathrm{C}^{\prime}\right)$ is degenerate, i.e., either $R_{1}=0$ for all points in the region or $R_{2}=0$ for all points in the region. Consequently, the region $\mathcal{R}\left(N, \mathrm{~K}_{W_{1} W_{2}} ; \eta^{\prime}, \mathbf{a}_{1}^{\prime}, \mathbf{a}_{2}^{\prime}, \mathrm{B}_{1}^{\prime}, \mathrm{B}_{2}^{\prime}, \mathrm{C}^{\prime}\right)$ cannot contain any interior points of $\mathcal{R}\left(P_{1}, P_{2}, N, \mathrm{~K}_{W_{1} W_{2}}\right)$, thus contradicting (66).

We next define for each $\delta>0$ the quantities $\kappa_{1}(\delta)$ and $\kappa_{2}(\delta)$ as in (67a) and (67b), shown at the bottom of the next page, and we define

$$
\kappa(\delta)=\min \left\{\kappa_{1}(\delta), \kappa_{2}(\delta)\right\} .
$$

Since $\mathbf{a}_{1}^{\prime}$ and $\mathbf{a}_{2}^{\prime}$ both differ from $\mathbf{0}$, the denominators in (67a) and (67b) are nonzero and the quantities $\kappa_{1}(\delta), \kappa_{2}(\delta)$, and $\kappa(\delta)$ are well defined. Moreover, $\kappa(\delta)$ tends to 1 as $\delta \downarrow 0$.

The desired inclusion (65) is then established by showing that for all sufficiently small $\delta>0$ the following two statements hold.

i) The parameters $\eta^{\prime}, \kappa(\delta) \mathbf{a}_{1}^{\prime}, \kappa(\delta) \mathbf{a}_{2}^{\prime}, \mathrm{B}_{1}^{\prime}, \mathrm{B}_{2}^{\prime}, \mathrm{C}^{\prime}$ satisfy the power constraints (19) for powers $\left(P_{1}-\delta\right)$ and $\left(P_{2}-\delta\right)$.

ii) The rate pair $\left(R_{1}^{\circ}, R_{2}^{\circ}\right)$ lies in the region $\mathcal{R}\left(N, \mathrm{~K}_{W_{1} W_{2}} ; \eta^{\prime}, \kappa(\delta) \mathbf{a}_{1}^{\prime}, \kappa(\delta) \mathbf{a}_{2}^{\prime}, \mathrm{B}_{1}^{\prime}, \mathrm{B}_{2}^{\prime}, \mathrm{C}^{\prime}\right)$.

Statement i) is easily verified by substituting the parameters $\eta^{\prime}$, $\kappa(\delta) \mathbf{a}_{1}^{\prime}, \kappa(\delta) \mathbf{a}_{2}^{\prime}, \mathbf{B}_{1}^{\prime}, \mathbf{B}_{2}^{\prime}, \mathbf{C}^{\prime}$ into the LHSs of the power constraints (19) and using the fact that the parameters $\eta^{\prime}, \mathbf{a}_{1}^{\prime}, \mathbf{a}_{2}^{\prime}$, $\mathrm{B}_{1}^{\prime}, \mathrm{B}_{2}^{\prime}, \mathrm{C}^{\prime}$ satisfy these power constraints for powers $P_{1}$ and $P_{2}$. Statement ii) follows because for given parameters $\eta, B_{1}$, $\mathrm{B}_{2}$, and $\mathrm{C}$ the RHSs of Constraints (18) - which define the region $\mathcal{R}\left(P_{1}, P_{2}, N, \mathrm{~K}_{W_{1} W_{2}} ;\right)$ when $\mathrm{C} \neq 0$-are continuous in the entries of $\mathbf{a}_{1}$ and $\mathbf{a}_{2}$, and because $\kappa(\delta)$ tends to 1 as $\delta \downarrow 0$.

We finally prove Part 3), i.e., (20). The inclusion of the LHS in the RHS is trivial, because replacing the intersection on the LHS by the specific choice $\mathrm{K}=0$ can only increase the region, 
and because the region $\mathcal{R}\left(P_{1}, P_{2}, N, 0\right)$ is closed. The interesting inclusion is that the LHS contains the RHS. To prove this inclusion, we first notice that

$$
\begin{aligned}
& \operatorname{cl}\left(\bigcup_{\sigma^{2}>0} \bigcap_{\mathrm{K} \succeq 0: \operatorname{tr}(\mathrm{K}) \leq \sigma^{2}} \mathcal{R}\left(P_{1}, P_{2}, N, \mathrm{~K}\right)\right) \\
& \quad \supseteq \operatorname{cl}\left(\bigcup_{\sigma^{2}>0} \bigcap_{\mathrm{K} \succeq 0: \operatorname{tr}(\mathrm{K}) \leq \sigma^{2}} \mathcal{R}\left(P_{1}, P_{2}, N, \operatorname{tr}(\mathrm{K}) \mathrm{I}_{2}\right)\right) \\
& \quad=\operatorname{cl}\left(\bigcup_{\sigma^{2}>0} \mathcal{R}\left(P_{1}, P_{2}, N, \sigma^{2} \mathrm{I}_{2}\right)\right)
\end{aligned}
$$

where the inclusion and the equality both follow by the monotonicity proved in Part 1). Thus, it remains to show that

$$
\operatorname{cl}\left(\bigcup_{\sigma^{2}>0} \mathcal{R}\left(P_{1}, P_{2}, N, \sigma^{2} \mathbf{I}_{2}\right)\right) \supseteq \mathcal{R}\left(P_{1}, P_{2}, N, 0\right) \text {. }
$$

To prove (68), we fix a rate pair $\left(R_{1}^{\circ}, R_{2}^{\circ}\right)$ in the interior of $\mathcal{R}\left(P_{1}, P_{2}, N, 0\right)$, and show that for all sufficiently small $\sigma^{2}>0$ there exists a set of parameters $\eta, \mathbf{a}_{1}, \mathbf{a}_{2}, B_{1}, B_{2}, C$ satisfying the following two statements.

i) The parameters $\eta, \mathbf{a}_{1}, \mathbf{a}_{2}, \mathrm{~B}_{1}, \mathrm{~B}_{2}, \mathrm{C}$ satisfy the power constraints (19) for feedback-noise covariance matrix $\mathrm{K}_{W_{1} W_{2}}=\sigma^{2} \mathrm{I}_{2}$ and powers $P_{1}$ and $P_{2}$.

ii) The rate pair $\left(R_{1}^{\circ}, R_{2}^{\circ}\right)$ lies in the region $\mathcal{R}\left(N, \sigma^{2} \mathrm{I}_{2} ; \eta, \mathbf{a}_{1}, \mathbf{a}_{2}, \mathrm{~B}_{1}, \mathrm{~B}_{2}, \mathrm{C}\right)$.

We first notice that by Part 2), for all sufficiently small $\delta>0$ the pair $\left(R_{1}^{\circ}, R_{2}^{\circ}\right)$ lies in the interior of $\mathcal{R}\left(P_{1}-\delta, P_{2}-\delta, N, 0\right)$, i.e.,

$$
\left(R_{1}^{\circ}, R_{2}^{\circ}\right) \in \stackrel{\circ}{\mathcal{R}}\left(P_{1}-\delta, P_{2}-\delta, N, 0\right) .
$$

This implies that for all sufficiently small $\delta>0$ there exists a set of parameters $\left(\eta(\delta), \mathbf{a}_{1}(\delta), \mathbf{a}_{2}(\delta), \mathrm{B}_{1}(\delta), \mathrm{B}_{2}(\delta), \mathrm{C}(\delta)\right)$ so that

- the power constraints (19) are satisfied for feedback-noise covariance matrix $\mathrm{K}_{W_{1} W_{2}}=0$ and powers $\left(P_{1}-\delta\right)$ and $\left(P_{2}-\delta\right)$

- the rate pair $\left(R_{1}^{\circ}, R_{2}^{\circ}\right)$ satisfies

$$
\begin{aligned}
& \left(R_{1}^{\circ}, R_{2}^{\circ}\right) \\
& \quad \in \stackrel{\circ}{\mathcal{R}}\left(N, 0 ; \eta(\delta), \mathbf{a}_{1}(\delta), \mathbf{a}_{2}(\delta), \mathrm{B}_{1}(\delta), \mathrm{B}_{2}(\delta), \mathrm{C}(\delta)\right) .
\end{aligned}
$$

The proof is then established by fixing a sufficiently small $\delta>$ 0 , and showing that for all sufficiently small $\sigma^{2}>0$ the choice of parameters $\left(\eta(\delta), \mathbf{a}_{1}(\delta), \mathbf{a}_{2}(\delta), \mathrm{B}_{1}(\delta), \mathrm{B}_{2}(\delta), \mathbf{C}(\delta)\right)$ satisfies the above Statements i) and ii).

Statement i) holds because for $\mathrm{K}_{W_{1} W_{2}}=\sigma^{2} \mathrm{I}_{2}$ the LHSs of the power constraints (19) are continuous in $\sigma^{2}>0$, and because the parameters $\left(\eta(\delta), \mathbf{a}_{1}(\delta), \mathbf{a}_{2}(\delta), \mathrm{B}_{1}(\delta), \mathrm{B}_{2}(\delta), \mathrm{C}(\delta)\right)$ satisfy the power constraints for feedback-noise covariance matrix $\mathrm{K}_{W_{1} W_{2}}=0$ and powers $\left(P_{1}-\delta\right)$ and $\left(P_{2}-\delta\right)$. Statement ii) holds because for $\mathrm{K}_{W_{1} W_{2}}=\sigma^{2} \mathrm{I}_{2}$ the RHSs of Constraints (18) - which define the region region $\mathcal{R}\left(N, \mathrm{~K}_{W_{1} W_{2}} ; \eta, \mathbf{a}_{1}, \mathbf{a}_{2}, \mathrm{~B}_{1}, \mathrm{~B}_{2}, \mathrm{C}\right)$ when $\mathrm{C} \neq 0$-are continuous in $\sigma^{2}$, and because of Inclusion (69).

2) Proof of Remark IV.8: Fix $P_{1}, P_{2}, N>0$. Specializing our concatenated scheme to the specific choice of parameters in Remark F.3 in Appendix F obviously cannot outperform our concatenated scheme for general parameters. Thus

$$
\operatorname{cl}\left(\bigcup_{\eta \in \mathbb{N}} \tilde{\mathcal{R}}_{\eta}\left(P_{1}, P_{2}, N, 0\right)\right) \subseteq \mathcal{R}\left(P_{1}, P_{2}, N, 0\right) .
$$

We shall show in the following that

$$
\mathcal{R}_{\mathrm{Oz}}^{\rho^{*}}\left(P_{1}, P_{2}, N\right) \subseteq \mathrm{cl}\left(\bigcup_{\eta \in \mathbb{N}} \tilde{\mathcal{R}}_{\eta}\left(P_{1}, P_{2}, N, 0\right)\right)
$$

which, combined with (70), establishes the remark.

Recall that for fixed $\eta \in \mathbb{N}$ the region $\tilde{\mathcal{R}}_{\eta}\left(P_{1}, P_{2}, N, 0\right)$ is defined as the set of all rate pairs $\left(R_{1}, R_{2}\right)$ satisfying Constraints (72a)-(72c), shown at the bottom of the next page, where

$$
\begin{aligned}
\rho_{1}= & -\rho^{*}\left(P_{1}, P_{2}, N\right) \\
& \text { and for } \ell \in\{2, \ldots, \eta-1\}: \\
\rho_{\ell}= & \frac{\rho_{\ell-1} N-(-1)^{\ell-1} \sqrt{P_{1} P_{2}}\left(1-\rho_{\ell-1}^{2}\right)}{\sqrt{P_{1}\left(1-\rho_{\ell-1}^{2}\right)+N} \sqrt{P_{2}\left(1-\rho_{\ell-1}^{2}\right)+N}}
\end{aligned}
$$

and where $r$ is the unique solution in $[0,1]$ to $(185)$, i.e., to

$$
\sqrt{\frac{r^{2} P_{1} P_{2}}{\left(r P_{1}+N\right)\left(r P_{2}+N\right)}}=\rho^{*}\left(P_{1}, P_{2}, N\right) .
$$

We shall shortly prove that the solution to the recursion (73) is

$$
\rho_{\ell}=(-1)^{\ell} \rho^{*}\left(P_{1}, P_{2}, N\right), \quad \ell \in \mathbb{N} \text {. }
$$

$$
\begin{aligned}
& \kappa_{1}(\delta) \triangleq \sqrt{1-\frac{\delta \eta}{\operatorname{tr}\left(\left(\begin{array}{ll}
\mathrm{I}_{\eta} & 0
\end{array}\right)\left(\mathrm{I}_{2 \eta}-\mathrm{B}_{\mathrm{b}}\right)^{-1} \mathrm{~A}_{\mathrm{d}} \mathrm{A}_{\mathrm{d}}^{\mathrm{T}}\left(\mathrm{I}_{2 \eta}-\mathrm{B}_{\mathrm{b}}\right)^{-\mathrm{T}}\left(\begin{array}{c}
\mathrm{I}_{\eta} \\
0
\end{array}\right)\right)}} \\
& \kappa_{2}(\delta) \triangleq \sqrt{1-\frac{\delta \eta}{\operatorname{tr}\left(\left(\begin{array}{ll}
0 & \mathrm{I}_{\eta}
\end{array}\right)\left(\mathrm{I}_{2 \eta}-\mathrm{B}_{\mathrm{b}}\right)^{-1} \mathrm{~A}_{\mathrm{d}} \mathrm{A}_{\mathrm{d}}^{\mathrm{T}}\left(\mathrm{I}_{2 \eta}-\mathrm{B}_{\mathrm{b}}\right)^{-\mathrm{T}}\left(\begin{array}{c}
0 \\
\mathrm{I}_{\eta}
\end{array}\right)\right)}}
\end{aligned}
$$


This implies that for all $\ell \in \mathbb{N}$ larger than 1:

$$
\begin{aligned}
\rho_{\ell-1}^{2} & =\rho^{* 2}, \\
(-1)^{\ell-1} \rho_{\ell-1} & =\rho^{*}
\end{aligned}
$$

and hence for fixed $\eta \in \mathbb{N}$ the region $\tilde{\mathcal{R}}_{\eta}\left(P_{1}, P_{2}, N, 0\right)$ contains all rate pairs $\left(R_{1}, R_{2}\right)$ satisfying

$$
\begin{aligned}
R_{1} \leq & \frac{1}{2 \eta} \log \left(1+\frac{r P_{1}}{N}\right) \\
& +\frac{\eta-1}{2 \eta} \log \left(1+\frac{P_{1}\left(1-\rho^{* 2}\right)}{N}\right) \\
R_{2} \leq & \frac{1}{2 \eta} \log \left(1+\frac{r P_{2}}{N}\right) \\
& +\frac{\eta-1}{2 \eta} \log \left(1+\frac{P_{2}\left(1-\rho^{* 2}\right)}{N}\right) \\
R_{1}+R_{2} \leq & \frac{1}{2 \eta} \log \left(1+\frac{r P_{1}+r P_{2}}{N}\right) \\
& +\frac{\eta-1}{2 \eta} \log \left(1+\frac{P_{1}+P_{2}+2 \sqrt{P_{1} P_{2}} \rho^{*}}{N}\right)
\end{aligned}
$$

Notice that when $\eta$ tends to infinity, the RHSs of (75a)-(75c) tend to the RHSs of the three Constraints (7a)-(7c) evaluated for $\rho=\rho^{*}$. Since Constraints (7a)-(7c) evaluated for $\rho=\rho^{*}$ determine the region $\mathcal{R}_{\mathrm{Oz}}^{\rho^{*}}\left(P_{1}, P_{2}, N\right)$, Inclusion (71) follows immediately by (75) and by letting $\eta$ tend to infinity.

In the remaining, we prove (74) in two steps. In the first step we show that $\rho^{*}\left(P_{1}, P_{2}, N\right)$ is a fix point of the function $h(\cdot)$ defined as

$$
\begin{aligned}
h & :[0,1] \rightarrow \mathbb{R}, \\
h(\rho) & =\frac{\sqrt{P_{1} P_{2}}\left(1-\rho^{2}\right)-\rho N}{\sqrt{P_{1}\left(1-\rho^{2}\right)+N} \sqrt{P_{2}\left(1-\rho^{2}\right)+N}} .
\end{aligned}
$$

Notice that $h(\cdot)$ has at least one fix point in $[0,1]$ because $h(0)>0$ whereas $h(1)<0$, and because $h(\cdot)$ is continuous. Further notice that every fix point of $h(\cdot)$ must also be a solution to

$$
1-h(\rho)^{2}=1-\rho^{2}
$$

i.e., a solution to

$$
\frac{N\left(N+P_{1}+P_{2}+2 \sqrt{P_{1} P_{2}} \rho\right)}{\left(N+P_{1}\left(1-\rho^{2}\right)\right)\left(N+P_{2}\left(1-\rho^{2}\right)\right)}\left(1-\rho^{2}\right)=\left(1-\rho^{2}\right) .
$$

The solutions in $[0,1]$ to (76) are given by $\rho=1$ and by the solutions to

$$
\begin{aligned}
N\left(N+P_{1}\right. & \left.+P_{2}+2 \sqrt{P_{1} P_{2}} \rho\right) \\
& =\left(N+P_{1}\left(1-\rho^{2}\right)\right)\left(N+P_{2}\left(1-\rho^{2}\right)\right) .
\end{aligned}
$$

Since $\rho=1$ is not a fix point of $h(\cdot)$ and since $\rho^{*}\left(P_{1}, P_{2}, N\right)$ is the unique solution in $[0,1]$ to (77) (see Definition III.1), $\rho^{*}\left(P_{1}, P_{2}, N\right)$ must be a fix point of $h(\cdot)$. This concludes the first step.

In the second step we use the derived fix-point property of $h(\cdot)$ to prove (74). The proof is lead by induction. For $\ell=1$ Condition (74) holds by definition. Assuming that (74) holds for some fixed $\ell \geq 1$, we have

$$
\begin{aligned}
& \rho_{\ell+1} \\
& =\frac{-(-1)^{\ell} \sqrt{P_{1} P_{2}}\left(1-\left|\rho_{\ell}\right|^{2}\right)+\rho_{\ell} N}{\sqrt{P_{1}\left(1-\left|\rho_{\ell}\right|^{2}\right)+N} \sqrt{P_{2}\left(1-\left|\rho_{\ell}\right|^{2}+N\right.}} \\
& =(-1)^{\ell+1} \frac{\sqrt{P_{1} P_{2}}\left(1-\left|\rho_{\ell}\right|^{2}\right)-\left|\rho_{\ell}\right| N}{\sqrt{P_{1}\left(1-\left|\rho_{\ell}\right|^{2}\right)+N} \sqrt{P_{2}\left(1-\left|\rho_{\ell}\right|^{2}\right)+N}} \\
& =(-1)^{\ell+1} h\left(\left|\rho_{\ell}\right|\right) \\
& =(-1)^{\ell+1} \rho^{*}\left(P_{1}, P_{2}, N\right),
\end{aligned}
$$

where (78) follows by the definition of the sequence $\left\{\rho_{\ell}\right\}$ for $\ell>1$; (79) follows because by the induction assumption $\operatorname{sign}\left(\rho_{\ell}\right)=(-1)^{\ell} ;(80)$ follows by the definition of the function $h(\cdot)$; and finally $(81)$ follows because by the induction assumption $\left|\rho_{\ell}\right|=\rho^{*}$ and because $\rho^{*}$, as shown in the first step, is a fix point of $h(\cdot)$. Thus, (74) holds also for $(\ell+1)$, which concludes the induction step and the proof of the remark.

3) Proof of Remark IV.15: We only prove (26a); (26b) can be proved analogously.

Fix $\rho \in\left[0, \rho^{*}\right]$, and define $\alpha(\rho)$ as the unique solution in $[0,1[$ to

$$
\frac{P_{1}+P_{2}+2 \sqrt{P_{1} P_{2}} \rho+N}{P_{1}\left(1-\rho^{2}\right)+N}=1+\frac{P_{2}\left(1-\frac{\rho^{2}}{1-\alpha}\right)}{\alpha P_{1}+N} .
$$

$$
\begin{aligned}
R_{1} & \leq \frac{1}{2 \eta} \log \left(1+\frac{r P_{1}}{N}\right)+\sum_{\ell=2}^{\eta} \frac{1}{2 \eta} \log \left(1+\frac{P_{1}\left(1-\rho_{\ell-1}^{2}\right)}{N}\right) \\
R_{2} & \leq \frac{1}{2 \eta} \log \left(1+\frac{r P_{2}}{N}\right)+\sum_{\ell=2}^{\eta} \frac{1}{2 \eta} \log \left(1+\frac{P_{2}\left(1-\rho_{\ell-1}^{2}\right)}{N}\right) \\
R_{1}+R_{2} & \leq \frac{1}{2 \eta} \log \left(1+\frac{r P_{1}+r P_{2}}{N}\right)+\sum_{\ell=2}^{\eta} \frac{1}{2 \eta} \log \left(1+\frac{P_{1}+P_{2}+2 \sqrt{P_{1} P_{2}}(-1)^{\ell-1} \rho_{\ell-1}}{N}\right)
\end{aligned}
$$


That (82) has exactly one solution in $[0,1)$ follows by the Intermediate Value Theorem and the following observations. The RHS of (82) is continuous and strictly decreasing in $\alpha$; for $\alpha=0$ the RHS of (82) is larger or equal to the LHS because $0 \leq \rho \leq$ $\rho^{*}$ and by Remark III.3; and for $\alpha$ tending to 1 the RHS tends to $-\infty$ and thus is smaller than the LHS.

Further, define

$$
\begin{aligned}
P_{1}^{\prime} & \triangleq \alpha(\rho) P_{1}, \\
P_{1}^{\prime \prime} & \triangleq(1-\alpha(\rho)) P_{1}, \\
N^{\prime} & \triangleq P_{1}^{\prime}+N, \\
\rho^{\prime} & \triangleq \frac{\rho}{\sqrt{1-\alpha(\rho)}}
\end{aligned}
$$

and notice that by these definitions:

$$
\begin{aligned}
N^{\prime}\left(N^{\prime}+P_{1}^{\prime \prime}+P_{2}+2 \sqrt{P_{1}^{\prime \prime} P_{2}} \rho^{\prime}\right) \\
=\left(N^{\prime}+P_{1}^{\prime \prime}\left(1-\rho^{\prime 2}\right)\right)\left(N^{\prime}+P_{2}\left(1-\rho^{\prime 2}\right)\right)
\end{aligned}
$$

and hence

$$
\rho^{\prime}=\rho^{*}\left(P_{1}^{\prime \prime}, P_{2}, N^{\prime}\right) .
$$

Also, define $\left(R_{1, \mathrm{Oz}}^{\rho}, R_{2, \mathrm{Oz}}^{\rho}\right)$ as the dominant corner point of the rectangle $\mathcal{R}_{1, \mathrm{Oz}}^{\rho}\left(P_{1}, P_{2}, N\right)$. The following two remarks on $\left(R_{1, \mathrm{Oz}}^{\rho}, R_{2, \mathrm{Oz}}^{\rho}\right)$ are from [13], and based on (83).

Remark IV.18: The rate point $\left(R_{1, \mathrm{Oz}}^{\rho}, R_{2, \mathrm{Oz}}^{\rho}\right)$ can be expressed as

$$
\begin{aligned}
& R_{1, \mathrm{Oz}}^{\rho}=R_{1,1, \mathrm{Oz}}^{\rho}+R_{1,2, \mathrm{Oz}}^{\rho}, \\
& R_{2, \mathrm{Oz}}^{\rho}=\frac{1}{2} \log \left(1+\frac{P_{2}\left(1-\rho^{\prime 2}\right)}{N^{\prime}}\right),
\end{aligned}
$$

where

$$
\begin{aligned}
& R_{1,1, \mathrm{Oz}}^{\rho} \triangleq \frac{1}{2} \log \left(1+\frac{P_{1}^{\prime}}{N}\right), \\
& R_{1,2, \mathrm{Oz}}^{\rho} \triangleq \frac{1}{2} \log \left(1+\frac{P_{1}^{\prime \prime}\left(1-\rho^{\prime 2}\right)}{N^{\prime}}\right) .
\end{aligned}
$$

Remark IV.19: The rate point $\left(R_{1,2, \mathrm{Oz}}^{\rho}, R_{2, \mathrm{Oz}}^{\rho}\right)$ corresponds to the dominant corner point of the rectangle $\mathcal{R}_{\mathrm{Oz}}^{\rho^{\prime}}\left(P_{1}^{\prime \prime}, P_{2}, N^{\prime}\right)$, where $\rho^{\prime}=\rho^{*}\left(P_{1}^{\prime \prime}, P_{2}, N^{\prime}\right)$. We are now ready to prove Inclusion (26a). For $\mathrm{K}_{W_{1} W_{2}}=0$ the RHS of (24) equals $\frac{1}{2} \log \left(1+\frac{P_{1}^{\prime}}{N}\right)$, irrespective of the parameters $\mathbf{a}_{1}, \mathbf{a}_{2}, \mathrm{~B}_{1}, \mathrm{~B}_{2}, \mathrm{C}$. Therefore, the region $\mathcal{R}_{\mathrm{RS}, 1}\left(P_{1}^{\prime}, P_{1}^{\prime \prime}, P_{2}, N, 0\right)$ is given by the set of all rate pairs $\left(R_{1}, R_{2}\right)$ which for some nonnegative $R_{1, \mathrm{CS}}, R_{1, \mathrm{NF}}$ summing to $R_{1}$ satisfy

$$
\begin{aligned}
\left(R_{1, \mathrm{CS}}, R_{2}\right) & \in \mathcal{R}\left(P_{1}^{\prime \prime}, P_{2}, N^{\prime}, 0\right), \\
R_{1, \mathrm{NF}} & \leq \frac{1}{2} \log \left(1+\frac{P_{1}^{\prime}}{N}\right) .
\end{aligned}
$$

Since by Remark IV.19 and Remark IV.8:

$$
\left(R_{1,2, \mathrm{Oz}}^{\rho}, R_{2, \mathrm{Oz}}^{\rho}\right) \in \mathcal{R}\left(P_{1}^{\prime \prime}, P_{2}, N^{\prime}, 0\right)
$$

and by Remark IV.18:

$$
R_{1,1, \mathrm{Oz}}^{\rho} \leq \frac{1}{2} \log \left(1+\frac{P_{1}^{\prime}}{N}\right)
$$

the triple $\left(R_{1,1, \mathrm{Oz}}^{\rho}, R_{1,2, \mathrm{Oz}}^{\rho}, R_{2, \mathrm{Oz}}^{\rho}\right)$ satisfies (84), and hence

$$
\left(R_{1, \mathrm{Oz}}^{\rho}, R_{2, \mathrm{Oz}}^{\rho}\right) \in \mathcal{R}_{\mathrm{RS}, 1}\left(P_{1}^{\prime}, P_{1}^{\prime \prime}, P_{2}, N, 0\right) .
$$

Inclusion (26a) finally follows because $\left(R_{1, \mathrm{Oz}}^{\rho}, R_{2, \mathrm{Oz}}^{\rho}\right)$ is the dominant corner point of the rectangle $\mathcal{R}_{1, \mathrm{Oz}}^{\rho}\left(P_{1}, P_{2}, N\right)$, and therefore (85) implies that the entire region $\mathcal{R}_{1, \mathrm{Oz}}^{\rho}\left(P_{1}, P_{2}, N\right)$ is contained in $\mathcal{R}_{\mathrm{RS}, 1}\left(P_{1}^{\prime}, P_{1}^{\prime \prime}, P_{2}, N, 0\right)$.

4) Proof of Proposition IV.16: We only prove (27a); (27b) can be proved analogously.

To this end, fix a $\rho \in\left[0, \rho^{*}\left(P_{1}, P_{2}, N\right)\right]$ and choose a power $P_{1}^{\prime} \in\left[0, P_{1}\right]$ such that

$$
\mathcal{R}_{\mathrm{RS}, 1}\left(P_{1}^{\prime},\left(P_{1}-P_{1}^{\prime}\right), P_{2}, N, 0\right) \supseteq \mathcal{R}_{1, \mathrm{Oz}}^{\rho}\left(P_{1}, P_{2}, N\right) .
$$

Notice that by Remark IV.15 such a power $P_{1}^{\prime}$ always exists. Inclusion (27a) follows then because by Proposition IV.14, Part 2:

$$
\begin{aligned}
\operatorname{cl}\left(\bigcup_{\sigma^{2}>0} \bigcap_{\substack{\mathrm{K} \succeq 0: \\
\operatorname{tr}(\mathrm{K}) \leq \sigma^{2}}} \mathcal{R}_{\mathrm{RS}, 1}\left(P_{1}^{\prime},\left(P_{1}-P_{1}^{\prime}\right), P_{2}, N, \mathrm{~K}\right)\right) \\
=\mathcal{R}_{\mathrm{RS}, 1}\left(P_{1}^{\prime},\left(P_{1}-P_{1}^{\prime}\right), P_{2}, N, 0\right) .
\end{aligned}
$$

5) Proof of Theorem IV.17: Fix $P_{1}, P_{2}, N>0$. The proof of the $\subseteq$-direction follows trivially because replacing the intersection on the LHS by the specific choice $\mathrm{K}=0$ can only increase the region, because $\mathrm{C}_{\text {NoisyFB }}\left(P_{1}, P_{2}, N, 0\right)=\mathrm{C}_{\text {PerfectFB }}\left(P_{1}, P_{2}, N\right)$, and because by definition the region $\mathrm{C}_{\text {PerfectFB }}\left(P_{1}, P_{2}, N\right)$ is closed. The $\supseteq$-direction, i.e.,

$$
\begin{array}{r}
\operatorname{cl}\left(\bigcup_{\sigma^{2}>0} \bigcap_{\substack{\mathrm{K} \succeq 0: \\
\operatorname{tr}(\mathrm{K}) \leq \sigma^{2}}} \mathrm{C}_{\text {NoisyFB }}\left(P_{1}, P_{2}, N, \mathrm{~K}\right)\right) \\
\supseteq \mathrm{C}_{\text {PerfectFB }}\left(P_{1}, P_{2}, N\right),
\end{array}
$$

follows from (87)-(90), shown at the bottom of the next page, where (87) follows from Proposition IV.13, (88) follows by basic rules on sets, (89) follows from Proposition IV.16, and (90) follows by Remark III.7.

\section{PARTIAL FEEDBACK}

We now focus on the setup with noisy or perfect partial feedback. For this setup we again present new achievable regions, and based on these new regions we derive new qualitative properties of the capacity region (Section V-A). We also present the coding schemes corresponding to these new achievable regions (Sections V-B-D). They are obtained from the noisy-feedback schemes in Sections IV-B-D by restricting the set of parameters 
and in the case of the extended schemes by additionally specializing Carleial's scheme to noisy partial feedback.

\section{A. Results}

We first present results for noisy partial feedback (Section V-A1) and then results that hold only for perfect partial feedback (Section V-A2).

1) Results for Noisy Partial Feedback: Evaluating the rates achieved by our concatenated scheme with general parameters in Section V-C1 ahead leads to the achievability result in Theorem V.3. Before stating the result we define:

Definition V.1: Let $\eta$ be a positive integer, let $\mathbf{a}_{1}, \mathbf{a}_{2}$ be $\eta$-dimensional vectors, let $\mathrm{B}_{2}$ be a strictly lower-triangular $\eta \times \eta$ matrix, and let $C_{P}$ be a $2 \times \eta$ matrix. Then, depending on the matrix $\mathrm{C}_{\mathrm{P}}$ the rate region $\mathcal{R}_{\mathrm{P}}\left(N, \sigma_{2}^{2} ; \eta, \mathbf{a}_{1}, \mathbf{a}_{2}, \mathrm{~B}_{2}, \mathrm{C}_{\mathrm{P}}\right)$ is defined as follows.

- If the product $\mathrm{C}_{\mathrm{P}} \mathrm{C}_{\mathrm{P}}^{\mathrm{T}}$ is nonsingular, ${ }^{11}$ then $\mathcal{R}_{\mathrm{P}}\left(N, \sigma_{2}^{2} ; \eta, \mathbf{a}_{1}, \mathbf{a}_{2}, \mathrm{~B}_{2}, \mathrm{C}_{\mathrm{P}}\right)$ is defined as the set of all rate pairs $\left(R_{1}, R_{2}\right)$ satisfying

$$
\begin{aligned}
R_{1} & \leq \frac{1}{2 \eta} \log \frac{\left|\mathrm{C}_{\mathrm{P}}\left(\mathbf{a}_{1} \mathbf{a}_{1}^{\top}+N \mathrm{I}_{\eta}+\sigma_{2}^{2} \mathrm{~B}_{2} \mathrm{~B}_{2}^{\top}\right) \mathrm{C}_{\mathrm{P}}^{\mathrm{T}}\right|}{\left|\mathrm{C}_{\mathrm{P}}\left(N \mathrm{I}_{\eta}+\sigma_{2}^{2} \mathrm{~B}_{2} \mathrm{~B}_{2}^{\top}\right) \mathrm{C}_{\mathrm{P}}^{\mathrm{T}}\right|}, \\
R_{2} & \leq \frac{1}{2 \eta} \log \frac{\left|\mathrm{C}_{\mathrm{P}}\left(\mathbf{a}_{2} \mathbf{a}_{2}^{\top}+N \mathrm{I}_{\eta}+\sigma_{2}^{2} \mathrm{~B}_{2} \mathrm{~B}_{2}^{\top}\right) \mathrm{C}_{\mathrm{P}}^{\mathrm{T}}\right|}{\left|\mathrm{C}_{\mathrm{P}}\left(N \mathrm{I}_{\eta}+\sigma_{2}^{2} \mathrm{~B}_{2} \mathrm{~B}_{2}^{\top}\right) \mathrm{C}_{\mathrm{P}}^{\mathrm{T}}\right|} \\
R_{1} & +R_{2} \\
& \leq \frac{1}{2 \eta} \log \frac{\left|\mathrm{C}_{\mathrm{P}}\left(\mathrm{A}_{\mathrm{r}} \mathrm{A}_{\mathrm{r}}^{\top}+N \mathrm{I}_{\eta}+\sigma_{2}^{2} \mathrm{~B}_{2} \mathrm{~B}_{2}^{\top}\right) \mathrm{C}_{\mathrm{P}}^{\top}\right|}{\left|\mathrm{C}_{\mathrm{P}}\left(N \mathrm{I}_{\eta}+\sigma_{2}^{2} \mathrm{~B}_{2} \mathrm{~B}_{2}^{\top}\right) \mathrm{C}_{\mathrm{P}}^{\top}\right|}
\end{aligned}
$$

where $A_{r}$ is defined in (11).

\footnotetext{
${ }^{11}$ Whenever $\eta \in \mathbb{N}$ is larger than 1 , there is no loss in optimality in restricting attention to matrices $C_{P}$ so that $C_{P} C_{P}^{T}$ is nonsingular.
}

- If the product $C_{P} C_{P}^{T}$ is singular but $C_{P} \neq 0$, then $\mathcal{R}_{\mathrm{P}}\left(N, \sigma_{2}^{2} ; \eta, \mathbf{a}_{1}, \mathbf{a}_{2}, \mathrm{~B}_{2}, \mathrm{C}_{\mathrm{P}}\right)$ is defined as the set of all rate pairs $\left(R_{1}, R_{2}\right)$ satisfying (91) when the $2 \times \eta$ matrix $\mathrm{C}_{\mathrm{P}}$ is replaced by the $\eta$-dimensional row-vector obtained by choosing one of its nonzero rows.

- If $\mathrm{C}_{\mathrm{P}}=0$, then $\mathcal{R}_{\mathrm{P}}\left(N, \sigma_{2}^{2} ; \eta, \mathbf{a}_{1}, \mathbf{a}_{2}, \mathrm{~B}_{2}, \mathrm{C}_{\mathrm{P}}\right)$ is defined as the set containing only the origin.

An alternative formulation of the region $\mathcal{R}_{\mathrm{P}}\left(N, \sigma_{2}^{2} ; \eta, \mathbf{a}_{1}, \mathbf{a}_{2}, \mathrm{~B}_{2}, \mathrm{C}_{\mathrm{P}}\right)$ is presented in $\mathrm{Sec}$ tion D-B in Appendix D.

Definition V.2: Define

$$
\begin{aligned}
\mathcal{R}_{\mathrm{P}} & \left(P_{1}, P_{2}, N, \sigma_{2}^{2}\right) \\
& \triangleq \operatorname{cl}\left(\bigcup_{\eta, \mathbf{a}_{1}, \mathbf{a}_{2}, \mathrm{~B}_{2}, \mathrm{C}_{\mathrm{P}}} \mathcal{R}_{\mathrm{P}}\left(N, \sigma_{2}^{2} ; \eta, \mathbf{a}_{1}, \mathbf{a}_{2}, \mathrm{~B}_{2}, \mathrm{C}_{\mathrm{P}}\right)\right)
\end{aligned}
$$

where the union is over all tuples $\left(\eta, \mathbf{a}_{1}, \mathbf{a}_{2}, \mathrm{~B}_{2}, \mathrm{C}_{\mathrm{P}}\right)$ satisfying the trace constraints

$$
\mathbf{a}_{1}^{\top} \mathbf{a}_{1} \leq \eta P_{1}
$$

and

$$
\begin{aligned}
& \operatorname{tr}\left(( \mathrm { I } _ { \eta } - \mathrm { B } _ { 2 } ) ^ { - 1 } \left(\mathbf{a}_{2} \mathbf{a}_{2}^{\top}+\mathrm{B}_{2} \mathbf{a}_{1} \mathbf{a}_{1}^{\top} \mathrm{B}_{2}^{\top}\right.\right. \\
& \left.\left.\quad+\left(N+\sigma_{2}^{2}\right) \mathrm{B}_{2} \mathrm{~B}_{2}^{\top}\right)\left(\mathrm{I}_{\eta}-\mathrm{B}_{2}\right)^{-\top}\right) \leq \eta P_{2} .
\end{aligned}
$$

Theorem V.3 (Noisy Partial Feedback): The capacity region $\mathrm{C}_{\text {NoisyPartialFB }}\left(P_{1}, P_{2}, N, \sigma_{2}^{2}\right)$ of the two-user AWGN MAC with noisy partial feedback to Transmitter 2 contains the rate region $\mathcal{R}_{\mathrm{P}}\left(P_{1}, P_{2}, N, \sigma_{2}^{2}\right)$, i.e.,

$$
\mathrm{C}_{\text {NoisyPartialFB }}\left(P_{1}, P_{2}, N, \sigma_{2}^{2}\right) \supseteq \mathcal{R}_{\mathrm{P}}\left(P_{1}, P_{2}, N, \sigma_{2}^{2}\right) \text {. }
$$

$$
\begin{aligned}
& \operatorname{cl}\left(\bigcup_{\sigma^{2}>0 \mathrm{~K} \succeq 0: \operatorname{tr}(\mathrm{K}) \leq \sigma^{2}} \mathrm{C}_{\text {NoisyFB }}\left(P_{1}, P_{2}, N, \mathrm{~K}\right)\right) \\
& \supseteq \operatorname{cl}\left(\bigcup _ { \sigma ^ { 2 } > 0 \mathrm { K } \succeq 0 : \operatorname { t r } ( \mathrm { K } ) \leq \sigma ^ { 2 } } \left(\left(\bigcup_{P_{1}^{\prime} \in\left[0, P_{1}\right]} \mathcal{R}_{\mathrm{RS}, 1}\left(P_{1}^{\prime},\left(P_{1}-P_{1}^{\prime}\right), P_{2}, N, \mathrm{~K}\right)\right)\right.\right. \\
& \left.\left.\cup\left(\bigcup_{P_{2}^{\prime} \in\left[0, P_{2}\right]} \mathcal{R}_{\mathrm{RS}, 2}\left(P_{1}, P_{2}^{\prime},\left(P_{2}-P_{2}^{\prime}\right), N, \mathrm{~K}\right)\right)\right)\right) \\
& \supseteq \operatorname{cl}\left(\bigcup_{P_{1}^{\prime} \in\left[0, P_{1}\right]} \operatorname{cl}\left(\bigcup_{\sigma^{2}>0 \mathrm{~K} \succeq 0: \operatorname{tr}(\mathrm{K}) \leq \sigma^{2}} \mathcal{R}_{\mathrm{RS}, 1}\left(P_{1}^{\prime},\left(P_{1}-P_{1}^{\prime}\right), P_{2}, N, \mathrm{~K}\right)\right)\right) \\
& \cup \operatorname{cl}\left(\bigcup_{P_{2}^{\prime} \in\left[0, P_{2}\right]} \operatorname{cl}\left(\bigcup_{\sigma^{2}>0 \mathrm{~K} \succeq 0: \operatorname{tr}(\mathrm{K}) \leq \sigma^{2}} \mathcal{R}_{\mathrm{RS}, 2}\left(P_{1}, P_{2}^{\prime},\left(P_{2}-P_{2}^{\prime}\right), N, \mathrm{~K}\right)\right)\right) \\
& \supseteq \operatorname{cl}\left(\bigcup_{\rho \in\left[0, \rho^{*}\left(P_{1}, P_{2}, N\right)\right]} \mathcal{R}_{1, \mathrm{Oz}}^{\rho}\left(P_{1}, P_{2}, N\right)\right) \cup \mathrm{cl}\left(\bigcup_{\rho \in\left[0, \rho^{*}\left(P_{1}, P_{2}, N\right)\right]} \mathcal{R}_{2, \mathrm{Oz}}^{\rho}\left(P_{1}, P_{2}, N\right)\right) \\
& =\mathrm{C}_{\text {PerfectFB }}\left(P_{1}, P_{2}, N\right)
\end{aligned}
$$


Proof: Follows from Theorem IV.3 by choosing $\mathrm{B}_{1}$ as the all-zero matrix.

Remark V.4: Evaluating the achievable region $\mathcal{R}_{\mathrm{P}}\left(P_{1}, P_{2}, N, \sigma_{2}^{2}\right)$ seems to be difficult even numerically. More easily computable (but possibly smaller) achievable regions are obtained by taking the union on the RHS of (92) only over a subset of the parameters $\eta, \mathbf{a}_{1}, \mathbf{a}_{2}, \mathrm{~B}_{2}, \mathrm{C}_{\mathrm{P}}$ satisfying (93). In Remark E.1 in Appendix E we present such a subset of parameters. In Section V-C2 we present general guidelines on how to choose the parameters $\eta, \mathbf{a}_{1}, \mathbf{a}_{2}, B_{2}, C_{P}$.

Specializing Theorem V.3 to equal powers channels, i.e., $P_{1}=P_{2}=P$, and to $\eta=2$ and the choice of the parameters presented in Section E-A (Appendix E) yields the following Corollary V.5.

Corollary V.5 (Equal Powers and Noisy Partial Feedback): The capacity region $\mathrm{C}_{\text {NoisyPartialFB }}\left(P, P, N, \sigma_{2}^{2}\right)$ of the twouser AWGN MAC with noisy partial feedback to Transmitter 2 and equal powers $P_{1}=P_{2}=P$ contains all rate pairs $\left(R_{1}, R_{2}\right)$ satisfying (94a)-(94c), shown at the bottom of the page.

From Corollary V.5, it follows immediately that for equalpowers channels noisy partial feedback increases the capacity, no matter how large the noise variance $\sigma^{2} \geq 0$ is. The following stronger result holds.

Theorem V.6 (Noisy Partial Feedback is Always Beneficial): For all $N, P_{1}, P_{2}>0$ and $\sigma_{2}^{2} \geq 0$

$$
\mathrm{C}_{\mathrm{NoFB}}\left(P_{1}, P_{2}, N\right) \subset \mathrm{C}_{\text {NoisyPartialFB }}\left(P_{1}, P_{2}, N, \sigma_{2}^{2}\right)
$$

where the inclusion is strict.

Proof: See Section V-E1.

2) Results for Perfect Partial Feedback: Specializing Theorem V.3 to perfect partial feedback, i.e., to $\sigma_{2}^{2}=0$ yields the following.

Corollary V.7 (Perfect Partial Feedback): The capacity region $\mathrm{C}_{\text {PerfectPartialFB }}\left(P_{1}, P_{2}, N\right)$ of the two-user AWGN MAC with perfect partial feedback to Transmitter 2 contains the rate region $\mathcal{R}_{\mathrm{P}}\left(P_{1}, P_{2}, N, 0\right)$, i.e.,

$$
\mathrm{C}_{\text {PerfectPartialFB }}\left(P_{1}, P_{2}, N\right) \supseteq \mathcal{R}_{\mathrm{P}}\left(P_{1}, P_{2}, N, 0\right) .
$$

Specializing Corollary V.7 to $\eta=2$ and the choice of parameters in Section E-A in Appendix E yields:

Corollary V.8: The capacity region $\mathrm{C}_{\text {PerfectPartialFB }}\left(P_{1}, P_{2}, N\right)$ of the two-user AWGN MAC with perfect partial feedback to Transmitter 2 contains all rate pairs $\left(R_{1}, R_{2}\right)$ satisfying

$$
\begin{aligned}
R_{1} \leq & \frac{1}{4} \log \left(1+\frac{2 P_{1}}{N}\right), \\
R_{2} \leq & \frac{1}{4} \log \left(1+\frac{P_{2}\left(2+\frac{P_{2}}{P_{1}+N}\right)}{N}\right), \\
R_{1}+R_{2} & \\
\leq & \frac{1}{4} \log \left(1+\frac{P_{1}+P_{2}}{N}\right) \\
& +\frac{1}{4} \log \left(1+\frac{P_{1} \frac{P_{2}+N}{P_{1}+P_{2}+N}+P_{2}}{N}\right. \\
& \left.+\frac{2 \sqrt{P_{1} P_{2} \frac{P_{1}}{P_{1}+N} \frac{P_{2}}{P_{1}+P_{2}+N}}}{N}\right) .
\end{aligned}
$$

With this Corollary V.8 at hand we can answer the question by van der Meulen in [18] whether the Cover-Leung region equals the capacity region of the MAC with perfect partial feedback.

Theorem V.9: Consider a two-user AWGN MAC with perfect partial feedback. For some powers $P_{1}, P_{2}$ and noise variance $N$ the inclusion

$$
\mathcal{R}_{\mathrm{CL}}\left(P_{1}, P_{2}, N\right) \subset \mathrm{C}_{\text {PerfectPartialFB }}\left(P_{1}, P_{2}, N\right)
$$

is strict.

Proof: The inclusion is proved in Section V-E2 by showing that for powers $P_{1}=1, P_{2}=5$ and noise variance $N=5$ the region in Corollary V.8 contains rate points that lie strictly outside the Cover-Leung region.

The last two results are achieved by modifying the rate-splitting schemes for noisy feedback in Sections IV-D2 and IV-D3 so as to apply also for perfect partial feedback. For details see Section V-D.

$$
\begin{aligned}
R_{1} \leq \frac{1}{4} \log \left(1+\frac{2 P}{N}\right) & +\frac{1}{4} \log \left(1-\frac{P}{2 P+N} \frac{P \frac{P}{N} \sigma_{2}^{2}}{\left(2 P+N+\sigma_{2}^{2}+\frac{P}{N} \sigma_{2}^{2}\right)\left(P+N+\sigma_{2}^{2}+\frac{P}{N} \sigma_{2}^{2}\right)}\right) \\
R_{2} \leq \frac{1}{4} \log \left(1+\frac{2 P}{N}\right) & +\frac{1}{4} \log \left(1+\frac{P}{2 P+N} \cdot \frac{P}{P+N+\sigma_{2}^{2}+\frac{P}{N} \sigma_{2}^{2}}\right) \\
R_{1}+R_{2} \leq \frac{1}{2} \log \left(1+\frac{2 P}{N}\right) & +\frac{1}{4} \log \left(1+\frac{2 P^{2}}{(2 P+N)^{2}}\left(\sqrt{1+\frac{P\left(P+N+\sigma_{2}^{2}\right)}{\left(P+N+\sigma_{2}^{2}+\frac{P}{N} \sigma_{2}^{2}\right)^{2}}}-1\right)\right. \\
& \left.+\left(\frac{P}{2 P+N}\right)^{2} \frac{(P+N)\left(2 P+N+\sigma_{2}^{2}\right)}{\left(2 P+N+\sigma_{2}^{2}+\frac{P}{N} \sigma_{2}^{2}\right)\left(P+N+\sigma_{2}^{2}+\frac{P}{N} \sigma_{2}^{2}\right)}\right)
\end{aligned}
$$


Proposition V.10 (Rate-Splitting for Perfect Partial Feedback I): The capacity region $\mathrm{C}_{\text {PerfectPartialFB }}\left(P_{1}, P_{2}, N\right)$ of the two-user AWGN MAC with perfect partial feedback to Transmitter 2 contains all rate pairs $\left(R_{1}, R_{2}\right)$ which for some nonnegative $R_{1, \mathrm{CL}}, R_{1, \mathrm{CS}}$ summing to $R_{1}$, for some nonnegative $R_{2, \mathrm{CL}}, R_{2, \mathrm{CS}}$ summing to $R_{2}$, and for some choice of $\rho_{1}, \rho_{2} \in[0,1]$ and $P_{1}^{\prime} \in\left[0, P_{1}\right], P_{2}^{\prime} \in\left[0, P_{2}\right]$ satisfy

$$
\begin{aligned}
& \left(R_{1, \mathrm{CL}}, R_{2, \mathrm{CL}}\right) \in \mathcal{R}_{\mathrm{CL}}^{\left(\rho_{1}, \rho_{2}\right)}\left(P_{1}^{\prime}, P_{2}^{\prime}, N\right), \\
& \left(R_{1, \mathrm{CS}}, R_{2, \mathrm{CS}}\right) \in \mathcal{R}_{\mathrm{P}}\left(\left(P_{1}-P_{1}^{\prime}\right),\left(P_{2}-P_{2}^{\prime}\right), N_{\mathrm{CS}}\right)
\end{aligned}
$$

where $N_{\mathrm{CS}} \triangleq\left(N+P_{1}^{\prime}+P_{2}^{\prime}+2 \sqrt{P_{1}^{\prime} P_{2}^{\prime}} \rho_{1} \rho_{2}\right)$.

Proof: The rate region is achieved by modifying the rate-splitting scheme for noisy feedback in Section IV-D2 as described in Section V-D. Here, the version of the scheme in Section IV-D2 is chosen where Transmitter 2 decodes the submessages encoded with the concatenated scheme before decoding the submessages encoded with Carleial's Cover-Leung scheme. The analysis of the rate-splitting scheme is based on a genie-aided argument as in [14] and [24]. The details are omitted.
Proposition V.11 (Rate-Splitting for Perfect Partial Feedback II): The capacity region $\mathrm{C}_{\text {PerfectPartialFB }}\left(P_{1}, P_{2}, N\right)$ of the two-user AWGN MAC with perfect partial feedback to Transmitter 2 contains all rate pairs $\left(R_{1}, R_{2}\right)$ which for nonnegative ( $\left.R_{1, \mathrm{ICL}, 1}, R_{1, \mathrm{ICL}, 2}, R_{1, \mathrm{ICS}}\right)$ summing to $R_{1}$; nonnegative ( $\left.R_{2, \mathrm{ICL}, 1}, R_{2, \mathrm{ICL}, 2}, R_{2, \mathrm{ICS}}\right)$ summing to $R_{2}$; and for some choice of $\rho_{1}, \rho_{2} \in[0,1]$ and $P_{1}^{\prime} \in\left[0, P_{1}\right], P_{2}^{\prime} \in\left[0, P_{2}\right]$ satisfy all the 11 constraints $(96 \mathrm{a})-(96 \mathrm{k})$, shown at the bottom of the page, where

$$
\begin{aligned}
N_{1} \triangleq & P_{1}-P_{1}^{\prime}+P_{2}-P_{2}^{\prime}, \\
N_{2} \triangleq & \frac{\left(P_{1}-P_{1}^{\prime}\right)\left(P_{2}-P_{2}^{\prime}\right)+N}{\left(P_{1}-P_{1}^{\prime}+P_{2}-P_{2}^{\prime}+N\right)}+\left(P_{2}-P_{2}^{\prime}\right) \\
& +2 \sqrt{\frac{\left(P_{1}-P_{1}^{\prime}\right)^{2}}{\left(P_{1}-P_{1}^{\prime}+N\right)} \frac{\left(P_{2}-P_{2}^{\prime}\right)^{2}}{\left(P_{1}-P_{1}^{\prime}+P_{2}-P_{2}^{\prime}+N\right)}} .
\end{aligned}
$$

Proof: The rate region is achieved by modifying the ratesplitting scheme for noisy feedback in Section IV-D3 so as to apply also for perfect partial feedback (see Section V-D), and by choosing the parameters of the concatenated scheme as $\eta=2$

$$
\begin{aligned}
R_{1, \mathrm{ICS}} & \leq \frac{1}{4} \log \left(1+\frac{2\left(P_{1}-P_{1}^{\prime}\right)}{N}\right) \\
R_{2, \mathrm{ICS}} & \leq \frac{1}{4} \log \left(1+\frac{\left(P_{2}-P_{2}^{\prime}\right)\left(2+\frac{P_{2}-P_{2}^{\prime}}{P_{1}-P_{1}^{\prime}+N}\right)}{N}\right) \\
R_{1, \mathrm{ICS}}+R_{2, \mathrm{ICS}} & \leq \frac{1}{4} \log \left(1+\frac{P_{1}-P_{1}^{\prime}+P_{2}-P_{2}^{\prime}}{N}\right)+\frac{1}{4} \log \left(1+\frac{N_{2}}{N}\right) \\
R_{1, \mathrm{ICL}, 1} & \leq \frac{1}{4} \log \left(1+\frac{\left(1-\rho_{1}^{2}\right) P_{1}^{\prime}}{P_{1}^{\prime}+N}\right) \\
R_{1, \mathrm{ICL}, 1} & \leq \frac{1}{4} \log \left(1+\frac{\left(1-\rho_{1}^{2}\right) P_{1}^{\prime}}{N_{1}+N}\right)+\frac{1}{4} \log \left(1+\frac{\left(\sqrt{\rho_{1}^{2} P_{1}^{\prime}}+\sqrt{\rho_{2}^{2} P_{2}^{\prime}}\right)^{2}}{N_{1}+N+\left(1-\rho_{1}^{2}\right) P_{1}^{\prime}+\left(1-\rho_{2}^{2}\right) P_{2}^{\prime}}\right) \\
R_{2, \mathrm{ICL}, 1} & \leq \frac{1}{4} \log \left(1+\frac{\left(1-\rho_{2}^{2}\right) P_{2}^{\prime}}{N_{1}+N}\right) \\
R_{1, \mathrm{ICL}, 1}+R_{2, \mathrm{ICL}, 1} & \leq \frac{1}{4} \log \left(1+\frac{P_{1}^{\prime}+P_{2}^{\prime}+2 \sqrt{P_{1}^{\prime} P_{2}^{\prime} \rho_{1}^{2} \rho_{2}^{2}}}{N_{1}+N}\right) \\
R_{1, \mathrm{ICL}, 2} & \leq \frac{1}{4} \log \left(1+\frac{\left(1-\rho_{1}^{2}\right) P_{1}^{\prime}}{P_{1}^{\prime} \frac{N}{P_{1}^{\prime}+N}+N}\right) \\
R_{1, \mathrm{ICL}, 2} & \leq \frac{1}{4} \log \left(1+\frac{\left(1-\rho_{1}^{2}\right) P_{1}^{\prime}}{N_{2}+N}\right)+\frac{1}{4} \log \left(1+\frac{\left(\sqrt{\rho_{1}^{2} P_{1}^{\prime}}+\sqrt{\rho_{2}^{2} P_{2}^{\prime}}\right)^{2}}{N_{2}+N+\left(1-\rho_{1}^{2}\right) P_{1}^{\prime}+\left(1-\rho_{2}^{2}\right) P_{2}^{\prime}}\right) \\
R_{2, \mathrm{ICL}, 2} & \leq \frac{1}{4} \log \left(1+\frac{\left(1-\rho_{2}^{2}\right) P_{2}^{\prime}}{N_{2}+N}\right) \\
R_{1, \mathrm{ICL}, 2}+R_{2, \mathrm{ICL}, 2} & \leq \frac{1}{4} \log \left(1+\frac{P_{1}^{\prime}+P_{2}^{\prime}+2 \sqrt{P_{1}^{\prime} P_{2}^{\prime} \rho_{1}^{2} \rho_{2}^{2}}}{N_{2}+N}\right)
\end{aligned}
$$


and as described in Remark E.1 in Appendix E. The proof follows by accordingly combining Corollary V.8 and the rate constraints which arise from the decodings in Carleial's variation of the Cover-Leung scheme. Again, a genie-aided argument is used in the analysis. The details are omitted.

Remark V.12: In the case of perfect partial feedback, for all channel parameters $P_{1}, P_{2}, N>0$, the achievable regions by Carleial [2] and Willems et al. [23] (Appendices A and B) correspond to the Cover-Leung region $\mathcal{R}_{\mathrm{CL}}\left(P_{1}, P_{2}, N\right)$ (see, e.g., the explanation in [2, Sec. II-C]). Since irrespective of $P_{1}, P_{2}, N>0$, the Cover-Leung region is contained in the two achievable regions in Propositions V.10 and V.11, we conclude that Propositions V.10 and V.11 include also Carleial's and Willems et al.'s regions for perfect partial feedback.

\section{B. Simple Scheme}

If in the simple scheme for noisy feedback in Section IV-B the parameter $b_{1}$ is restricted to be 0 , then the scheme applies also to noisy partial feedback. In particular, in this case it achieves all nonnegative rate pairs $\left(R_{1}, R_{2}\right)$ that satisfy

$$
\begin{aligned}
& R_{1} \leq \frac{1}{4} \log \left(1+\frac{a_{1,1}^{2}}{N}+\frac{a_{2,2}^{2}}{b_{2}^{2} \sigma_{2}^{2}+N}\right) \\
& R_{2} \leq \frac{1}{4} \log \left(1+\frac{a_{2,1}^{2}}{N}+\frac{a_{2,2}^{2}}{b_{2}^{2} \sigma_{2}^{2}+N}\right) \\
& R_{1}+R_{2} \leq \frac{1}{4} \log \left(1+\frac{a_{1,1}^{2}+a_{2,1}^{2}}{N}+\frac{a_{1,2}^{2}+a_{2,2}^{2}}{b_{2}^{2} \sigma_{2}^{2}+N}\right. \\
&\left.+\frac{\left(a_{1,1} a_{2,2}-a_{2,1} a_{1,2}\right)^{2}}{N\left(b_{2}^{2} \sigma_{2}^{2}+N\right)}\right)
\end{aligned}
$$

for some choice of parameters $a_{1,1}, a_{1,2}, a_{2,1}, a_{2,2}, b_{2}$ satisfying

and

$$
a_{1,1}^{2}+a_{1,2}^{2} \leq 2 P_{1}
$$

$$
a_{2,1}^{2}+\left(a_{2,2}-b_{2} a_{2,1}\right)^{2}+b_{2}^{2}\left(a_{1,1}^{2}+N+\sigma_{2}^{2}\right) \leq 2 P_{2} .
$$

The simple scheme for noisy partial feedback is included as a special case in the concatenated scheme for noisy partial feedback described in the next-following section. However, the simple scheme suffices to prove Corollaries V.5 and V.8 and Theorem V.9.

\section{Concatenated Scheme}

1) Scheme: If in the concatenated scheme for noisy feedback in Section IV-C1 the parameter $B_{1}$ is restricted to be the all-zero matrix, then this scheme applies also to noisy partial feedback. In this case, applying the inner encoders with parameters $\eta, \mathbf{a}_{1}, \mathbf{a}_{2}, \mathrm{~B}_{1}=0, \mathrm{~B}_{2}$, and D induces a "new" MAC $\xi_{1}, \xi_{2} \mapsto\left(\hat{\Xi}_{1}, \hat{\Xi}_{2}\right)$ of channel law

$$
\left(\begin{array}{c}
\hat{\Xi}_{1} \\
\hat{\Xi}_{2}
\end{array}\right)=A_{P}\left(\begin{array}{l}
\xi_{1} \\
\xi_{2}
\end{array}\right)+\mathbf{T}_{P}
$$

where the $2 \times 2$ matrix $A_{P}$ is given by

$$
A_{P}=D\left(I_{\eta}-B_{2}\right)^{-1} A_{r}
$$

where $A_{r}$ is defined as in (11); and where the noise vector $\mathbf{T}_{P}$ is a zero-mean bivariate Gaussian

$$
\mathbf{T}_{\mathrm{P}}=\mathrm{D}\left(\mathrm{I}_{\eta}-\mathrm{B}_{2}\right)^{-1}\left(\mathrm{~B}_{2} \mathbf{W}_{2}+\mathbf{Z}\right) .
$$

Defining the $2 \times \eta$ matrix

$$
\mathrm{C}_{\mathrm{P}} \triangleq \mathrm{D}\left(\mathrm{I}_{\eta}-\mathrm{B}_{2}\right)^{-1}
$$

the channel matrix in (98) and the noise vector in (99) can be expressed as

$$
\begin{aligned}
A_{P} & =C_{P} A_{r}, \\
\mathbf{T}_{P} & =C_{P}\left(B_{2} \mathbf{W}_{2}+\mathbf{Z}\right) .
\end{aligned}
$$

For fixed $\eta$ and $B_{2}$ the mapping (100) from $D$ to $C_{P}$ is one-toone, and thus we can parameterize our concatenated scheme for noisy partial feedback by the parameters $\eta, \mathbf{a}_{1}, \mathbf{a}_{2}, \mathrm{~B}_{2}, \mathrm{C}_{\mathrm{P}}$.

Specializing also the power constraints (19) to the choice $\mathrm{B}_{1}=0$ and to noisy partial feedback we see that only parameters $\eta, \mathbf{a}_{1}, \mathbf{a}_{2}$, and $\mathrm{B}_{2}$ satisfying (93) are allowed.

2) Choice of Parameters: In the following we describe guidelines on how to choose the parameters of the concatenated scheme for noisy partial feedback. The guidelines parallel the guidelines presented in Section IV-C2 for noisy feedback. Similarly, the proofs why some of these guidelines are optimal parallel those in Section IV-C2 and are omitted.

Let $P_{1}, P_{2}, N>0, \sigma_{2}^{2} \geq 0$ be given, and for the purpose of description replace the symbols $\xi_{1}$ and $\xi_{2}$ fed to the inner encoders by the independent standard Gaussians $\Xi_{1}$ and $\Xi_{2}$. We start with the matrix $C_{P}$. Given parameters $\eta, \mathbf{a}_{1}, \mathbf{a}_{2}, \mathrm{~B}_{2}$ the matrix $C_{P}$ should be chosen as $C_{P}=C_{P, L M M S E}$, where

$$
\mathrm{C}_{\mathrm{P}, \mathrm{LMMSE}} \triangleq \mathrm{A}_{\mathrm{r}}^{\top}\left(\mathrm{A}_{\mathrm{r}} \mathrm{A}_{\mathrm{r}}^{\top}+N \mathrm{I}_{\eta}+\sigma_{2}^{2} \mathrm{~B}_{2} \mathrm{~B}_{2}^{\top}\right)^{-1} .
$$

The matrix $C_{P, L M M S E}$ in (103) is called the LMMSE-estimation matrix, since by (97), (101), and (102), choosing $C_{P}=$ $C_{\mathrm{P}, \mathrm{LMMSE}}$ implies:

$$
\left(\begin{array}{c}
\hat{\Xi}_{1} \\
\hat{\Xi}_{2}
\end{array}\right)=\mathrm{E}\left[\left(\begin{array}{c}
\Xi_{1} \\
\Xi_{2}
\end{array}\right) \mid Y_{1}, \ldots, Y_{\eta}\right] .
$$

Choosing $C_{P}=C_{P, \text { LMMSE }}$ is optimal in the sense that the corresponding region $\mathcal{R}_{\mathrm{P}}\left(\sigma_{2}^{2}, N ; \eta, \mathbf{a}_{1}, \mathbf{a}_{2}, \mathrm{~B}_{2}, \mathrm{C}_{\mathrm{P}, \mathrm{LMMSE}}\right)$ contains all regions $\mathcal{R}_{\mathrm{P}}\left(\sigma_{2}^{2}, N ; \mathbf{a}_{1}, \mathbf{a}_{2}, \mathrm{~B}_{2}, \mathrm{C}_{\mathrm{P}}\right)$ corresponding to other choices of the parameter $C_{P}$. Choosing $\mathrm{C}_{\mathrm{P}}=\mathrm{UC}_{\mathrm{P}, \mathrm{LMMSE}}$ for some nonsingular $2 \times 2$ matrix $\mathrm{U}$ is also optimal, and for $\eta=2$ choosing $\mathrm{C}_{\mathrm{P}}$ as any nonsingular matrix is optimal.

We next consider the choice of the parameters $\mathbf{a}_{1}, \mathbf{a}_{2}, \mathrm{~B}_{2}$, and first focus on the special case of perfect partial feedback. For perfect partial feedback the parameters $\mathbf{a}_{1}, \mathbf{a}_{2}, \mathrm{~B}_{2}$ should be chosen so that the inputs produced by Inner Encoder 2 correspond to scaled versions of the LMMSE-estimation errors of 
$\Xi_{2}$ when observing the past feedback outputs. Thus, for $\ell \in$ $\{1, \ldots, n\}$, they should satisfy

$$
X_{2, \ell}=\pi_{2, \ell}\left(\Xi_{2}-\mathrm{E}\left[\Xi_{2} \mid Y_{1}, \ldots, Y_{\ell-1}\right]\right)
$$

for some real numbers $\pi_{2,1}, \ldots, \pi_{2, \eta}$. Otherwise there exists a choice of parameters satisfying (104) that-with an appropriate choice of the matrix $\mathrm{C}_{\mathrm{P}}$-strictly improves on the original choice, i.e., corresponds to a larger region than the original choice.

A similar choice for noisy partial feedback is not optimal, and it seems analytically infeasible to determine the optimal choice of the parameters $\mathbf{a}_{1}, \mathbf{a}_{2}, \mathrm{~B}_{1}, \mathrm{~B}_{2}$. However, it is easily seen that for noisy partial feedback the parameters $\mathbf{a}_{1}, \mathbf{a}_{2}, \mathrm{~B}_{1}, \mathrm{~B}_{2}$ should be chosen so that both power constraints (93a) and (93b) are satisfied with equality; otherwise there exists a choice of parameters satisfying (93a) and (93b) that strictly improves on the original choice.

In Remark E.1 (Appendix E), we present for every $\eta \in \mathbb{N}$ a specific (suboptimal) choice of the parameters $\mathbf{a}_{1}, \mathbf{a}_{2}, \mathrm{~B}_{2}$, and $C_{P}$. For this specific choice, the parameter $C_{P}$ is the LMMSEestimation matrix, the parameters $\eta, \mathbf{a}_{1}, \mathbf{a}_{2}, \mathrm{~B}_{2}$ satisfy the power constraints (93a) and (93b) with equality, and when specialized to perfect partial feedback $\mathbf{a}_{1}, \mathbf{a}_{2}, \mathrm{~B}_{2}$ satisfy (45b). We present the corresponding achievable region for $\eta=2$ and equal powers, i.e., $P_{1}=P_{2}=P$, in Corollary V.5 and for $\eta=2$ and perfect partial feedback in Corollary V.8.

\section{Extensions of Concatenated Scheme}

The schemes in Section IV-D apply also to noisy partial feedback, if the parameter $B_{1}$ is restricted to be the all-zero matrix, and if Carleial's variation of the Cover-Leung scheme is specialized to noisy partial feedback. For more details see Section $\mathrm{H}-\mathrm{A}$ in Appendix $\mathrm{H}$ and Section I-A in Appendix I.

\section{E. Proofs}

1) Proof of Theorem V.6: We distinguish between the case of equal powers and of unequal powers. In the case of equal powers, $P_{1}=P_{2}=P$, we consider the achievable region in Corollary V.5, and notice that, irrespective of $P, N>0$ and $\sigma_{2}^{2} \geq 0$, the RHS of the sum-rate constraint (94c) is smaller than the sum of the RHSs of the single-rate constraints (94a) and (94b). Thus, for equal powers the achievable region in Corollary V.5 is a pentagon (and not a rectangle) and there exist achievable pairs $\left(R_{1}, R_{2}\right)$ of sum-rate equal to the RHS of (94c), which is larger than $\frac{1}{2} \log \left(1+\frac{2 P}{N}\right)$. This concludes the proof in the case of equal powers.

In the case of unequal powers, $P_{1} \neq P_{2}$, we use the following rate-splitting/time-sharing strategy. We assume $P_{1}>P_{2}$; the case $P_{1}<P_{2}$ can analogously be treated. Transmitter 1 splits its message $M_{1}$ into two independent submessages: submessage $M_{1,1}$ of rate $R_{1,1}$ and submessage $M_{1,2}$ of rate $R_{1,2}$. During a fraction of time $\frac{P_{1}-P_{2}}{P_{1}+P_{2}}$ Transmitter 1 sends Message $M_{1,2}$ using an optimal no-feedback scheme of power $\left(P_{1}+P_{2}\right)$ while Transmitter 2 is quiet. During the remaining fraction of time $\frac{2 P_{2}}{P_{1}+P_{2}}$ Transmitters 1 and 2 use equal powers $\frac{P_{1}+P_{2}}{2}$ to send messages $M_{1,1}$ and $M_{2}$ with the concatenated scheme in Section V-C1. Choosing the parameters of the concatenated scheme as proposed in Remark E.1 in Appendix E, by Corollary V.5 (where we replace $P$ by $\left.\frac{P_{1}+P_{2}}{2}\right)$ and by the capacity of a AWGN single-user channel, the described rate-splitting/time-sharing scheme achieves the rate pair $\left(R_{1}=R_{1,1}+R_{1,2}, R_{2}\right)$ where $R_{1,1}, R_{1,2}$, and $R_{2}$ are given by (105a) and (105b), shown at the bottom of the page. The proof of (95) follows then by noting that for every $P_{1}, P_{2}, N>0$ and every $\sigma_{2}^{2} \geq 0$ the rate pair $\left(R_{1}, R_{2}\right)$ has a sum-rate which is strictly larger than $\frac{1}{2} \log \left(1+\frac{P_{1}+P_{2}}{N}\right)$, and therefore lies strictly outside the no-feedback capacity region $\mathrm{C}_{\mathrm{NoFB}}\left(P_{1}, P_{2}, N\right)$.

2) Proof of Theorem V.9: We consider an AWGN MAC with powers $P_{1}=1, P_{2}=5$, noise variance $N=5$, and with perfect partial feedback. We prove the theorem by showing that for this channel the rate point $\left(\bar{R}_{1}, \bar{R}_{2}\right)$ :

$$
\begin{aligned}
& \bar{R}_{1}=\frac{1}{4} \log \left(\frac{7}{5}\right) \\
& \bar{R}_{2}=\frac{1}{4} \log \left(3+\frac{3}{7}+\frac{2}{7} \sqrt{\frac{11}{6}}\right)
\end{aligned}
$$

-which by Corollary V.8 is achievable-lies outside the Cover-Leung region $\mathcal{R}_{\mathrm{CL}}\left(P_{1}, P_{2}, N\right)$. This implies that the capacity region $\mathrm{C}_{\text {PerfectPartialFB }}\left(P_{1}, P_{2}, N\right)$ is strictly larger than the Cover-Leung region $\mathcal{R}_{\mathrm{CL}}\left(P_{1}, P_{2}, N\right)$ for $P_{1}=1$ and $P_{2}=N=5$.

$$
\begin{aligned}
R_{1,1}= & \frac{P_{1}-P_{2}}{2\left(P_{1}+P_{2}\right)} \log \left(1+\frac{P_{1}+P_{2}}{N}\right) \\
R_{2}=R_{1,2}= & \frac{P_{2}}{2\left(P_{1}+P_{2}\right)} \log \left(1+\frac{P_{1}+P_{2}}{N}\right) \\
& +\frac{P_{2}}{4\left(P_{1}+P_{2}\right)} \log \left(1+\left(\sqrt{\left.1+\frac{\frac{P_{1}+P_{2}}{2}\left(\frac{P_{1}+P_{2}}{2}+N+\sigma_{2}^{2}\right)}{\left(\frac{P_{1}+P_{2}}{2}+N+\sigma_{2}^{2}+\frac{P_{1}+P_{2}}{2 N} \sigma_{2}^{2}\right)^{2}}-1\right) \frac{\frac{1}{2}\left(P_{1}+P_{2}\right)^{2}}{\left(P_{1}+P_{2}+N\right)}}\right.\right. \\
& \left.\quad+\frac{\left(\frac{P_{1}+P_{2}}{2}\right)^{2}\left(\frac{P_{1}+P_{2}}{2}+N\right)}{\left(P_{1}+P_{2}+N\right)^{2}\left(\frac{P_{1}+P_{2}}{2}+N+\sigma_{2}^{2}+\frac{P_{1}+P_{2}}{2 N} \sigma_{2}^{2}\right)} \cdot \frac{\left(P_{1}+P_{2}+N+\sigma_{2}^{2}\right)}{\left(P_{1}+P_{2}+N+\sigma_{2}^{2}+\frac{P_{1}+P_{2}}{2 N} \sigma_{2}^{2}\right)}\right)
\end{aligned}
$$


Before starting with the proof, we have a closer look at the region $\mathcal{R}_{\mathrm{CL}}\left(P_{1}, P_{2}, N\right)$ and show the following lemma.

Lemma V.13: For $P_{1}, P_{2}, N>0$ and for every $\rho_{1} \in[0,1)$ which satisfies

$$
\frac{P_{2}}{N} \geq \frac{\rho_{1}^{2}}{1-\rho_{1}^{2}}
$$

the rate point $\left(R_{1}\left(\rho_{1}\right), R_{2}\left(\rho_{1}\right)\right)$ given by

$$
R_{1}\left(\rho_{1}\right)=\frac{1}{2} \log \left(1+\frac{P_{1}\left(1-\rho_{1}^{2}\right)}{N}\right)
$$

and by (108), shown at the bottom of the page, lies on the boundary of $\mathcal{R}_{\mathrm{CL}}\left(P_{1}, P_{2}, N\right)$ in the sense that for every $\epsilon>0$

$$
\left(R_{1}\left(\rho_{1}\right), R_{2}\left(\rho_{1}\right)+\epsilon\right) \notin \mathcal{R}_{\mathrm{CL}}\left(P_{1}, P_{2}, N\right) .
$$

Proof: As a first step we examine Expression (108) and characterize $R_{2}\left(\rho_{1}\right)$ more explicitly. To this end, we consider a fixed $\rho_{1} \in[0,1]$ that satisfies (106). Then, we notice that in the minimization in (108) the first term is strictly decreasing in $\rho_{2} \in[0,1]$ whereas the second term is strictly increasing in $\rho_{2}$. Also, for $\rho_{2}=1$ the first term in the maximization in (108) is smaller than the second term, whereas by Condition (106) for $\rho_{2}=0$ the second term is smaller. Thus, for fixed $\rho_{1} \in[0,1]$ satisfying (106) the maximum in (108) is achieved when both terms are equal, i.e., for $\bar{\rho}_{2}$ given by the unique solution in $[0,1]$ to

$$
\begin{aligned}
& \frac{1}{2} \log \left(1+\frac{P_{2}\left(1-\rho_{2}^{2}\right)}{N}\right) \\
&=\frac{1}{2} \log \left(\frac{P_{1}+P_{2}+2 \sqrt{P_{1} P_{2}} \rho_{1} \rho_{2}+N}{P_{1}\left(1-\rho_{1}^{2}\right)+N}\right) .
\end{aligned}
$$

This implies that the rate pair $\left(R_{1}\left(\rho_{1}\right), R_{2}\left(\rho_{1}\right)\right)$ satisfies all three rate constraints defining the rectangle $\mathcal{R}_{\mathrm{CL}}^{\left(\rho_{1}, \bar{\rho}_{2}\right)}\left(P_{1}, P_{2}, N\right)$ with equality, i.e.,

$$
\begin{aligned}
& R_{1}\left(\rho_{1}\right)=\frac{1}{2} \log \left(1+\frac{P_{1}\left(1-\rho_{1}^{2}\right)}{N}\right), \\
& R_{2}\left(\rho_{1}\right)=\frac{1}{2} \log \left(1+\frac{P_{2}\left(1-\left(\bar{\rho}_{2}\right)^{2}\right)}{N}\right)
\end{aligned}
$$

and

$$
\begin{aligned}
R_{1}\left(\rho_{1}\right)+ & R_{2}\left(\rho_{1}\right) \\
& =\frac{1}{2} \log \left(1+\frac{P_{1}+P_{2}+2 \sqrt{P_{1} P_{2}} \rho_{1} \bar{\rho}_{2}}{N}\right) .
\end{aligned}
$$

Hence, $\left(R_{1}\left(\rho_{1}\right), R_{2}\left(\rho_{1}\right)\right)$ is the dominant corner point of the rectangle $\mathcal{R}_{\mathrm{CL}}^{\left(\rho_{1}, \bar{\rho}_{2}\right)}\left(P_{1}, P_{2}, N\right)$, and for all $\epsilon>0$ the rate point $\left(R_{1}\left(\rho_{1}\right), R_{2}\left(\rho_{1}\right)+\epsilon\right)$ lies outside the rate region $\mathcal{R}_{\mathrm{CL}}^{\left(\rho_{1}, \bar{\rho}_{2}\right)}\left(P_{1}, P_{2}, N\right)$. In the remaining we show that the rate point $\left(R_{1}\left(\rho_{1}\right), R_{2}\left(\rho_{1}\right)\right)$ also lies outside the regions $\mathcal{R}_{\mathrm{CL}}^{\left(\rho_{1}^{\prime}, \rho_{2}^{\prime}\right)}\left(P_{1}, P_{2}, N\right)$ for all $\rho_{1}^{\prime}, \rho_{2}^{\prime} \in[0,1]$ not equal to the pair $\left(\rho_{1}, \bar{\rho}_{2}\right)$, and therefore also $\left(R_{1}\left(\rho_{1}\right), R_{2}\left(\rho_{1}\right)+\epsilon\right)$ lies outside these regions for every $\epsilon>0$. This will then conclude the proof of the lemma. We distinguish the following three cases: 1) $\rho_{1}^{\prime}>\rho_{1}$ and $\rho_{2}^{\prime}$ arbitrary; 2) $\rho_{1}^{\prime} \leq \rho_{1}$ and $\rho_{2}^{\prime}>\bar{\rho}_{2}$; and 3) $\rho_{1}^{\prime} \leq \rho_{1}$ and $\rho_{2}^{\prime}<\bar{\rho}_{2}$. In case 1) the rate point $\left(R_{1}\left(\rho_{1}\right), R_{2}\left(\rho_{1}\right)\right)$ lies outside the region $\mathcal{R}_{\mathrm{CL}}^{\left(\rho_{1}^{\prime}, \rho_{2}^{\prime}\right)}\left(P_{1}, P_{2}, N\right)$ because $R_{1}\left(\rho_{1}\right)$ violates the single-rate constraint, see (10a) and (109a). Similarly, in case 2 ) the rate point lies outside the region $\mathcal{R}_{\mathrm{CL}}^{\left(\rho_{1}^{\prime}, \rho_{2}^{\prime}\right)}\left(P_{1}, P_{2}, N\right)$ because in this case $R_{2}\left(\rho_{1}\right)$ violates the single-rate constraint, see (10b) and (109b). Finally, in case $3)$, the rate point lies outside the region $\mathcal{R}_{\mathrm{CL}}^{\left(\rho_{1}^{\prime}, \rho_{2}^{\prime}\right)}\left(P_{1}, P_{2}, N\right)$ because the product $\rho_{1}^{\prime} \cdot \rho_{2}^{\prime}$ is strictly smaller than the product $\rho_{1} \cdot \bar{\rho}_{2}$, and thus the sum $R_{1}\left(\rho_{1}\right)+R_{2}\left(\rho_{1}\right)$ violates the sum-rate constraint; see (10c) and (109c).

We are now ready to prove that the achievable rate point $\left(\bar{R}_{1}, \bar{R}_{2}\right)$ lies outside the Cover-Leung region $\mathcal{R}_{\mathrm{CL}}\left(P_{1}, P_{2}, N\right)$. To this end, we choose $\rho_{1}=\sqrt{6-\sqrt{35}}$ and notice that it satisfies Condition (106) for $P_{2}=N=5$. Hence, Lemma V.13 applies and the rate point $\left(R_{1}^{\mathcal{B}}, R_{2}^{\mathcal{B}}\right)$,

$$
\begin{aligned}
R_{1}^{\mathcal{B}} \triangleq \frac{1}{2} \log \left(\sqrt{\frac{7}{5}}\right), & \\
R_{2}^{\mathcal{B}} \triangleq \max _{\rho_{2} \in[0,1]} & \left\{\operatorname { m i n } \left\{\frac{1}{2} \log \left(1+\left(1-\rho_{2}^{2}\right)\right),\right.\right. \\
& \left.\left.\frac{1}{2} \log \left(\frac{11+2 \sqrt{5(6-\sqrt{35})} \rho_{2}}{\sqrt{35}}\right)\right\}\right\}
\end{aligned}
$$

lies on the boundary of the Cover-Leung region $\mathcal{R}_{\mathrm{CL}}\left(P_{1}, P_{2}, N\right)$, and in particular for every $\epsilon>0$ the rate point $\left(R_{1}^{\mathcal{B}}, R_{2}^{\mathcal{B}}+\epsilon\right)$ lies strictly outside the Cover-Leung region $\mathcal{R}_{\mathrm{CL}}\left(P_{1}, P_{2}, N\right)$. Since

$$
R_{1}^{\mathcal{B}}=\bar{R}_{1}
$$

in order to show that the rate point $\left(\bar{R}_{1}, \bar{R}_{2}\right)$ lies strictly outside $\mathcal{R}_{\mathrm{CL}}\left(P_{1}, P_{2}, N\right)$ it suffices to show that

$$
R_{2}^{\mathcal{B}}<\bar{R}_{2}
$$

$$
R_{2}\left(\rho_{1}\right)=\max _{\rho_{2} \in[0,1]}\left\{\min \left\{\frac{1}{2} \log \left(1+\frac{P_{2}\left(1-\rho_{2}^{2}\right)}{N}\right), \frac{1}{2} \log \left(\frac{P_{1}+P_{2}+2 \sqrt{P_{1} P_{2}} \rho_{1} \rho_{2}+N}{P_{1}\left(1-\rho_{1}^{2}\right)+N}\right)\right\}\right\}
$$


To prove (111) we could compute $\bar{\rho}_{2}$-the value of $\rho_{2}$ which maximizes (110) - and $R_{2}^{\mathcal{B}}$ and then check Condition (111). However, it is easier — and sufficient - to show that for all $\rho_{2} \in$ $[0,1]$ either

$$
\begin{aligned}
\frac{1}{2} \log \left(1+\frac{P_{2}\left(1-\rho_{2}^{2}\right)}{N}\right) & =\frac{1}{2} \log \left(2-\rho_{2}^{2}\right) \\
& <\bar{R}_{2}
\end{aligned}
$$

or

$$
\begin{aligned}
& \frac{1}{2} \log \left(\frac{P_{1}+P_{2}+2 \sqrt{P_{1} P_{2}} \rho_{1} \rho_{2}+N}{P_{1}\left(1-\rho_{1}^{2}\right)+N}\right) \\
= & \frac{1}{2} \log \left(\frac{11+2 \sqrt{5(6-\sqrt{35})} \rho_{2}}{\sqrt{35}}\right) \\
< & \bar{R}_{2} .
\end{aligned}
$$

To this end, note first that the LHS of (112) is decreasing in $\rho_{2} \in[0,1]$, and therefore for all $\sqrt{\frac{1}{7}} \leq \rho_{2} \leq 1$ it follows that

$$
\frac{1}{2} \log \left(2-\rho_{2}^{2}\right) \leq \frac{1}{4} \log \left(3+\frac{3}{7}+\frac{1}{49}\right)<\bar{R}_{2} .
$$

On the other hand, the LHS of (113) is increasing in $\rho_{2}$, and thus for all $0 \leq \rho_{2} \leq \sqrt{\frac{1}{7}}$ :

$$
\begin{aligned}
& \frac{1}{2} \log \left(\frac{11+2 \sqrt{5(6-\sqrt{35})} \rho_{2}}{\sqrt{35}}\right) \\
& \leq \frac{1}{4} \log \left(3+\frac{3}{7}+\frac{1}{35}+\frac{44 \sqrt{\frac{5}{7}(6-\sqrt{35})}}{35}\right. \\
& \left.+\frac{\frac{4}{7}(30-5 \sqrt{35})}{35}\right) \\
& =\frac{1}{4} \log \left(3+\frac{3}{7}+\frac{2}{7}\left(\frac{1}{10}+\frac{22}{\sqrt{35}} \sqrt{(6-\sqrt{35})}\right.\right. \\
& \left.\left.\quad+\frac{12}{7}-2 \sqrt{\frac{5}{7}}\right)\right) \\
& <\bar{R}_{2}
\end{aligned}
$$

where the inequality follows because

$$
\frac{1}{10}+\frac{22}{\sqrt{35}} \sqrt{(6-\sqrt{35})}+\frac{12}{7}-2 \sqrt{\frac{5}{7}}<\sqrt{\frac{11}{6}} .
$$

This concludes the proof of the theorem.

\section{NOISY FEEDback With RECEIVER Side-INFORMATION}

For the setup with receiver side-information we present a new achievable region (Section VI-A) and a scheme that achieves this region (Section VI-B). The proposed scheme is an extension of the concatenated scheme for noisy feedback in Section IV-C and exploits the side-information at the receiver. The simple scheme in Section IV-B and the extended schemes in Section IV-D can be analogously extended to this setup with receiver side-information. For brevity, we omit the description of these latter extensions.

\section{A. Results}

Definition VI.1: Let $\eta$ be a positive integer; $\mathbf{a}_{1}, \mathbf{a}_{2}$ be $\eta$-dimensional vectors; $\mathrm{B}_{1}, \mathrm{~B}_{2}$ be strictly lower-triangular $\eta \times \eta$ matrices; and $\mathrm{C}_{\mathrm{SI}}$ be a $2 \times \eta$ matrix. Depending on the matrix $\mathrm{C}_{\mathrm{SI}}$ the rate region $\mathcal{R}_{\mathrm{SI}}\left(N, \mathrm{~K}_{W_{1} W_{2}} ; \eta, \mathbf{a}_{1}, \mathbf{a}_{2}, \mathrm{~B}_{1}, \mathrm{~B}_{2}, \mathrm{C}_{\mathrm{SI}}\right)$ is defined as follows.

- If the product $\mathrm{C}_{\mathrm{SI}} \mathrm{C}_{\mathrm{SI}}^{\mathrm{T}}$ is nonsingular, ${ }^{12}$ then $\mathcal{R}_{\mathrm{SI}}\left(N, \sigma_{2}^{2} ; \eta, \mathbf{a}_{1}, \mathbf{a}_{2}, \mathrm{~B}_{2}, \mathrm{C}_{\mathrm{SI}}\right)$ is defined as the set of all rate pairs $\left(R_{1}, R_{2}\right)$ satisfying

$$
\begin{aligned}
R_{1} & \leq \frac{1}{2 \eta} \log \frac{\left|\mathrm{C}_{\mathrm{SI}}\left(\mathbf{a}_{1} \mathbf{a}_{1}^{\top}+N \mathrm{I}_{\eta}\right) \mathrm{C}_{\mathrm{SI}}^{\top}\right|}{N\left|\mathrm{C}_{\mathrm{SI}} \mathrm{C}_{\mathrm{SI}}^{\top}\right|}, \\
R_{2} & \leq \frac{1}{2 \eta} \log \frac{\left|\mathrm{C}_{\mathrm{SI}}\left(\mathbf{a}_{2} \mathbf{a}_{2}^{\top}+N \mathrm{I}_{\eta}\right) \mathrm{C}_{\mathrm{SI}}^{\top}\right|}{N\left|\mathrm{C}_{\mathrm{SI}} \mathrm{C}_{\mathrm{SI}}^{\top}\right|}, \\
R_{1}+R_{2} & \leq \frac{1}{2 \eta} \log \frac{\left|\mathrm{C}_{\mathrm{SI}}\left(\mathrm{A}_{\mathrm{r}} \mathrm{A}_{\mathrm{r}}^{\top}+N \mathrm{I}_{\eta}\right) \mathrm{C}_{\mathrm{SI}}^{\top}\right|}{N\left|\mathrm{C}_{\mathrm{SI}} \mathrm{C}_{\mathrm{SI}}^{\top}\right|}
\end{aligned}
$$

where $A_{r}$ is defined in (11).

- If $\mathrm{C}_{\mathrm{SI}} \mathrm{C}_{\mathrm{SI}}^{\top}$ is singular but $\mathrm{C}_{\mathrm{SI}} \neq 0$, then $\mathcal{R}_{\mathrm{SI}}\left(N, \sigma_{2}^{2} ; \eta, \mathbf{a}_{1}, \mathbf{a}_{2}, \mathrm{~B}_{2}, \mathrm{C}_{\mathrm{SI}}\right)$ is defined as the set of all rate pairs $\left(R_{1}, R_{2}\right)$ satisfying (114) when the $2 \times \eta$ matrix $C_{\mathrm{SI}}$ is replaced by the $\eta$-dimensional row-vector obtained by choosing one of the nonzero rows of $\mathrm{C}_{\mathrm{SI}}$.

- If $\mathrm{C}_{\mathrm{SI}}=0$, then $\mathcal{R}_{\mathrm{SI}}\left(N, \sigma_{2}^{2} ; \eta, \mathbf{a}_{1}, \mathbf{a}_{2}, \mathrm{~B}_{2}, \mathrm{C}_{\mathrm{SI}}\right)$ is defined as the set containing only the origin.

(An alternative formulation of the region $\mathcal{R}_{\mathrm{SI}}\left(N, \mathrm{~K}_{W_{1} W_{2}} ; \eta, \mathbf{a}_{1}, \mathbf{a}_{2}, \mathrm{~B}_{1}, \mathrm{~B}_{2}, \mathrm{C}_{\mathrm{SI}}\right)$ is presented in Section D-C in Appendix D.)

Definition VI.2: Define the region

$$
\begin{aligned}
& \mathcal{R}_{\mathrm{SI}}\left(P_{1}, P_{2}, N, \mathrm{~K}_{W_{1} W_{2}}\right) \\
& \quad \triangleq \operatorname{cl}\left(\bigcup \mathcal{R}_{\mathrm{SI}}\left(N, \mathrm{~K}_{W_{1} W_{2}} ; \eta, \mathbf{a}_{1}, \mathbf{a}_{2}, \mathrm{~B}_{1}, \mathrm{~B}_{2}, \mathrm{C}_{\mathrm{SI}}\right)\right),
\end{aligned}
$$

where the union is over all tuples $\left(\eta, \mathbf{a}_{1}, \mathbf{a}_{2}, \mathrm{~B}_{1}, \mathrm{~B}_{2}, \mathrm{C}_{\mathrm{SI}}\right)$ satisfying the trace constraints (19).

Theorem VI.3 (Noisy Feedback With Receiver Side-Information): The capacity region $\mathrm{C}_{\text {NoisyFBSI }}\left(P_{1}, P_{2}, N, \mathrm{~K}_{W_{1} W_{2}}\right)$ of the two-user AWGN MAC with noisy feedback where the receiver is cognizant of the realization of the feedback-noise sequences contains the rate region $\mathcal{R}_{\mathrm{SI}}\left(P_{1}, P_{2}, N, \mathrm{~K}_{W_{1} W_{2}}\right)$, i.e.,

$$
\mathrm{C}_{\text {NoisyFBSI }}\left(P_{1}, P_{2}, N, \mathrm{~K}_{W_{1} W_{2}}\right) \supseteq \mathcal{R}_{\mathrm{SI}}\left(P_{1}, P_{2}, N, \mathrm{~K}_{W_{1} W_{2}}\right) .
$$

Proof: The achievability result is based on the concatenated scheme in Section VI-B1. It is obtained from Theorem IV.3 by setting $\sigma_{1}^{2}=\sigma_{2}^{2}=0$ in the rate expressions in (18) (but not in the power constraints (19)). The reason why in (18) we may set $\sigma_{1}^{2}=\sigma_{2}^{2}=0$ is because in the scheme in Section VI-B1, prior to the decoding, the receiver subtracts off the influence of the feedback-noise sequences $\left\{W_{1, t}\right\}$ and $\left\{W_{2, t}\right\}$. The details of the proof are omitted.

\footnotetext{
${ }^{12}$ Whenever $\eta \in \mathbb{N}$ is larger than 1 , there is no loss in optimality in restricting attention to matrices $\mathrm{C}_{\mathrm{SI}}$ so that $\mathrm{C}_{\mathrm{SI}} \mathrm{C}_{\mathrm{SI}}^{\mathrm{T}}$ is nonsingular.
} 
Remark VI.4: Evaluating the achievable region $\mathcal{R}_{\mathrm{SI}}\left(P_{1}, P_{2}, N, \mathrm{~K}_{W_{1} W_{2}}\right)$ seems to be difficult even numerically. More easily computable (but possibly smaller) achievable regions are obtained by taking the union on the RHS of (115) only over a subset of the parameters $\eta, \mathbf{a}_{1}, \mathbf{a}_{2}, \mathrm{~B}_{1}, \mathrm{~B}_{2}, \mathrm{C}_{\mathrm{SI}}$ satisfying (19). In Section G-A (Appendix G) we present such a subset of parameters and its corresponding achievable region (Corollary G.2). In Section VI-B2 ahead we present more general guidelines on how to choose the parameters $\eta, \mathbf{a}_{1}, \mathbf{a}_{2}, \mathrm{~B}_{1}, \mathrm{~B}_{2}, \mathrm{C}_{\mathrm{SI}}$ for noisy feedback with receiver side-information.

\section{B. Concatenated Scheme}

1) Scheme: In this section we extend our concatenated scheme to noisy feedback with receiver side-information. We use the same outer code and the same inner encoders as in the setting without side-information. The difference is only in the inner decoder. Thus, when fed the pair of symbols $\left(\xi_{1}, \xi_{2}\right)$, the inner encoders produce, as before, sequences of channel inputs

$$
\mathbf{X}_{\nu}=\mathbf{a}_{\nu} \xi_{\nu}+\mathrm{B}_{\nu} \mathbf{V}_{\nu}, \quad \nu \in\{1,2\}
$$

where $\mathbf{X}_{\nu} \triangleq\left(X_{\nu, 1}, \ldots, X_{\nu, \eta}\right)^{\top}, \mathbf{V}_{\nu} \triangleq\left(V_{\nu, 1}, \ldots, V_{\nu, \eta-1}\right)^{\top}$, and where $\mathbf{a}_{1}, \mathbf{a}_{2}$ are $\eta$-dimensional vectors and $\mathrm{B}_{1}, \mathrm{~B}_{2}$ are strictly lower-triangular $\eta \times \eta$ matrices satisfying the power constraints (19). But, we modify the structure of the inner decoder so that it computes the estimates $\left(\hat{\Xi}_{1}, \hat{\Xi}_{2}\right)$ not only as a function of the output sequence but also of the feedback-noise sequences. Again, we choose a linear mapping, i.e., for $\mathbf{Y} \triangleq\left(Y_{1}, \ldots, Y_{\eta}\right)^{\top}$, $\mathbf{W}_{1} \triangleq\left(W_{1,1}, \ldots, W_{1, \eta}\right)^{\top}$, and $\mathbf{W}_{2} \triangleq\left(W_{2,1}, \ldots, W_{2, \eta}\right)^{\top}$, the inner decoder computes

$$
\left(\begin{array}{c}
\hat{\Xi}_{1} \\
\hat{\Xi}_{2}
\end{array}\right)=\mathrm{D}_{0} \mathbf{Y}+\mathrm{D}_{1} \mathbf{W}_{1}+\mathrm{D}_{2} \mathbf{W}_{2}
$$

for $2 \times \eta$ matrices $D_{0}, D_{1}, D_{2}$ of our choice. Given $\mathbf{a}_{1}, \mathbf{a}_{2}, \mathrm{~B}_{1}, \mathrm{~B}_{2}$, and $\mathrm{D}_{0}$ an optimal choice for the matrices $D_{1}$ and $D_{2}$ subtracts off the contributions to $D_{0} \mathbf{Y}$ that come about from the feedback-noise sequences, i.e., an optimal choice of $D_{1}$ and $D_{2}$ satisfies

$$
\begin{aligned}
& \mathrm{D}_{1}=-\mathrm{D}_{0}\left(\mathrm{I}_{\eta}-\left(\mathrm{B}_{1}+\mathrm{B}_{2}\right)\right)^{-1} \mathrm{~B}_{1}, \\
& \mathrm{D}_{2}=-\mathrm{D}_{0}\left(\mathrm{I}_{\eta}-\left(\mathrm{B}_{1}+\mathrm{B}_{2}\right)\right)^{-1} \mathrm{~B}_{2} .
\end{aligned}
$$

Such a choice leads to the following description of the "new" MAC $\xi_{1}, \xi_{2} \mapsto\left(\hat{\Xi}_{1}, \hat{\Xi}_{2}\right)$ :

$$
\left(\begin{array}{c}
\hat{\Xi}_{1} \\
\hat{\Xi}_{2}
\end{array}\right)=\mathrm{A}_{\mathrm{SI}}\left(\begin{array}{l}
\xi_{1} \\
\xi_{2}
\end{array}\right)+\mathbf{T}_{\mathrm{SI}}
$$

where the $2 \times 2$ matrix $A$ is given by

$$
\mathrm{A}_{\mathrm{SI}}=\mathrm{D}_{0}\left(\mathrm{I}_{\eta}-\left(\mathrm{B}_{1}+\mathrm{B}_{2}\right)\right)^{-1} \mathrm{~A}_{\mathrm{r}}
$$

where $A_{r}$ is defined as in (11), and where the noise vector $\mathbf{T}$ is a zero-mean bivariate Gaussian

$$
\mathbf{T}_{\mathrm{SI}}=\mathrm{D}_{0}\left(\mathrm{I}_{\eta}-\left(\mathrm{B}_{1}+\mathrm{B}_{2}\right)\right)^{-1} \mathbf{Z} .
$$

In the following, we shall always assume that $D_{1}$ and $D_{2}$ are optimally chosen so that the "new" MAC is given by (118)-(120). We define the $2 \times \eta$ matrix

$$
\mathrm{C}_{\mathrm{SI}} \triangleq \mathrm{D}_{0}\left(\mathrm{I}_{\eta}-\left(\mathrm{B}_{1}+\mathrm{B}_{2}\right)\right)^{-1}
$$

and hence $A_{S I}$ in (119) and the noise vector $\mathbf{T}_{\mathrm{SI}}$ in (120) can be expressed as

$$
\begin{aligned}
\mathrm{A}_{\mathrm{SI}} & =\mathrm{C}_{\mathrm{SI}} \mathrm{A}_{\mathrm{r}}, \\
\mathbf{T}_{\mathrm{SI}} & =\mathrm{C}_{\mathrm{SI}} \mathbf{Z} .
\end{aligned}
$$

For fixed $\eta, \mathrm{B}_{1}, \mathrm{~B}_{2}$ the mapping (121) from $\mathrm{D}_{0}$, to $\mathrm{C}_{\mathrm{SI}}$ is one-to-one, and thus we can parameterize our concatenated scheme for noisy feedback with receiver side-information by $\eta, \mathbf{a}_{1}, \mathbf{a}_{2}, \mathrm{~B}_{1}, \mathrm{~B}_{2}, \mathrm{C}_{\mathrm{SI}}$.

All parameters $\eta, \mathbf{a}_{1}, \mathbf{a}_{2}, \mathrm{~B}_{1}, \mathrm{~B}_{2}$ that satisfy the power constraints (19) are allowed.

2) Choice of Parameters: As in the previously studied setups we present guidelines on how to choose the parameters $\eta, \mathbf{a}_{1}, \mathbf{a}_{2}, \mathrm{~B}_{1}, \mathrm{~B}_{2}, \mathrm{C}_{\mathrm{SI}}$ of the concatenated scheme. The guidelines parallel the guidelines for noisy feedback and noisy partial feedback in Sections IV-C2 and V-C2; likewise, also the proofs of optimality parallel the proofs in Sections IV-C2 and are omitted.

Let $P_{1}, P_{2}, N>0$ and $\mathrm{K}_{W_{1} W_{2}} \succeq 0$ be given, and for the purpose of describing our guidelines replace the symbols $\xi_{1}, \xi_{2}$ fed to the inner encoders by the independent standard Gaussians $\Xi_{1}, \Xi_{2}$. We first present the optimal choice of the parameter $C_{\mathrm{SI}}$. Given $\eta, \mathbf{a}_{1}, \mathbf{a}_{2}, \mathrm{~B}_{1}, \mathrm{~B}_{2}$ the parameter $\mathrm{C}_{\mathrm{SI}}$ should be chosen as $\mathrm{C}_{\mathrm{SI}}=\mathrm{C}_{\mathrm{SI}, \mathrm{LMMSE}}$, where

$$
\mathrm{C}_{\mathrm{SI}, \mathrm{LMMSE}}=\mathrm{A}_{\mathrm{r}}^{\top}\left(\mathrm{A}_{\mathrm{r}} \mathrm{A}_{\mathrm{r}}^{\top}+N \mathrm{I}_{\eta}\right)^{-1}
$$

since the corresponding achievable region contains all regions corresponding to other choices of the matrix $\mathrm{C}_{\mathrm{SI}}$. The matrix $C_{\text {SI,LMMSE }}$ is called the LMMSE-estimation matrix with side-information, since by (118), (122), and (123) the choice in (124) - combined with the optimal choices of $D_{1}$ and $D_{2}$ defined by (117) and (121) -implies that

$$
\left(\begin{array}{c}
\hat{\Xi}_{1} \\
\hat{\Xi}_{2}
\end{array}\right)=\mathrm{E}\left[\left(\begin{array}{c}
\Xi_{1} \\
\Xi_{2}
\end{array}\right) \mid Y_{1}^{\eta}, W_{1}^{\eta}, W_{2}^{\eta}\right] \text {. }
$$

Obviously, also choosing $\mathrm{C}_{\mathrm{SI}}=\mathrm{U} \mathrm{C}_{\mathrm{SI}, \mathrm{LMMSE}}$ for some nonsingular $2 \times 2$ matrix $\mathrm{U}$ is optimal, and for $\eta=2$ choosing $\mathrm{C}_{\mathrm{SI}}$ as any nonsingular matrix is optimal.

We next consider the choice of parameters $\mathbf{a}_{1}, \mathbf{a}_{2}, \mathrm{~B}_{1}, \mathrm{~B}_{2}$ and focus on the following two special cases:

a) $\eta \in \mathbb{N}$ is arbitrary and $\varrho=1$, i.e., the feedback noises are perfectly correlated;

b) $\eta=2$ and $\varrho \in[-1,1)$ arbitrary.

In these cases, given parameter $\eta \in \mathbb{N}$, the parameters $\mathbf{a}_{1}, \mathbf{a}_{2}, \mathrm{~B}_{1}, \mathrm{~B}_{2}$ should be chosen so that the inner encoders produce

$$
X_{1, \ell}=\pi_{1, \ell}\left(\Xi_{1}-\mathrm{E}\left[\Xi_{1} \mid V_{1}^{\ell-1}\right]\right), \quad \ell \in\{1, \ldots, \eta\},
$$


and

$$
X_{2, \ell}=\pi_{2, \ell}\left(\Xi_{2}-\mathrm{E}\left[\Xi_{2} \mid V_{2}^{\ell-1}\right]\right), \quad \ell \in\{1, \ldots, \eta\}
$$

for some real numbers $\pi_{1,1}, \ldots, \pi_{1, \eta}$ and $\pi_{2,1}, \ldots, \pi_{2, \eta}$. Otherwise, there exists a choice of parameters $\eta, \mathbf{a}_{1}, \mathbf{a}_{2}, \mathrm{~B}_{1}, \mathrm{~B}_{2}, \mathrm{C}_{\mathrm{SI}}$ of the form (125) that strictly improves on the original choice.

In general, it seems difficult to determine the optimal choice of the parameters $\mathbf{a}_{1}, \mathbf{a}_{2}, \mathrm{~B}_{1}, \mathrm{~B}_{2}$. However, it is easily proved that the parameters $\eta, \mathbf{a}_{1}, \mathbf{a}_{2}, \mathrm{~B}_{1}, \mathrm{~B}_{2}, \mathrm{C}_{\mathrm{SI}}$ should be chosen so as to satisfy the power constraints (19a) and (19b) with equality; otherwise there exists a choice of parameters satisfying (19a) and (19b) with equality that strictly improves on the original choice.

In Appendix $\mathrm{G}$, we present a specific choice of the parameters $\mathbf{a}_{1}, \mathbf{a}_{2}, \mathrm{~B}_{1}, \mathrm{~B}_{2}, \mathrm{C}_{\mathrm{SI}}$ that guarantees that $\mathrm{C}_{\mathrm{SI}}$ is the LMMSE-estimation matrix with side-information, the power constraints (19) are satisfied with equality, and $\eta, \mathbf{a}_{1}, \mathbf{a}_{2}, \mathrm{~B}_{1}, \mathrm{~B}_{2}$ satisfy (125) for all $\eta \in \mathbb{N}$ and $\varrho \in[-1,1]$. We present the corresponding achievable region in Corollary G.2 in Appendix G.

\section{SUMMARY}

We have studied four different kinds of two-user AWGN MACs with imperfect feedback:

- noisy feedback, where the feedback links to both transmitters are corrupted by AWGN;

- noisy partial feedback, where one transmitter has noisy feedback and the other no feedback;

- perfect partial feedback, where one transmitter has noisefree feedback and the other no feedback; and

- noisy feedback with receiver side-information, where both transmitters have noisy feedback and the feedback-noise sequences are perfectly known to the receiver.

For each of these settings we have presented a coding scheme (called concatenated scheme) with general parameters, and we have stated the corresponding achievable regions (Theorem IV.3, Theorem V.3, Corollary V.7, and Theorem VI.3). We have improved the concatenated scheme by rate-splitting it either with a simple no-feedback scheme or with Carleial's version of the Cover-Leung scheme. The achievable regions corresponding to these improvements are stated in Proposition IV.13 (noisy feedback) and Propositions V.10 and V.11 (perfect partial feedback).

The two achievable regions for noisy feedback in Theorem IV.3 and Proposition IV.13 exhibit the following three properties. 1) They are monotonically decreasing in the feedback-noise covariance matrix with respect to the Loewner order (Propositions IV.5 and IV.14). 2) They are continuous in the transmit-powers (Propositions IV.5 and IV.14). 3) They converge to Ozarow's perfect-feedback regions when the feedback noise-variances tend to 0 , irrespective of the feedback-noise correlations (Propositions IV.9 and IV.16).

We have further presented guidelines for choosing the parameters of our concatenated schemes (Sections IV-C2, V-C2, and VI-B2), and have suggested (suboptimal) specific choices of the parameters (Sections E-A, F-A, G-A in Appendixes E-G). The achievable regions corresponding to these specific choices are presented in Corollaries IV.6, V.5, V.8, E.3, F.2, Remark F.3, and Corollary G.2.

These achievable regions - combined with the previously described properties of the achievable regions for noisy feedback in Theorem IV.3 and Proposition IV.13-allowed us to infer the following.

1) Feedback - no matter how noisy - is strictly better than no feedback. I.e., irrespective of the feedback-noise variances, the capacity region with one or two noisy feedback links is strictly larger than the no-feedback capacity region (Theorems IV.7 and V.6).

2) The noisy-feedback capacity region converges to the perfect-feedback capacity region as the feedback-noise variances on both links tend to zero-irrespective of the feedback-noise correlations (Theorem IV.17).

3) The Cover-Leung region in general does not equal capacity for perfect partial feedback channels (Theorem V.9). This answers in the negative a question posed by van der Meulen in [18].

\section{APPENDIX A}

\section{CARLEIAL'S REGION}

Carleial proved the achievability result for the AWGN MAC with noisy feedback in Theorem A.2 [2].

Definition A.1: Define the rate region $\mathcal{R}_{\mathrm{Car}}\left(P_{1}, P_{2}, N, \sigma_{1}^{2}, \sigma_{2}^{2}\right)$ as the set of all rate pairs $\left(R_{1}, R_{2}\right)$ which for some nonnegative numbers $R_{1,0}, R_{1,1}$ summing to $R_{1}$, for some nonnegative numbers $R_{2,0}, R_{2,2}$ summing to $R_{2}$, and for some choice of parameters $\alpha_{1}, \alpha_{2}, \beta_{1}, \beta_{2}, v \in[0,1]$ satisfy the 13 conditions (126a)-(126m), shown at the bottom of the next page, where, for $x \in[0,1]$, we define $\bar{x} \triangleq(1-x)$.

Theorem A.2 (Carleial [2]): Consider an AWGN MAC with noisy feedback of transmit powers $P_{1}, P_{2}$, noise variance $N$, and feedback-noise covariance matrix $\mathrm{K}_{W_{1} W_{2}}=\left(\begin{array}{cc}\sigma_{1}^{2} & \varrho \sigma_{1} \sigma_{2} \\ \varrho \sigma_{1} \sigma_{2} & \sigma_{2}^{2}\end{array}\right)$. Irrespective of the noise correlation $\varrho \in[-1,1]$, the region $\mathcal{R}_{\mathrm{Ach}}\left(P_{1}, P_{2}, N, \sigma_{1}^{2}, \sigma_{2}^{2}\right)$ is achievable for this channel, i.e.,

$$
\mathcal{R}_{\mathrm{Ach}}\left(P_{1}, P_{2}, N, \sigma_{1}^{2}, \sigma_{2}^{2}\right) \subseteq \mathrm{C}_{\mathrm{NoisyFB}}\left(P_{1}, P_{2}, N, \mathrm{~K}_{W_{1} W_{2}}\right)
$$

Proposition A.3: The rate region $\mathcal{R}_{\mathrm{Car}}\left(P_{1}, P_{2}, N, \sigma_{1}^{2}, \sigma_{2}^{2}\right)$ collapses to the no-feedback capacity region $\mathrm{C}_{\mathrm{NoFB}}\left(P_{1}, P_{2}, N\right)$ when the feedback-noise variances $\sigma_{1}^{2}, \sigma_{2}^{2}$ exceed a certain threshold depending on the parameters $P_{1}, P_{2}$, and $N$. In particular

$$
\mathcal{R}_{\mathrm{Car}}\left(P_{1}, P_{2}, N, \sigma_{1}^{2}, \sigma_{2}^{2}\right)=\mathrm{C}_{\mathrm{NoFB}}\left(P_{1}, P_{2}, N\right)
$$

for $\sigma_{1}^{2} \geq P_{1}\left(\frac{3}{2}+\frac{P_{2}}{N}\right)$ and $\sigma_{2}^{2} \geq P_{2}\left(\frac{3}{2}+\frac{P_{1}}{N}\right)$.

Proof: For all values of $\sigma_{1}^{2}, \sigma_{2}^{2}$ the region $\mathcal{R}_{\mathrm{Car}}\left(P_{1}, P_{2}, N, \sigma_{1}^{2}, \sigma_{2}^{2}\right)$ trivially contains the no-feedback capacity region $\mathrm{C}_{\mathrm{NoFB}}\left(P_{1}, P_{2}, N\right)$ because the region obtained by substituting $\alpha_{1}=\alpha_{2}=\beta_{1}=\beta_{2}=1$ into (126) coincides with $\mathrm{C}_{\mathrm{NoFB}}\left(P_{1}, P_{2}, N\right)$. Thus, it remains to prove that $\mathcal{R}_{\mathrm{Car}}\left(P_{1}, P_{2}, N, \sigma_{1}^{2}, \sigma_{2}^{2}\right)$ is included in $\mathrm{C}_{\mathrm{NoFB}}\left(P_{1}, P_{2}, N\right)$ 
for all $\sigma_{1}^{2}, \sigma_{2}^{2}$ exceeding some threshold depending on $P_{1}, P_{2}$, and $N$.

To this end, we choose $\sigma_{1}^{2}, \sigma_{2}^{2}>0$ and we fix a rate pair $\left(R_{1}, R_{2}\right)$ in $\mathcal{R}_{\mathrm{Car}}\left(P_{1}, P_{2}, N, \sigma_{1}^{2}, \sigma_{2}^{2}\right)$. We then fix parameters $\alpha_{1}, \alpha_{2}, \beta_{1}, \beta_{2}, v \in[0,1]$, two nonnegative numbers $R_{1,0}$ and $R_{1,1}$ summing to $R_{1}$, and two nonnegative numbers $R_{2,0}$ and $R_{2,2}$ summing to $R_{2}$ so that Constraints (126) are satisfied. We show in the following that if $\sigma_{1}^{2}, \sigma_{2}^{2}>0$ are sufficiently large, then $\left(R_{1}, R_{2}\right)$ lies in $\mathrm{C}_{\mathrm{NoFB}}\left(P_{1}, P_{2}, N\right)$.

By (126a) and (126c) the rate $R_{1}$ satisfies

$$
\begin{aligned}
R_{1} \leq & \frac{1}{2} \log \left(1+\frac{\alpha_{1} \bar{\beta}_{1} P_{1}}{\alpha_{1} \beta_{1} P_{1}+N+\sigma_{2}^{2}}\right) \\
& +\frac{1}{2} \log \left(1+\frac{\alpha_{1} \beta_{1} P_{1}}{N}\right) \\
\leq & \frac{1}{2} \log \left(1+\frac{P_{1}}{N}\right)
\end{aligned}
$$

and by (126b) and (126d), the rate $R_{2}$ satisfies

$$
\begin{aligned}
R_{2} \leq & \frac{1}{2} \log \left(1+\frac{\alpha_{2} \bar{\beta}_{2} P_{2}}{\alpha_{2} \beta_{2} P_{2}+N+\sigma_{1}^{2}}\right) \\
& +\frac{1}{2} \log \left(1+\frac{\alpha_{2} \beta_{2} P_{2}}{N}\right) \\
\leq & \frac{1}{2} \log \left(1+\frac{P_{1}}{N}\right) .
\end{aligned}
$$

Furthermore, by (126a), (126b), and (126h), the sum of the rates $R_{1}+R_{2}$ satisfies (129), shown at the bottom of the next page. Notice that for $\sigma_{1}^{2}, \sigma_{2}^{2}$ larger than some threshold depending on $P_{1}, P_{2}, N$-and in particular for $\sigma_{1}^{2}>P_{1}\left(\frac{3}{2}+\frac{P_{2}}{N}\right)$ and $\sigma_{2}^{2}>P_{2}\left(\frac{3}{2}+\frac{P_{1}}{N}\right)$-irrespective of the chosen parameters $\alpha_{1}, \alpha_{2}, \beta_{1}, \beta_{2}, v$ :

$$
\begin{aligned}
& \frac{N+\alpha_{1} \beta_{1} P_{1}+\alpha_{2} \beta_{2} P_{2}}{\alpha_{1} \beta_{1} P_{1}+N+\sigma_{2}^{2}} \\
& \quad+\frac{\left(\alpha_{1} \beta_{1} P_{1}+\frac{1}{2} N\right)\left(\alpha_{2} \bar{\beta}_{2} P_{2}\right)}{\left(\alpha_{2} \beta_{2} P_{2}+N+\sigma_{1}^{2}\right)\left(\alpha_{1} \beta_{1} P_{1}+N+\sigma_{2}^{2}\right)}<1
\end{aligned}
$$

$$
\begin{aligned}
& R_{1,0} \leq \frac{1}{2} \log \left(1+\frac{\alpha_{1} \bar{\beta}_{1} P_{1}}{\alpha_{1} \beta_{1} P_{1}+N+\sigma_{2}^{2}}\right) \\
& R_{2,0} \leq \frac{1}{2} \log \left(1+\frac{\alpha_{2} \bar{\beta}_{2} P_{2}}{\alpha_{2} \beta_{2} P_{2}+N+\sigma_{1}^{2}}\right) \\
& R_{1,1} \leq \frac{1}{2} \log \left(1+\frac{\alpha_{1} \beta_{1} P_{1}}{N}\right) \\
& R_{2,2} \leq \frac{1}{2} \log \left(1+\frac{\alpha_{2} \beta_{2} P_{2}}{N}\right) \\
& R_{1,0}+R_{20} \leq \frac{1}{2} \log \left(1+\frac{\alpha_{1} \bar{\beta}_{1} P_{1}+\alpha_{2} \bar{\beta}_{2} P_{2}}{N}\right)+\frac{1}{2} \log \left(1+\frac{\bar{\alpha}_{1} P_{1}+\bar{\alpha}_{2} P_{2}+2 \sqrt{\bar{\alpha}_{1} \bar{\alpha}_{2} P_{1} P_{2}}}{N+\alpha_{1} P_{1}+\alpha_{2} P_{2}}\right) \\
& R_{1,0}+R_{2,2} \leq \frac{1}{2} \log \left(1+\frac{\alpha_{1} \bar{\beta}_{1} P_{1}+\alpha_{2} \beta_{2} P_{2}}{N}\right)+\frac{1}{2} \log \left(1+\frac{v\left(\bar{\alpha}_{1} P_{1}+\bar{\alpha}_{2} P_{2}+2 \sqrt{\bar{\alpha}_{1} \bar{\alpha}_{2} P_{1} P_{2}}\right)}{N+\alpha_{1} P_{1}+\alpha_{2} P_{2}}\right) \\
& R_{2,0}+R_{1,1} \leq \frac{1}{2} \log \left(1+\frac{\alpha_{1} \beta_{1} P_{1}+\alpha_{2} \bar{\beta}_{2} P_{2}}{N}\right)+\frac{1}{2} \log \left(1+\frac{\bar{v}\left(\bar{\alpha}_{1} P_{1}+\bar{\alpha}_{2} P_{2}+2 \sqrt{\bar{\alpha}_{1} \bar{\alpha}_{2} P_{1} P_{2}}\right)}{N+\alpha_{1} P_{1}+\alpha_{2} P_{2}}\right) \\
& R_{1,1}+R_{2,2} \leq \frac{1}{2} \log \left(1+\frac{\alpha_{1} \beta_{1} P_{1}+\alpha_{2} \beta_{2} P_{2}}{N}\right) \\
& R_{1}+R_{2,0} \leq \frac{1}{2} \log \left(1+\frac{\alpha_{1} P_{1}+\alpha_{2} \bar{\beta}_{2} P_{2}}{N}\right)+\frac{1}{2} \log \left(1+\frac{\bar{\alpha}_{1} P_{1}+\bar{\alpha}_{2} P_{2}+2 \sqrt{\bar{\alpha}_{1} \bar{\alpha}_{2} P_{1} P_{2}}}{N+\alpha_{1} P_{1}+\alpha_{2} P_{2}}\right) \\
& R_{1,0}+R_{2} \leq \frac{1}{2} \log \left(1+\frac{\alpha_{1} \bar{\beta}_{1} P_{1}+\alpha_{2} P_{2}}{N}\right)+\frac{1}{2} \log \left(1+\frac{\bar{\alpha}_{1} P_{1}+\bar{\alpha}_{2} P_{2}+2 \sqrt{\bar{\alpha}_{1} \bar{\alpha}_{2} P_{1} P_{2}}}{N+\alpha_{1} P_{1}+\alpha_{2} P_{2}}\right) \\
& R_{1}+R_{2,2} \leq \frac{1}{2} \log \left(1+\frac{\alpha_{1} P_{1}+\alpha_{2} \beta_{2} P_{2}}{N}\right)+\frac{1}{2} \log \left(1+\frac{v\left(\bar{\alpha}_{1} P_{1}+\bar{\alpha}_{2} P_{2}+2 \sqrt{\bar{\alpha}_{1} \bar{\alpha}_{2} P_{1} P_{2}}\right)}{N+\alpha_{1} P_{1}+\alpha_{2} P_{2}}\right) \\
& R_{1,1}+R_{2} \leq \frac{1}{2} \log \left(1+\frac{\alpha_{1} \beta_{1} P_{1}+\alpha_{2} P_{2}}{N}\right)+\frac{1}{2} \log \left(1+\frac{\bar{v}\left(\bar{\alpha}_{1} P_{1}+\bar{\alpha}_{2} P_{2}+2 \sqrt{\bar{\alpha}_{1} \bar{\alpha}_{2} P_{1} P_{2}}\right)}{N+\alpha_{1} P_{1}+\alpha_{2} P_{2}}\right) \\
& R_{1}+R_{2} \leq \frac{1}{2} \log \left(1+\frac{P_{1}+P_{2}+2 \sqrt{\bar{\alpha}_{1} \bar{\alpha}_{2} P_{1} P_{2}}}{N}\right)
\end{aligned}
$$


and

$$
\begin{aligned}
& \frac{N+\alpha_{1} \beta_{1} P_{1}+\alpha_{2} \beta_{2} P_{2}}{\alpha_{2} \beta_{2} P_{2}+N+\sigma_{1}^{2}} \\
& \quad+\frac{\left(\alpha_{2} \beta_{2} P_{2}+\frac{1}{2} N\right)\left(\alpha_{1} \bar{\beta}_{1} P_{1}\right)}{\left(\alpha_{1} \beta_{1} P_{1}+N+\sigma_{2}^{2}\right)\left(\alpha_{2} \beta_{2} P_{2}+N+\sigma_{1}^{2}\right)}<1 .
\end{aligned}
$$

Thus, when $\sigma_{1}^{2}, \sigma_{2}^{2}$ exceed a certain threshold depending on $P_{1}, P_{2}$, and $N$, the RHS of (129) is upper bounded by $\frac{1}{2} \log \left(1+\frac{P_{1}+P_{2}}{N}\right)$. We conclude that when $\sigma_{1}^{2}, \sigma_{2}^{2}$ are sufficiently large, then by (127)-(131) the rate pair $\left(R_{1}, R_{2}\right)$ satisfies (5) and hence lies in the no-feedback capacity region $\mathrm{C}_{\mathrm{NoFB}}\left(P_{1}, P_{2}, N\right)$. This concludes the proof.

\section{APPENDIX B}

\section{WILLEMS ET AL.'S REGION}

Willems et al. proved an achievability result for the discrete memoryless MAC with imperfect feedback [23]. The result can easily be extended to the two-user AWGN MAC with noisy feedback (Theorem B.2).

Definition B.1: Define the rate region $\mathcal{R}_{\mathrm{Wil}}\left(P_{1}, P_{2}, N, \sigma_{1}^{2}, \sigma_{2}^{2}\right)$ as the set of all rate pairs $\left(R_{1}, R_{2}\right)$ which for some nonnegative numbers $R_{1,1}$ and $R_{1,2}$ summing to $R_{1}$, for some nonnegative numbers $R_{2,1}$ and $R_{2,2}$ summing to $R_{2}$, and for some parameters $\delta_{1}, \delta_{2}, \rho_{1}, \rho_{2} \in[0,1]$ satisfy the following five constraints:

$$
\begin{aligned}
R_{1,1} & \leq \frac{1}{2} \log \left(1+\frac{\delta_{1} P_{1}}{N}\right) \\
R_{1,0} & \leq \frac{1}{2} \log \left(1+\frac{\bar{\delta}_{1} P_{1}\left(1-\rho_{1}^{2}\right)}{\delta_{1} P_{1}+N+\sigma_{2}^{2}}\right) \\
R_{2,0} & \leq \frac{1}{2} \log \left(1+\frac{\bar{\delta}_{2} P_{2}\left(1-\rho_{2}^{2}\right)}{\delta_{2} P_{2}+N+\sigma_{1}^{2}}\right) \\
R_{2,2} & \leq \frac{1}{2} \log \left(1+\frac{\delta_{2} P_{2}}{N}\right) R_{1,1}+R_{2,2} \\
& \leq \frac{1}{2} \log \left(1+\frac{\delta_{1} P_{1}+\delta_{2} P_{2}}{N}\right) R_{1}+R_{2} \\
& \leq \frac{1}{2} \log \left(1+\frac{P_{1}+P_{2}+2 \sqrt{\bar{\delta}_{1} \bar{\delta}_{2} P_{1} P_{2}} \rho_{1} \rho_{2}}{N}\right) .
\end{aligned}
$$

Theorem B.2 (WillBems et al. [23]): Consider an AWGN MAC with noisy feedback of transmit powers $P_{1}, P_{2}$, noise variance $N$, and feedback-noise covariance matrix $\mathrm{K}_{W_{1} W_{2}}=$ $\left(\begin{array}{cc}\sigma_{1}^{2} & \varrho \sigma_{1} \sigma_{2} \\ \varrho \sigma_{1} \sigma_{2} & \sigma_{2}^{2}\end{array}\right)$. Irrespective of the feedback-noise correlation $\varrho \in[-1,1]$, the region $\mathcal{R}_{\mathrm{Wil}}\left(P_{1}, P_{2}, N, \sigma_{1}^{2}, \sigma_{2}^{2}\right)$ is achievable for this channel, i.e.

$$
\mathcal{R}_{\mathrm{Wil}}\left(P_{1}, P_{2}, N, \sigma_{1}^{2}, \sigma_{2}^{2}\right) \subseteq \mathrm{C}_{\mathrm{NoisyFB}}\left(P_{1}, P_{2}, N, \mathrm{~K}_{W_{1} W_{2}}\right) .
$$

Proposition B.3: The rate region $\mathcal{R}_{\mathrm{Wil}}\left(P_{1}, P_{2}, N, \sigma_{1}^{2}, \sigma_{2}^{2}\right)$ collapses to the no-feedback capacity region $\mathrm{C}_{\mathrm{NoFB}}\left(P_{1}, P_{2}, N\right)$ when the feedback-noise variances $\sigma_{1}^{2}, \sigma_{2}^{2}$ exceed a certain threshold depending on the parameters $P_{1}, P_{2}$, and $N$. In particular:

$$
\mathcal{R}_{\mathrm{Wil}}\left(P_{1}, P_{2}, N, \sigma_{1}^{2}, \sigma_{2}^{2}\right)=\mathrm{C}_{\mathrm{NoFB}}\left(P_{1}, P_{2}, N\right)
$$

for $\sigma_{1}^{2} \geq P_{1}\left(\frac{3}{2}+\frac{P_{2}}{N}\right)$ and $\sigma_{2}^{2} \geq P_{2}\left(\frac{3}{2}+\frac{P_{1}}{N}\right)$.

Proof: Follows along similar lines as the proof of Proposition A.3 in the previous Appendix, and is omitted.

\section{APPENDIX C \\ OPTIMALITY OF LMMSE-ESTIMATION ERROR PARAMETERS FOR PERFECT FEEDBACK}

We show that in our concatenated scheme for perfect feedback it is optimal to choose the parameters $\eta, \mathbf{a}_{1}, \mathbf{a}_{2}, \mathrm{~B}_{1}, \mathrm{~B}_{2}$ so that the two inner encoders produce as their $\ell$ th channel inputs scaled versions of the LMMSE-estimation errors when estimating the fed symbols $\Xi_{1}$ and $\Xi_{2}$ based on the previous outputs $Y_{1}, \ldots, Y_{\ell-1}$; see (45).

Proposition C.1: Assume that $\mathrm{K}_{W_{1} W_{2}}=0$, i.e., perfect feedback. If the parameters $\eta, \mathbf{a}_{1}, \mathbf{a}_{2}, \mathrm{~B}_{1}, \mathrm{~B}_{2}, \mathrm{C}$ satisfy the power constraints (19) but not Conditions (45), then there exist parameters $\eta, \mathbf{a}_{1}^{*}, \mathbf{a}_{2}^{*}, \mathrm{~B}_{1}^{*}, \mathrm{~B}_{2}^{*}, \mathrm{C}^{*}$ satisfying both (19) and (45), and

$\mathcal{R}\left(N, 0 ; \eta, \mathbf{a}_{1}, \mathbf{a}_{2}, \mathrm{~B}_{1}, \mathrm{~B}_{2}, \mathrm{C}\right) \subset \mathcal{R}\left(N, 0 ; \eta, \mathbf{a}_{1}^{*}, \mathbf{a}_{2}^{*}, \mathrm{~B}_{1}^{*}, \mathrm{~B}_{2}^{*}, \mathrm{C}^{*}\right)$

with the inclusion being strict.

The proof is given after the following lemma.

Lemma C.2: Assume that $\mathrm{K}_{W_{1} W_{2}}=0$, i.e., assume perfect feedback. If the parameters $\eta, \mathbf{a}_{1}^{\prime}, \mathbf{a}_{2}^{\prime}, \mathrm{B}_{1}^{\prime}, \mathrm{B}_{2}^{\prime}, \mathrm{C}^{\prime}$ satisfy (19) but violate (45) then there exist parameters $\eta, \hat{\mathbf{a}}_{1}, \hat{\mathbf{a}}_{2}, \hat{\mathrm{B}}_{1}, \hat{\mathrm{B}}_{2}, \hat{\mathrm{C}}$ satisfying the following three conditions:

1) the parameters $\eta, \hat{\mathbf{a}}_{1}, \hat{\mathbf{a}}_{2}, \hat{\mathrm{B}}_{1}, \hat{\mathrm{B}}_{2}, \hat{\mathrm{C}}$ satisfy (45);

2) $\quad \mathcal{R}\left(N, 0 ; \eta, \mathbf{a}_{1}^{\prime}, \mathbf{a}_{2}^{\prime}, \mathrm{B}_{1}^{\prime}, \mathrm{B}_{2}^{\prime}, \mathrm{C}^{\prime}\right)$

$=\mathcal{R}\left(N, 0 ; \eta, \hat{\mathbf{a}}_{1}, \hat{\mathbf{a}}_{2}, \hat{\mathrm{B}}_{1}, \hat{\mathrm{B}}_{2}, \hat{\mathrm{C}}\right) ;$

$$
\begin{aligned}
R_{1}+R_{2} \leq & \frac{1}{2} \log \left(1+\frac{\alpha_{1} \bar{\beta}_{1} P_{1}}{\alpha_{1} \beta_{1} P_{1}+N+\sigma_{2}^{2}}\right)+\frac{1}{2} \log \left(1+\frac{\alpha_{2} \bar{\beta}_{2} P_{2}}{\alpha_{2} \beta_{2} P_{2}+N+\sigma_{1}^{2}}\right)+\frac{1}{2} \log \left(1+\frac{\alpha_{1} \beta_{1} P_{1}+\alpha_{2} \beta_{2} P_{2}}{N}\right) \\
\leq & \frac{1}{2} \log \left(1+\frac{\alpha_{1} \beta_{1} P_{1}+\alpha_{2} \beta_{2} P_{2}}{N}+\frac{\alpha_{1} \bar{\beta}_{1} P_{1}}{N}\left(\frac{N+\alpha_{1} \beta_{1} P_{1}+\alpha_{2} \beta_{2} P_{2}}{\alpha_{1} \beta_{1} P_{1}+N+\sigma_{2}^{2}}+\frac{\left(\alpha_{1} \beta_{1} P_{1}+\frac{1}{2} N\right) \frac{\alpha_{2} \bar{\beta}_{2} P_{2}}{\alpha_{2} \beta_{2} P_{2}+N+\sigma_{1}^{2}}}{\alpha_{1} \beta_{1} P_{1}+N+\sigma_{2}^{2}}\right)\right. \\
& \left.+\frac{\alpha_{2} \bar{\beta}_{2} P_{2}}{N}\left(\frac{N+\alpha_{1} \beta_{1} P_{1}+\alpha_{2} \beta_{2} P_{2}}{\alpha_{2} \beta_{2} P_{2}+N+\sigma_{1}^{2}}+\frac{\left(\alpha_{2} \beta_{2} P_{2}+\frac{1}{2} N\right) \frac{\alpha_{1} \bar{\beta}_{1} P_{1}}{\alpha_{1} \beta_{1} P_{1}+N+\sigma_{2}^{2}}}{\alpha_{2} \beta_{2} P_{2}+N+\sigma_{1}^{2}}\right)\right)
\end{aligned}
$$


3) the parameters $\eta, \hat{\mathbf{a}}_{1}, \hat{\mathbf{a}}_{2}, \hat{\mathrm{B}}_{1}, \hat{\mathrm{B}}_{2}, \hat{\mathrm{C}}$ satisfy (19a) and (19b), and at least one of them with strict inequality.

Proof: Fix parameters $\eta, \mathbf{a}_{1}^{\prime}, \mathbf{a}_{2}^{\prime}, \mathrm{B}_{1}^{\prime}, \mathrm{B}_{2}^{\prime}, \mathrm{C}^{\prime}$ satisfying (19) but violating (45). Define the following new parameters.

- Let $\hat{\mathbf{a}}_{1}=\mathbf{a}_{1}^{\prime}$ and $\hat{\mathbf{a}}_{2}=\mathbf{a}_{2}^{\prime}$.

- Let $\hat{B}_{1}$ and $\hat{B}_{2}$ be so that $\hat{\mathbf{a}}_{1}, \hat{\mathbf{a}}_{2}, \hat{\mathrm{B}}_{1}, \hat{\mathrm{B}}_{2}$ satisfy (45). (Notice that given the parameters $\hat{\mathbf{a}}_{1}$ and $\hat{\mathbf{a}}_{2}$ there exists exactly one choice of the parameters $\hat{\mathrm{B}}_{1}$ and $\hat{\mathrm{B}}_{2}$ satisfying (45). I.e., the scaling coefficients $\left\{\pi_{1, \ell}\right\}_{\ell=1}^{\eta}$ and $\left\{\pi_{2, \ell}\right\}_{\ell=1}^{\eta}$ in (45) are determined by $\hat{\mathbf{a}}_{1}$ and $\hat{\mathbf{a}}_{2}$.)

- Let $\hat{C}=C^{\prime}$.

By construction, our choice $\hat{\mathrm{a}}_{1}, \hat{\mathrm{a}}_{2}, \hat{\mathrm{B}}_{1}, \hat{\mathrm{B}}_{2}, \hat{\mathrm{C}}$ trivially satisfies Condition 1 in the lemma. Moreover, since for $\mathrm{K}_{W_{1} W_{2}}=0$ the region $\mathcal{R}\left(N, 0 ; \eta, \mathbf{a}_{1}, \mathbf{a}_{2}, \mathrm{~B}_{1}, \mathrm{~B}_{2}, \mathrm{C}\right)$ depends only on $\mathbf{a}_{1}, \mathbf{a}_{2}$, and $C$ but not on $B_{1}$ and $B_{2}$ (see Definition IV.1) the regions $\mathcal{R}\left(N, 0 ; \eta, \mathbf{a}_{1}^{\prime}, \mathbf{a}_{2}^{\prime}, \mathrm{B}_{1}^{\prime}, \mathrm{B}_{2}^{\prime}, \mathrm{C}^{\prime}\right)$ and $\mathcal{R}\left(N, 0 ; \eta, \hat{\mathbf{a}}_{1}, \hat{\mathbf{a}}_{2}, \hat{\mathrm{B}}_{1}, \hat{\mathrm{B}}_{2}, \hat{\mathrm{C}}\right)$ coincide. Thus, also Condition 2 is satisfied.

We are left with proving that the parameters $\hat{\mathbf{a}}_{1}, \hat{\mathbf{a}}_{2}, \hat{\mathrm{B}}_{1}, \hat{\mathrm{B}}_{2}, \hat{\mathrm{C}}$ satisfy also Condition 3 . Before doing so, we introduce some helpful assumptions and notation. Assume in the following that Inner Encoder 1 and Inner Encoder 2 are fed the independent standard Gaussians $\Xi_{1}$ and $\Xi_{2}$, respectively. Let $Y_{1}^{\prime}, \ldots, Y_{\eta}^{\prime}$ denote the $\eta$ channel outputs of the original MAC $x_{1}, x_{2} \mapsto Y$ when the inner encoders use the parameters $\eta, \mathbf{a}_{1}^{\prime}, \mathbf{a}_{2}^{\prime}, \mathrm{B}_{1}^{\prime}, \mathrm{B}_{2}^{\prime}, \mathrm{C}^{\prime}$, and similarly, let $\hat{Y}_{1}, \ldots, \hat{Y}_{\eta}$ denote the $\eta$ channel outputs of the original MAC $x_{1}, x_{2} \mapsto Y$ when the inner encoders use the parameters $\eta, \hat{\mathbf{a}}_{1}, \hat{\mathbf{a}}_{2}, \hat{\mathrm{B}}_{1}, \hat{\mathrm{B}}_{2}, \hat{\mathrm{C}}$. Also, let $a_{1, \ell}^{\prime}, a_{2, \ell}^{\prime}, \hat{a}_{1, \ell}$, and $\hat{a}_{2, \ell}$ denote the $\ell$ th entry of the vectors $\mathbf{a}_{1}^{\prime}, \mathbf{a}_{2}^{\prime}, \hat{\mathbf{a}}_{1}$, and $\hat{\mathbf{a}}_{2}$, respectively, and let $b_{1, \ell, j}^{\prime}, b_{2, \ell, j}^{\prime}, \hat{b}_{1, \ell, j}$, and $\hat{b}_{2, \ell, j}$ denote the row- $\ell$ column- $j$ entry of the matrices $\mathrm{B}_{1}^{\prime}, \mathrm{B}_{2}^{\prime}, \hat{\mathrm{B}}_{1}$, and $\hat{\mathrm{B}}_{2}$, respectively, for $j, \ell \in\{1, \ldots, \eta\}$ and $\nu \in\{1,2\}$.

Fix an $\ell \in\{1, \ldots, \eta\}$. By the definition of LMMSE-estimation errors, for all $\nu \in\{1,2\}$ and all real numbers $\left\{b_{\nu, \ell, j}\right\}_{j=1}^{\ell-1}$

$\operatorname{Var}\left(\hat{a}_{\nu, \ell} \Xi_{\nu}-\sum_{j=1}^{\ell-1} b_{\nu, \ell, j} \hat{Y}_{j}\right) \geq \operatorname{Var}\left(\hat{a}_{\nu, \ell} \Xi_{\nu}-\sum_{j=1}^{\ell-1} \hat{b}_{\nu, \ell, j} \hat{Y}_{j}\right)$

with equality if, and only if, $b_{\nu, \ell, j}=\hat{b}_{\nu, \ell, j}$ for all $j \in\{1, \ldots, \ell-$ $1\}$. We would like to prove a similar inequality to (132) but where in the RHS of (132) the outputs $\left\{\hat{Y}_{1}, \ldots, \hat{Y}_{\ell-1}\right\}$ are replaced by the outputs $\left\{Y_{1}^{\prime}, \ldots, Y_{\ell-1}^{\prime}\right\}$. To this end, we notice that since $\hat{\mathbf{a}}_{1}=\mathbf{a}_{1}^{\prime}$ and $\hat{\mathbf{a}}_{2}=\mathbf{a}_{2}^{\prime}$ there exist real numbers $\left\{b_{1, \ell, j}\right\}_{j=1}^{\ell-1}$ and $\left\{b_{2, \ell, j}\right\}_{j=1}^{\ell-1}$ such that

$$
\left(\hat{a}_{\nu, \ell} \Xi_{\nu}-\sum_{j=1}^{\ell-1} b_{\nu, \ell, j} \hat{Y}_{j}\right)=\left(a_{\nu, \ell}^{\prime} \Xi_{\nu}-\sum_{j=1}^{\ell-1} b_{\nu, \ell, j}^{\prime} Y_{j}^{\prime}\right)
$$

with probability 1 . Combining this observation with Inequality (132) the desired inequality follows:

$\operatorname{Var}\left(a_{\nu, \ell}^{\prime} \Xi_{\nu}-\sum_{j=1}^{\ell-1} b_{\nu, \ell, j}^{\prime} Y_{j}^{\prime}\right) \geq \operatorname{Var}\left(\hat{a}_{\nu, \ell} \Xi_{\nu}-\sum_{j=1}^{\ell-1} \hat{b}_{\nu, \ell, j} \hat{Y}_{j}\right)$ with equality if, and only if

$$
\left(a_{\nu, \ell}^{\prime} \Xi_{\nu}-\sum_{j=1}^{\ell-1} b_{\nu, \ell, j}^{\prime} Y_{j}^{\prime}\right)=\left(\hat{a}_{\nu, \ell} \Xi_{1}-\sum_{j=1}^{\ell-1} \hat{b}_{\nu, \ell, j} \hat{Y}_{j}\right)
$$

with probability 1. By (133) and since the parameters $\eta, \mathbf{a}_{1}^{\prime}, \mathbf{a}_{2}^{\prime}, \mathrm{B}_{1}^{\prime}, \mathrm{B}_{2}^{\prime}$ satisfy the power constraints (19), it further follows that also the parameters $\eta, \hat{\mathbf{a}}_{1}, \hat{\mathrm{a}}_{2}, \hat{\mathrm{B}}_{1}, \hat{\mathrm{B}}_{2}$ satisfy Constraints (19). Moreover, since the pairs $\left(\mathrm{B}_{1}^{\prime}, \mathrm{B}_{2}^{\prime}\right)$ and $\left(\hat{\mathrm{B}}_{1}, \hat{\mathrm{B}}_{2}\right)$ differ, not for all $\ell \in\{1, \ldots, \eta\}$ and all $\nu \in\{1,2\}$ equality (134) can hold and thus the parameters $\eta, \hat{\mathbf{a}}_{1}, \hat{\mathbf{a}}_{2}, \hat{\mathrm{B}}_{1}, \hat{\mathrm{B}}_{2}$ satisfy either (19a) or (19b) with strict inequality. This concludes the proof of the lemma.

Proof of Proposition C.1: The proof uses Lemma C.2 twice. Fix parameters $\eta, \mathbf{a}_{1}, \mathbf{a}_{2}, \mathrm{~B}_{1}, \mathrm{~B}_{2}, \mathrm{C}$ satisfying (19) but violating (45). By Lemma C.2, there exist parameters $\eta, \hat{\mathbf{a}}_{1}, \hat{\mathbf{a}}_{2}, \hat{\mathrm{B}}_{1}, \hat{\mathrm{B}}_{2}, \hat{\mathrm{C}}$ that satisfy (45) and

$$
\begin{aligned}
\mathcal{R}\left(N, \mathrm{~K}_{W_{1} W_{2}} ; \eta, \mathbf{a}_{1}, \mathbf{a}_{2}, \mathrm{~B}_{1}, \mathrm{~B}_{2}, \mathrm{C}\right) \\
=\mathcal{R}\left(N, \mathrm{~K}_{W_{1} W_{2}} ; \eta, \hat{\mathbf{a}}_{1}, \hat{\mathbf{a}}_{2}, \hat{\mathrm{B}}_{1}, \hat{\mathrm{B}}_{2}, \hat{\mathrm{C}}\right)
\end{aligned}
$$

and that satisfy (19a) and (19b), whereby one of them with strict inequality. Further, since the parameters $\eta, \hat{\mathbf{a}}_{1}, \hat{\mathbf{a}}_{2}, \hat{\mathrm{B}}_{1}, \hat{\mathrm{B}}_{2}, \hat{\mathrm{C}}$ satisfy either (19a) or (19b) with strict inequality, there exist parameters $\eta, \check{a}_{1}, \breve{a}_{2}, \breve{B}_{1}, \breve{B}_{2}, \breve{C}$ that satisfy both (19a) and (19b) with equality (but not necessarily (45)) and that correspond to a strictly larger region (see Section IV-C2). Thus, by (135)

$$
\begin{aligned}
\mathcal{R}\left(N, \mathrm{~K}_{W_{1} W_{2}} ; \eta, \mathbf{a}_{1}, \mathbf{a}_{2}, \mathrm{~B}_{1}, \mathrm{~B}_{2}, \mathrm{C}\right) \\
\subset \mathcal{R}\left(N, \mathrm{~K}_{W_{1} W_{2}} ; \eta, \check{\mathbf{a}}_{1}, \check{\mathbf{a}}_{2}, \check{\mathrm{B}}_{1}, \check{\mathrm{B}}_{2}, \check{\mathrm{C}}\right)
\end{aligned}
$$

with the inclusion being strict.

Applying Lemma C.2 again, this time to parameters $\eta$, $\check{\mathbf{a}}_{1}, \check{\mathbf{a}}_{2}, \breve{\mathrm{B}}_{1}, \check{\mathrm{B}}_{2}$, $\check{\mathrm{C}}$, we conclude that there exists a choice of parameters $\eta, \mathbf{a}_{1}^{*}, \mathbf{a}_{2}^{*}, \mathrm{~B}_{1}^{*}, \mathrm{~B}_{2}^{*}, \mathrm{C}^{*}$ satisfying both (19) and (45) and

$$
\begin{aligned}
\mathcal{R}\left(N, \mathrm{~K}_{W_{1} W_{2}} ; \eta, \check{\mathbf{a}}_{1}, \check{\mathbf{a}}_{2}, \check{\mathrm{B}}_{1}, \check{\mathrm{B}}_{2}, \check{\mathrm{C}}\right) \\
\quad=\mathcal{R}\left(N, \mathrm{~K}_{W_{1} W_{2}} ; \eta, \mathbf{a}_{1}^{*}, \mathbf{a}_{2}^{*}, \mathrm{~B}_{1}^{*}, \mathrm{~B}_{2}^{*}, \mathrm{C}^{*}\right) .
\end{aligned}
$$

By (136), this implies

$$
\begin{aligned}
\mathcal{R}\left(N, \mathrm{~K}_{W_{1} W_{2}} ; \eta, \mathbf{a}_{1}, \mathbf{a}_{2},\right. & \left.\mathrm{B}_{1}, \mathrm{~B}_{2}, \mathrm{C}\right) \\
& \subset \mathcal{R}\left(N, \mathrm{~K}_{W_{1} W_{2}} ; \eta, \mathbf{a}_{1}^{*}, \mathbf{a}_{2}^{*}, \mathrm{~B}_{1}^{*}, \mathrm{~B}_{2}^{*}, \mathrm{C}^{*}\right)
\end{aligned}
$$

with the inclusion being strict, which concludes the proof.

\section{APPENDIX D}

\section{ALTERNATIVE Formulation OF ACHIEVABLE REgions}

We derive an alternative formulation of the region achieved by our concatenated scheme $\mathcal{R}\left(N, \mathrm{~K}_{W_{1} W_{2}} ; \eta, \mathbf{a}_{1}, \mathbf{a}_{2}, \mathrm{~B}_{1}, \mathrm{~B}_{2}, \mathrm{C}\right)$ when $\mathrm{C}$ is chosen as the LMMSE-estimation matrix $C_{\text {LMMSE }}$ (as defined in (43)) and $\eta, \mathbf{a}_{1}, \mathbf{a}_{2}, \mathrm{~B}_{1}, \mathrm{~B}_{2}$ are arbitrary. Recall that there is no loss in optimality in restricting attention to the choice $C=C_{\text {LMMSE}}$, see Section IV-C2. Similarly, we derive an alternative formulation for the achievable region $\mathcal{R}_{\mathrm{P}}\left(N, \sigma_{2}^{2} ; \eta, \mathbf{a}_{1}, \mathbf{a}_{2}, \mathrm{~B}_{2}, \mathrm{C}_{\mathrm{P}}\right)$ when $\mathrm{C}_{\mathrm{P}}=\mathrm{C}_{\mathrm{P}, \text { LMMSE }}$ (as 
defined in (103)), and an alternative formulation for the achievable region $\mathcal{R}_{\mathrm{SI}}\left(N, \mathrm{~K}_{W_{1} W_{2}} ; \eta, \mathbf{a}_{1}, \mathbf{a}_{2}, \mathrm{~B}_{1}, \mathrm{~B}_{2}, \mathrm{C}_{\mathrm{SI}}\right)$ when $C_{\text {SI }}=C_{\text {SI,LMMSE }}$ (as defined in (124)). These alternative formulations simplify the description of the achievable regions corresponding to our specific choices of parameters suggested in Appendixes E-G. In particular, for perfect feedback the alternative formulation is useful to describe the achievable region corresponding to the choice of parameters in Appendix F, see Remark F.3. The region in Remark F.3 is used in Section IV-E2 to prove that our concatenated scheme for perfect feedback achieves all points in the interior of Ozarow's region $\mathcal{R}_{\mathrm{Oz}}^{\rho^{*}}\left(P_{1}, P_{2}, N\right)$ (Remark IV.8).

\section{A. Noisy Feedback}

Given parameters $\eta, \mathbf{a}_{1}, \mathbf{a}_{2}, \mathrm{~B}_{1}, \mathrm{~B}_{2}$ and $\mathrm{C}=\mathrm{C}_{\text {LMMSE}}$, we derive an alternative formulation for the region achieved by our concatenated scheme $\mathcal{R}\left(N, \mathrm{~K}_{W_{1} W_{2}} ; \eta, \mathbf{a}_{1}, \mathbf{a}_{2}, \mathrm{~B}_{1}, \mathrm{~B}_{2}, \mathrm{C}\right)$.

To simplify notation, in this section we assume that Inner Encoder 1 and Inner Encoder 2 are fed independent zero-mean unit-variance Gaussian random variables and therefore we denote them by $\Xi_{1}$ and $\Xi_{2}$ instead of $\xi_{1}$ and $\xi_{2}$. The region achieved by our concatenated scheme can then be expressed as the set of all nonnegative rate pairs $\left(R_{1}, R_{2}\right)$ satisfying

$$
\begin{aligned}
R_{1} & \leq \frac{1}{\eta} I\left(\Xi_{1} ; \hat{\Xi}_{1}, \hat{\Xi}_{2} \mid \Xi_{2}\right), \\
R_{2} & \leq \frac{1}{\eta} I\left(\Xi_{2} ; \hat{\Xi}_{1}, \hat{\Xi}_{2} \mid \Xi_{1}\right), \\
R_{1}+R_{2} & \leq \frac{1}{\eta} I\left(\Xi_{1}, \Xi_{2} ; \hat{\Xi}_{1}, \hat{\Xi}_{2}\right)
\end{aligned}
$$

where the conditional law of $\left(\hat{\Xi}_{1}, \hat{\Xi}_{2}\right)$ given $\Xi_{1}=\xi_{1}$ and $\Xi_{2}=$ $\xi_{2}$ is determined by the channel law $\xi_{1}, \xi_{2} \mapsto\left(\hat{\Xi}_{1}, \hat{\Xi}_{2}\right)$ in (37) (Section IV-C1). Notice that since $\mathrm{C}$ is the LMMSE-estimation matrix $C_{\text {LMMSE }}$ in (43) (Section IV-C2), by the Gaussianity of the involved random variables the rate constraints in (137) are equivalent to

$$
\begin{aligned}
R_{1} & \leq \frac{1}{\eta} I\left(\Xi_{1} ; Y_{1}, \ldots, Y_{\eta} \mid \Xi_{2}\right), \\
R_{2} & \leq \frac{1}{\eta} I\left(\Xi_{2} ; Y_{1}, \ldots, Y_{\eta} \mid \Xi_{1}\right), \\
R_{1}+R_{2} & \leq \frac{1}{\eta} I\left(\Xi_{1}, \Xi_{2} ; Y_{1}, \ldots, Y_{\eta}\right)
\end{aligned}
$$

where $Y_{1}, \ldots, Y_{\eta}$ are the $\eta$ channel outputs produced by the original channel $x_{1}, x_{2} \mapsto Y$ when the inner encoders are fed the independent standard Gaussians $\Xi_{1}$ and $\Xi_{2}$.

Denote for each channel use $\ell \in\{1, \ldots, \eta\}$ the receiver's innovation by $I_{\ell}$, i.e.,

$$
I_{\ell} \triangleq Y_{\ell}-\mathrm{E}\left[Y_{\ell} \mid Y^{\ell-1}\right]
$$

and the receiver's LMMSE-estimation errors about the symbols $\Xi_{1}$ and $\Xi_{2}$ by $E_{1, \ell}$ and $E_{2, \ell}$, i.e.,

$$
\begin{aligned}
& E_{1, \ell} \triangleq \Xi_{1}-\mathrm{E}\left[\Xi_{1} \mid Y^{\ell}\right], \\
& E_{2, \ell} \triangleq \Xi_{2}-\mathrm{E}\left[\Xi_{2} \mid Y^{\ell}\right] .
\end{aligned}
$$

Then, notice that there exists a bijective mapping between the innovations $I_{1}, \ldots, I_{\eta}$ and the channel outputs $Y_{1}, \ldots, Y_{\eta}$, and by the Gaussianity of the involved random variables, for each $\ell \in\{1, \ldots, \eta\}$, the tuple $\left(E_{1, \ell}, E_{2, \ell}, I_{\ell}\right)$ is independent of the previous outputs and innovations $\left(Y_{1}, \ldots, Y_{\ell-1}, I_{1}, \ldots, I_{\ell-1}\right)$. By the chain rule of mutual information we can therefore rewrite Constraints (138) as

$$
\begin{aligned}
R_{1} & \leq \frac{1}{\eta} \sum_{\ell=1}^{\eta} I\left(\Xi_{1} ; I_{\ell} \mid \Xi_{2}\right), \\
R_{2} & \leq \frac{1}{\eta} \sum_{\ell=1}^{\eta} I\left(\Xi_{2} ; I_{\ell} \mid \Xi_{1}\right), \\
R_{1}+R_{2} & \leq \frac{1}{\eta} \sum_{\ell=1}^{\eta} I\left(\Xi_{1}, \Xi_{2} ; I_{\ell}\right) .
\end{aligned}
$$

In the following, we give a more explicit description of the innovations $\left\{I_{\ell}\right\}_{\ell=1}^{\eta}$ in terms of the entries of the parameters $\mathbf{a}_{1}, \mathbf{a}_{2}, \mathrm{~B}_{1}, \mathrm{~B}_{2}$. For each $\ell \in\{1, \ldots, \eta\}$, let $a_{\nu, \ell}$ denote the $\ell$ th entry of the vector $\mathbf{a}_{\nu}$ and $b_{\nu, \ell, j}$ denote the row- $\ell$ column- $j$ entry of the matrix $\mathrm{B}_{\nu}$, for $j, \ell \in\{1, \ldots, \eta\}$ and $\nu \in\{1,2\}$. Also, let $\alpha_{1, \ell} \triangleq \operatorname{Var}\left(E_{1, \ell}\right)$ and $\alpha_{2, \ell} \triangleq \operatorname{Var}\left(E_{2, \ell}\right)$ denote the variances of $E_{1, \ell}$ and $E_{2, \ell}$, and $\rho_{\ell} \triangleq \frac{\operatorname{Cov}\left[E_{1, \ell}, E_{2, \ell}\right]}{\sqrt{\alpha_{1, \ell} \alpha_{2, \ell}}}$ their correlation coefficient. We can then write the innovations as

$$
I_{1}=Y_{1}=a_{1,1} \Xi_{1}+a_{2,1} \Xi_{2}+Z_{1}
$$

and for $\ell \in\{2, \ldots, \eta\}$ as

$$
\begin{aligned}
I_{\ell}= & a_{1, \ell} E_{1, \ell-1}+a_{2, \ell} E_{2, \ell-1} \\
& +\left(W_{\ell-1}-\mathrm{E}\left[W_{\ell-1} \mid Y^{\ell-1}\right]\right)+Z_{\ell}, \\
= & \kappa_{1, \ell-1} E_{1, \ell-1}+\kappa_{2, \ell-1} E_{2, \ell-1} \\
& +W_{\perp, \ell-1}+Z_{\ell}
\end{aligned}
$$

where

$$
\begin{aligned}
W_{\ell-1} & \triangleq \sum_{j=1}^{\ell-1} b_{1, \ell, j} W_{1, j}+\sum_{j=1}^{\ell-1} b_{2, \ell, j} W_{2, j}, \\
W_{\perp, \ell-1} & \triangleq W_{\ell-1}-\mathrm{E}\left[W_{\ell-1} \mid E_{1, \ell-1}, E_{2, \ell-1}, Y^{\ell-1}\right] \\
& =W_{\ell-1}-\mathrm{E}\left[W_{\ell-1} \mid E_{1, \ell-1}, E_{2, \ell-1}, I^{\ell-1}\right]
\end{aligned}
$$

and

$$
\begin{aligned}
\kappa_{1, \ell-1} \triangleq & a_{1, \ell}+\frac{\alpha_{2, \ell-1} \operatorname{Cov}\left[E_{1, \ell-1}, W_{\ell-1}\right]}{\left(1-\rho_{\ell-1}^{2}\right) \alpha_{1, \ell-1} \alpha_{2, \ell-1}} \\
& -\frac{\rho_{\ell-1} \sqrt{\alpha_{1, \ell-1} \alpha_{2, \ell-1}} \operatorname{Cov}\left[E_{2, \ell-1}, W_{\ell-1}\right]}{\left(1-\rho_{\ell-1}^{2}\right) \alpha_{1, \ell-1} \alpha_{2, \ell-1}}, \\
\kappa_{2, \ell-1} \triangleq & a_{2, \ell}+\frac{\alpha_{1, \ell-1} \operatorname{Cov}\left[E_{2, \ell-1}, W_{\ell-1}\right]}{\left(1-\rho_{\ell-1}^{2}\right) \alpha_{1, \ell-1} \alpha_{2, \ell-1}} \\
& -\frac{\rho_{\ell-1} \sqrt{\alpha_{1, \ell-1} \alpha_{2, \ell-1}} \operatorname{Cov}\left[E_{1, \ell-1}, W_{\ell-1}\right]}{\left(1-\rho_{\ell-1}^{2}\right) \alpha_{1, \ell-1} \alpha_{2, \ell-1}} .
\end{aligned}
$$


Evaluating the mutual information expressions in (141) for the innovations in (142), we conclude that our concatenated scheme for noisy feedback with parameters $\eta, \mathbf{a}_{1}, \mathbf{a}_{2}, \mathrm{~B}_{1}, \mathrm{~B}_{2}$ and $\mathrm{C}=\mathrm{C}_{\mathrm{LMMSE}}$ achieves all rate pairs $\left(R_{1}, R_{2}\right)$ satisfying (148a)-(148c), shown at the bottom of the page, where we defined $\alpha_{1,0} \triangleq 1, \alpha_{2,0} \triangleq 1, \rho_{0} \triangleq 0, \kappa_{1,0} \triangleq a_{1,1}, \kappa_{2,0} \triangleq a_{2,1}$, $W_{\perp, 0} \triangleq 0$.

We conclude this section with a recursive characterization of the variances $\left\{\alpha_{1, \ell}\right\}_{\ell=1}^{\eta}$ and $\left\{\alpha_{2, \ell}\right\}_{\ell=1}^{\eta}$, and the correlation coefficients $\left\{\rho_{\ell}\right\}_{\ell=1}^{\eta}$. Defining $E_{1,0} \triangleq \Xi_{1}, E_{2,0} \triangleq \Xi_{2}$, we find for $\ell \in\{1, \ldots, \eta\}$ :

$$
\begin{aligned}
& E_{1, \ell}=E_{1, \ell-1}-\frac{\operatorname{Cov}\left[E_{1, \ell-1}, I_{\ell}\right]}{\operatorname{Var}\left(I_{\ell}\right)} I_{\ell}, \\
& E_{2, \ell}=E_{2, \ell-1}-\frac{\operatorname{Cov}\left[E_{2, \ell-1}, I_{\ell}\right]}{\operatorname{Var}\left(I_{\ell}\right)} I_{\ell},
\end{aligned}
$$

and consequently, by (142) the recursive expressions (150)-(152), shown at the bottom of the page.

This alternative formulation is used in Corollaries E. 3 and F.2 in Appendixes $\mathrm{E}$ and $\mathrm{F}$ ahead to describe the regions achieved by our concatenated scheme for noisy feedback with the specific choices of parameters described in Section E-A (Appendix E) and F-A (Appendix F). In particular, it is used to describe the region achieved in the special case of perfect feedback when the parameters are chosen as in Section F-A; see Remark F.3.

\section{B. Noisy Partial Feedback}

The desired alternative formulation of $\mathcal{R}_{\mathrm{P}}\left(N, \sigma_{2}^{2} ; \eta, \mathbf{a}_{1}, \mathbf{a}_{2}, \mathrm{~B}_{1}, \mathrm{~B}_{2}, \mathrm{C}_{\mathrm{P}}\right)$ can be derived along the lines shown in the previous Subsection D-A. We omit the details and only present the result.

Fix a choice of parameters $\eta, \mathbf{a}_{1}, \mathbf{a}_{2}, \mathrm{~B}_{1}, \mathrm{~B}_{2}$ and $C_{P}=C_{P, L M M S E}$. Denote the $\ell$ th entry of the vector $a_{\nu}$ by $a_{\nu, \ell}$ and denote the row- $\ell$ column- $j$ entry of the matrix $\mathrm{B}_{2}$ by $b_{2, \ell, j}$, for $j, \ell \in\{1, \ldots, \eta\}$ and $\nu \in\{1,2\}$. Our concatenated scheme for noisy partial feedback and parameters $\eta, \mathbf{a}_{1}, \mathbf{a}_{2}, \mathrm{~B}_{2}, \mathrm{C}_{\mathrm{P}}=\mathrm{C}_{\mathrm{P}, \mathrm{LMMSE}}$ achieves all rate pairs $\left(R_{1}, R_{2}\right)$ satisfying (153a)-(153b), shown at the bottom of the page, where, recall that $\alpha_{1,0}=1, \alpha_{2,0}=1, \rho_{0}=0$, $\kappa_{1,0}=a_{1,1}, \kappa_{2,0}=a_{2,1}, W_{\perp, 0}=0$, and where $\left\{\alpha_{1, \ell}\right\}_{\ell=1}^{\eta-1}$, $\left\{\alpha_{2, \ell}\right\}_{\ell=1}^{\eta-1},\left\{\rho_{\ell}\right\}_{\ell=1}^{\eta-1},\left\{\kappa_{1, \ell}\right\}_{\ell=1}^{\eta-1},\left\{\kappa_{2, \ell}\right\}_{\ell=1}^{\eta-1}$, and $\left\{W_{\perp, \ell}\right\}_{\ell=1}^{\eta-1}$ are defined by $E_{1,0}=\Xi_{1}, E_{2,0}=\Xi_{2}$, and (142)-(152) (Subsection D-A) except that (143) should be replaced by

$$
W_{\ell-1}=\sum_{j=1}^{\ell-1} b_{2, \ell, j} W_{2, j}
$$

$$
\begin{aligned}
R_{1} & \leq \frac{1}{\eta} \sum_{\ell=1}^{\eta} \frac{1}{2} \log \left(1+\frac{\kappa_{1, \ell-1}^{2} \alpha_{1, \ell-1}\left(1-\rho_{\ell-1}^{2}\right)}{\operatorname{Var}\left(W_{\perp, \ell-1}\right)+N}\right) \\
R_{2} & \leq \frac{1}{\eta} \sum_{\ell=1}^{\eta} \frac{1}{2} \log \left(1+\frac{\kappa_{2, \ell-1}^{2} \alpha_{2, \ell-1}\left(1-\rho_{\ell-1}^{2}\right)}{\operatorname{Var}\left(W_{\perp, \ell-1}\right)+N}\right) \\
R_{1}+R_{2} & \leq \frac{1}{\eta} \sum_{\ell=1}^{\eta} \frac{1}{2} \log \left(1+\frac{\kappa_{1, \ell-1}^{2} \alpha_{1, \ell-1}+\kappa_{2, \ell-1}^{2} \alpha_{2, \ell-1}+2 \kappa_{1, \ell-1} \kappa_{2, \ell-1} \sqrt{\alpha_{1, \ell-1} \alpha_{2, \ell-1}} \rho_{\ell-1}}{\operatorname{Var}\left(W_{\perp, \ell-1}\right)+N}\right) .
\end{aligned}
$$

$$
\begin{aligned}
\alpha_{1, \ell} & =\alpha_{1, \ell-1}\left(\frac{\kappa_{1, \ell-1}^{2} \alpha_{1, \ell-1}+\kappa_{2, \ell-1}^{2} \alpha_{2, \ell-1}+2 \kappa_{1, \ell-1} \kappa_{2, \ell-1} \sqrt{\alpha_{1, \ell-1} \alpha_{2, \ell-1}} \rho_{\ell-1}+\operatorname{Var}\left(W_{\perp, \ell-1}\right)+N}{\kappa_{2, \ell-1}^{2} \alpha_{2, \ell-1}\left(1-\rho_{\ell-1}^{2}\right)+\operatorname{Var}\left(W_{\perp, \ell-1}\right)+N}\right) \\
\alpha_{2, \ell} & =\alpha_{2, \ell-1}\left(\frac{\kappa_{1, \ell-1}^{2} \alpha_{1, \ell-1}+\kappa_{2, \ell-1}^{2} \alpha_{2, \ell-1}+2 \kappa_{1, \ell-1} \kappa_{2, \ell-1} \sqrt{\alpha_{1, \ell-1} \alpha_{2, \ell-1}} \rho_{\ell-1}+\operatorname{Var}\left(W_{\perp, \ell-1}\right)+N}{\kappa_{1, \ell-1}^{2} \alpha_{1, \ell-1}\left(1-\rho_{\ell-1}^{2}\right)+\operatorname{Var}\left(W_{\perp, \ell-1}\right)+N}\right) \\
\rho_{\ell} & =\frac{-\kappa_{1, \ell-1} \kappa_{2, \ell-1} \sqrt{\alpha_{1, \ell-1} \alpha_{2, \ell-1}}\left(1-\rho_{\ell-1}^{2}\right)+\rho_{\ell-1}\left(\operatorname{Var}\left(W_{\perp, \ell-1}\right)+N\right)}{\sqrt{\kappa_{1, \ell-1}^{2} \alpha_{1, \ell-1}\left(1-\rho_{\ell-1}^{2}\right)+\operatorname{Var}\left(W_{\perp, \ell-1}\right)+N} \sqrt{\kappa_{2, \ell-1}^{2} \alpha_{2, \ell-1}\left(1-\rho_{\ell-1}^{2}\right)+\operatorname{Var}\left(W_{\perp, \ell-1}\right)+N}} .
\end{aligned}
$$

$$
R_{1}+R_{2} \leq \frac{1}{\eta} \sum_{\ell=1}^{\eta} \frac{1}{2} \log \left(1+\frac{\kappa_{1, \ell-1}^{2} \alpha_{1, \ell-1}+\kappa_{2, \ell-1}^{2} \alpha_{2, \ell-1}+2 \kappa_{1, \ell-1} \kappa_{2, \ell-1} \sqrt{\alpha_{1, \ell-1} \alpha_{2, \ell-1}} \rho_{\ell-1}}{\operatorname{Var}\left(W_{\perp, \ell-1}\right)+N}\right)
$$




\section{Noisy Feedback With Receiver Side-Information}

We derive an alternative formulation of the rate region achieved by our concatenated scheme $\mathcal{R}_{\mathrm{SI}}\left(N, \mathrm{~K}_{W_{1} W_{2}} ; \eta, \mathbf{a}_{1}, \mathbf{a}_{2}, \mathrm{~B}_{1}, \mathrm{~B}_{2}, \mathrm{C}_{\mathrm{SI}}\right)$ for a fixed choice of parameters $\eta, \mathbf{a}_{1}, \mathbf{a}_{2}, \mathrm{~B}_{1}, \mathrm{~B}_{2}$ and $\mathrm{C}_{\mathrm{SI}}=\mathrm{C}_{\mathrm{SI}, \mathrm{LMMSE}}$. Denote the $\ell$ th entry of the vector $\mathbf{a}_{\nu}$ by $a_{\nu, \ell}$ and denote the row- $\ell$ column- $j$ entry of the matrix $\mathrm{B}_{\nu}$ by $b_{\nu, \ell, j}$, for $j, \ell \in\{1, \ldots, \eta\}$ and $\nu \in\{1,2\}$. The desired alternative formulation of $\mathcal{R}_{\mathrm{SI}}\left(N, \mathrm{~K}_{W_{1} W_{2}} ; \eta, \mathbf{a}_{1}, \mathbf{a}_{2}, \mathrm{~B}_{1}, \mathrm{~B}_{2}, \mathrm{C}_{\mathrm{SI}}\right)$ can be derived along the lines described in Section D-A but with the following two modifications. Instead of being defined as in (140), the LMMSE-estimation errors $E_{1, \ell}$ and $E_{2, \ell}$, for $\ell \in\{1, \ldots, \eta\}$, are defined as

$$
\begin{aligned}
& E_{1, \ell} \triangleq \Xi_{1}-\mathrm{E}\left[\Xi_{1} \mid Y^{\ell}, W_{1}^{\ell-1}, W_{2}^{\ell-1}\right], \\
& E_{2, \ell} \triangleq \Xi_{2}-\mathrm{E}\left[\Xi_{2} \mid Y^{\ell}, W_{1}^{\ell-1}, W_{2}^{\ell-1}\right]
\end{aligned}
$$

and instead of being defined as in (139), the innovation $I_{\ell}$, for $\ell \in\{1, \ldots, \eta\}$, is defined as

$$
I_{\ell} \triangleq Y_{\ell}-\mathrm{E}\left[Y_{\ell} \mid Y^{\ell-1}, W_{1}^{\ell-1}, W_{2}^{\ell-1}\right] .
$$

Notice that by (154) and (155):

$$
I_{\ell}=a_{1, \ell} E_{1, \ell-1}+a_{2, \ell} E_{2, \ell-1}+Z_{\ell}, \quad \ell \in\{1, \ldots, \eta\} .
$$

We omit the details of the derivation and only state the resulting alternative formulation of the region $\mathcal{R}_{\mathrm{SI}}\left(N, \mathrm{~K}_{W_{1} W_{2}} ; \eta, \mathbf{a}_{1}, \mathbf{a}_{2}, \mathrm{~B}_{1}, \mathrm{~B}_{2}, \mathrm{C}_{\mathrm{SI}}\right)$. Our concatenated scheme for noisy feedback with receiver side-information and parameters $\eta, \mathbf{a}_{1}, \mathbf{a}_{2}, \mathrm{~B}_{1}, \mathrm{~B}_{2}, \mathrm{C}_{\mathrm{SI}}$ achieves all rate pairs $\left(R_{1}, R_{2}\right)$ satisfying (156a)-(156c), shown at the bottom of the page, where, recall, that $\alpha_{1,0}=1, \alpha_{2,0}=1, \rho_{0}=0$, and where $\left\{\alpha_{1, \ell}\right\}_{\ell=1}^{\eta},\left\{\alpha_{2, \ell}\right\}_{\ell=1}^{\eta}$ and $\left\{\rho_{\ell}\right\}_{\ell=1}^{\eta}$ are defined through (157)-(159), shown at the bottom of the page.

\section{APPENDIX E \\ CHOICE OF PARAMETERS I}

In Section E-A, we present a specific choice of the parameters $\mathbf{a}_{1}, \mathbf{a}_{2}, \mathrm{~B}_{1}, \mathrm{~B}_{2}, \mathrm{C}$ for given $\eta \in \mathbb{N}$. We treat the noisy-feedback setting and the noisy or perfect partial-feedback setting. We denote our choice for noisy feedback by $\overline{\mathbf{a}}_{1}, \overline{\mathbf{a}}_{2}, \bar{B}_{1}, \bar{B}_{2}, \overline{\mathrm{C}}$ and our choice for partial feedback by $\overline{\mathbf{a}}_{1, \mathrm{P}}, \overline{\mathbf{a}}_{2, \mathrm{P}}, \overline{\mathrm{B}}_{2, \mathrm{P}}, \overline{\mathrm{C}}_{\mathrm{P}}$.

As we shall see, our choices are such that $\bar{C}$ and $\bar{C}_{P}$ are LMMSE-estimation matrices. Thus, the region achieved by our concatenated scheme for noisy feedback with parameters $\eta, \overline{\mathbf{a}}_{1}, \overline{\mathbf{a}}_{2}, \overline{\mathrm{B}}_{1}, \overline{\mathrm{B}}_{2}, \overline{\mathrm{C}}_{\text {is obtained by substituting the parameters }}$ into the RHSs of (148) in Section D-A in Appendix D. The resulting achievable region is presented in Corollary E.3 ahead. Similarly, the region achieved by our concatenated scheme for partial feedback with parameters $\eta, \overline{\mathbf{a}}_{1, \mathrm{P}}, \overline{\mathbf{a}}_{2, \mathrm{P}}, \overline{\mathrm{B}}_{2, \mathrm{P}}, \overline{\mathrm{C}}_{\mathrm{P}}$ is obtained by substituting the parameters into the RHSs of (153a)-(153c) in Section D-B in Appendix D. For brevity we do not present this latter achievable region.

\section{A. Description of Parameters}

Let a positive integer $\eta \in \mathbb{N}$ be given. We first consider the noisy-feedback setting; the partial-feedback setting is treated only shortly in Remark E.1 at the end of this section.

Instead of describing our choice $\overline{\mathbf{a}}_{1}, \overline{\mathbf{a}}_{2}, \bar{B}_{1}, \bar{B}_{2}$, and $\bar{C}$ directly, we will describe how Inner Encoder 1 and Inner Encoder 2 map the fed symbols to the sequences of channel inputs $X_{1,1}, \ldots, X_{1, \eta}$ and $X_{2,1}, \ldots, X_{2, \eta}$. This then determines $\overline{\mathbf{a}}_{1}, \overline{\mathbf{a}}_{2}, \overline{\mathrm{B}}_{1}, \overline{\mathrm{B}}_{2}$. The matrix $\overline{\mathrm{C}}$ is chosen as the LMMSE-estimation matrix. For the purpose of describing our choice we replace the pair of input symbols $\xi_{1}$ and $\xi_{2}$ by the independent standard Gaussians $\Xi_{1}$ and $\Xi_{2}$.

$$
\begin{aligned}
R_{1} & \leq \frac{1}{\eta} \sum_{\ell=1}^{\eta} \frac{1}{2} \log \left(1+\frac{a_{1, \ell}^{2} \alpha_{1, \ell-1}\left(1-\rho_{\ell-1}^{2}\right)}{N}\right) \\
R_{2} & \leq \frac{1}{\eta} \sum_{\ell=1}^{\eta} \frac{1}{2} \log \left(1+\frac{a_{2, \ell}^{2} \alpha_{2, \ell-1}\left(1-\rho_{\ell-1}^{2}\right)}{N}\right) \\
R_{1}+R_{2} & \leq \frac{1}{\eta} \sum_{\ell=1}^{\eta} \frac{1}{2} \log \left(1+\frac{a_{1, \ell}^{2} \alpha_{1, \ell-1}+a_{2, \ell}^{2} \alpha_{2, \ell-1}+2 a_{1, \ell} a_{2, \ell} \rho_{\ell-1} \sqrt{\alpha_{1, \ell-1} \alpha_{2, \ell-1}}}{N}\right)
\end{aligned}
$$

$$
\begin{aligned}
\alpha_{1, \ell} & =\alpha_{1, \ell-1}\left(\frac{a_{1, \ell}^{2} \alpha_{1, \ell-1}+a_{2, \ell}^{2} \alpha_{2, \ell-1}+2 a_{1, \ell} a_{2, \ell} \sqrt{\alpha_{1, \ell-1} \alpha_{2, \ell-1}} \rho_{\ell-1}+N}{a_{2, \ell}^{2} \alpha_{2, \ell-1}\left(1-\rho_{\ell-1}^{2}\right)+N}\right)^{-1} \\
\alpha_{2, \ell} & =\alpha_{2, \ell-1}\left(\frac{a_{1, \ell}^{2} \alpha_{1, \ell-1}+a_{2, \ell}^{2} \alpha_{2, \ell-1}+2 a_{1, \ell} a_{2, \ell} \sqrt{\alpha_{1, \ell-1} \alpha_{2, \ell-1}} \rho_{\ell-1}+N}{a_{1, \ell}^{2} \alpha_{1, \ell-1}\left(1-\rho_{\ell-1}\right)^{2}+N}\right)^{-1} \\
\rho_{\ell} & =\frac{-a_{1, \ell} a_{2, \ell} \sqrt{\alpha_{1, \ell-1} \alpha_{2, \ell-1}}\left(1-\rho_{\ell-1}^{2}\right)+\rho_{\ell-1} N}{\sqrt{a_{1, \ell}^{2} \alpha_{1, \ell-1}\left(1-\rho_{\ell-1}^{2}\right)+N} \sqrt{a_{2, \ell}^{2} \alpha_{2, \ell-1}\left(1-\rho_{\ell-1}^{2}\right)+N}}
\end{aligned}
$$


The inner encoders are chosen so as to produce

$$
\begin{aligned}
& X_{1,1}=\sqrt{P_{1}} \Xi_{1}, \\
& X_{2,1}=\sqrt{P_{2}} \Xi_{2},
\end{aligned}
$$

and for $\ell \in\{2, \ldots, \eta\}$ :

$$
\begin{aligned}
& X_{1, \ell}=\sqrt{\frac{P_{1}}{\beta_{1, \ell-1}}}\left(\Xi_{1}-\gamma_{1, \ell-1}^{\top} V_{1}^{\ell-1}\right), \\
& X_{2, \ell}=(-1)^{\ell-1} \sqrt{\frac{P_{2}}{\beta_{2, \ell-1}}}\left(\Xi_{2}-\gamma_{2, \ell-1}^{\top} \mathrm{M}_{\ell-1} V_{2}^{\ell-1}\right),
\end{aligned}
$$

where for $\ell \in\{1, \ldots, \eta-1\}$

$$
\begin{aligned}
& \mathrm{M}_{\ell} \triangleq \operatorname{diag}\left(1,-1,1, \ldots,(-1)^{\ell-1}\right) \\
& \beta_{1, \ell} \triangleq \operatorname{Var}\left(\Xi_{1}-\boldsymbol{\gamma}_{1, \ell}^{\top} V_{1}^{\ell}\right) \\
& \beta_{2, \ell} \triangleq \operatorname{Var}\left(\Xi_{2}-\boldsymbol{\gamma}_{2, \ell}^{\top} \mathrm{M}_{\ell} V_{2}^{\ell}\right) \\
& \boldsymbol{\gamma}_{1, \ell} \triangleq\left(\left(\sigma_{1}^{2}+\sigma_{2}^{2}-2 \varrho \sigma_{1} \sigma_{2}\right) \frac{P_{1}}{N} \mathrm{I}_{\ell}+\mathrm{K}_{V_{1}^{\ell}}\right)^{-1} \times \mathrm{K}_{V_{1}^{\ell}, \Xi_{1}} \\
& \boldsymbol{\gamma}_{2, \ell} \triangleq\left(\left(\sigma_{1}^{2}+\sigma_{2}^{2}-2 \varrho \sigma_{1} \sigma_{2}\right) \frac{P_{2}}{N} \mathrm{I}_{\ell}+\mathrm{K}_{V_{2}^{\ell}}\right)^{-1} \times \mathrm{K}_{V_{2}^{\ell}, \Xi_{2}}
\end{aligned}
$$

Notice that Inner Encoder 2 modulates its inputs with an alternating sequence of +1 or -1 (which is inspired by the Fourier-MEC scheme in [11]), and it multiplies the noisy feedback vectors by the matrix $M_{\ell-1}$ before further processing it (which accounts for the modulation of past inputs). The presented choice of the inner encoders ensures that the input sequences to the original MAC $x_{1}, x_{2} \mapsto Y$ satisfy the average block-power constraints (4). In particular, with the presented choice all input symbols $X_{1,1}, \ldots, X_{1, \eta}$ have the same expected power $P_{1}$, and all input symbols $X_{2,1}, \ldots, X_{2, \eta}$ have the same expected power $P_{2}$.

This encoding scheme corresponds to the following parameters of the concatenated scheme:

$$
\begin{aligned}
& \overline{\mathbf{a}}_{1} \triangleq\left(\begin{array}{llll}
\sqrt{P_{1}} & \sqrt{\frac{P_{1}}{\beta_{1,1}}} & \cdots & \sqrt{\frac{P_{1}}{\beta_{1, \eta-1}}}
\end{array}\right)^{\top},
\end{aligned}
$$

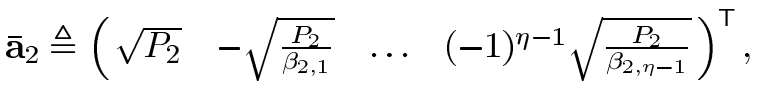

$$
\begin{aligned}
& \text { and } \\
& \overline{\mathrm{B}}_{1} \triangleq\left(\begin{array}{llll}
\mathbf{0} & -\sqrt{\frac{P_{1}}{\beta_{1,1}}} \boldsymbol{\gamma}_{1,1}^{(0)} & \ldots & -\sqrt{\frac{P_{1}}{\beta_{1, \eta-1}}} \boldsymbol{\gamma}_{1, \eta-1}^{(0)}
\end{array}\right)^{\top}, \\
& \overline{\mathrm{B}}_{2} \triangleq\left(\begin{array}{llll}
0 & \sqrt{\frac{P_{2}}{\beta_{2,1}}} \boldsymbol{\gamma}_{2,1}^{(0)} \quad \ldots \quad(-1)^{\eta} \sqrt{\frac{P_{2}}{\beta_{2, \eta-1}}} \boldsymbol{\gamma}_{2, \eta-1}^{(0)}
\end{array}\right)^{\top}
\end{aligned}
$$

where the vectors $\left\{\gamma_{1, \ell}^{(0)}\right\}_{\ell=1}^{\eta-1}$ and $\left\{\boldsymbol{\gamma}_{2, \ell}^{(0)}\right\}_{\ell=1}^{\eta-1}$ are defined as the $\eta$-dimensional vectors obtained by stacking the $\ell$-dimensional column-vector $\gamma_{\nu, \ell}$ on top of an $(\eta-\ell)$-dimensional columnvector with all zero entries, i.e.,

$$
\boldsymbol{\gamma}_{\nu, \ell}^{(0)} \triangleq\left(\begin{array}{c}
\boldsymbol{\gamma}_{\nu, \ell} \\
\mathbf{0}
\end{array}\right)
$$

The parameter $\bar{C}$ is chosen as the LMMSE-estimation matrix $\mathrm{C}_{\text {LMMSE}}$, where recall

$$
\mathrm{C}_{\mathrm{LMMSE}}=\overline{\mathrm{A}}_{\mathrm{r}}^{\top}\left(\overline{\mathrm{A}}_{\mathrm{r}} \overline{\mathrm{A}}_{\mathrm{r}}^{\top}+N \mathrm{I}_{\eta}+\overline{\mathrm{B}}_{\mathrm{r}}\left(\mathrm{K}_{W_{1} W_{2}} \otimes \mathrm{I}_{\eta}\right) \overline{\mathrm{B}}_{\mathrm{r}}^{\top}\right)^{-1}
$$

where $\overline{\mathrm{A}}_{\mathrm{r}} \triangleq\left(\begin{array}{ll}\overline{\mathbf{a}}_{1} & \overline{\mathbf{a}}_{2}\end{array}\right)$ and $\overline{\mathrm{B}}_{\mathrm{r}} \triangleq\left(\begin{array}{ll}\overline{\mathrm{B}}_{1} & \overline{\mathrm{B}}_{2}\end{array}\right)$.

Remark E.1: A similar choice of the parameters can also be made in the case of partial feedback. In this case, we choose the parameters corresponding to the inner encoders and the inner decoder as in (160)-(170) except for replacing (162) by

$$
X_{1, \ell}=\sqrt{P_{1}} \Xi_{1}, \quad \ell \in\{2, \ldots, \eta\}
$$

and replacing (168) by

$$
\gamma_{2, \ell} \triangleq\left(\sigma_{2}^{2} \frac{P_{2}}{N} \mathrm{I}_{\ell}+\mathrm{K}_{V_{2}^{\ell}}\right)^{-1} \mathrm{~K}_{V_{2}^{\ell}, \Xi_{2}} .
$$

We denote the parameters of the concatenated scheme corresponding to this choice by $\overline{\mathbf{a}}_{1, \mathrm{P}}, \overline{\mathbf{a}}_{2, \mathrm{P}}, \overline{\mathrm{B}}_{2, \mathrm{P}}$, and $\overline{\mathrm{C}}_{\mathrm{P}}$.

\section{B. Achievable Region}

We present the achievable region corresponding to our concatenated scheme for noisy feedback with parameters as presented in the previous section.

Definition E.2: For a positive integer $\eta$, define $\overline{\mathcal{R}}_{\eta}\left(P_{1}, P_{2}, N, \mathrm{~K}_{W_{1} W_{2}}\right)$ as the set of all rate-pairs $\left(R_{1}, R_{2}\right)$ satisfying the three rate constraints (171), at the bottom of the next page, where recall that $\alpha_{1,0}=1, \alpha_{2,0}=1, \beta_{1,0}=1$, $\beta_{2,0}=1, \rho_{0}=0, W_{\perp, 0}=0$, where $\left\{\alpha_{1, \ell}\right\}_{\ell=1}^{\eta-1},\left\{\alpha_{2, \ell}\right\}_{\ell=1}^{\eta-1}$, $\left\{\rho_{\ell}\right\}_{\ell=1}^{\eta-1}$ are defined by (172)-(174), shown at the bottom of the next page, and where $\bar{\kappa}_{1,0} \triangleq 1, \bar{\kappa}_{2,0} \triangleq 1$, and $\left\{\bar{\kappa}_{1, \ell}\right\}_{\ell=1}^{\eta-1}$ and $\left\{\bar{\kappa}_{2, \ell}\right\}_{\ell=1}^{\eta-1}$ are defined by ${ }^{13}$

$$
\begin{aligned}
& \bar{\kappa}_{1, \ell} \triangleq 1+\sqrt{\frac{\beta_{1, \ell}}{P_{1}}}\left(\frac{\alpha_{2, \ell} \operatorname{Cov}\left[E_{1, \ell}, W_{\ell}\right]}{\left(1-\rho_{\ell}^{2}\right) \alpha_{1, \ell} \alpha_{2, \ell}}\right. \\
& \left.-\frac{\rho_{\ell} \sqrt{\alpha_{1, \ell} \alpha_{2, \ell}} \operatorname{Cov}\left[E_{2, \ell}, W_{\ell}\right]}{\left(1-\rho_{\ell}^{2}\right) \alpha_{1, \ell} \alpha_{2, \ell}}\right), \\
& \bar{\kappa}_{2, \ell} \triangleq 1+\sqrt{\frac{\beta_{2, \ell}}{P_{2}}}\left(\frac{\alpha_{1, \ell} \operatorname{Cov}\left[E_{2, \ell}, W_{\ell}\right]}{\left(1-\rho_{\ell}^{2}\right) \alpha_{1, \ell} \alpha_{2, \ell}}\right. \\
& \left.-\frac{\rho_{\ell} \sqrt{\alpha_{1, \ell} \alpha_{2, \ell}} \operatorname{Cov}\left[E_{1, \ell}, W_{\ell}\right]}{\left(1-\rho_{\ell}^{2}\right) \alpha_{1, \ell} \alpha_{2, \ell}}\right)
\end{aligned}
$$

and $\left\{\beta_{1, \ell}\right\}_{\ell=1}^{\eta-1},\left\{\beta_{2, \ell}\right\}_{\ell=1}^{\eta-1},\left\{E_{1, \ell}\right\}_{\ell=1}^{\eta-1},\left\{E_{2, \ell}\right\}_{\ell=1}^{\eta-1},\left\{W_{\ell}\right\}_{\ell=1}^{\eta-1}$, and $\left\{W_{\perp, \ell}\right\}_{\ell=1}^{\eta}$ are defined by (160)-(168) and by (140), and (143)-(145).

\footnotetext{
${ }^{13}$ Notice that for each $\nu \in\{1,2\}$ and each $\ell \in\{1, \ldots, \eta-1\}$ we have $\bar{\kappa}_{\nu, \ell}=\sqrt{\frac{\beta_{\nu, \ell}}{P_{\nu}}} \kappa_{\nu, \ell}$, when $\kappa_{\nu, \ell}$ is defined as in (146) or (147) in Section D-A (Appendix D).
} 
Corollary E.3 (Noisy Feedback): The capacity region of the two-user AWGN MAC with noisy feedback contains all rate regions $\overline{\mathcal{R}}_{\eta}\left(P_{1}, P_{2}, N, \mathrm{~K}_{W_{1} W_{2}}\right)$ for positive integers $\eta$, i.e.,

$$
\begin{aligned}
\mathrm{C}_{\text {NoisyFB }}\left(P_{1}, P_{2}, N, \mathrm{~K}_{W_{1} W_{2}}\right) & \\
& \supseteq \mathrm{cl}\left(\bigcup_{\eta \in \mathbb{N}} \overline{\mathcal{R}}_{\eta}\left(P_{1}, P_{2}, N, \mathrm{~K}_{W_{1} W_{2}}\right)\right) .
\end{aligned}
$$

\section{APPENDIX F}

\section{CHOICE OF PARAMETERS II}

In Section F-A we present a second choice of the parameters $\mathbf{a}_{1}, \mathbf{a}_{2}, \mathrm{~B}_{1}, \mathrm{~B}_{2}$, and $\mathrm{C}$ given $\eta \in \mathbb{N}$. We only treat the noisy-feedback setting. The choice we propose is based on extending the choice of parameters in Section E-A with a form of power allocation as suggested in [11]. We denote this choice by $\tilde{\mathbf{a}}_{1}, \tilde{\mathbf{a}}_{2}, \tilde{\mathrm{B}}_{1}, \tilde{\mathrm{B}}_{2}, \tilde{\mathrm{C}}$.

As we shall see, for our choice $\tilde{C}$ is the LMMSE-estimation matrix. Thus, the achievable region of our concatenated scheme with parameters $\eta, \tilde{\mathbf{a}}_{1}, \tilde{\mathbf{a}}_{2}, \tilde{\mathrm{B}}_{1}, \tilde{\mathrm{B}}_{2}, \tilde{\mathrm{C}}$ is obtained by substituting the parameters $\tilde{\mathbf{a}}_{1}, \tilde{\mathbf{a}}_{2}, \tilde{\mathrm{B}}_{1}, \tilde{\mathrm{B}}_{2}$ into the RHSs of (148) in Section D-A. The resulting achievable region is presented in Corollary F. 2.

\section{A. Description of Parameters}

We only consider the noisy feedback setting. An analogous choice of the parameters for the partial feedback setting is obtained by similar modifications as in Remark E.1 in the previous Appendix.

We first describe how Inner Encoder 1 and Inner Encoder 2 map the fed symbols to the sequences of channel inputs $X_{1,1}, \ldots, X_{1, \eta}$ and $X_{2,1}, \ldots, X_{2, \eta}$. This, then determines $\tilde{\mathbf{a}}_{1}, \tilde{\mathbf{a}}_{2}, \tilde{\mathrm{B}}_{1}, \mathrm{~B}_{2}$. The matrix $\tilde{C}$ is chosen as the LMMSE-estimation matrix.

The inner encoders use the same linear strategies as in Section E-A in the previous appendix, with the only difference that here for every fed symbol, Inner Encoder 1 scales the first produced symbol by a constant $\sqrt{r}$, and similarly Inner Encoder 2 scales the first produced symbol by the same constant $\sqrt{r}$, where $r \in$ $[0,1]$ is defined as the solution to

$$
\sqrt{\frac{r^{2} P_{1} P_{2}}{\left(r P_{1}+N\right)\left(r P_{2}+N\right)}}=\rho^{*}\left(P_{1}, P_{2}, N\right) .
$$

$$
\begin{aligned}
R_{1} & \leq \frac{1}{2 \eta} \sum_{\ell=1}^{\eta} \log \left(1+\frac{P_{1} \bar{\kappa}_{1, \ell-1}^{2} \frac{\alpha_{1, \ell-1}}{\beta_{1, \ell-1}}\left(1-\rho_{\ell-1}^{2}\right)}{\operatorname{Var}\left(W_{\perp, \ell-1}\right)+N}\right) \\
R_{2} & \leq \frac{1}{2 \eta} \sum_{\ell=1}^{\eta} \log \left(1+\frac{P_{2} \bar{\kappa}_{2, \ell-1}^{2} \frac{\alpha_{2, \ell-1}}{\beta_{2, \ell-1}}\left(1-\rho_{\ell-1}^{2}\right)}{\operatorname{Var}\left(W_{\perp, \ell-1}\right)+N}\right) \\
R_{1}+R_{2} & \leq \frac{1}{2 \eta} \sum_{\ell=1}^{\eta} \log \left(1+\frac{P_{1} \bar{\kappa}_{1, \ell-1}^{2} \frac{\alpha_{1, \ell-1}}{\beta_{1, \ell-1}}+P_{2} \bar{\kappa}_{2, \ell-1}^{2} \frac{\alpha_{2, \ell-1}}{\beta_{2, \ell-1}}}{\operatorname{Var}\left(W_{\perp, \ell-1}\right)+N}+\frac{2 \sqrt{P_{1} P_{2}} \bar{\kappa}_{1, \ell-1} \bar{\kappa}_{2, \ell-1} \sqrt{\frac{\alpha_{1, \ell-1}}{\beta_{1, \ell-1}}} \sqrt{\frac{\alpha_{2, \ell-1}}{\beta_{2, \ell-1}}} \rho_{\ell-1}}{\operatorname{Var}\left(W_{\perp, \ell-1}\right)+N}\right)
\end{aligned}
$$

$$
\begin{gathered}
\alpha_{1, \ell}=\alpha_{1, \ell-1}\left(\frac{P_{1} \bar{\kappa}_{1, \ell-1}^{2} \frac{\alpha_{1, \ell-1}}{\beta_{1, \ell-1}}+P_{2} \bar{\kappa}_{2, \ell-1}^{2} \frac{\alpha_{2, \ell-1}}{\beta_{2, \ell-1}}}{P_{2} \bar{\kappa}_{2, \ell-1}^{2} \frac{\alpha_{2, \ell-1}}{\beta_{2, \ell-1}}\left(1-\rho_{\ell-1}^{2}\right)+\operatorname{Var}\left(W_{\perp, \ell-1}\right)+N}\right. \\
\left.\quad+\frac{2 \sqrt{P_{1} P_{2}} \bar{\kappa}_{1, \ell-1} \bar{\kappa}_{2, \ell-1} \sqrt{\frac{\alpha_{1, \ell-1} \alpha_{2, \ell-1}}{\beta_{1, \ell-1} \beta_{2, \ell-1}}}(-1)^{\ell} \rho_{\ell-1}+\operatorname{Var}\left(W_{\perp, \ell-1}\right)+N}{P_{2} \bar{\kappa}_{2, \ell-1}^{2} \frac{\alpha_{2, \ell-1}}{\beta_{2, \ell-1}}\left(1-\rho_{\ell-1}^{2}\right)+\operatorname{Var}\left(W_{\perp, \ell-1}\right)+N}\right)^{-1} \\
\alpha_{2, \ell}=\alpha_{2, \ell-1}\left(\frac{P_{1} \bar{\kappa}_{1, \ell-1}^{2} \frac{\alpha_{1, \ell-1}}{\beta_{1, \ell-1}}+P_{2} \bar{\kappa}_{2, \ell-1}^{2} \frac{\alpha_{2, \ell-1}}{\beta_{2, \ell-1}}}{P_{1} \bar{\kappa}_{1, \ell-1}^{2} \frac{\alpha_{1, \ell-1}}{\beta_{1, \ell-1}}\left(1-\rho_{\ell-1}^{2}\right)+\operatorname{Var}\left(W_{\perp, \ell-1}\right)+N}\right. \\
\left.\quad+\frac{2 \sqrt{P_{1} P_{2}} \bar{\kappa}_{1, \ell-1} \bar{\kappa}_{2, \ell-1} \sqrt{\frac{\alpha_{1, \ell-1} \alpha_{2, \ell-1}}{\beta_{1, \ell-1} \beta_{2, \ell-1}}}(-1)^{\ell} \rho_{\ell-1}+\operatorname{Var}\left(W_{\perp, \ell-1}\right)+N}{P_{1} \bar{\kappa}_{1, \ell-1}^{2} \frac{\alpha_{1, \ell-1}}{\beta_{1, \ell-1}}\left(1-\rho_{\ell-1}^{2}\right)+\operatorname{Var}\left(W_{\perp, \ell-1}\right)+N}\right)^{-1} \\
\rho_{\ell}=\rho_{\ell-1} \frac{-\sqrt{P_{1} P_{2}} \bar{\kappa}_{1, \ell-1} \bar{\kappa}_{2, \ell-1} \sqrt{\frac{\alpha_{1, \ell-1}}{\beta_{1, \ell-1}}} \sqrt{\frac{\alpha_{2, \ell-1}}{\beta_{2, \ell-1}}}\left(1-\rho_{\ell-1}^{2}\right)+\rho_{\ell-1}\left(\operatorname{Var}\left(W_{\perp, \ell-1}\right)+N\right)}{\sqrt{P_{1} \bar{\kappa}_{1, \ell-1}^{2} \frac{\alpha_{1, \ell-1}}{\beta_{1, \ell-1}}\left(1-\rho_{\ell-1}^{2}\right)+\operatorname{Var}\left(W_{\perp, \ell-1}\right)+N} \sqrt{P_{2} \bar{\kappa}_{2, \ell-1}^{2} \frac{\alpha_{2, \ell-1}}{\beta_{2, \ell-1}}\left(1-\rho_{\ell-1}^{2}\right)+\operatorname{Var}\left(W_{\perp, \ell-1}\right)+N}}
\end{gathered}
$$


Equation (177) has a unique solution in $[0,1]$ because (177) is strictly increasing in $r \in[0,1]$ and by

$$
0<\rho^{*}\left(P_{1}, P_{2}, N\right)<\sqrt{\frac{P_{1} P_{2}}{\left(P_{1}+N\right)\left(P_{2}+N\right)}} .
$$

Here, (178) holds by the continuity of the expressions in (8), and because for $\rho=0$ the RHS of (8) is strictly larger than its LHS, whereas for $\rho=\sqrt{\frac{P_{1} P_{2}}{\left(P_{1}+N\right)\left(P_{2}+N\right)}}$ the LHS of (8) is strictly larger than its RHS.

The reason for scaling the first produced symbols by $\sqrt{r}<1$ is to ensure that the correlation coefficient $\rho_{1}$ satisfies $\rho_{1}=$ $-\rho^{*}\left(P_{1}, P_{2}, N\right)$. This property is used in the proof of Remark IV.8 in Section IV-E2, where we show that for perfect feedback and with the choice of parameters presented in this section our concatenated scheme achieves the sum-rate capacity.

The trick of reducing the powers of certain channel inputs $X_{1, t}$ and $X_{2, t}$ in order to control the next correlation coefficient $\rho_{t}$ was introduced in Kramer's perfect-feedback scheme [11]. Ozarow uses a different trick in his scheme [13]. He assumes that the two transmitters share a common randomness, which allows them to vary a specific correlation coefficient $\rho_{t}$ by adding a scaled version of the common randomness to their channel inputs $X_{1, t}$ and $X_{2, t}$.

For the detailed description of the inner encoders we again replace the fed symbols $\xi_{1}, \xi_{2}$ by the independent standard Gaussians $\Xi_{1}$ and $\Xi_{2}$. Then, Inner Encoder 1 produces

$$
\begin{aligned}
& X_{1,1}=\sqrt{r P_{1}} \Xi_{1}, \\
& X_{1, \ell}=\sqrt{\frac{P_{1}}{\beta_{1, \ell-1}}}\left(\Xi_{1}-\gamma_{1, \ell-1}^{\top} V_{1}^{\ell-1}\right), \quad \ell \in\{2, \ldots, \eta\},
\end{aligned}
$$

and Inner Encoder 2 produces

$$
\begin{aligned}
& X_{2,1}= \sqrt{r P_{2}} \Xi_{2}, \\
& X_{2, \ell}=(-1)^{\ell-1} \sqrt{\frac{P_{2}}{\beta_{2, \ell-1}}}\left(\Xi_{2}-\gamma_{2, \ell-1}^{\top} \mathrm{M}_{\ell-1} V_{2,1}^{\ell-1}\right), \\
& \quad \ell \in\{2, \ldots, \eta\}
\end{aligned}
$$

where $\left\{\mathrm{M}_{\ell}\right\}_{\ell=1}^{\eta-1},\left\{\beta_{1, \ell}\right\}_{\ell=1}^{\eta-1},\left\{\beta_{2, \ell}\right\}_{\ell=1}^{\eta-1},\left\{\boldsymbol{\gamma}_{1, \ell}\right\}_{\ell=1}^{\eta-1},\left\{\boldsymbol{\gamma}_{2, \ell}\right\}_{\ell=1}^{\eta-1}$ are defined as in the previous Appendix when the channel inputs $X_{1,1}$ and $X_{2,1}$ rather than being defined by (160) and (161) are now defined by (179) and (180), and where $r$ is defined by (177).

The described encodings correspond to the following parameters in the concatenated scheme:

$$
\begin{aligned}
& \tilde{\mathbf{a}}_{1} \triangleq\left(\begin{array}{llll}
\sqrt{r P_{1}} & \sqrt{\frac{P_{1}}{\beta_{1,1}}} & \cdots & \sqrt{\frac{P_{1}}{\beta_{1, \eta-1}}}
\end{array}\right)^{\top}, \\
& \tilde{\mathbf{a}}_{2} \triangleq\left(\begin{array}{llll}
\sqrt{r P_{2}} & -\sqrt{\frac{P_{2}}{\beta_{2,1}}} & \cdots & (-1)^{\eta-1} \sqrt{\frac{P_{2}}{\beta_{2, \eta-1}}}
\end{array}\right)^{\top},
\end{aligned}
$$

and

$$
\begin{aligned}
& \tilde{\mathrm{B}}_{1} \triangleq\left(\begin{array}{llll}
\mathbf{0} & -\sqrt{\frac{P_{1}}{\beta_{1,1}}} \gamma_{1,1}^{(0)} & \ldots & -\sqrt{\frac{P_{1}}{\beta_{1, \eta-1}}} \gamma_{1, \eta-1}^{(0)}
\end{array}\right)^{\top}, \\
& \tilde{\mathrm{B}}_{2} \triangleq\left(\begin{array}{llll}
\mathbf{0} & \sqrt{\frac{P_{2}}{\beta_{2,1}}} \gamma_{2,1}^{(0)} & \ldots & (-1)^{\eta} \sqrt{\frac{P_{2}}{\beta_{2, \eta-1}}} \gamma_{2, \eta-1}^{(0)}
\end{array}\right)^{\top}
\end{aligned}
$$

where $\mathbf{0}$ denotes the all-zero column-vector and where $\left\{\boldsymbol{\gamma}_{1, \ell}^{(0)}\right\}_{\ell=1}^{\eta-1}$ and $\left\{\boldsymbol{\gamma}_{2, \ell}^{(0)}\right\}_{\ell=1}^{\eta-1}$ are defined as in the previous Appendix.

The matrix $\tilde{C}$ is chosen as the LMMSE-estimation matrix $\mathrm{C}_{\text {LMMSE}}$, where recall that

$$
\mathrm{C}_{\mathrm{LMMSE}}=\tilde{\mathrm{A}}_{\mathrm{r}}^{\top}\left(\tilde{\mathrm{A}}_{\mathrm{r}} \tilde{\mathrm{A}}_{\mathrm{r}}^{\top}+N \mathrm{I}_{\eta}+\tilde{\mathrm{B}}_{\mathrm{r}}\left(\mathrm{K}_{W_{1} W_{2}} \otimes \mathrm{I}_{\eta}\right) \tilde{\mathrm{B}}_{\mathrm{r}}^{\top}\right)^{-1}
$$

where $\tilde{\mathrm{A}}_{\mathrm{r}} \triangleq\left(\begin{array}{ll}\tilde{\mathbf{a}}_{1} \quad \tilde{\mathbf{a}}_{2}\end{array}\right)$ and $\tilde{\mathrm{B}}_{\mathrm{r}} \triangleq\left(\begin{array}{cc}\tilde{\mathrm{B}}_{1} & \tilde{\mathrm{B}}_{2}\end{array}\right)$.

\section{B. Achievable Region}

Definition F.1: For each $\eta \in \mathbb{N}$ define the rate region $\tilde{\mathcal{R}}_{\eta}\left(P_{1}, P_{2}, N, \mathrm{~K}_{W_{1} W_{2}}\right)$ as the set of all rate-pairs $\left(R_{1}, R_{2}\right)$ satisfying (181a) and (181b), shown at the bottom of the page, where now (unlike in the previous Appendix)

$$
\begin{aligned}
\alpha_{1,1} & =r P_{1} \frac{r P_{2}+N}{r P_{1}+r P_{2}+N}, \\
\alpha_{2,1} & =r P_{2} \frac{r P_{1}+N}{r P_{1}+r P_{2}+N}, \\
\rho_{1} & =-\rho^{*}\left(P_{1}, P_{2}, N\right)
\end{aligned}
$$

where $r$ is the unique solution in $[0,1]$ to

$$
\sqrt{\frac{r^{2} P_{1} P_{2}}{\left(r P_{1}+N\right)\left(r P_{2}+N\right)}}=\rho^{*}\left(P_{1}, P_{2}, N\right)
$$

$$
\begin{aligned}
R_{1} \leq & \frac{1}{2 \eta} \log \left(1+\frac{r P_{1}}{N}\right)+\frac{1}{2 \eta} \sum_{\ell=2}^{\eta} \log \left(1+\frac{P_{1} \bar{\kappa}_{1, \ell-1}^{2} \frac{\alpha_{1, \ell-1}}{\beta_{1, \ell-1}}\left(1-\rho_{\ell-1}^{2}\right)}{\operatorname{Var}\left(W_{\perp, \ell-1}\right)+N}\right) \\
R_{2} \leq & \frac{1}{2 \eta} \log \left(1+\frac{r P_{2}}{N}\right)+\frac{1}{2 \eta} \sum_{\ell=2}^{\eta} \log \left(1+\frac{P_{2} \bar{\kappa}_{2, \ell-1}^{2} \frac{\alpha_{2, \ell-1}}{\beta_{2, \ell-1}}\left(1-\rho_{\ell-1}^{2}\right)}{\operatorname{Var}\left(W_{\perp, \ell-1}\right)+N}\right) \\
R_{1}+R_{2} \leq & \frac{1}{2 \eta} \log \left(1+\frac{r P_{1}+r P_{2}}{N}\right) \\
& +\frac{1}{2 \eta} \sum_{\ell=2}^{\eta} \log \left(1+\frac{P_{1} \bar{\kappa}_{1, \ell-1}^{2} \frac{\alpha_{1, \ell-1}}{\beta_{1, \ell-1}}+P_{2} \bar{\kappa}_{2, \ell-1}^{2} \frac{\alpha_{2, \ell-1}}{\beta_{2, \ell-1}}+2 \sqrt{P_{1} P_{2}} \bar{\kappa}_{1, \ell-1} \bar{\kappa}_{2, \ell-1} \sqrt{\frac{\alpha_{1, \ell-1}}{\beta_{1, \ell-1}} \frac{\alpha_{2, \ell-1}}{\beta_{2, \ell-1}}} \rho_{\ell-1}}{\operatorname{Var}\left(W_{\perp, \ell-1}\right)+N}\right)
\end{aligned}
$$


and where the parameters $\left\{\alpha_{1, \ell}\right\}_{\ell=2}^{\eta-1},\left\{\alpha_{2, \ell}\right\}_{\ell=2}^{\eta-1},\left\{\rho_{\ell}\right\}_{\ell=2}^{\eta-1}$, $\left\{\beta_{1, \ell}\right\}_{\ell=1}^{\eta-1},\left\{\beta_{2, \ell}\right\}_{\ell=1}^{\eta-1},\left\{\bar{\kappa}_{1, \ell}\right\}_{\ell=1}^{\eta-1},\left\{\bar{\kappa}_{2, \ell}\right\}_{\ell=1}^{\eta-1},\left\{W_{\perp, \ell}\right\}_{\ell=1}^{\eta-1}$ are defined as in the previous Appendix, if the input symbols $X_{1,1}$ and $X_{2,1}$ rather than being defined by (160) and (161) are now defined by (179) and (180).

Corollary F.2: For the two-user AWGN MAC with noisy feedback our concatenated scheme with the parameters described in Section F-A achieves all rate pairs in the regions $\tilde{\mathcal{R}}_{\eta}\left(P_{1}, P_{2}, N, \mathrm{~K}_{W_{1} W_{2}}\right)$ for positive integers $\eta$, i.e.

$\mathrm{C}_{\text {NoisyFB }}\left(P_{1}, P_{2}, N, \mathrm{~K}_{W_{1} W_{2}}\right)$

$$
\supseteq \mathrm{cl}\left(\bigcup_{\eta \in \mathbb{N}} \tilde{\mathcal{R}}_{\eta}\left(P_{1}, P_{2}, N, \mathrm{~K}_{W_{1} W_{2}}\right)\right) \text {. }
$$

Remark F.3: Specializing the region in Definition F.1 to perfect feedback, i.e., to $\mathrm{K}_{W_{1} W_{2}}=0$, results in the region $\tilde{\mathcal{R}}_{\eta}\left(P_{1}, P_{2}, N, 0\right)$, which is defined as the set of all rate pairs $\left(R_{1}, R_{2}\right)$ satisfying

$$
\begin{aligned}
& R_{1} \leq \frac{1}{2 \eta} \log \left(1+\frac{r P_{1}}{N}\right) \\
&+\sum_{\ell=2}^{\eta} \frac{1}{2 \eta} \log \left(1+\frac{P_{1}\left(1-\rho_{\ell-1}^{2}\right)}{N}\right), \\
& R_{2} \leq \frac{1}{2 \eta} \log \left(1+\frac{r P_{2}}{N}\right) \\
&+\sum_{\ell=2}^{\eta} \frac{1}{2 \eta} \log \left(1+\frac{P_{2}\left(1-\rho_{\ell-1}^{2}\right)}{N}\right), \\
& R_{1}+R_{2} \leq \frac{1}{2 \eta} \log \left(1+\frac{r P_{1}+r P_{2}}{N}\right) \\
&+\sum_{\ell=2}^{\eta} \frac{1}{2 \eta} \log \left(1+\frac{P_{1}+P_{2}+2}{N}\right. \\
&\left.+\frac{\sqrt{P_{1} P_{2}}(-1)^{\ell-1} \rho_{\ell-1}}{N}\right)
\end{aligned}
$$

(186c)

where the sequence $\left\{\rho_{\ell}\right\}_{\ell=1}^{\eta-1}$ is recursively defined by $\rho_{1}=$ $-\rho^{*}\left(P_{1}, P_{2}, N\right)$ and for $\ell \in\{2, \ldots, \eta\}$ by

$$
\rho_{\ell}=\frac{\rho_{\ell-1} N-(-1)^{\ell-1} \sqrt{P_{1} P_{2}}\left(1-\rho_{\ell-1}^{2}\right)}{\sqrt{P_{1}\left(1-\rho_{\ell-1}^{2}\right)+N} \sqrt{P_{2}\left(1-\rho_{\ell-1}^{2}\right)+N}}
$$

and where $r$ is the unique solution in $[0,1]$ to $(185)$.

Proof: Notice that if $\mathrm{K}_{W_{1} W_{2}}=0$, then trivially $W_{\perp, \ell}=0$, for $\ell \in\{1, \ldots, \eta-1\}$, and (165)-(168), (175), and (176) result in

$$
\begin{aligned}
& \boldsymbol{\gamma}_{\nu, \ell}=\mathrm{K}_{Y^{\ell}}^{-1} \mathrm{~K}_{Y^{\ell}, \Xi_{\nu}}, \\
& \beta_{\nu, \ell}=\operatorname{Var}\left(\Xi_{\nu}-\mathrm{K}_{Y^{\ell}, \Xi_{\nu}}^{\top} \mathrm{K}_{Y^{\ell}}^{-1} Y^{\ell}\right)=\alpha_{\nu, \ell} \\
& \bar{\kappa}_{\nu, \ell}=1 .
\end{aligned}
$$

Thus, for perfect feedback the parameters suggested in Section F-A are LMMSE-estimation error parameters, which are optimal for perfect feedback in the sense discussed in Section IV-C2. The rate expressions in (171) then result in Expressions (186), and Recursion (174) results in (187).

This concludes the proof of the remark.

\section{APPENDIX G \\ CHOICE OF PARAMETERS III}

In this section, we consider the noisy-feedback setup with receiver side-information, and we present for each $\eta \in \mathbb{N}$ a specific choice of the parameters $\mathbf{a}_{1}, \mathbf{a}_{2}, \mathrm{~B}_{1}, \mathrm{~B}_{2}, \mathrm{C}_{\mathrm{SI}}$, which we call $\breve{\mathrm{a}}_{1}, \breve{\mathrm{a}}_{2}, \breve{\mathrm{B}}_{1}, \breve{\mathrm{B}}_{2}, \breve{\mathrm{C}}_{\mathrm{SI}}$. As we shall see, the matrix $\breve{C}_{\mathrm{SI}}$ is chosen as the LMMSE-estimation matrix. Thus, the achievable region of our concatenated scheme with parameters $\eta, \breve{\mathbf{a}}_{1}, \breve{\mathrm{a}}_{2}, \breve{\mathrm{B}}_{1}, \breve{\mathrm{B}}_{2}, \breve{\mathrm{C}}_{\mathrm{SI}}$ is obtained by substituting the parameters $\breve{a}_{1}, \breve{a}_{2}, \breve{B}_{1}, \mathrm{~B}_{2}$ into (156). The resulting achievable region is presented in Corollary G.2.

\section{A. Description of Parameters}

Let a positive integer $\eta \in \mathbb{N}$ be given. We first describe how Inner Encoder 1 and Inner Encoder 2 map the fed symbols to the channel inputs. This then determines $\breve{a}_{1}, \breve{a}_{2}, \breve{B}_{1}, \breve{B}_{2}, \breve{C}$. To simplify the description we replace the symbols $\xi_{1}$ and $\xi_{2}$ fed to the inner encoders by the independent standard Gaussians $\Xi_{1}$ and $\Xi_{2}$. We choose the inner encoders to produce

$$
\begin{aligned}
& X_{1,1}=\sqrt{P_{1}} \Xi_{1}, \\
& X_{2,1}=\sqrt{P_{2}} \Xi_{2}
\end{aligned}
$$

and for $\ell \in\{2, \ldots, \eta\}$ :

$$
\begin{aligned}
X_{1, \ell} & =\sqrt{\frac{P_{1}}{\breve{\beta}_{1, \ell-1}}}\left(\Xi_{1}-\breve{\gamma}_{1, \ell-1}^{\top} V_{1}^{\ell-1}\right), \\
X_{2, \ell} & =(-1)^{\ell-1} \sqrt{\frac{P_{2}}{\breve{\beta}_{2, \ell-1}}}\left(\Xi_{2}-\breve{\gamma}_{2, \ell-1}^{\top} \mathrm{M}_{\ell-1} V_{2}^{\ell-1}\right)
\end{aligned}
$$

where for $\ell \in\{1, \ldots, \eta-1\}$ the matrix $\mathrm{M}_{\ell}$ is defined as in (164) and

$$
\begin{aligned}
& \breve{\beta}_{1, \ell} \triangleq \operatorname{Var}\left(\Xi_{1}-\breve{\gamma}_{1, \ell}^{\top} V_{1}^{\ell}\right), \\
& \breve{\beta}_{2, \ell} \triangleq \operatorname{Var}\left(\Xi_{2}-\breve{\gamma}_{2, \ell}^{\top} \mathrm{M}_{\ell} V_{2}^{\ell}\right), \\
& \breve{\gamma}_{1, \ell}=\mathrm{K}_{V_{1}^{\ell}}^{-1} \mathrm{~K}_{V_{1}^{\ell}, \Xi_{1}}, \\
& \breve{\gamma}_{2, \ell}=\mathrm{K}_{V_{2}^{\ell}}^{-1} \mathrm{~K}_{V_{2}^{\ell}, \Xi_{2}} .
\end{aligned}
$$

Notice that this choice implies that the $\ell$ th channel input produced by Inner Encoder 1 is a scaled version of the LMMSE-estimation error of $\Xi_{1}$ based on the past feedback outputs $V_{1,1}, \ldots, V_{1, \ell-1}$. Similarly, for Inner Encoder 2.

The described encodings correspond to the following parameters of the concatenated scheme:

$$
\begin{aligned}
& \breve{\mathbf{a}}_{1} \triangleq\left(\begin{array}{llll}
\sqrt{P_{1}} & \sqrt{\frac{P_{1}}{\beta_{1,1}}} & \cdots & \sqrt{{\breve{\beta_{1}}}_{1, \eta-1}}
\end{array}\right)^{\top}, \\
& \breve{\mathbf{a}}_{2} \triangleq\left(\begin{array}{llll}
\sqrt{P_{2}} & -\sqrt{\frac{P_{2}}{\bar{\beta}_{2,1}}} & \cdots & (-1)^{\eta-1} \sqrt{\frac{P_{2}}{\beta_{2, \eta-1}}}
\end{array}\right)^{\top},
\end{aligned}
$$


and

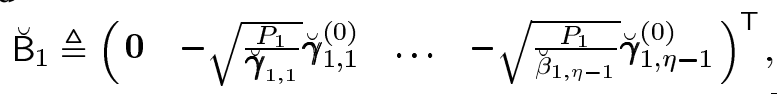

$$
\begin{aligned}
& \breve{\mathrm{B}}_{2} \triangleq\left(\begin{array}{llll}
0 & \sqrt{\frac{P_{2}}{\breve{\beta}_{2,1}}} \breve{\gamma}_{2,1}^{(0)} \quad \ldots \quad(-1)^{\eta} \sqrt{\frac{P_{2}}{\breve{\beta}_{2, \eta-1}}} \breve{\gamma}_{2, \eta-1}^{(0)}
\end{array}\right)^{\top}
\end{aligned}
$$

where the vectors $\left\{\breve{\boldsymbol{\gamma}}_{1, \ell}^{(0)}\right\}_{\ell=1}^{\eta-1}$ and $\left\{\breve{\boldsymbol{\gamma}}_{2, \ell}^{(0)}\right\}_{\ell=1}^{\eta-1}$ are defined as the $\eta$-dimensional vector obtained by stacking the $\ell$-dimensional column-vector $\breve{\gamma}_{\nu, \ell}$ on top of an $(\eta-\ell)$-dimensional columnvector with all zero entries, i.e.,

$$
\breve{\boldsymbol{\gamma}}_{\nu, \ell}^{(0)} \triangleq\left(\begin{array}{c}
\breve{\boldsymbol{\gamma}}_{\nu, \ell} \\
\mathbf{0}
\end{array}\right), \quad \ell \in\{1, \ldots, \eta-1\}, \quad \nu \in\{1,2\} .
$$

The matrix $\breve{C}_{\mathrm{SI}}$ is chosen as the LMMSE-estimation matrix with side-information, i.e.,

$$
\breve{C}_{\mathrm{SI}}=\breve{\mathrm{A}}_{\mathrm{r}}^{\top}\left(\breve{\mathrm{A}}_{\mathrm{r}} \breve{\mathrm{A}}_{\mathrm{r}}^{\top}+N \mathrm{I}_{\eta}\right)^{-1},
$$

where $\breve{A}_{\mathrm{r}} \triangleq\left(\begin{array}{ll}\breve{\mathbf{a}}_{1} & \breve{\mathbf{a}}_{2}\end{array}\right)$.

\section{B. Achievable Region}

Definition G.1: For each $\eta \in \mathbb{N}$ define the region $\breve{\mathcal{R}}_{\eta}\left(P_{1}, P_{2}, N, \mathrm{~K}_{W_{1} W_{2}}\right)$ as the set of all rate pairs $\left(R_{1}, R_{2}\right)$ satisfying

$$
\begin{aligned}
R_{1} \leq & \frac{1}{2 \eta} \sum_{\ell=1}^{\eta} \log \left(1+\frac{P_{1} \frac{\alpha_{1, \ell-1}}{\breve{\beta}_{1, \ell-1}}\left(1-\rho_{\ell-1}^{2}\right)}{N}\right), \\
R_{2} \leq & \frac{1}{2 \eta} \sum_{\ell=1}^{\eta} \log \left(1+\frac{P_{2} \frac{\alpha_{2, \ell-1}}{\breve{\beta}_{2, \ell-1}}\left(1-\rho_{\ell-1}^{2}\right)}{N}\right), \\
R_{1}+ & R_{2} \\
\leq & \frac{1}{2 \eta} \sum_{\ell=1}^{\eta} \log \left(1+\frac{P_{1} \frac{\alpha_{1, \ell-1}}{\breve{\beta}_{1, \ell-1}}+P_{2} \frac{\alpha_{2, \ell-1}}{\breve{\beta}_{2, \ell-1}}}{N}\right. \\
& \left.+\frac{2 \sqrt{P_{1} P_{2}} \sqrt{\frac{\alpha_{1, \ell-1} \alpha_{2, \ell-1}}{\breve{\beta}_{1, \ell-1} \breve{\beta}_{2, \ell-1}}}\left(1+(-1)^{\ell-1} \rho_{\ell-1}\right)}{N}\right)
\end{aligned}
$$

where $\alpha_{1,0}=1, \alpha_{2,0}=1, \rho_{0}=0$, and $\left\{\alpha_{1, \ell}\right\}_{\ell=1}^{\eta},\left\{\alpha_{2, \ell}\right\}_{\ell=1}^{\eta}$, and $\left\{\rho_{\ell}\right\}_{\ell=1}^{\eta}$ are recursively given by (199)-(201), shown at the bottom of the page, and where $\breve{\beta}_{1,0}=1, \breve{\beta}_{2,0}=1$, and $\left\{\breve{\beta}_{1, \ell}\right\}_{\ell=1}^{\eta-1}$ and $\left\{\breve{\beta}_{2, \ell}\right\}_{\ell=1}^{\eta-1}$ are described by (191)-(198).
Corollary G.2: The capacity region $\mathrm{C}_{\text {NoisyFBSI }}\left(P_{1}, P_{2}, N, \mathrm{~K}_{W_{1} W_{2}}\right)$ of the two-user Gaussian MAC with noisy feedback and receiver side-information contains the rate regions $\breve{\mathcal{R}}_{\eta}\left(P_{1}, P_{2}, N, \mathrm{~K}_{W_{1} W_{2}}\right)$ for positive integers $\eta$, i.e.,

$$
\begin{aligned}
\mathrm{C}_{\text {NoisyFBSI }}\left(P_{1}, P_{2}, N,\right. & \left.\mathrm{K}_{W_{1} W_{2}}\right) \\
& \supseteq \operatorname{cl}\left(\bigcup_{\eta \in \mathbb{N}} \breve{\mathcal{R}}_{\eta}\left(P_{1}, P_{2}, N, \mathrm{~K}_{W_{1} W_{2}}\right)\right) .
\end{aligned}
$$

\section{APPENDIX H}

\section{Rate-SPlitTing With CARLEIAL's Cover-Leung Scheme}

In this section we describe the rate-splitting scheme in Section IV-D2 in more detail. We consider the version of the scheme where after each Block $b \in\{1, \ldots, B\}$ Transmitter 1 first decodes Message $M_{2, \mathrm{CS}, b}$ before decoding $M_{2, \mathrm{CL}, b}$. Similarly, for Transmitter 2 .

We first describe the encodings. We start with the encodings in Block $b$, for a fixed $b \in\{1, \ldots, B\}$, where we assume that from decoding steps in the previous block $(b-1)$ both transmitters are cognizant of the pair $\left(M_{1, \mathrm{CL}, b-1}, M_{2, \mathrm{CL}, b-1}\right)$. Given $M_{\nu, \mathrm{CL}, b}=m_{\nu, \mathrm{CL}, b}$, $M_{1, \mathrm{CL}, b-1}=m_{1, \mathrm{CL}, b-1}$, and $M_{2, \mathrm{CL}, b-1}=m_{2, \mathrm{CL}, b-1}$, Transmitter $\nu$, for $\nu \in\{1,2\}$, picks the codewords $\mathbf{u}_{\nu, b}\left(m_{\nu, \mathrm{CL}, b}\right) \triangleq$ $\left(u_{\nu, b, 1}, \ldots, u_{\nu, b, \eta n}\right), \boldsymbol{\omega}_{1, b}\left(m_{1, \mathrm{CL}, b-1}\right) \triangleq\left(\omega_{1, b, 1}, \ldots, \omega_{1, b, \eta n}\right)$, and $\boldsymbol{\omega}_{2, b}\left(m_{2, \mathrm{CL}, b-1}\right) \triangleq\left(\omega_{2, b, 1}, \ldots, \omega_{2, b, \eta n}\right)$ from the corresponding codebooks, which have independently been generated by randomly drawing each entry according to an IID zero-mean unit-variance Gaussian distribution ${ }^{14}$. Fix correlation coefficients $\rho_{1}, \rho_{2} \in[0,1]$, which are constant over all blocks $b \in\{1, \ldots B\}$. Transmitter $\nu$ computes the following linear combinations for $k \in\{1, \ldots, n\}$ and $\nu \in\{1,2\}$ :

$$
\sqrt{\left(1-\rho_{\nu}^{2}\right) P_{\nu}^{\prime}} \mathbf{u}_{\nu, b, k}+\sqrt{\frac{1}{2} \rho_{\nu}^{2} P_{\nu}^{\prime}}\left(\boldsymbol{\omega}_{1, b, k}+\boldsymbol{\omega}_{2, b, k}\right)
$$

where

$$
\begin{aligned}
& \mathbf{u}_{\nu, b, k} \triangleq\left(u_{\nu, b,(k-1) \eta+1}, \ldots, u_{\nu, b, k \eta}\right)^{\top}, \\
& \boldsymbol{\omega}_{\nu, b, k} \triangleq\left(\omega_{\nu, b,(k-1) \eta+1}, \ldots, \omega_{\nu, b, k \eta}\right)^{\top} .
\end{aligned}
$$

${ }^{14}$ To satisfy the power constraints the Gaussian distribution should be of variance slightly less than 1 . However, this is a technicality which we ignore.

$$
\begin{aligned}
& \alpha_{1, \ell}=\alpha_{1, \ell-1}\left(1+\frac{P_{1} \frac{\alpha_{1, \ell-1}}{\beta_{1, \ell-1}}+2 \sqrt{P_{1} P_{2}}\left(1+(-1)^{\ell-1} \rho_{\ell-1}\right) \sqrt{\frac{\alpha_{1, \ell-1} \alpha_{2, \ell-1}}{\bar{\beta}_{1, \ell-1} \beta_{2, \ell-1}}}}{\frac{\alpha_{2, \ell-1}}{\widetilde{\beta}_{2, \ell-1}} P_{2}\left(1-\rho_{\ell-1}^{2}\right)+N}\right)^{-1} \\
& \alpha_{2, \ell}=\alpha_{2, \ell-1}\left(1+\frac{P_{2} \frac{\alpha_{1, \ell-1}}{\beta_{2, \ell-1}}+2 \sqrt{P_{1} P_{2}}\left(1+(-1)^{\ell-1} \rho_{\ell-1}\right) \sqrt{\frac{\alpha_{1, \ell-1} \alpha_{2, \ell-1}}{\ddot{\beta}_{1, \ell-1} \breve{\beta}_{2, \ell-1}}}}{\alpha_{1, \ell-1} P_{1}\left(1-\rho_{\ell-1}^{2}\right)+N}\right)^{-1} \\
& \rho_{\ell}=\frac{-\sqrt{P_{1} P_{2}} \sqrt{\frac{\alpha_{1, \ell-1} \alpha_{2, \ell-1}}{\widetilde{\beta}_{1, \ell-1} \ddot{\beta}_{2, \ell-1}}}\left(1-\rho_{\ell-1}^{2}\right)+\rho_{\ell-1} N}{\sqrt{P_{1} \frac{\alpha_{1, \ell-1}}{\widetilde{\beta}_{1, \ell-1}}\left(1-\rho_{\ell-1}^{2}\right)+N} \sqrt{P_{2} \frac{\alpha_{2, \ell-1}}{\widetilde{\beta}_{2, \ell-1}}\left(1-\rho_{\ell-1}^{2}\right)+N}}
\end{aligned}
$$


Moreover, Transmitter $\nu$ uses our concatenated code to encode Message $M_{\nu, \mathrm{CS}, b}$. Specifically, given $M_{\nu, \mathrm{CS}, b}=m_{\nu, \mathrm{CS}, b}$, Transmitter $\nu$ feeds $m_{\nu, \mathrm{CS}, b}$ to Outer Encoder $\nu$, which picks the codeword $\boldsymbol{\xi}_{\nu}\left(m_{\nu, \mathrm{CS}, b}\right) \triangleq\left(\xi_{\nu, b, 1}, \ldots, \xi_{\nu, b, n}\right)^{\top}$ corresponding to $m_{\nu, \mathrm{CS}, b}$ and feeds it to Inner Encoder $\nu$. Denoting the parameters of Inner Encoder $\nu$ by $\mathbf{a}_{\nu}$ and $\mathrm{B}_{\nu}$, Inner Encoder $\nu$ produces the $\eta$-dimensional vectors

$$
\mathbf{a}_{\nu} \xi_{\nu, b, k}+\mathrm{B}_{\nu} \mathbf{V}_{\nu, b, k}, \quad k \in\{k, \ldots, n\}
$$

where

$$
\mathbf{V}_{\nu, b, k} \triangleq\left(V_{\nu,(b-1) \eta n+(k-1) \eta+1}, \ldots, V_{\nu,(b-1) \eta n+k \eta}\right)^{\top} .
$$

The signal transmitted by Transmitter $\nu$ is then described by the sum of the vectors in (202) and (203) as follows. For $k \in$ $\{1, \ldots, n\}$ and $\nu \in\{1,2\}$

$$
\begin{aligned}
\mathbf{X}_{\nu, b, k} & =\sqrt{\left(1-\rho_{\nu}^{2}\right) P_{\nu}^{\prime}} \mathbf{u}_{\nu, b, k} \\
& +\sqrt{\frac{1}{2} \rho_{\nu}^{2} P_{\nu}^{\prime}}\left(\boldsymbol{\omega}_{1, b, k}+\boldsymbol{\omega}_{2, b, k}\right)+\mathbf{a}_{\nu} \xi_{\nu, b, k}+\mathrm{B}_{\nu} \mathbf{V}_{\nu, b, k}
\end{aligned}
$$

where

$$
\mathbf{X}_{\nu, b, k} \triangleq\left(X_{\nu,(b-1) \eta n+(k-1) \eta+1}, \ldots, X_{\nu,(b-1) \eta n+k \eta}\right)^{\top} .
$$

Notice that if $\mathbf{a}_{1}, \mathbf{a}_{2}, \mathrm{~B}_{1}$, and $\mathrm{B}_{2}$ satisfy the power constraints (19) for powers $\left(P_{1}-P_{1}^{\prime}\right)$ and $\left(P_{2}-P_{2}^{\prime}\right)$, noise variance $(N+$ $\left.P_{1}^{\prime}+P_{2}^{\prime}+2 \sqrt{P_{1}^{\prime} P_{2}^{\prime}} \rho_{1} \rho_{2}\right)$, and feedback-noise covariance matrix $\mathrm{K}_{W_{1} W_{2}}$ and if the outer code's codewords $\left\{\boldsymbol{\Xi}_{1}\left(M_{1, \mathrm{CS}, \boldsymbol{b}}\right)\right\}$ and $\left\{\boldsymbol{\Xi}_{2}\left(M_{2, \mathrm{CS}, b}\right)\right\}$ are zero-mean and average block-power constrained to 1 , then the channel input sequences satisfy the power constraints with arbitrary high probability.

In Block $(B+1)$, the two transmitters only send information about the pair $\left(M_{1, \mathrm{CL}, B}, M_{2, \mathrm{CL}, B}\right)$. Given $M_{1, \mathrm{CL}, B}=$ $m_{1, \mathrm{CL}, B}$ and $M_{2, \mathrm{CL}, B}=m_{2, \mathrm{CL}, B}$, both transmitters pick the codewords $\boldsymbol{\omega}_{1, B+1}\left(m_{1, \mathrm{CL}, \mathrm{B}}\right) \triangleq\left(\omega_{1, B+1,1}, \ldots, \omega_{1, B+1, \eta n}\right)^{\mathrm{\top}}$ and $\boldsymbol{\omega}_{2, B+1}\left(m_{2, \mathrm{CL}, \mathrm{B}}\right) \triangleq\left(\omega_{2, B+1,1}, \ldots, \omega_{2, B+1, \eta n}\right)^{\top}$ from the corresponding codebooks and form a linear combination of power $P_{\nu}^{\prime}$. Thus, defining

$$
\begin{aligned}
& \mathbf{X}_{\nu, B+1} \triangleq\left(X_{\nu, B \eta n+1}, \ldots, X_{\nu,(B+1) \eta n}\right)^{\top}, \\
& \boldsymbol{\omega}_{\nu, B+1} \triangleq\left(\omega_{\nu, B+1,1}, \ldots, \omega_{\nu, B+1, \eta n}\right)^{\top}
\end{aligned}
$$

the signal transmitted by Transmitter $\nu$ can be described as

$$
\mathbf{X}_{\nu, B+1}=\sqrt{\frac{1}{2} \rho_{\nu}^{2} P_{\nu}^{\prime}}\left(\boldsymbol{\omega}_{1, B+1}+\boldsymbol{\omega}_{2, B+1}\right)
$$

Next, we describe the decodings. We start with the decoding at Transmitter 2; the decoding at Transmitter 1 is performed similarly and therefore omitted; and the decodings at the receiver are described later on.

Recall that after a fixed block $b$, for $b \in\{1, \ldots, B\}$, Transmitter 2 first decodes Message $M_{1, \mathrm{CS}, b}$, followed by Message $M_{1, \mathrm{CL}, b}$. After Block $b$, Transmitter 2 observed $\left\{\mathbf{V}_{2, b, 1}, \ldots, \mathbf{V}_{2, b, n}\right\}$, and additionally is cognizant of the realizations of $\left\{\mathbf{U}_{2, b, 1}, \ldots, \mathbf{U}_{2, b, n}\right\}, \quad\left\{\boldsymbol{\Omega}_{1, b, 1}, \ldots, \boldsymbol{\Omega}_{1, b, n}\right\}$,
$\left\{\boldsymbol{\Omega}_{2, b, 1}, \ldots, \boldsymbol{\Omega}_{2, b, n}\right\}$, and $\left\{\Xi_{2, b, 1}, \ldots, \Xi_{2, b, n}\right\}$. It can thus compute for $k \in\{1, \ldots, n\}$ :

$$
\begin{aligned}
\tilde{\mathbf{V}}_{2, b, k} \triangleq & \left(\mathrm{I}-\left(\mathrm{B}_{1}+\mathrm{B}_{2}\right)\right) \mathbf{V}_{2, b, k} \\
& -\sqrt{\left(1-\rho_{2}^{2}\right) P_{2}^{\prime}} \mathbf{U}_{2, b, k} \\
& -\left(\sqrt{\frac{1}{2} \rho_{1}^{2} P_{1}^{\prime}}+\sqrt{\frac{1}{2} \rho_{2}^{2} P_{2}^{\prime}}\right)\left(\mathbf{\Omega}_{1, b, k}+\boldsymbol{\Omega}_{2, b, k}\right) \\
& -\mathbf{a}_{2} \Xi_{2, b, k} \\
= & \mathbf{a}_{1} \cdot \Xi_{1, b, k}+\sqrt{\left(1-\rho_{1}^{2}\right) P_{1}^{\prime}} \mathbf{U}_{1, b, k}+\mathbf{Z}_{b, k} \\
& +\mathbf{W}_{2, b, k}+\mathrm{B}_{1}\left(\mathbf{W}_{1, b, k}-\mathbf{W}_{2, b, k}\right),
\end{aligned}
$$

where

$$
\begin{aligned}
\mathbf{Z}_{b, k} & \triangleq\left(Z_{(b-1) \eta n+(k-1) n+1}, \ldots, Z_{(b-1) \eta n+k n}\right)^{\top}, \\
\mathbf{W}_{\nu, b, k} & \triangleq\left(W_{\nu,(b-1) \eta n+(k-1) n+1}, \ldots, W_{\nu,(b-1) \eta n+k n}\right)^{\top} .
\end{aligned}
$$

Since the sequence $\left\{\tilde{\mathbf{V}}_{2, b, 1}, \ldots, \tilde{\mathbf{V}}_{2, b, n}\right\}$ is independent of the additional information $\left\{\mathbf{U}_{2, b, 1}, \ldots, \mathbf{U}_{2, b, n}\right\}$, $\left\{\boldsymbol{\Omega}_{1, b, 1}, \ldots, \boldsymbol{\Omega}_{1, b, n}\right\}, \quad\left\{\boldsymbol{\Omega}_{2, b, 1}, \ldots, \boldsymbol{\Omega}_{2, b, n}\right\}, \quad$ and $\left\{\Xi_{2, b, 1}, \ldots, \Xi_{2, b, n}\right\}$, Transmitter 2 can optimally decode Message $M_{1, \mathrm{CS}, b}$ based on $\left\{\tilde{\mathbf{V}}_{2, b, 1}, \ldots, \tilde{\mathbf{V}}_{2, b, n}\right\}$ only. To this end, it does not apply the inner and outer decoder of the concatenated scheme, but directly applies an optimal decoder for a Gaussian single-input antenna/ $\eta$-output antenna channel with temporally-white noise sequences which are correlated across antennas. Let $\hat{M}_{1, \mathrm{CS}}^{(\mathrm{T} 2)}$ denote Transmitter 2's guess of Message $M_{1, \mathrm{CS}}$ and let $\left(\hat{\Xi}_{1, b, 1}^{(\mathrm{Tx} 2)}, \ldots, \hat{\Xi}_{1, b, n}^{(\mathrm{Tx} 2)}\right)^{\top}$ be the corresponding codeword of the outer code.

Transmitter 2 then decodes Message $M_{1, \mathrm{CL}, b}$ as follows. It first attempts to subtract the influence of the sequence produced by encoding $M_{1, \mathrm{CS}, b}$ and to this end computes

$$
\tilde{\mathbf{V}}_{2, b, k}^{(2)} \triangleq \tilde{\mathbf{V}}_{2, b, k}-\mathbf{a}_{1} \hat{\Xi}_{1, b, k}^{(\operatorname{Tx} 2)}, \quad k \in\{1, \ldots, n\},
$$

which, if Transmitter 2 successfully decoded $M_{1, \mathrm{CS}, b}$, equals

$$
\begin{aligned}
& \sqrt{\left(1-\rho_{1}^{2}\right) P_{1}^{\prime}} \mathbf{U}_{1, b, k}+\mathbf{Z}_{b, k}+\mathbf{W}_{2, b, k} \\
&+\mathbf{B}_{1}\left(\mathbf{W}_{1, b, k}-\mathbf{W}_{2, b, k}\right), \quad k \in\{1, \ldots, n\} .
\end{aligned}
$$

Transmitter 2 then decodes Message $M_{1, \mathrm{CL}, b}$ based on the sequences $\left\{\tilde{\mathbf{V}}_{2, b, 1}^{(2)}, \ldots, \tilde{\mathbf{V}}_{2, b, n}^{(2)}\right\}$ using an optimal decoder for a Gaussian $\eta$-input antenna/ $\eta$-output antenna channel with temporally-white noise sequences correlated across antennas.

As a last element, we describe the decodings at the receiver. After each block $b \in\{1, \ldots, B\}$ the receiver performs two decoding steps. In the first step it decodes Messages $\left(M_{1, \mathrm{CS}, b}, M_{2, \mathrm{CS}, b}\right)$ while treating the sequences produced to encode $M_{1, \mathrm{CL}, b-1}, M_{2, \mathrm{CL}, b-2}, \quad M_{1, \mathrm{CL}, b}$, and $M_{2, \mathrm{CL}, b}$ as additional noise. For this decoding step the receiver uses inner and outer decoders of the concatenated scheme. Let $\left(\hat{M}_{1, \mathrm{CS}, b}, \hat{M}_{2, \mathrm{CS}, b}\right)$ denote the receiver's guess of the pair $\left(M_{1, \mathrm{CS}, b}, M_{2, \mathrm{CS}, b}\right)$ produced in this first step, and let $\left(\hat{\Xi}_{1, b, 1}^{(\mathrm{Rx})} \ldots, \hat{\Xi}_{1, b, n}^{(\mathrm{Rx})}\right)^{\top}$ and $\left(\hat{\Xi}_{2, b, 1}^{(\mathrm{Rx})}, \ldots, \hat{\Xi}_{2, b, n}^{(\mathrm{Rx})}\right)^{\top}$ be the corresponding codewords of the outer code. 
In the second decoding step, the receiver decodes Messages $M_{1, \mathrm{CL}, b-1}$ and $M_{2, \mathrm{CL}, b-1}$. To this end, it first pre-processes the outputs observed in blocks $b$ and $b-1$ to mitigate the influence of the sequences produced to encode Messages $\left(M_{1, \mathrm{CS}, b}, M_{2, \mathrm{CS}, b}\right)$. The outputs in block $b$ are processed as follows: For each $k \in\{1, \ldots, n\}$ the receiver computes

$\tilde{\mathbf{Y}}_{b, k} \triangleq \mathbf{Y}_{b, k}-\mathbf{a}_{1} \hat{\Xi}_{1, b, k}^{(\mathrm{Rx})}-\mathbf{a}_{2} \hat{\Xi}_{2, b, k}^{(\mathrm{Rx})}-\mathrm{B}_{1} \mathbf{Y}_{b, k}-\mathrm{B}_{2} \mathbf{Y}_{b, k}$

where

$$
\mathbf{Y}_{b, k} \triangleq\left(Y_{(b-1) \eta n+(k-1) \eta+1}, \ldots, Y_{(b-1) \eta n+k \eta}\right)^{\top} .
$$

Notice that in case the first decoding step was successful, i.e, in case that $\hat{\Xi}_{1, b, k}^{(\mathrm{Rx})}=\Xi_{1, b, k}$ and $\hat{\Xi}_{2, b, k}^{(\mathrm{Rx})}=\Xi_{2, b, k}$ holds for all $k \in\{1, \ldots, n\},(206)$ corresponds to

$$
\begin{aligned}
& \sqrt{\left(1-\rho_{1}^{2}\right) P_{1}^{\prime}} \mathbf{U}_{1, b, k}+\sqrt{\left(1-\rho_{2}^{2}\right) P_{2}^{\prime}} \mathbf{U}_{2, b, k} \\
& +\left(\sqrt{\frac{1}{2} \rho_{1}^{2} P_{1}^{\prime}}+\sqrt{\frac{1}{2} \rho_{2}^{2} P_{2}^{\prime}}\right)\left(\boldsymbol{\Omega}_{1, b, k}+\boldsymbol{\Omega}_{2, b, k}\right) \\
& +\mathrm{B}_{1} \mathbf{W}_{1, b, k}+\mathrm{B}_{2} \mathbf{W}_{2, b, k}+\mathbf{Z}_{b, k} .
\end{aligned}
$$

Before describing how the receiver processes the outputs in block $b-1$, we notice that the receiver already decoded Messages $M_{1, \mathrm{CL}, b-2}, M_{2, \mathrm{CL}, b-2}, M_{1, \mathrm{CS}, b-1}$, and $M_{2, \mathrm{CS}, b-1}$ in previous decoding steps. Let $\hat{M}_{1, \mathrm{CL}, b-2}^{(\mathrm{Rx})}, \hat{M}_{2, \mathrm{CL}, b-2}^{(\mathrm{Rx})}$, $\hat{M}_{1, \mathrm{CS}, b-1}^{(\mathrm{Rx})}$, and $\hat{M}_{2, \mathrm{CS}, b-1}^{\mathrm{Rx})}$ denote the receiver's guess of these messages. Also, for each $k \in\{1, \ldots, n\}$ let $\hat{\boldsymbol{\Omega}}_{1, b-1, k}^{(\mathrm{Rx})}$ and $\hat{\Omega}_{2, b-1, k}^{(\mathrm{Rx})}$ denote the codewords that in the codebooks used in the $k$ th subblock of block $b-1$ correspond to the guesses $\hat{M}_{1, \mathrm{CL}, b-2}^{(\mathrm{Rx})}, \quad \hat{M}_{2, \mathrm{CL}, b-2}^{(\mathrm{Rx})}, \quad$ and let $\left(\hat{\Xi}_{1, b-1,1}^{(\mathrm{Rx})} \ldots, \hat{\Xi}_{1, b-1, n}^{\mathrm{Rx})}\right)^{\top}$ and $\left(\hat{\Xi}_{2, b-1,1}^{(\mathrm{Rx})}, \ldots, \hat{\Xi}_{2, b-1, n}^{(\mathrm{Rx})}\right)^{\top}$ denote the codewords that in the outer code used in block $b-1$ correspond to the guesses $\hat{M}_{1, \mathrm{CS}, b-1}^{(\mathrm{Rx})}$, and $\hat{M}_{2, \mathrm{CS}, b-1}^{\mathrm{Rx})}$. The receiver processes the outputs observed in the block $(b-1)$ by computing for $k \in\{1, \ldots, n\}$ :

$$
\begin{aligned}
& \tilde{\mathbf{Y}}_{b-1, k}^{(2)} \\
& \triangleq \mathbf{Y}_{b-1, k} \\
& \quad-\left(\sqrt{\frac{1}{2} \rho_{1}^{2} P_{1}^{\prime}}+\sqrt{\frac{1}{2} \rho_{2}^{2} P_{2}^{\prime}}\right)\left(\hat{\Omega}_{1, b-1, k}+\hat{\Omega}_{2, b-1, k}\right) \\
& \quad-\mathbf{a}_{1} \Xi_{1, b-1, k}-\mathbf{a}_{2} \Xi_{2, b-1, k}-\mathrm{B}_{1} \mathbf{Y}_{b, k}-\mathrm{B}_{2} \mathbf{Y}_{b, k}, \\
& =\sqrt{\left(1-\rho_{1}^{2}\right) P_{1}^{\prime}} \mathbf{U}_{1, b-1, k}+\sqrt{\left(1-\rho_{2}^{2}\right) P_{2}^{\prime}} \mathbf{U}_{2, b-1, k} \\
& \quad+\mathrm{B}_{1} \mathbf{W}_{1, b-1, k}+\mathrm{B}_{2} \mathbf{W}_{2, b-1, k}+\mathbf{Z}_{b-1, k} .
\end{aligned}
$$

Equipped with the sequences $\left\{\left(\tilde{\mathbf{Y}}_{b, i}, \tilde{\mathbf{Y}}_{b-1, i}^{(2)}\right)\right\}_{i=1}^{n}$ the receiver finally decodes Messages $\left(M_{1, \mathrm{CL}, b-1}, M_{2, \mathrm{CL}, b-1}\right)$ using an optimal decoder for a $2 \eta$-input antenna/ $2 \eta$-output antenna Gaussian MAC with temporally-white noise that is correlated across antennas.

After Block $(B+1)$ the receiver decodes Messages $\left(M_{1, \mathrm{CL}, B}, M_{2, \mathrm{CL}, B}\right)$ based on $\tilde{\mathbf{Y}}_{B, 1}^{(2)}, \ldots, \tilde{\mathbf{Y}}_{B, n}^{(2)}$ and based on the sequence $\left(Y_{B \eta n+1}, \ldots, Y_{(B+1) \eta n}\right)$. To this end, it again uses an optimal decoder for a $2 \eta$-input antenna/2 $\eta$-output antenna Gaussian MAC with temporally-white noise that is correlated across antennas.

\section{A. Noisy and Perfect Partial Feedback}

The proposed extension applies also to settings with noisy or perfect partial feedback to Transmitter 2 , if $\mathrm{B}_{1}$ is set to the all-zero matrix and if Carleial's scheme for partial feedback is applied. Thus, our scheme should be modified so that there are no decodings taking place at Transmitter 1 and so that in (204) and (205) the term $\sqrt{\frac{1}{2} \rho_{\nu}^{2} P_{\nu}^{\prime}}\left(\boldsymbol{\omega}_{1, b, i}+\boldsymbol{\omega}_{2, b, i}\right)$ is replaced by $\sqrt{\rho_{\nu}^{2} P_{\nu}^{\prime}} \boldsymbol{\omega}_{1, b, i}$.

Notice that in a setting with perfect partial feedback to Transmitter 2 the components of the noise vectors corrupting $\left\{\tilde{\mathbf{V}}_{2, b, i}\right\}$ are uncorrelated, similarly for $\left\{\left(\tilde{\mathbf{V}}_{2, b, i}^{(2)}-\sqrt{\left(1-\rho_{1}^{2} P_{1}^{\prime}\right)} \mathbf{u}_{1, b-1, i}\right)\right\} \quad$ and $\quad$ for $\quad \tilde{\mathbf{Y}}_{b, i}$ and $\tilde{\mathbf{Y}}_{b, i}^{(2)}$. Thus, optimal decoders for Gaussian multi-input antenna/multi-output antenna channels with uncorrelated white noise sequences can be used to decode $M_{1, \mathrm{CL}, b}$ at Transmitter 2 and to decode $\left(M_{1, \mathrm{CL}, b}, M_{2, \mathrm{CL}, b}\right)$ at the receiver. Moreover, the observation $\left\{\mathbf{Y}_{b, i}\right\}$ at the receiver is a degraded version of the observation $\left\{\tilde{\mathbf{V}}_{1, b, i}\right\}$ at Transmitter 2 . Thus, since the receiver decodes $\left(M_{1, \mathrm{CS}, b}, M_{2, \mathrm{CS}, b}\right)$ based on $\left\{\mathbf{Y}_{b, i}\right\}$, in settings with perfect partial feedback there is no loss in optimality in the presented rate-splitting scheme if based on $\left\{\tilde{\mathbf{V}}_{1, b, i}\right\}$ Transmitter 2 first decodes message $M_{1, \mathrm{CS}, b}$ before decoding $M_{1, \mathrm{CL}, b}$. In particular, the set of achievable rates of the concatenated scheme is solely constrained by the decoding at the receiver.

\section{APPENDIX I}

\section{INTERLEAVING AND RATE-SPLITTING WITH CARLEIAL'S COVER-LEUNG SCHEME}

We describe the scheme in Section IV-D3 in more detail. We start with the encodings and first consider the encodings in the $\ell$ th subblock of Block $b$, for a fixed $b \in\{1, \ldots, B\}$ and $\ell \in\{1, \ldots, \eta\}$. Define $\tilde{b}=(b-1) \eta+\ell$. We assume that from decoding steps after previous subblocks $((b-2) \eta+1), \ldots,(\tilde{b}-1)$, both transmitters are cognizant of $\left\{\left(M_{1, \mathrm{ICL},(b-2) \eta+1}, M_{2, \mathrm{ICL},(b-2) \eta+1}\right), \ldots, \quad\left(M_{1, \mathrm{ICL}, \tilde{b}-1}\right.\right.$, $\left.\left.M_{2, \mathrm{ICL}, \tilde{b}-1}\right)\right\}$.

The encodings in Subblock $\tilde{b}$ consist of four steps. In the first step Transmitter 1 produces an $n$-length vector to encode messages $M_{1, \mathrm{ICL}, \tilde{b}}, M_{1, \mathrm{ICL}, \tilde{b}-\eta}$, and $M_{2, \mathrm{ICL}, \tilde{b}-\eta}$ as follows. Given $M_{1, \mathrm{ICL}, \tilde{b}}=m_{1, \mathrm{ICL}, \tilde{b}}, M_{1, \mathrm{ICL}, \tilde{b}-\eta}=m_{1, \mathrm{ICL}, \tilde{b}-\eta}$, and $M_{2, \mathrm{ICL}, \tilde{b}-\eta}=m_{2, \mathrm{ICL}, \tilde{b}-\eta}$, Transmitter 1 first picks codewords $\mathbf{u}_{1, \tilde{b}}\left(M_{1, \mathrm{ICL}, \tilde{b}}\right), \boldsymbol{\omega}_{1, \tilde{b}}\left(M_{1, \mathrm{ICL}, \tilde{b}-\eta}\right)$, and $\boldsymbol{\omega}_{2, \tilde{b}}\left(M_{2, \mathrm{ICL}, \tilde{b}-\eta}\right)$ from the corresponding codebooks, which have independently been generated by randomly drawing each entry according to an IID zero-mean unit-variance Gaussian distribution ${ }^{15}$. Transmitter 1 then completes the first step by computing the following linear combination

$$
\sqrt{\left(1-\rho_{1}^{2}\right) P_{1}^{\prime}} \mathbf{u}_{1, \tilde{b}}+\sqrt{\frac{1}{2} \rho_{1}^{2} P_{1}^{\prime}}\left(\boldsymbol{\omega}_{1, \tilde{b}}+\boldsymbol{\omega}_{2, \tilde{b}}\right)
$$

${ }^{15}$ To satisfy the power constraints the Gaussian distribution should be of variance slightly less than 1 . However, this is a technicality which we ignore. 
where $\rho_{1} \in[0,1]$ is a fixed chosen parameter of the scheme, which does not depend on $\tilde{b}$. Similarly, for Transmitter 2 .

In the second step, Transmitter 1 computes the "cleaned" feedback vectors $\overline{\mathbf{V}}_{\nu,(b-1) \eta+1}, \ldots, \overline{\mathbf{V}}_{\nu,(b-1) \eta+\ell-1}$, where $\overline{\mathbf{V}}_{\nu, \tilde{b}^{\prime}}$ for $\tilde{b}^{\prime} \in\{(b-1) \eta+1, \ldots,(b-1) \eta+\ell-1\}$ is defined as

$$
\begin{aligned}
\overline{\mathbf{V}}_{1, \tilde{b}^{\prime}} \triangleq & \mathbf{V}_{1, \tilde{b}^{\prime}}-\sqrt{\left(1-\rho_{1}^{2}\right) P_{1}^{\prime}} \mathbf{U}_{1, \tilde{b}^{\prime}} \\
& -\sqrt{\left(1-\rho_{2}^{2}\right) P_{2}^{\prime}} \mathbf{U}_{2, \tilde{b}^{\prime}} \\
& -\left(\sqrt{\frac{1}{2} \rho_{1}^{2} P_{1}^{\prime}}+\sqrt{\frac{1}{2} \rho_{2}^{2} P_{2}^{\prime}}\right)\left(\boldsymbol{\Omega}_{1, \tilde{b}^{\prime}}+\boldsymbol{\Omega}_{2, \tilde{b}^{\prime}}\right)
\end{aligned}
$$

where $\mathbf{V}_{\nu, \tilde{b}^{\prime}} \triangleq\left(V_{\nu,\left(\tilde{b}^{\prime}-1\right) n+1}, \ldots, V_{\nu, \tilde{b}^{\prime} n}\right)^{\top}$. Similarly, for Transmitter 2. Notice that for $\tilde{b}^{\prime} \in\{(b-1) \eta+1, \ldots,(b-$ 1) $\eta+\ell-1\}$ the "cleaned" feedback vectors satisfy

$$
\overline{\mathbf{V}}_{1, \tilde{b}^{\prime}}-\mathbf{W}_{1, \tilde{b}^{\prime}}=\overline{\mathbf{V}}_{2, \tilde{b}^{\prime}}-\mathbf{W}_{2, \tilde{b}^{\prime}}
$$

where for $\tilde{b}^{\prime} \in\{(b-1) \eta, \ldots,(b-1) \eta+\ell-1\}$ and $\nu \in\{1,2\}$ :

$$
\mathbf{W}_{\nu, \tilde{b}^{\prime}} \triangleq\left(W_{\nu,\left(\tilde{b}^{\prime}-1\right) n+1}, \ldots, W_{\nu, \tilde{b}^{\prime} n}\right)^{\top} .
$$

Thus, they correspond to the feedback vectors of a "cleaned" channel where the channel outputs are described by the vectors $\left\{\left(\overline{\mathbf{V}}_{1, \tilde{b}^{\prime}}-\mathbf{W}_{1, \tilde{b}^{\prime}}\right)\right\}$.

In the third step, Transmitter 1 produces an $n$-length vector to encode Message $M_{1, \text { ICS, } b}$ using the "cleaned" feedback vectors in (208) as explained shortly. Assume that at the beginning of Block $b$ Transmitter 1 fed Message $M_{1, \text { ICS }, b}$ to its outer encoder and that the outer encoder produced the codeword $\boldsymbol{\xi}_{1, b}$. Let

$$
\begin{aligned}
\mathbf{a}_{1} & \triangleq\left(a_{1,1}, \ldots, a_{1, \eta}\right)^{\mathrm{T}}, \\
\mathrm{B}_{1} & \triangleq\left(\begin{array}{ccc}
b_{1,1,1} & \ldots & b_{1,1, \eta} \\
\ldots & & \ldots \\
b_{1, \eta, 1} & \ldots & b_{1, \eta, \eta}
\end{array}\right)
\end{aligned}
$$

denote the parameters of Transmitter 1's modified inner encoder. The modified inner encoder then produces the $n$-length vector

$$
a_{1, \ell} \boldsymbol{\xi}_{1, b}+\sum_{j=1}^{\ell-1} b_{1, \ell, j} \overline{\mathbf{V}}_{1,(b-1) \eta+j}
$$

which is also the $n$-length vector that Transmitter 1 produces in this third step. Similarly, for Transmitter 2.

In the forth and last step, Transmitter 1 sums the $n$-length vectors in (207) and (209), and sends the resulting symbols over the channel. Similarly, for Transmitter 2.

Thus, the signal transmitted by Transmitter $\nu$ in Subblock $\tilde{b}$ can be described as follows:

$$
\begin{array}{r}
\mathbf{X}_{\nu, \tilde{b}}=\sqrt{\left(1-\rho_{\nu}^{2}\right) P_{\nu}^{\prime}} \mathbf{u}_{\nu, \tilde{b}}+\sqrt{\frac{1}{2} \rho_{\nu}^{2} P_{\nu}^{\prime}}\left(\omega_{1, \tilde{b}}+\omega_{2, \tilde{b}}\right) \\
+\mathbf{a}_{\nu, \ell} \boldsymbol{\xi}_{\nu, b}+\sum_{j=1}^{\ell-1} b_{\nu, \ell, j} \overline{\mathbf{V}}_{\nu,(b-1) \eta+j}
\end{array}
$$

where $\mathbf{X}_{\nu, \tilde{b}} \triangleq\left(X_{\nu,(\tilde{b}-1) n+1}, \ldots, X_{\nu, \tilde{b} n}\right)^{\top}$.
Notice that if the parameters $\left(\mathbf{a}_{1}, \mathbf{a}_{2}, \mathrm{~B}_{1}, \mathrm{~B}_{2}\right)$ satisfy the power constraints (19) for transmit powers $\left(P_{1}-P_{1}^{\prime}\right)$ and $\left(P_{2}-P_{2}^{\prime}\right)$, noise variance $N$, and feedback-noise covariance matrix $\mathrm{K}_{W_{1} W_{2}}$, then the input sequences satisfy the power constraints (4) with arbitrary high probability.

We next consider the encodings in the last Block $(B+1)$, where the two transmitters send information about the pairs of messages $\left\{\left(M_{1, \mathrm{CL},(B-1) \eta+1}, M_{2, \mathrm{CL},(B-1) \eta+1}\right), \ldots,\left(M_{1, \mathrm{CL}, B \eta}, M_{2, \mathrm{CL}, B \eta}\right)\right\}$. We consider a fixed subblock $\tilde{b} \in\{B \eta+1, \ldots,(B+1) \eta\}$. The transmitters send their channel inputs in this last block $(B+1)$ as follows. Given $M_{1, \mathrm{CL}, \tilde{b}-\eta}=m_{1, \mathrm{CL}, \tilde{b}-\eta}$ and $M_{2, \mathrm{CL}, \tilde{b}-\eta}=m_{2, \mathrm{CL}, \tilde{b}-\eta}$, both transmitters choose the codewords $\boldsymbol{\omega}_{1, \tilde{b}}\left(M_{1, \mathrm{CL}, \tilde{b}-\eta}\right)$, and $\boldsymbol{\omega}_{2, \tilde{b}}\left(M_{2, \mathrm{CL}, \tilde{b}-\eta}\right)$ from the corresponding codebooks and send a linear combination of the chosen codewords over the channel. Thus, the signal transmitted by Transmitter $\nu$ in Subblock $\tilde{b}$ can be described as

$$
\mathbf{X}_{\nu, \tilde{b}}=\sqrt{\frac{1}{2} \rho_{\nu}^{2} P_{\nu}^{\prime}}\left(\omega_{1, \tilde{b}}+\omega_{2, \tilde{b}}\right)
$$

where

$$
\mathbf{X}_{\nu, \tilde{b}} \triangleq\left(X_{\nu,(\tilde{b}-1) n+1}, \ldots, X_{\nu, \tilde{b} n}\right)^{\top} .
$$

We next describe the decoding at Transmitter 2; the decoding at Transmitter 1 is performed similarly and therefore omitted; and the decoding at the receiver will be described later.

After each subblock $\tilde{b} \in\{1, \ldots, B \eta\}$ Transmitter 2 decodes Message $M_{1, \mathrm{ICL}, \tilde{b}}$. We consider a fixed Subblock $\tilde{b} \in\{1, \ldots, B \eta\}$ and define $b \in\{1, \ldots, B\}$ and $\ell \in\{1, \ldots, \eta\}$ so that $\tilde{b}=(b-1) \eta+\ell$. Before describing the decoding of Message $M_{1, \mathrm{ICL}, \tilde{b}}$ at the end of this paragraph, we notice the following. After Subblock $\tilde{b}$, Transmitter $2 \mathrm{ob}-$ served the feedback vectors $\mathbf{V}_{2,(b-1) \eta+1}, \ldots, \mathbf{V}_{2,(b-1) \eta+\ell}$ and is additionally cognizant of Messages $M_{2, \mathrm{ICS}, b}$, $\left\{M_{2, \mathrm{ICL},(b-1) \eta+1}, \ldots, M_{2, \mathrm{ICL},(b-1) \eta+\ell}\right\}, \quad$ and $\quad$ (assuming its previous decoding steps were successful) of Messages $\left\{M_{1, \mathrm{ICL},(b-1) \eta+1}, \ldots, M_{1, \mathrm{ICL},(b-1) \eta+\ell-1}\right\}$. It can therefore reconstruct the sequences produced to encode these messages. Moreover, Transmitter 2 can estimate Transmitter 1 's feedback outputs $\mathbf{V}_{1,(b-1) \eta+1}, \ldots, \mathbf{V}_{1,(b-1) \eta+\ell}$, (even though it cannot reconstruct them because it is incognizant of the feedback noises). By subtracting the reconstructed sequences and the estimated sequence from its feedback outputs Transmitter 2 can thus compute the $n$-dimensional vectors $\tilde{\mathbf{N}}_{2,(b-1) \eta+1}, \ldots, \tilde{\mathbf{N}}_{2,(b-1) \eta+\ell-1}$ and $\tilde{\mathbf{V}}_{2,(b-1) \eta+\ell}$, which are defined as

$$
\begin{aligned}
\tilde{\mathbf{V}}_{2,(b-1) \eta+\ell} & \triangleq \\
\triangleq & \mathbf{V}_{2,(b-1) \eta+\ell}-\sqrt{\left(1-\rho_{2}^{2}\right) P_{2}^{\prime}} \mathbf{U}_{2,(b-1) \eta+\ell} \\
- & \left(\sqrt{\frac{1}{2} \rho_{1}^{2} P_{1}^{\prime}}+\sqrt{\frac{1}{2} \rho_{2}^{2} P_{2}^{\prime}}\right) \\
& \cdot\left(\boldsymbol{\Omega}_{1,(b-1) \eta+\ell}+\boldsymbol{\Omega}_{2,(b-1) \eta+\ell}\right) \\
- & a_{2, \ell} \boldsymbol{\Xi}_{2, b}-\sum_{j=1}^{\ell-1}\left(b_{1, \ell, j}+b_{2, \ell, j}\right) \overline{\mathbf{V}}_{2,(b-1) \eta+j}
\end{aligned}
$$




$$
\begin{aligned}
= & \sqrt{\left(1-\rho_{1}^{2}\right) P_{1}^{\prime}} \mathbf{U}_{1,(b-1) \eta+\ell}+a_{1, \ell} \boldsymbol{\Xi}_{1, b} \\
& +\sum_{j=1}^{\ell-1} b_{1, \ell, j}\left(\mathbf{W}_{1,(b-1) \eta+j}-\mathbf{W}_{2,(b-1) \eta+j}\right) \\
& +\mathbf{Z}_{(b-1) \eta+\ell}+\mathbf{W}_{2,(b-1) \eta+\ell}
\end{aligned}
$$

where we define the vector $\mathbf{Z}_{(b-1) \eta+\ell}$ $\left(Z_{((b-1) \eta+\ell-1) n+1}, \ldots, Z_{((b-1) \eta+\ell) n}\right)^{\mathrm{T}} ; \quad$ and $\tilde{b}^{\prime}=(b-1)+\ell^{\prime}$ and $\ell^{\prime} \in\{1, \ldots, \ell-1\}$ :

$$
\begin{aligned}
\tilde{\mathbf{N}}_{2, \tilde{b}^{\prime}} \triangleq & \mathbf{V}_{2, \tilde{b}^{\prime}}-\sqrt{\left(1-\rho_{1}^{2}\right) P_{1}^{\prime}} \mathbf{U}_{1, \tilde{b}^{\prime}} \\
& -\sqrt{\left(1-\rho_{2}^{2}\right) P_{2}^{\prime}} \mathbf{U}_{2, \tilde{b}^{\prime}} \\
& -\left(\sqrt{\frac{1}{2} \rho_{1}^{2} P_{1}^{\prime}}+\sqrt{\frac{1}{2} \rho_{2}^{2} P_{2}^{\prime}}\right)\left(\boldsymbol{\Omega}_{1, \tilde{b}^{\prime}}+\mathbf{\Omega}_{2, \tilde{b}^{\prime}}\right) \\
& -\sum_{j=1}^{\ell^{\prime}-1}\left(b_{1, \ell^{\prime}, j}+b_{2, \ell^{\prime}, j}\right) \overline{\mathbf{V}}_{2,(b-1) \eta+j} \\
& -a_{2, \ell^{\prime}} \mathbf{\Xi}_{2, b} \\
= & a_{1, \ell^{\prime}} \mathbf{\Xi}_{1, b} \\
& +\sum_{j=1}^{\ell^{\prime}-1} b_{1, \ell^{\prime}, j}\left(\mathbf{W}_{1,(b-1) \eta+j}-\mathbf{W}_{2,(b-1) \eta+j}\right) \\
& +\mathbf{Z}_{\tilde{b}^{\prime}}+\mathbf{W}_{2, \tilde{b}^{\prime}}
\end{aligned}
$$

where $\quad \mathbf{Z}_{\tilde{b}^{\prime}} \triangleq \quad\left(Z_{\left(\tilde{b}^{\prime}-1\right) n+1}, \ldots, Z_{\tilde{b}^{\prime} n}\right)^{\top}$. Transmitter 2 finally decodes Message $M_{1, \mathrm{CL}, \tilde{b}}$ based on $\tilde{\mathbf{N}}_{2,(b-1) \eta+1}, \ldots, \tilde{\mathbf{N}}_{2,(b-1) \eta+\ell-1}$, and $\tilde{\mathbf{V}}_{2,(b-1) \eta+\ell}$ using an optimal decoder for a single-input antenna/multi-output antenna Gaussian channel with correlated but temporally-white noise sequences.

We next describe the decoding at the receiver. We first consider the decoding of the pair $\left(M_{1, \mathrm{CL}, \tilde{b}}, M_{2, \mathrm{CL}, \tilde{b})}\right)$ after a fixed subblock $\tilde{b} \in\{\eta+1, \ldots,(B+1) \eta\}$. Define $b \in\{2, \ldots, B+1\}$ and $\ell \in\{1, \ldots, \eta\}$ so that $\tilde{b}=(b-1) \eta+\ell$. Before describing the decoding of the pair $\left(M_{1, \mathrm{CL} . \tilde{b}}, M_{2, \mathrm{CL} . \tilde{b}}\right)$ at the end of this paragraph, we notice the following. In decoding steps after previous subblocks the receiver has already decoded Messages $\quad\left\{\left(M_{1, \mathrm{ICL},(b-3) \eta+\ell^{\prime}}, M_{2, \mathrm{ICL},(b-3) \eta+\ell^{\prime}}\right)\right\}_{\ell^{\prime}=1}^{\ell}$, $\left\{\left(M_{1, \mathrm{ICL},(b-2) \eta+\ell^{\prime}}, M_{2, \mathrm{ICL},(b-2) \eta+\ell^{\prime}}\right)\right\}_{\ell^{\prime}=1}^{\ell}, \quad$ and

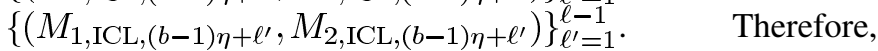
(assuming that these decodings were successful) the receiver can reconstruct the sequences produced to encode these messages and subtract them from the output signal. Thus, the receiver can compute for $b^{\prime} \in\{b-1, b\}$ and $\ell^{\prime} \in\{1, \ldots, \ell-1\}$ the "cleaned" output vector

$$
\begin{aligned}
\overline{\mathbf{Y}}_{\left(b^{\prime}-1\right) \eta+\ell^{\prime}} & \mathbf{Y}_{\left(b^{\prime}-1\right) \eta+\ell^{\prime}}-\sqrt{\left(1-\rho_{1}^{2}\right) P_{1}^{\prime}} \mathbf{U}_{1,\left(b^{\prime}-1\right) \eta+\ell^{\prime}} \\
- & \sqrt{\left(1-\rho_{2}^{2}\right) P_{2}^{\prime}} \mathbf{U}_{2,\left(b^{\prime}-1\right) \eta+\ell^{\prime}} \\
- & \left(\sqrt{\frac{1}{2} \rho_{1}^{2} P_{1}^{\prime}}+\sqrt{\frac{1}{2} \rho_{2}^{2} P_{2}^{\prime}}\right) \\
& \cdot\left(\boldsymbol{\Omega}_{1,\left(b^{\prime}-1\right) \eta+\ell^{\prime}}+\boldsymbol{\Omega}_{2,\left(b^{\prime}-1\right) \eta+\ell^{\prime}}\right)
\end{aligned}
$$

$$
\begin{aligned}
= & a_{1, \ell} \boldsymbol{\Xi}_{1, b^{\prime}}+a_{2, \ell^{\prime}} \mathbf{\Xi}_{2, b^{\prime}} \\
& +\sum_{j=1}^{\ell^{\prime}-1}\left(b_{1, \ell^{\prime}, j} \overline{\mathbf{V}}_{1,\left(b^{\prime}-1\right) \eta+j}+b_{2, \ell^{\prime}, j} \overline{\mathbf{V}}_{2,\left(b^{\prime}-1\right) \eta+j}\right) \\
& +\mathbf{Z}_{\left(b^{\prime}-1\right) \eta+\ell^{\prime}},
\end{aligned}
$$

and

$$
\mathbf{Y}_{\left(b^{\prime}-1\right) \eta+\ell^{\prime}} \triangleq\left(Y_{\left(\left(b^{\prime}-1\right) \eta+\ell^{\prime}-1\right) n+1}, \ldots, Y_{\left(\left(b^{\prime}-1\right) \eta+\ell^{\prime}\right) n}\right)^{\top}
$$

and it can compute

$$
\begin{aligned}
\overline{\mathbf{Y}}_{(b-2) \eta+\ell}^{(2)} & \mathbf{Y}_{(b-2) \eta+\ell} \\
& -\left(\sqrt{\frac{1}{2} \rho_{1}^{2} P_{1}^{\prime}}+\sqrt{\frac{1}{2} \rho_{2}^{2} P_{2}^{\prime}}\right) \\
& \cdot\left(\boldsymbol{\Omega}_{1,(b-2) \eta+\ell}+\boldsymbol{\Omega}_{2,(b-2) \eta+\ell}\right) \\
= & \sqrt{\left(1-\rho_{1}^{2}\right) P_{1}^{\prime}} \mathbf{U}_{1,(b-2) \eta+\ell} \\
& +\sqrt{\left(1-\rho_{2}^{2}\right) P_{2}^{\prime}} \mathbf{U}_{2,(b-2) \eta+\ell}+a_{1, \ell} \boldsymbol{\Xi}_{1, b}+a_{2, \ell} \mathbf{\Xi}_{2, b} \\
& +\sum_{j=1}^{\ell-1}\left(b_{1, \ell, j} \overline{\mathbf{V}}_{1,(b-2) \eta+j}+b_{2, \ell, j} \overline{\mathbf{V}}_{2,(b-2) \eta+j}\right) \\
& +\mathbf{Z}_{(b-2) \eta+\ell}
\end{aligned}
$$

Notice that the "cleaned" output vector $\overline{\mathbf{Y}}_{\left(b^{\prime}-1\right) \eta+\ell^{\prime}}$ equals the difference $\left(\overline{\mathbf{V}}_{1,\left(b^{\prime}-1\right) \eta+\ell^{\prime}}-\mathbf{W}_{1,\left(b^{\prime}-1\right) \eta+\ell^{\prime}}\right)$. Notice further, that even though the "cleaned" outputs $\quad \overline{\mathbf{Y}}_{(b-2) \eta+1}, \ldots, \overline{\mathbf{Y}}_{(b-2) \eta+\ell-1} \quad$ and $\overline{\mathbf{Y}}_{(b-1) \eta+1}, \ldots, \overline{\mathbf{Y}}_{(b-1) \eta+\ell-1}$ do not depend on the pair $\left(M_{1, \mathrm{CL}, \tilde{b}}, M_{2, \mathrm{CL}, \tilde{b}}\right)$, they are correlated with the noise sequences corrupting $\overline{\mathbf{Y}}_{(b-2) \eta+\ell}^{(2)}$ and $\mathbf{Y}_{(b-1) \eta+\ell}$ and should be taken into account by the receiver when decoding $\left(M_{1, \mathrm{CL}, \tilde{b}}, M_{2, \mathrm{CL}, \tilde{b}}\right)$. Thus, the receiver should decode the pair $\left(M_{1, \mathrm{CL}, \tilde{b}}, M_{2, \mathrm{CL}, \tilde{b}}\right)$ based on the vectors $\overline{\mathbf{Y}}_{(b-2) \eta+1}, \ldots, \overline{\mathbf{Y}}_{(b-2) \eta+\ell-1}, \mathbf{\mathbf { Y }}_{(b-1) \eta+1}, \ldots, \overline{\mathbf{Y}}_{(b-1) \eta+\ell-1}$, $\overline{\mathbf{Y}}_{(b-2) \eta+\ell}^{(2)}$, and $\overline{\mathbf{Y}}_{(b-1) \eta+\ell}$. To this end, the receiver first partly "decorrelates" the vectors by computing

$$
\begin{aligned}
\tilde{\mathbf{Y}}_{(b-1) \eta+\ell} & \mathbf{Y}_{(b-1) \eta+\ell}-\sum_{j=1}^{\ell-1}\left(b_{1, \ell, j}+b_{2, \ell, j}\right) \overline{\mathbf{Y}}_{(b-1) \eta+j} \\
= & \sqrt{\left(1-\rho_{1}^{2}\right) P_{1}^{\prime}} \mathbf{U}_{1,(b-1) \eta+\ell} \\
& +\sqrt{\left(1-\rho_{2}^{2}\right) P_{2}^{\prime}} \mathbf{U}_{2,(b-1) \eta+\ell} \\
& +\left(\sqrt{\frac{1}{2} \rho_{1}^{2} P_{1}^{\prime}}+\sqrt{\frac{1}{2} \rho_{2}^{2} P_{2}^{\prime}}\right) \\
& \cdot\left(\boldsymbol{\Omega}_{1,(b-1) \eta+\ell}+\boldsymbol{\Omega}_{2,(b-1) \eta+\ell}\right) \\
& +a_{1, \ell} \boldsymbol{\Xi}_{1, b}+a_{2, \ell} \boldsymbol{\Xi}_{2, b} \\
& \quad \sum_{j-1}\left(b_{1, \ell, j} \mathbf{W}_{1,(b-1) \eta+j}+b_{2, \ell, j} \mathbf{W}_{2,(b-1) \eta+j}\right) \\
& +\mathbf{Z}_{(b-1) \eta+\ell}, \\
\tilde{\mathbf{Y}}_{(b-2) \eta+\ell}^{(2)} &
\end{aligned}
$$




$$
\begin{aligned}
\triangleq & \overline{\mathbf{Y}}_{(b-2) \eta+\ell}^{(2)}-\sum_{j=1}^{\ell-1}\left(b_{1, \ell, j}+b_{2, \ell, j}\right) \overline{\mathbf{Y}}_{(b-2) \eta+j} \\
= & \sqrt{\left(1-\rho_{1}^{2}\right) P_{1}^{\prime}} \mathbf{U}_{1,(b-2) \eta+\ell} \\
& +\sqrt{\left(1-\rho_{2}^{2}\right) P_{2}^{\prime}} \mathbf{U}_{2,(b-2) \eta+\ell} \\
& +a_{1, \ell} \Xi_{1, b}+a_{2, \ell} \Xi_{2, b} \\
& +\sum_{j=1}^{\ell-1}\left(b_{1, \ell, j} \overline{\mathbf{W}}_{1,(b-2) \eta+j}+b_{2, \ell, j} \overline{\mathbf{W}}_{2,(b-2) \eta+j}\right) \\
& +\mathbf{Z}_{(b-2) \eta+\ell}
\end{aligned}
$$

and for $b^{\prime} \in\{b-1, b\}, \ell^{\prime} \in\{1, \ldots, \ell-1\}$ :

$$
\begin{aligned}
& \tilde{\mathbf{Y}}_{\left(b^{\prime}-1\right) \eta+\ell^{\prime}}^{(3)} \\
& \triangleq \overline{\mathbf{Y}}_{\left(b^{\prime}-1\right) \eta+\ell^{\prime}} \\
& \quad-\sum_{j=1}^{\ell^{\prime}-1}\left(b_{1, \ell^{\prime}, j}+b_{2, \ell^{\prime}, j}\right) \overline{\mathbf{Y}}_{\left(b^{\prime}-1\right) \eta+j} \\
& =a_{1, \ell^{\prime}} \boldsymbol{\Xi}_{1, b^{\prime}}+a_{2, \ell^{\prime}} \boldsymbol{\Xi}_{2, b^{\prime}} \\
& \quad+\sum_{j=1}^{\ell^{\prime}-1}\left(b_{1, \ell^{\prime}, j} \mathbf{W}_{1,\left(b^{\prime}-1\right) \eta+j}+b_{2, \ell^{\prime}, j} \mathbf{W}_{2,\left(b^{\prime}-1\right) \eta+j}\right) \\
& \quad+\mathbf{Z}_{\left(b^{\prime}-1\right) \eta+\ell^{\prime}} .
\end{aligned}
$$

The receiver then decodes the pair of messages $\quad\left(M_{1, \text { ICL }, \tilde{b}}, M_{2, \text { ICL }, \tilde{b}}\right) \quad$ based $\quad$ on $\tilde{\mathbf{Y}}_{(b-2) \eta+1}^{(3)}, \ldots, \tilde{\mathbf{Y}}_{(b-2) \eta+\ell-1}^{(3)}, \tilde{\mathbf{Y}}_{(b-2) \eta+\ell}^{(2)}$ and $\tilde{\mathbf{Y}}_{(b-1) \eta+1}^{(3)}, \ldots, \tilde{\mathbf{Y}}_{(b-1) \eta+\ell-1}^{(3)}, \tilde{\mathbf{Y}}_{(b-1) \eta+\ell}$ using an optimal decoder for a 2 -input/2 $\ell$-output antenna Gaussian MAC with temporally-white noise sequences correlated across antennas.

After decoding Messages $\left\{\left(M_{1, \mathrm{ICL}, \tilde{b}}, M_{2, \mathrm{ICL}, \tilde{b}}\right)\right\}_{\tilde{b}=1}^{B \eta}$ the receiver decodes Messages $\left\{\left(M_{1, \mathrm{ICS}, b}, M_{2, \mathrm{ICS}, b}\right)\right\}_{b=1}^{B}$. To this end, it first reverses the interleaving introduced by the modified inner encoders on the "cleaned" output vectors $\overline{\mathbf{Y}}_{1}, \ldots, \overline{\mathbf{Y}}_{B \eta}$. That is, for $b \in\{1, \ldots, B\}$, it constructs the $\eta n$-dimensional vector

$$
\begin{aligned}
\mathbf{Y}_{\text {DeInt }, b} \triangleq & \left(\bar{Y}_{(b-1) \eta+1,1}, \ldots, \bar{Y}_{b \eta, 1},\right. \\
& \bar{Y}_{(b-1) \eta+1,2}, \ldots, \bar{Y}_{b \eta, 2}, \\
& \left.\bar{Y}_{(b-1) \eta+1, n}, \ldots, \bar{Y}_{b, \eta n}\right)^{\top}
\end{aligned}
$$

where $\bar{Y}_{\tilde{b}, i}$ denotes the $i$ th entry of vector $\overline{\mathbf{Y}}_{\tilde{b}}$. It then decodes Messages $\left(M_{1, \mathrm{ICS}, b}, M_{2, \mathrm{ICS}, b}\right)$ applying inner and outer decoder of the concatenated scheme to the vector $\mathbf{Y}_{\text {DeInt }, b}$.

\section{A. Noisy and Perfect Partial Feedback}

The proposed extension can also be applied in settings with noisy or perfect partial feedback, if $B_{1}$ is set to the all-zero matrix and if Carleial's scheme for noisy or perfect partial feedback is applied. Accordingly, our scheme should be modified so that there is no decoding taking place at Transmitter 1 . Therefore, in (210) and (211), the term $\sqrt{\frac{1}{2} \rho_{\nu}^{2} P_{\nu}^{\prime}}\left(\boldsymbol{\omega}_{1, \tilde{b}}+\boldsymbol{\omega}_{2, \tilde{b}}\right)$ should be replaced by $\sqrt{\rho_{\nu}^{2} P_{\nu}^{\prime}} \boldsymbol{\omega}_{1, \tilde{b}}$, for $\nu \in\{1,2\}$.

Notice that-as in the second extension-for perfect partial feedback the various vectors computed for the decodings at
Transmitter 2 and for the decodings at the receiver have uncorrelated noise components. Therefore, without loss in optimality, Transmitter 2 and the receiver can use optimal decoders for Gaussian multi-input antenna/multi-output antenna channels with independent white noise sequences.

\section{ACKNOWLEDGMENT}

The authors acknowledge helpful discussions with M. Gastpar and G. Kramer.

\section{REFERENCES}

[1] S. I. Bross, A. Lapidoth, and M. A. Wigger, "The Gaussian MAC with conferencing encoders," in Proc. IEEE Int. Symp. Inf. Theory, Toronto, ON, Canada, Jul. 6-11, 2008.

[2] A. B. Carleial, "Multiple-access channels with different generalized feedback signals," IEEE Trans. Inf. Theory, vol. IT-28, pp. 841-850, Nov. 1982.

[3] T. M. Cover, "Some advances in broadcast channels," in Advances in Communication Systems, A. Viterbi, Ed. San Francisco, CA: Academic, 1975, vol. 4.

[4] T. M. Cover and C. S. K. Leung, "An achievable rate region for the multiple-access channel with feedback," IEEE Trans. Inf. Theory, vol. IT-27, pp. 292-298, May 1981.

[5] N. T. Gaarder and J. K. Wolf, "The capacity region of a multiple-access discrete memoryless channel can increase with feedback," IEEE Trans. Inf. Theory, vol. IT-21, pp. 100-102, Jan. 1975.

[6] M. Gastpar, "On noisy feedback in Gaussian networks," in Proc. 43rd Allerton Conf. Comm., Contr. and Comp., Monticello, IL, 2005.

[7] M. Gastpar and G. Kramer, "On cooperation via noisy feedback," in Proc. IZS, Feb. 22-24, 2006, pp. 146-149.

[8] A. P. Hekstra and F. M. J. Willems, "Dependence balance bounds for single-output two-way channels," IEEE Trans. Inf. Theory, vol. 35, pp. 44-53, Jan. 1989.

[9] Y.-H. Kim, A. Lapidoth, and T. Weissman, "Bounds on the error exponent of the AWGN channel with AWGN-corrupted feedback," in Proc. 24th IEEE Conv. Electrical \& Electronics Eng in Israel (IEEEI'06), Eilat, Israel, Nov. 15-17, 2006, pp. 184-188.

[10] G. Kramer, "Directed Information for Channels With Feedback," Ph.D. thesis, ETH Zurich, Zurich, Switzerland, 1998.

[11] G. Kramer, "Feedback strategies for white Gaussian interference networks," IEEE Trans. Inf. Theory, vol. 48, pp. 1423-1438, Jun. 2002.

[12] G. Kramer, "Capacity results for the discrete memoryless network," IEEE Trans. Inf. Theory, vol. 49, pp. 4-21, Jan. 2003.

[13] L. H. Ozarow, "The capacity of the white Gaussian multiple-access channel with feedback," IEEE Trans. Inf. Theory, vol. IT-30, pp. 623-629, Jul. 1984.

[14] B. Rimoldi and R. Urbanke, "A rate-splitting approach to the Gaussian multiple-access channel," IEEE Trans. Inf. Theory, vol. 42, pp. 364-375, 1996.

[15] J. P. M. Schalkwijk and T. Kailath, "A coding scheme for additive noise channels with feedback-I:No bandwidth constraint," IEEE Trans. Inf. Theory, vol. IT-12, pp. 172-182, Apr. 1966.

[16] D. Shaviv and Y. Steinberg, "On the multiple access channel with common rate-limited feedback," in Proc. IZS, Mar. 12-14, 2008, pp. $108-111$.

[17] R. Tandon and S. Ulukus, "Dependence balance based outer bounds for Gaussian networks with cooperation and feedback," IEEE Trans. Inf. Theory, submitted for publication.

[18] E. C. van der Meulen, "Capacity theorems for multiple-access channels with feedback," in ISICT'87, Campinas, Brazil, Jul. 1, 1987.

[19] V. Venkatesan, "Optimality of Gaussian inputs for a multi-access achievable region," in Semester Project, Signal and Inform. Proc. Lab., ETH Zurich, Switzerland, Jun. 2007.

[20] F. M. J. Willems, "The feedback capacity region of a class of discrete memoryless multiple-access channels," IEEE Trans. Inf. Theory, vol. IT-28, pp. 93-95, Jan. 1982.

[21] F. M. J. Willems and E. C. van der Meulen, "Partial feedback for the discrete memoryless multiple access channel," IEEE Trans. Inf. Theory, vol. IT-29, pp. 287-290, Mar. 1983.

[22] F. M. J. Willems, E. C. van der Meulen, and J. P. M. Schalkwijk, "A coding scheme for the additive white Gaussian noise multiple access channel with semi-feedback," Tijdschrift van het Nederlands Elektronica-en Radiogenootschap, vol. 48, no. 3, pp. 103-107, 1983. 
[23] F. M. J. Willems, E. C. van der Meulen, and J. P. M. Schalkwijk, "Generalized feedback for the discrete memoryless multiple access channel," in Proc. 21th Allerton Conf. Comm., Contr. and Comp., Monticello, IL, Oct. 5-7, 1983, pp. 284-292.

[24] J. M. Wozencraft and I. M. Jacobs, Principles of Communication Engineering. New York: Wiley, 1965.

[25] A. D. Wyner, "Recent results in the Shannon theory," IEEE Trans. Inf. Theory, vol. IT-20, pp. 2-10, Jan. 1974.

Amos Lapidoth (S'89-M'95-SM'00-F'04) received the B.A. degree in mathematics (summa cum laude) in 1986, the B.Sc. degree in electrical engineering (summa cum laude) in 1986, and the M.Sc. degree in electrical engineering in 1990, all from The Technion-Israel Institute of Technology, Haifa. He received the Ph.D. degree in electrical engineering from Stanford University, Stanford, CA, in 1995.

From 1995 to 1999, he was an Assistant and Associate Professor with the Department of Electrical Engineering and Computer Science at the Massachusetts
Institute of Technology, Cambridge, and was the KDD Career Development Associate Professor in Communications and Technology. He is now Professor of Information Theory at ETH Zurich, Switzerland. He is the author of the book A Foundation in Digital Communication (Cambridge University Press, 2009). His research interests are in digital communications and information theory.

Dr. Lapidoth served as Associate Editor for Shannon Theory for the IEEE TRANSACTIONS ON INFORMATION THEORY in the years 2003-2004 and 2009.

Michèle Wigger (S'05-M'09) received the M.Sc. degree in electrical engineering (with distinction) and the Ph.D. degree in electrical engineering both from ETH Zurich, Switzerland, in 2003 and 2008, respectively.

In 2009, she was a postdoctoral researcher at the ITA center at the University of California, San Diego. Since December 2009, she has been an Assistant Professor at Telecom ParisTech, Paris, France. Her research interests are in information and communications theory; in particular in wireless networks, feedback channels, and channels with states. 